\title{
Polyhedral clustering
}

Citation for published version (APA):

Rutten, J. H. G. C. (1998). Polyhedral clustering. [Doctoral Thesis, Maastricht University]. Universiteit Maastricht. https://doi.org/10.26481/dis.19980604jr

Document status and date:

Published: 01/01/1998

DOI:

10.26481/dis.19980604jr

Document Version:

Publisher's PDF, also known as Version of record

\section{Please check the document version of this publication:}

- A submitted manuscript is the version of the article upon submission and before peer-review. There can be important differences between the submitted version and the official published version of record.

People interested in the research are advised to contact the author for the final version of the publication, or visit the DOI to the publisher's website.

- The final author version and the galley proof are versions of the publication after peer review.

- The final published version features the final layout of the paper including the volume, issue and page numbers.

Link to publication

\footnotetext{
General rights rights.

- You may freely distribute the URL identifying the publication in the public portal. please follow below link for the End User Agreement:

www.umlib.nl/taverne-license

Take down policy

If you believe that this document breaches copyright please contact us at:

repository@maastrichtuniversity.nl

providing details and we will investigate your claim.
}

Copyright and moral rights for the publications made accessible in the public portal are retained by the authors and/or other copyright owners and it is a condition of accessing publications that users recognise and abide by the legal requirements associated with these

- Users may download and print one copy of any publication from the public portal for the purpose of private study or research.

- You may not further distribute the material or use it for any profit-making activity or commercial gain

If the publication is distributed under the terms of Article $25 \mathrm{fa}$ of the Dutch Copyright Act, indicated by the "Taverne" license above, 


\title{
Polyhedral clustering
}

\author{
Proefschrift
}

ter verkrijging van de graad van doctor aan de Universiteit Maastricht, op gezag van de Rector Magnificus, prof.dr. A.C. Nieuwenhuijzen Kruseman, volgens het besluit van het College van Decanen, in het openbaar te verdedigen op donderdag 4 juni 1998 om 12.00 uur

door

Jeroen Hendrik Gerardus Christiaan Rutten 


\section{Promotor:}

Prof.dr.ir.drs. O.J. Vrieze

\section{Copromotor:}

Dr. F.C.R. Spieksma

\section{Beoordelingscommissie:}

Prof.dr. H. Peters (voorzitier)

Prof.dr. H.J. Bandelt (Universiteit Hamburg)

Prof.dr. U. Faigle (Universiteit Twente)

Dr.ir. C.P.M. van Hoesel

Prof.dr. L.A. Wolsey (Université Catholique de Louvain)

\section{$\star$ \\ UPM}

UNIERSITAIRE PERS MAASTRICHT

Polyhedral clustering

(c) J.H.G.C. Rutten 1998

Proefschrift Universiteit Maastricht.

Samenvatting in het; Nederlands en in het Engels.

ISBN 9052782369

Trefwoorden: polyhedral combinatorics, clustering, graph partitioning, patching, facets, complexity. 


\section{Preface}

During my Ph.D. research I have been fortunate to be surrounded by many people who supported me and provided me with critical but helpful comments, thus contributing one way or the other to this thesis. First of all, there is the contribution of Koos Vrieze. He created an open research atmosphere and offered me full scope to develop my talents. Next comes the contribution of Hans-Jürgen Bandelt, Maarten Oosten, and Frits Spieksma as co-authors of articles on which some of the chapters are based. The contribution of Janos Flesch is less visible, but not less important. We worked together on several courses on operations research, and we had many fruitful discussions on all kinds of subjects, including but not restricted to mathematics. Janos, thanks for all the things we have done together and for being a friend.

At least two people contributed to this thesis by their moral support: Hélène Geldof and Stan van Hoesel. Several times during the past four years I felt like I was walking in a valley: surrounded by a fascinating environment, but lacking a good perspective. Hélène always tried to push me forward, but if she did not succeed she would say: "Have a word with Stan." Stan usually succeeded in revealing new tracks leading up hi]l, and I always left his room in a better mood than I came in. Hélène and Stan, your support meant, more to me than you will probably realize.

Furthermore, I thank Susan Geldof for the very careful proofreading of an earlier version of this thesis. Finally, I thank my parents for the opportunities they gave me. Without them this thesis would never have existed in the first place.

Jeroen Rutten 


\section{Contents}

1 Clustering 1

1.1 As ubiquitous as clustering . . . . . . . . . . . . . 1

1.2 Approaches to clustering . . . . . . . . . . . . 6

1.3 Polyhedral theory . . . . . . . . . . . . . . . . . . . . 8

1.4 Graph theory . . . . . . . . . . . . . . . . 12

1.5 Related polyhedra . . . . . . . . . . . . . . 14

1.6 How to read this book ................. 18

2 Clique partitioning $\quad 21$

2.1 Introduction . . . . . . . . . . . . . . . 21

2.2 Zero lifting . . . . . . . . . . . . . . . . . . . . . . . . . . . . . . . .

2.3 Recognizing facets . . . . . . . . . . . . . . . . . . . . . . . . . .

2.4 General 2-partition inequalities ............ 34

3 New facets and patching $\quad 39$

3.1 Introduction . . . . . . . . . . . . . . . . . . . 39

3.2 New facets. . . . . . . . . . . . . . . . . 42

3.3 Lifting . . . . . . . . . . . . . . . . 58

3.4 Patching . . . . . . . . . . . . . . . . 61

3.5 Facets with right-hand side 1 or $2 \ldots \ldots 76$

4 Small polytopes $\quad \mathbf{8 3}$

4.1 Introduction. . . . . . . . . . . . . . . . 83

4.2 Complete and partial descriptions . . . . . . . . . 84

4.3 New facets . . . . . . . . . . . . . . . . . 85 
5 Strength $\quad 93$

5.1 Introduction . . . . . . . . . . . . . . . . . . . . . . .

5.2 Measures for strength . . . . . . . . . . . . . . . . . . 94

5.3 The strength of small facets . . . . . . . . . . . . . . 98

5.4 The strength of facets of $P\left(K_{n}\right) \ldots \ldots \ldots 9$

6 Separation 111

6.1 Introduction . . . . . . . . . . . . . . . 111

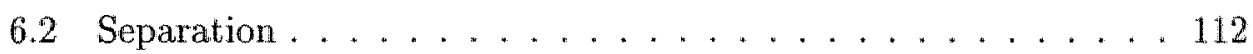

7 Disconnecting graphs $\quad \mathbf{1 2 5}$

7.1 Introduction. . . . . . . . . . . . . . 125

7.2 Two formulations . . . . . . . . . . . . . . . 127

7.3 Polyhedral results. . . . . . . . . . . . . . . . . 130

7.4 Computational results . . . . . . . . . . . . . . . 144

7.5 Conclusions . . . . . . . . . . . . . 155

8 Patching $\quad 157$

8.1 Introduction . . . . . . . . . . . . . 157

8.2 Intersection patching . . . . . . . . . . . . 158

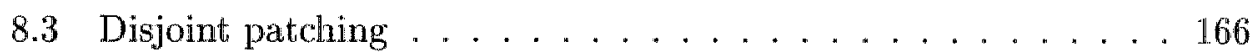

$\begin{array}{ll}\text { Bibliography } & 169\end{array}$

$\begin{array}{ll}\text { Summary } & 175\end{array}$

$\begin{array}{ll}\text { Samenvatting } & 177\end{array}$

$\begin{array}{ll}\text { Curriculum vitae } & 179\end{array}$

$\begin{array}{ll}\text { Index } & 181\end{array}$ 


\section{Chapter 1}

\section{Clustering}

\subsection{As ubiquitous as clustering}

Clustering is among the most widely spread phenomena in the universe. In previous centuries scientists already noticed that the stars are not uniformly spread through the universe, but instead form galaxies. And even these galaxies are not randomly distributed in space. In the 1980's astronomers discovered that they form clusters (e.g. the "Virgo cluster"), or even super-clusters (e.g. the "Big Wall"), and that the space between these clusters is relatively empty. On a smaller scale we also find evidence that clustering is one of the most ubiquitous phenomena encountered. Galaxies are made up of groups of stars, and many stars form the center of a solar system, just like the one the earth belongs to. If we focus on the earth we see that land and water form clusters, with names like "Europe" and "Pacific Ocean". And within these clusters we find smaller clusters, and within these smaller clusters we find tiny clusters, and so on, until we arrive at a single atom. A single atom? Well, actually it is a cluster of protons and neutrons, surrounded by electrons.

What causes clustering to be so ubiquitous? There is a number of reasons for this. First of all, a physical one. Atoms, for instance in a piece of gold, form a crystal structure in such a way that the total amount of energy is minimized. This also holds on a larger scale: due to gravity the galaxies form clusters so as to minimize the potential energy (this is not contradictory to the "Big. Bang" theory: some galaxies move so fast away from each other that they 
will never form a cluster, despite the gravity). Next, there are economical reasons for clustering. In the financial world it is very common that banks and insurance companies locate their offices close to each other, or close to the stock exchange. This allows them to react quickly on changes in the market, and it makes cooperation easier. Industrial companies benefit from short transportation lines. The products of one firm are the raw materials for the other, and a third firm makes the machines to produce consumer products from these raw materials. Even in telecommunications and computer industry, where distance seems to be of minor importance, companies benefit from being together. For hardware and software industry Silicon Valley is the place to be.

Third, there are biological reasons for clustering. Lions, for instance, chase gnus and zebras for food, and a golden eagle chases, among other animals, rabbits and mice. In general, a predator will have to live in the same habitat as its pray. Clustering in nature is not solely caused by a predator-pray relationship, but sometimes it is beneficial for both parties to except each others presence. Buffaloes benefit from birds that pick all kinds of insects out of their fur, and for these birds it is an easy way to make a living. Gnus form herds to protect themselves from lions and other predators. Even plant and animals sometimes have mutual advantage from each others vicinity. Squirrels profit from oaks, because the trees form a place to hide and they provide the squirrels with food. But at the same time the oaks profit from the squirrels, since they help to spread the acorns. A very obvious reason for clustering in nature is to avoid the extinction of species: a male and female animal simply have to meet, otherwise there will be no posterity.

Finally, there are numerous practical reasons for clustering. The books in a university library are put on the shelves according to subject, just to simplify the retrieval of information. Books in a public library are usually grouped by author. Spare parts in a ware house may be stored according to demand, or according to other storage requirements, such as the dimensions. Some photographic chemicals need to be stored in a refrigerated environment, whereas the unprocessed films have to be stored in absolute darkness. In a supermarket different brands of the same product are put next to each other, to make comparison easier. One could say that clustering creates order in chaos, and hidden structures become visible. This is probably one of the reasons why so much research has been done on clustering problems.

Let me give a very general formulation of the kind of clustering problems considered in this thesis. Given is a set of objects, and each object has a 
number of characteristics. The problem is to partition the set of objects into a number of subsets, such that objects in the same subset resemble one another, and objects in different subsets are completely unlike. This might look like an easy problem at first sight, but it turns out to be a very hard problem in general. What should one do if object 1 and 2 resemble each other, as well as object 2 and 3, and object 3 and 4, but object 1 and 4 are very different? Must we put objects 1 thru 4 in the same subset, and allowing two unlike objects to be in the same cluster, or must we put them in two or more subsets, thereby separating some resembling objects? This dilemma can be solved by giving an exact formulation of 'resemblance' and optimizing an objective which is a function of the partitioning.

Consider for instance a biologist studying all kinds of wild cats. After spending several months in the Savannah of Africa, doing almost nothing but research, he has encountered 7 different species, and he has investigated 7 different characteristics, as shown in Table 1.1 (this table is part of a real world problem found in [38]). The characteristics concern: markings of the pelt, the fur, the ears, the height, weight and length of the body, and the length of the tail compared to the length of the body.

\begin{tabular}{|ll|ccccccc|}
\hline & Wild cats & 1 & 2 & 3 & 4 & 5 & 6 & 7 \\
\hline \hline 1 & Lion & 1 & 0 & 1 & 3 & 3 & 3 & 2 \\
2 & Tiger & 3 & 0 & 1 & 3 & 3 & 3 & 2 \\
3 & Jaguar & 2 & 0 & 1 & 3 & 3 & 2 & 1 \\
4 & Leopard & 2 & 0 & 1 & 3 & 3 & 2 & 2 \\
5 & Once & 2 & 1 & 1 & 2 & 2 & 2 & 3 \\
6 & Guepard & 2 & 0 & 1 & 3 & 2 & 2 & 3 \\
7 & Puma & 1 & 0 & 1 & 2 & 3 & 2 & 3 \\
\hline
\end{tabular}

Table 1.1: Data matrix for the classification of wild cats.

There are several ways to define 'resemblance', and one of them is counting the number of common characteristics, and subtracting the number of characteristics in which two cats differ. The lion and the jaguar for instance, are equal with respect to four characteristics, but they differ with respect to the remaining three. As there is more than one way to define resemblance, there is also more than one possible objective (see the next section). When maximizing the total resemblance of all pairs of animals within the same group the optimal partitioning of these cats consists of two groups: one group consists 
of just the once, while the other group contains the remaining cats.

Another example of clustering is found in industry. A flexible manufacturing system is a complex of computer controlled machines, each of which is equipped with several tools, and each machine has to perform some tasks on a number of parts. An example is given in Table 1.2. There are four machines and four parts, and machine 1 has to process parts 1, 2, and 4, whereas machine 3 only has to process part 2 .

\begin{tabular}{|l|llll|}
\hline Parts $\rightarrow$ & 1 & 2 & 3 & 4 \\
\hline \hline Machine 1 & 1 & 1 & 0 & 1 \\
Machine 2 & 1 & 1 & 1 & 0 \\
Machine 3 & 0 & 1 & 0 & 0 \\
Machine 4 & 1 & 0 & 0 & 0 \\
\hline
\end{tabular}

Table 1.2: A machine-part matrix.

Due to resource constraints, e.g. time constraints or limited production area, the set of parts and machines has to be split into several groups, usually called (production) cells. If a part and a machine are assigned to the same cell, but this part does not have to be operated on by this machine, it is called a void. If a part and a machine are assigned to different cells, but this machine has to perform some task on this part, it is called an exception. Both voids and exceptions are undesirable, because voids lead to machine idle time, which is a waste of production capacity, and exceptions require the transportation of a part between different cells, which is in general a time consuming operation. The objective is to partition the set of parts and machines into a number of cells, such as to minimize the number of voids and exceptions. For the example of Table 1.2 the minimum number of voids and exceptions is 4 , and there exist several alternative cell formations that achieve this minimum.

VLSI design also gives rise to clustering problems. Modern micro processors typically consist of several million transistors, which are connected to each other through very tiny copper tracks. The transistors used nowadays are so fast that the length of the wiring is a limiting factor on the number of switchings per time unit, simply because the electric current cannot travel any faster than the speed of light. The problem is to place the transistors on the micro processor such that the total wiring length is kept within reasonable limits. If we define the resemblance of two connected transistors as 1, and the resem- 
blance of two unconnected transistors as -1 , we are left with the same kind of clustering problem as in the case of the classification of wild cats. Similar problems are found in compiler design and computer network architecture. Small computer networks often have a ring structure, and all computers in such a network can exchange data via this ring. In larger computer networks this structure quickly leads to network capacity problems. Instead of attaching all computers and file servers to a ring, this set of machines is split into a number of groups. All machines within the same group are connected to each other by a local area network (LAN, which might have a ring structure, but a star structure is also very common), and the LANs are connected via the so-called backbone. The LANs and the backbone together are called a wide area network (WAN). The only purpose of the backbone is to transport information between computers and file servers in different $\mathrm{I} A \mathrm{ANs}$. "The problem is to find an assignment of machines to LANs, such as to minimize (or reduce to a given levell) the data traffic load on the backbone.

A last example of clustering originates from pattern recognition (computer vision). During the Gulf war military satellites were used to observe Iraqi force movements, military buildings, and ground targets. The satellite images were processed by computers to identify tanks, planes, and all kinds of other objects. In order to do so the pixels of the image are compared to the surrounding pixels with respect to color, luminescence, and distance. Similar pixels are likely to belong to the same object (possibly background), whereas contrasting pixels describe contours. Identifying clusters of similar pixels is equivalent to identifying objects in the image.

As can be seen from the examples above clustering has a tremendous wealth of applications. The list could easily be extended with clozens of other examples found in different fields of biology, economy, or industry. However, we do not focus on the applications of clustering, but on methods for solving these problems. The remaining sections of this chapter are organized as follows. Section 1.2 briefly describes the general structure of most clustering problems, together with different approaches to these problems. One of these approaches uses polyhedral combinatorics, which is the subject of Section 1.3 and 1.5 . Section 1.3 mentions the most basic polyhedral definitions and results, and Section 1.5 gives an overview of some polyhedral problems that are related to clustering. Section 1.4 gives some definitions from graph theory, and we introduce most of the notation used throughout this thesis. The last section of this chapter gives an overview of the thesis. 


\subsection{Approaches to clustering}

Although clustering and classification are often used as synonyms classification is more. It contains clustering, but it also contains hierarchical structuring. Classification is hierarchical if it is nested, like the conceptual library classifications, or taxonomy in biology: the set of animals is split into the subset of vertebrates and the subset of invertebrates. The subset of vertebrates is subdivided further into e.g. mammals, birds, fishes, and reptiles. Subdivision goes on until the level of individual species. Consider, as another example, the mathematical problem of finding the roots of the quadratic equation $x^{2}+p x+q=0$. If $p^{2} \geq 4 q$ then the equation has two real roots; if $p^{2}<4 q$ both roots are complex. Figure 1.1 gives a complete hierarchical classification of the number of real and complex roots, and the signs of the real roots.

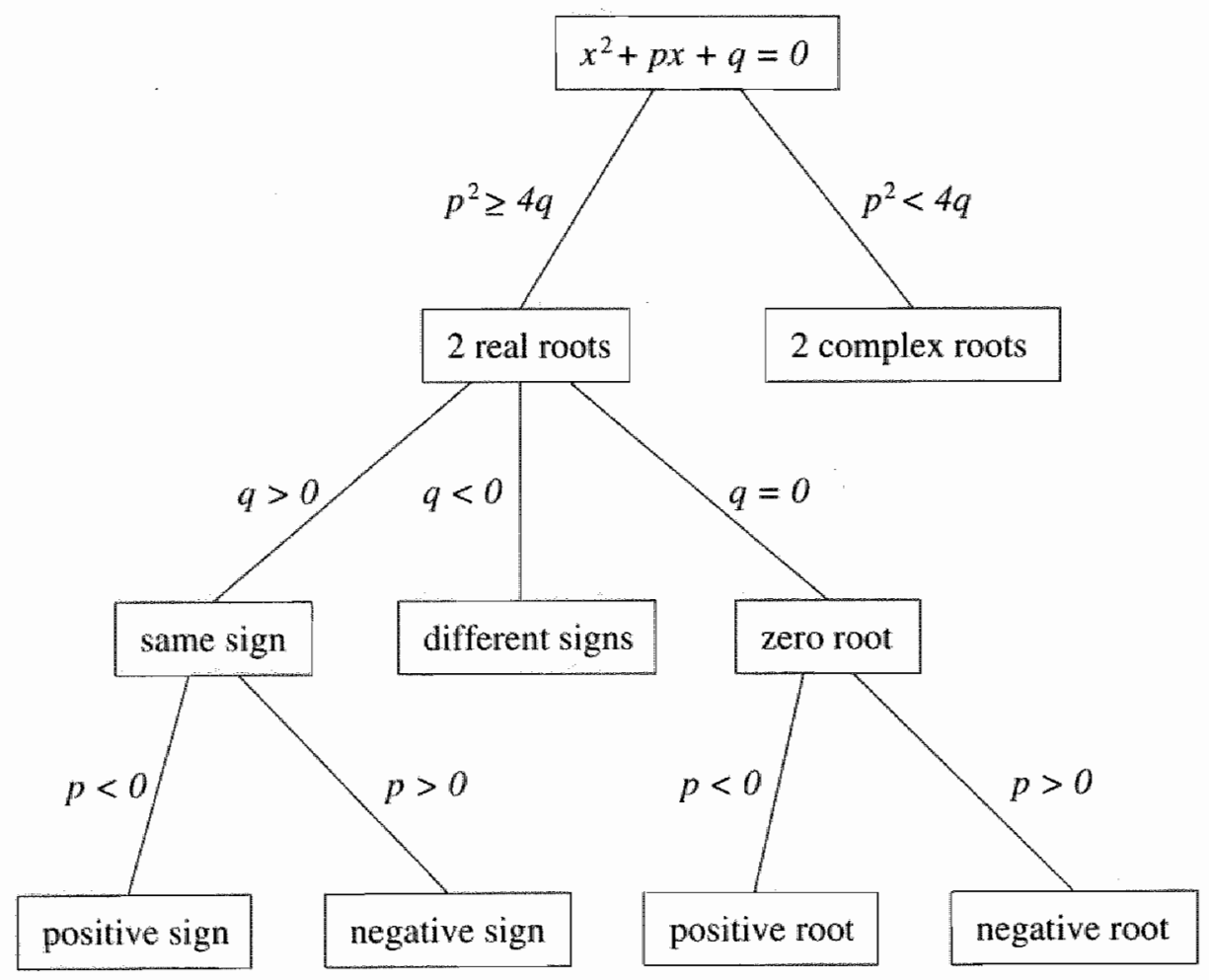

Figure 1.1: Hierarchical classification of the roots of a quadratic equation [54]. 
A very detailed treatment of classification is given in the book of Hartigan [44], and in the more recent work of Mirkin [54]. Here we consider the special case of clustering. The ingredients of most clustering problems are as follows.

- A set $N=\{1, \ldots, n\}$ of objects among which clusters are to be found.

- An $n \times p$ data matrix $M=\left(m_{i j}\right)$, where matrix entry $m_{i j}$ describes object $i \in N$ with respect to property $j \in\{1, \ldots, p\}$.

- Constraints on the clustering, e.g. on the minimum or maximum size of the clusters, or on the number of clusters.

- An objective.

A very well known example of clustering is the Periodic Chart, which arranges all elements according to some chemical and physical properties, varying from the number of protons in the kernel to the valency, i.e. the ability to interact with other elements. There are no restrictions on the clustering, and the objective is to find groups of elements that have similar chemical and physical properties. One solution to this clustering problem is to identify the columns of the Periodic Chart with clusters. In this way, elements within a cluster all have the same valency.

In particular, by comparing all pairs of (distinct) objects $i, j \in N$ we obtain two $n \times n$ matrices $D=\left(d_{i j}\right)$ and $S=\left(s_{i j}\right)$, where $d_{i j}$ is the number of dissimilarities between $i$ and $j$, and $s_{i j}$ is the number of similarities. A common objective is to find a partition $\left\{N_{1}, \ldots, N_{k}\right\}$ of $N$ ( $k$ is not fixed) that maximizes the minimum dissimilarity between objects in different subsets, or that minimizes the maximum dissimilarity between objects in the same subset. Another objective is to find a partition that maximizes the sum of dissimilarities between objects in different subsets, while the number of subsets is bounded from above. An overview of this kind of clustering problems is given by Hansen and Jaumard [43]. Related clustering problems arise when considering the similarity between objects. When taking into account both similarities and dissimilarities one could define $W:=S-D$, and ask for a partition $\left\{N_{1}, \ldots, N_{k}\right\}$ of $N$ that maximizes

$$
\sum_{l=1}^{k} \sum_{i, j \in N_{l}} w_{i j}
$$


with or without additional constraints on the partition. In case there are no additional constraints this problem is called the clique partitioning problem. Clique partitioning is known to be NP-hard [64], which implies that there are basically only two different approaches to it: an exact approach, that finds a best partition of the set of objects, even if it takes exponential time in the worst case, and a heuristic approach, which hopefully finds a good (but not necessarily best) partition within polynomial time. A mixture of these approaches is found in simulated annealing: this is a heuristic algorithm, but it finds an optimal partition with probability 1 if it is allowed to run arbitrarily long. Since our goal is to find optimal partitions in finite time, we adopt the exact polyhedral approach.

It should be pointed out here that the name 'clique partitioning' is sometimes also used for the problem of partitioning the vertex set of a graph into a minimal number of subsets, such that each subset induces a clique in the graph, i.e. finding a minimal clique cover [7].

\subsection{Polyhedral theory}

Most mathematical programming problems have their set of feasible solutions described implicitly, for instance the set of integer points $x \in \mathbb{Z}^{n}$ satisfying $A x \leq b$ for some real $m \times n$ matrix $A$ and some $b \in \mathbb{R}^{m}$, or the set of binary vectors corresponding to independent sets or Hamiltonian cycles in a graph. Polyhedral theory is the art of finding an explicit description of this set. This section briefly describes some basic concepts of polyhedral theory, the next section gives some definitions from graph theory and most of the notation used in the remaining chapters. The reader not familiar with polyhedral combinatorics is referred to Nemhauser and Wolsey [58] or Schrijver [63] for a very thorough introduction.

Definition 1.1 Given a set $S \subseteq \mathbb{R}^{n}$, a point $x \in \mathbb{R}^{n}$ is a convex combination of points in $S$ if there exists a finite set $\left\{s_{1}, \ldots, s_{k}\right\} \subseteq S$ and $\lambda \in \mathbb{R}_{+}^{k}$ with $\lambda_{\mathbb{1}}+\ldots+\lambda_{k}=\mathbb{1}$, such that $x=\lambda_{1} s_{1}+\ldots+\lambda_{k} s_{k}$. The convex hull of $S$, denoted by $\operatorname{conv}(S)$, is the set of all points that are convex combinations of points in $S$. The set $S$ is convex if $s_{1}, s_{2} \in S$ implies that $\lambda s_{1}+(1-\lambda) s_{2} \in S$ for all $0 \leq \lambda \leq 1$ 


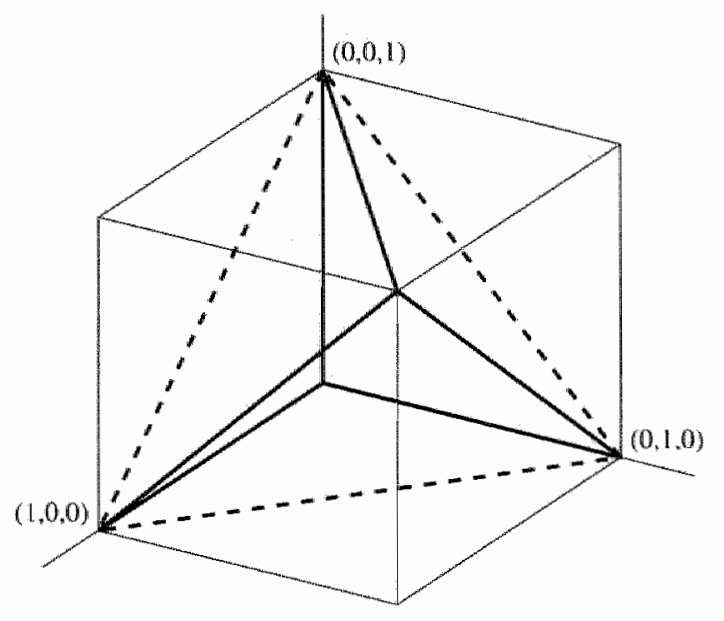

Figure 1.2: A finite set in $\mathbb{R}^{3}$ and its convex hull.

Consider, as an example, the set $S \subseteq \mathbb{R}^{3}$ containing the origin, all three unit vectors, and the point $(1,1,1)$, see Figure 1.2. The convex hull of $S$ is indicated by thick solid and dashed lines. It follows directly from the definition that the convex hull of a set is a convex set.

Definition 1.2 A set $\left\{x_{1}, \ldots, x_{k}\right\} \subseteq \mathbb{R}^{n}$ is linearly independent if the unique solution of $\sum_{i=1}^{k} \lambda_{i} x_{i}=\mathbf{0}$ is $\lambda_{i}=0$ for all $i=1, \ldots, k$.

Definition 1.3 A set $\left\{x_{1}, \ldots, x_{k}\right\} \subseteq \mathbb{R}^{n}$ is affinely independent if the unique solution of $\sum_{i=1}^{k} \lambda_{i} x_{i}=0$ and $\sum_{i=1}^{k} \lambda_{i}=0$ is $\lambda_{i}=0$ for all $i=1, \ldots, k_{i}$.

Notice that linear independence implies affine independence, but the opposite is not true. If $X \subseteq \mathbb{R}^{n}$ is linearly independent, then $X \cup\{0\}$ is affinely independent, but not limearly independent. Consider again the set $S=\left\{s_{0}, \ldots, s_{A}\right\}$ from Figure 1.2, where $s_{0}$ denotes the origin, $s_{1}, s_{2}, s_{3}$ denote the unit vectors, and $s_{4}=(1,1,1)$. This set is not affinely independent, since $-2 s_{0}+s_{1}+s_{2}+$ $s_{3}-s_{4}=0$, and hence it is not linearly independent either.

Definition 1.4 Given a vector $a \in \mathbb{R}^{n}, a \neq 0$, and a number $a_{0} \in \mathbb{R}$, the set $\left\{x \in \mathbb{R}^{n} \mid a^{T} x=a_{0}\right\}$ is a hyperplane, and the set $\left\{x \in \mathbb{R}^{n} \mid a^{T} x \leq a_{0}\right\}$ is called a halfspace. 
Definition 1.5 Given a set $S \subseteq \mathbb{R}^{n}$, a point $x \in \mathbb{R}^{n}$ is an affine combination of points in $S$ if there exists a finite set $\left\{s_{1}, \ldots, s_{k}\right\} \subseteq S$ and $\lambda \in \mathbb{R}^{k}$ with $\lambda_{1}+\ldots+\lambda_{k}=1$, such that $x=\lambda_{1} s_{1}+\ldots+\lambda_{k} s_{k}$. The affine hull of $S$, denoted by aff $(S)$, is the set of all points that are affine combinations of points in $S$.

If there exists a hyperplane $H \subseteq \mathrm{R}^{n}$ with $S \subseteq H$, then aff(S) is the intersection of all hyperplanes contaning $S$; if no such hyperplane exists, then aff $(S)=\mathbb{R}^{x_{5}}$.

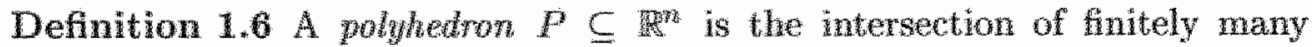
halfspaces, i.e.

$$
P=\left\{x \in \mathbb{R}^{n} \mid A x \leq b\right\}
$$

for some real $m \times n$ matrix $A$ and some $b \in Z^{m}$. A bounded polyhedron is called a polytope.

A hatfpace is a convex set. Since a polyhedron is the intersection of fintely many halfspaces, it is also convex. A polytope can also be defined as the convex hull of fintely many points in $\mathbb{R}^{*}$. The set conv(s) shown in tigure 1.2 is at polytope. It is contained in the halfspace $\left\{\left(x_{1}, x_{2}, x_{3}\right) \in \mathbb{R}^{3} \mid x_{1}+x_{2} \leq 2\right\}$.

Definition 1.7 A set $C \subseteq \mathbb{R}^{n}$ is a cone if $x \in C$ implies $\lambda x \in C$ for all $\lambda \geq 0$.

If there exists a finite set $X=\left\{x_{1}, \ldots, x_{k}\right\} \subseteq \mathbb{R}^{n}$ such that $C=\left\{\lambda_{1} x_{1}+\right.$ $\left.\ldots+\lambda_{k} x_{k} \mid \lambda_{1}, \ldots, \lambda_{k} \geq 0\right\}$, then $C$ is said to be generated by $X$. For any $x \in \mathbb{R}^{2 x}, x \neq 0$, the cone generated by $\{x,-x\}$ is simply a straight line through the origin. The cone generated by all unit vectors is the first orthant. Not all cones are generated by finite sets. Consider for instance the cone $\left\{x \in \mathbb{R}^{3} \mid x_{1}^{2}+x_{2}^{2} \leq x_{3}^{2}, x_{3} \geq 0\right\}$, which has the $x_{3}$-axis as axis of symmetry, and the lines $x_{1}=x_{3}$ and $x_{2}=x_{3}$ as descriptive lines, but there is no finite set that generates this cone. 
Definition 1.8 A polyhedron $P \subseteq \mathbb{R}^{m}$ has dimension $k$, denoted by $\operatorname{dim}(P)=$ $k$, if the maximum number of affinely independent points in $P$ is $k+1$. If $\operatorname{dim}(P)=n$ then $P$ is full dimensional.

The polytope conv $(S)$ in Figure 1.2 contains four affinely independent points, e.g. $\left\{s_{1}, \ldots, s_{4}\right\}$, and hence $\operatorname{dim}(\operatorname{conv}(S))=3$, so it is a full dimensional polytope.

Definition 1.9 If, for some $a \in \mathbb{R}^{n}, a \neq 0, a_{0} \in \mathbb{R}$, the polyhedron $P \subseteq \mathbb{R}^{n}$ is contained in the halfspace $\left\{x \in \mathbb{R}^{n} \mid a^{T} x \leq a_{0}\right\}$, then the inequality $a^{T} x \leq a_{0}$ is called a valid inequality for $P$.

Definition 1.10 Given a valid inequality $a^{T} x \leq a_{0}$ for a polyhedron $P \subseteq \mathbb{R}^{n}$, the set $F:=\left\{x \in P \mid a^{T} x=a_{0}\right\}$ is called a face of $P$. A face is said to be proper if $\varnothing \neq F \neq P$.

Notice that a face of a polyhedron is a polyhedron itself. Hence, we can refer to the dimension of a face. If $F$ is a proper face of $P$, then $0 \leq \operatorname{dim}(F) \leq$ $\operatorname{dim}(P)-1$. The empty face has dimension -1 .

Definition 1.11 Given a polyhedron $P \subseteq \mathbb{R}^{n}$ and a proper face $F$ of $P$. If $\operatorname{dim}(F)=\operatorname{dim}(P)-1$ then $F$ is called a facet. A face of dimension 0 is called a vertex or extreme point of $P$. A face of dimension 1 is called an edge of $P$.

The polytope conv $(S)$ has five vertices (namely all points of $S$ ), nine edges, and six facets. The facets are described by the inequalities $x_{k} \geq 0$ and $x_{i}+x_{j}-x_{k} \leq$ 1 for all distinct $i, j, k \in\{1,2,3\}$ with $i<j$.

Facet defining inequalities are necessary in the linear description of a polyhedron $P$. Besides being necessary, the facets are sufficient for the description of $P$, as is easily seen for the example in Figure 1.2 . 


\subsection{Graph theory}

In this section we provide some definitions from graph theory, and we introduce most of the notation used in this thesis. Concepts of graph theory not mentioned here can be found in Bondy and Murty [8].

Definition 1.12 The graph $G$ with vertex set $V$ and edge set $E$ is denoted by $G=(V, E)$. The graph $G$ is complete if $\{i, j\} \in E$ for all distinct $i, j \in V$.

The complete graph on $n$ vertices is usually denoted by $K_{n}=\left(V_{n}, E_{n}\right)$.

Definition 1.13 A walk in the graph $G=(V, E)$ is an alternating sequence

$$
v_{0}, e_{1}, v_{1}, e_{2}, v_{2}, \ldots, v_{k-1}, e_{k-1}, v_{k}
$$

of vertices $v_{0}, \ldots, v_{k} \in V$ and edges $e_{1}, \ldots, e_{k} \in E$, starting and ending with a vertex, such that $e_{i}=\left\{v_{i-1}, v_{i}\right\}$ for all $i=1, \ldots, k$. The length of a walk is the number of (not necessarily distinct) edges it contains.

Definition 1.14 A path in the graph $G=(V, E)$ is a walk in which no vertex is repeated. A cycle in $G$ is a walk of length at least 3 in which only the first and the last vertex coincide.

Since no vertices are repeated in a path, all edges of a path are distinct, as well as the edges of a cycle. Sometimes we denote a path or a cycle by its edge set, or by giving the order of its vertices.

Definition 1.15 A graph $G=(V, E)$ is connected if for each $u, v \in V$ there exists a path starting in $u$ and ending in $v$.

Definition 1.16 A graph $G=(V, E)$ is $k$-edge connected if deleting less than $k$ edges from $G$ leaves a connected graph. Analogously, $G$ is $k$-vertex connected if $|V| \geq k+1$ and deleting less than $k$ vertices from $G$ together with all edges incident to these vertices leaves a connected graph. 
Definition 1.17 Given are a graph $G=(V, E)$, two sets $S, T \subseteq V$, and a set $F \subseteq E$. The set of edges in $E$ incident to a vertex in $S$ and a vertex in $T$ is denoted by $E(S, T)$, i.e.

$$
E(S, T):=\{\{i, j\} \in E \mid i \in S, j \in T\}
$$

and $E(S):=E(S, S)$. The set of vertices in $V$ incident to an edge in $F$ is denoted by $V(F)$.

Definition 1.18 The graph $G^{r}=(U, F)$ is called a subgraph of $G=(V, E)$, denoted by $G^{\prime} \subseteq G$, if $U \subseteq V$ and $F \subseteq E(U)$. If $F=E(U)$ or $U=V(F)$ then $G^{\prime}$ is said to be induced by $U$ or $F$, respectively. A complete subgraph is called a clique.

Definition 1.19 A graph $G=(V, E)$ is triangle-free if it does not contain a cycle of length 3 , or equivalently, if it does not contain a complete subgraph on 3 vertices.

Definition 1.20 Given a graph $G=(V, E)$, the degree of a vertex $v \in V$ is the number of edges in $E$ incident to $v$.

Definition 1.21 Given a graph $G=(V, E)$, a subset $M \subseteq E$ is called a matching in $G$ if no vertex in $G^{\prime}=(V, M)$ has degree more than 1 .

Definition 1.22 Given are a graph $G=(V, E)$ and two distinct vertices $i, j \in V$ with $\{i, j\} \notin E$. If there exists a path of length $k$ in $G$ starting in $i$ and ending in $j$, then $\{i, j\}$ is a $k$-chord of $G$. The set of all $k$-chords of $G$ is denoted by $\mathrm{Ch}_{k}(E)$.

For a triangle-free graph $G=(V, E)$ the set of 2-chords is given by

$$
\{\{i, j\} \notin E \mid \exists k \in V:\{i, k\},\{j, k\} \in E\}
$$

We end this section with some notation used throughout this thesis. For a graph $G=(V, E)$, the incidence vector (also called characteristic vector) 
$\chi^{F} \in\{0,1\}^{|E|}$ of a subset $F \subseteq E$ is defined by $\chi_{i j}^{F}=1$ if $\{i, j\} \in F$ and $\chi_{i j}^{F}=0$ otherwise. Anialogously, the incidence vector $\chi^{U} \in\{0,1\}^{|V|}$ of a subset $U \subseteq V$ has $\chi_{i}^{U}=1$ if $i \in U$ and $\chi_{i}^{U}=0$ otherwise. If $x \in \mathbb{R}^{|E|}$ then

$$
x(F):=x^{T} \chi^{F}=\sum_{\{i, j\} \in F} x_{i j}
$$

The support of an inequality $a^{T} x \leq a_{0}$ in $\mathbb{R}^{|E|}$ is the graph $H=(V(F), F)$ incluced by the edges in $F:=\left\{\{i, j\} \in E \mid a_{i j} \neq 0\right\}$. Sometimes $F$ itself is called the support. Analogously, the positive support of an inequality $a^{T} x \leq$ $a_{0}$ is the graph $H^{+}=\left(V\left(F^{+}\right), F^{+}\right)$induced by the edges in $F^{+}:=\{\{i, j\} \in$ $\left.E \mid a_{i j}>0\right\}$.

\subsection{Related polyhedra}

The first two clustering problems described in Section 1.1 are examples of the clique partitioning problem. This section aims at putting the clique partitioning problem into perspective as far as the polyhedral approach is concerned. A precise definition of clique partitioning is as follows. Given a connected graph $G=(V, E)$ together with edge weights $w_{i j} \in \mathbb{R}$ for all $\{i, j\} \in E$, find a partition $\left\{V_{1}, \ldots, V_{p}\right\}$ of $V$ ( $p$ is not fixed), such that each subset $V_{i}$ $(i=1, \ldots, p)$ induces a clique in $G$ and the sum of the weights on the edges inside the subsets is maximized. This is expressed by the following integer program.

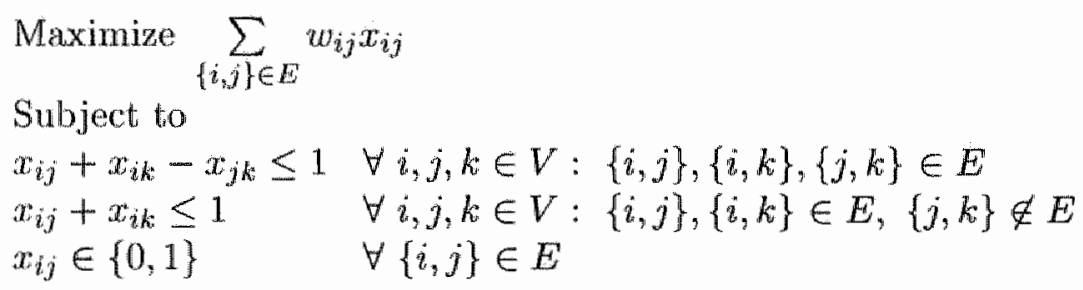

If $x_{i j}=1$ for some $\{i, j\} \in E$, then this edge belongs to the clique partition, otherwise it does not (a subset $F \subseteq E$ is called a clique partition if the components of the graph $G^{\prime}=(V, F)$ are cliques). The clique partitioning polytope $P(G)$ is the convex hull of all incidence vectors of clique partitions of $G$, or 
equivalently, the convex hull of all vectors satisfying (1.1). The clique partitioning polytope for complete graphs is studied intensively by Grotschel and Wakabayashi $[38,39,40]$. This polytope belongs to a family of related polyhedra, as shown in Figure 1.3, where we consider the case of complete graphs. The arrows in the figure indicate inclusion. Knowledge of the facial structure of one polyhedron provides knowledge of the structure of related polyhedra. Let us therefore discuss some connections between the various polyhedra of Figure 1.3.

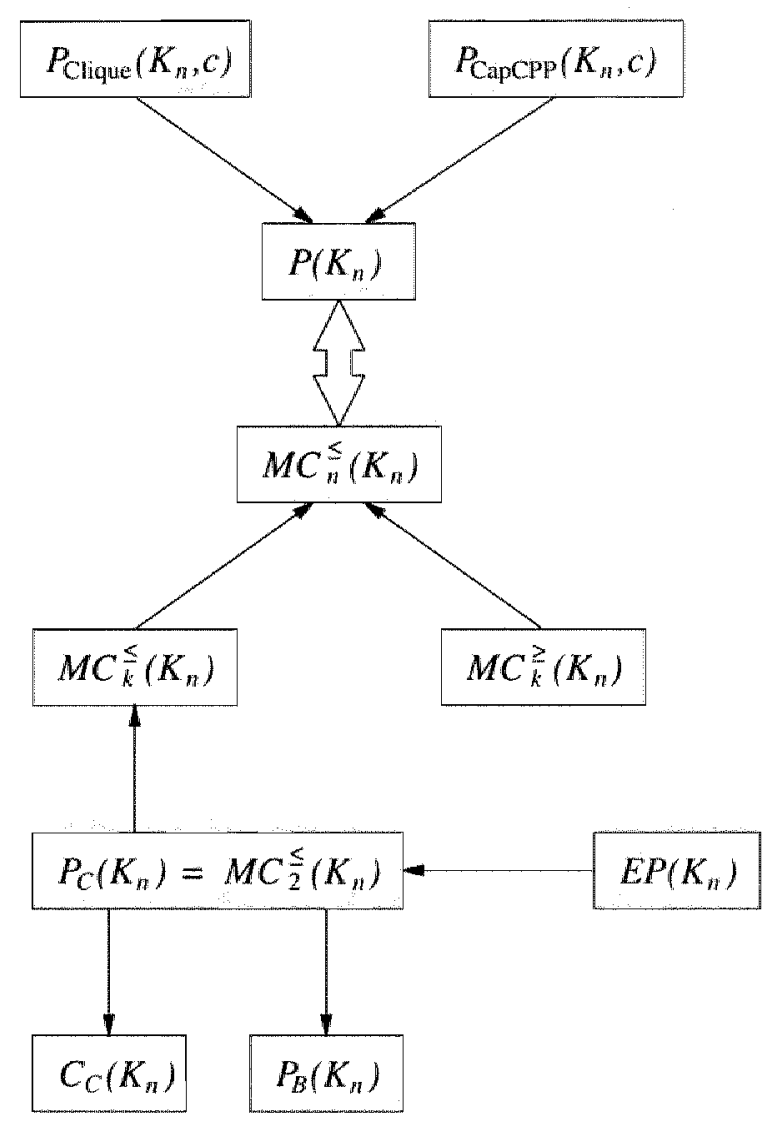

Figure 1.3: Polyhedra related to the clique partitioning polytope.

If $K_{n}=\left(V_{n}, E_{n}\right)$ and $F \subseteq E_{n}$ is a clique partition consisting of $k$ cliques, the complement $E_{n} \backslash F$ is a multicut of $K_{n}$ with $k$ shores. The convex hull of all multicuts with at most (at least) $k$ shores is denoted by $M C_{k}^{\leq}\left(K_{n i}\right)$ 
$\left(M C_{k}\left(K_{n}\right)\right)$. The multicut polytope $M C_{n}^{\leq}\left(K_{n}\right)$ contains all multicuts of $K_{n}$, and it is nothing but an affine transformation of $P\left(K_{n}\right)$. Namely, if $x \in \mathbb{R}^{\left|E_{n}\right|}$ is the incidence vector of a clique partition, then $1-x$ is the incidence vector of a multicut, and vice versa, hence

$$
M C_{n}^{\leq}\left(K_{n}\right)=\left\{\mathbf{1}-x \mid x \in P\left(K_{n}\right)\right\}
$$

This also implies that if $a^{T} x \leq a_{0}$ defines a face (facet) of $P\left(K_{n}\right)$ the inequality $a^{T}(1-x) \leq a_{0}$ defines a face (facet) of $M C_{n}^{\leq}\left(K_{n}\right)$. Deza et al. [25] study these general multicut polytopes, whereas in one paper Chopra and Rao [13] study the polytope $M C_{k}^{\geq}\left(K_{n}\right)$, and in two later papers $[14,15]$ they study the polytope $M C_{k}^{\leq}\left(K_{n}\right)$.

A cut in a graph $G=(V, E)$ is a subset of the edges that can be written as $E(U, V \backslash U)$ for some $U \subseteq V$. The cut polytope $P_{C}\left(K_{n}\right)$ is the convex hull of all incidence vectors of cuts of $K_{n}$, and it is not hard to see that it is a special case of the multicut polytope: $P_{C}\left(K_{n}\right)$ is simply equal to $M C_{2}^{\leq}\left(K_{n}\right)$. The cut polytope is investigated by Barahona and Mahjoub [6]. An even more special case is the equicut or equipartition polytope $\operatorname{EP}\left(K_{n}\right)$. This polytope is the convex hull of all cuts (not multicuts) of $K_{n}$ dividing $V_{n}$ into two subsets containing $\left\lceil\frac{1}{2}\left|V_{n}\right|\right\rceil$ and $\left\lfloor\frac{1}{2}\left|V_{n}\right|\right\rfloor$ vertices. The equicut polytope is studied by Conforti et all. [20,21], and by Laurent and de Souza [48]. Deza and Laurent $[23,24]$ investigate the structure of the cut cone $C_{C}\left(K_{n}\right)$ generated by the incidence vectors of all cuts of $K_{n}$ instead of the cut polytope $P_{C}\left(K_{n}\right)$. Studying the facets of the cut cone is, in some sense, equivalent to studying the facets of the cut polytope. Barahona and Mahjoub [6] proved that the cut polytope has the following property: a description of the facets that contain any particular extreme point gives the description of the whole polytope. For this reason, it is sufficient to study the facets that contain the origin, i.e. the facets of the cut cone $C_{C}\left(K_{n}\right)$. Another polytope that contains $P_{C}\left(K_{n}\right)$ is the bipartite subgraph polytope $P_{B}\left(K_{n}\right)$. If $F \subseteq E_{n}$ is a cut in $K_{n}$, and $F^{\prime} \subseteq F$, then the graph $\left(V_{n}, F^{\prime}\right)$ is bipartite. On the other hand, if $F^{\prime} \subseteq E_{n}$ induces a bipartite subgraph of $K_{n}$, then $F^{\prime}$ can be extended to a cut of $K_{n}$. The bipartite subgraph polytope $P_{B}\left(K_{n}\right)$ is the convex hull of all incidence vectors of bipartite subgraphs of $K_{n}$.

Additional constraints on the clique partitioning problem give rise to other polytopes contained in $P\left(K_{n}\right)$. For instance, if the size of the clusters is restricted to $c \in \mathbb{N}$ we arrive at the capacitated clique partitioning problem, 
and the associated polytope is denoted by $P_{\text {Capcpp }}\left(K_{n}, c\right)$. Its structure is investigated by Faigle et al. [27]. Restricting the number of clusters to one, and restricting the size of the cluster to $c \in \mathbb{N}$ leads to the maximal edgeweighted clique problem, whose polytope $P_{\text {Clique }}\left(K_{n}, c\right)$ is studied by Dijkhuizen and Faigle [26]. An extended formulation for the edge-weighted clique problem is given by Park et al. [62] and by Macambira and de Souza [52]. The extended formulation has, next to the variables for all edges of the graph, also variables for the vertices of the graph. Although these additional variables are not required to arrive at a correct integer programming formulation, it turns out that the linear programming relaxation of the extended formulation gives a much better performance when solving instances of the edge-weighted clique problem by means of a branch-and-cut algorithm.

A more general problem is obtained if, apart from the edge weights, also costs $c_{v} \geq 0\left(v \in V_{n}\right)$ are given, together with a number $b \geq 0$ and an integer $p \in \mathbb{N}$, and the objective is to find a partition $\left\{V_{1}, \ldots, V_{p}\right\}$ of $V_{n}$ that solves

$$
\begin{array}{ll}
\operatorname{Max}(\operatorname{Min}) & \sum_{k=1}^{p} \sum_{\{i, j\} \in E\left(V_{k}\right)} w_{i j} \\
\text { Subject to } & \sum_{v \in V_{k}} c_{v} \leq b \quad \forall k=1, \ldots, p
\end{array}
$$

In other words, the clustering problem is to partition the vertex set of $K_{n}$ into $p$ clusters, some of which may be empty, such that the sum of the vertex costs of each cluster is bounded from above by $b$, while maximizing (minimizing) the sum of the weights on the edges inside clusters. This problem is studied by Johnson et al. [46] and Ferreira et al. [29].

If all vertex costs are equal to zero, all edge weights are nonnegative, and $p=2$ formulation (1.2) is the well known MAX(MIN)CUT problem. Note that while MINCUT is solvable in polynomial time [2], MAXCUT is NP-hard [31]. If $p=2$, $c_{v}=1$ for all $v \in V_{n}$ and $b=\left\lceil\frac{1}{2}\left|V_{n}\right|\right\rceil$, then (1.2) is again the equipartition problem studied by Conforti et al. [20,21] and by Laurent and de Souza [48].

A different formulation for the clique partitioning problem is the following. Let $\left\{y^{1}, \ldots, y^{q}\right\} \subseteq \mathbb{R}^{\left|V_{n}\right|}$ be the set of incidence vectors of all nonempty subsets of $V_{n}$, and let $\bar{d}_{k}$ be the total weight of all edges between vertices in cluster $k$ 
$(k=1, \ldots, q)$. The clique partitioning problem can also be formulated as

$$
\begin{array}{lll}
\text { Maximize } & \sum_{k=1}^{q} d_{k} \lambda_{k} & \\
\text { Subject to } & \sum_{k=1}^{q} y_{i}^{k} \lambda_{k}=1 \quad \forall i \in V_{n} \\
& \lambda_{k} \in\{0,1\} \quad \forall k=1, \ldots, q
\end{array}
$$

Obviously, all variables $\lambda_{k}$ with $d_{k}<0(k=1, \ldots, q)$ can be deleted from the formulation above, since these variables are all equal to zero in any optimal solution. The equalities in (1.3) can be relaxed to "less than or equal to" constraints. Formulation (1.3) has an exponential number of columns, and listing all of them is impractical, if not impossible. Instead, one usually starts with a few columns, and adds new columns as required. A possible set of starting columns is the set of all incidence vectors corresponding to a single vertex of $V_{n}$, i.e. start with $\left|V_{n}\right|$ unit vectors as columns. This ensures that (1.3) always has a feasible solution. Let $\pi_{i}\left(i \in V_{n}\right)$ be the dual variables for (1.3), then the subproblem that must be optimized to generate the most attractive column is

$$
\operatorname{Maximize} \sum_{\substack{i, j \in V_{n} \\ i \neq j}} w_{i j} z_{i} z_{j}-\sum_{i \in V_{n}} \pi_{i} z_{i}
$$

where $z_{i} \in\{0,1\}$ for all $i \in V$. So, finding new columns comes down to solving an unconstrained quadratic $\{0,1\}$-programming problem, known as the boolean quadratic problem $[1,61]$. The column generation approach is successfully used by Johnson et al. [46] for solving clustering problems arising from compiler design.

\subsection{How to read this book}

This thesis is best read from cover to cover, but if that is not possible for no matter what reason it is highly recommended to read at least the entire first chapter. This section will then function as a guide to the thesis and help to find the most interesting parts. 
Chapter 2 starts with defining the clique partitioning polytope, and presenting some basic results on its dimension and on properties of facet defining inequalities. Next, two different lifting theorems are described. The first one is a special kind of zero lifting for clique partitioning, while the second one describes how, under some conditions, simple facet defining inequalities can be combined to give more complex facet defining inequalities. This procedure is called patching, and it is used frequently for proving that a given valid inequality for the clique partitioning polytope is facet defining. In many situations patching yields much shorter and more intuitive proofs for 'facetness'. This is illustrated for a very broad class of complex inequalities that define facets of the clique partitioning polytope of arbitrary graphs.

Chapter 3 deals with the clique partitioning polytope of complete graphs. It starts with listing all known facets of these polytopes. Next, several new classes of facets are described. In particular, all facet defining inequalities with right-hand side equal to 1 or 2 are classified. Furthermore, some new variants of patching are given.

Chapter 4 gives complete descriptions of the clique partitioning poly tope associated with the complete graphs on 4 and 5 nodes, and a partial description in the case of 6 nodes. The polytope $P\left(K_{5}\right)$ contains three classes of facets that are not described in literature. We present generalizations of these classes for complete graphs on more than 5 nodes.

Chapter 5 gives a number of different measures for the 'strength' of an inequality, i.e. a measure for the potential usefulness of an inequality in a branchand-cut algorithm. The strength of all known facets of $P\left(K_{n}\right)$ with $n \leq 6$ is established, as well as the strength of several classes of facets for complete graphs with an arbitrary number of vertices.

Chapter 6 deals with the complexity of separating facet defining inequalities for the clique partitioning polytope. The separation problem for classes containing only a polynomial number of facets can of course be solved efficiently, and also for the class of (bicycle) wheel inequalities efficient separation algorithms are known. We show that the separation problem for some of the classes described in Chapter 2 and 3 is NP-hard, whereas it is polynomially solvable for a class of facets obtained from patching.

Chapter 7 is devoted to the following clustering problem: given a graph $G=$ $(V, E)$ and an integer $c \in \mathbb{N}$, what is the maximal cardinality of a subset 
U $\subseteq$ such that he graph $G^{f}=(U, E(U))$ has no component containing more than $c$ vertices. Several integer programming formulations for this problem have appeared in literature, and all these formulations assume that an upper bound on the naximal number of components of $G^{f}$ is given. We present an integer programming lormulation that does not suffer from this assumption, and report on its value in practise.

Chapter 8 extends the patching procedure of Chapter 2 for the clique partitoning polytope to a procedure for general $\{0,1\}$-polytopes. It is shown how that procedure can be used to derive facets for the independent set polytope, the knapsack polytope, and the traveling salesman polytope. 


\section{Chapter 2}

\section{Clique partitioning}

\section{$2.1 \quad$ Introduction}

Polytopes arising from mathematical programming problems are usually closely related to a combinatorial object, like a graph or a scheduling problem. Exposing the structure of such a polytope can be done in several ways. One possibility is describing various classes of valid inequalities and equations, and determining which inequalities define facets. Another possibility lies in explicit procedures to derive facets from facets, such as the node cloning and edge splitting procedures for the travelling salesman problem, or implicit procedures, like sequential and simultaneous lifting. A third approach is investigating the structure of 'small polytopes', for instance by enumerative methods, and stating when facets of these small polytopes give rise to facets of larger polytopes. This technique is related to lifting. For investigating the clique partitioning polytope we use all three methods. The first one, identifying new classes of facets, is postponed to the next chapter. In this chapter we prove two lifting theorems for the clique partitioning polytope of arbitrary graphs. In Section 2.2 we give sufficient conditions for zero lifting, and in Section 2.3 we show how facets of small polytopes can be used to recognize more complex facets of larger polytopes.

We recall the definition of clique partitioning from Chapter 1 . Given is a (connected) graph $G=(V, E)$ with edge weights $w_{i j} \in \mathbb{R}$ for all $\{i, j\} \in E$. A subset $F \subseteq E$ of the edges is called a clique partition if the components of 
the graph $G^{\prime}=(V, F)$ are cliques. The problem is to find a clique partition of maximum weight, which is equivalent to solving the following integer program.

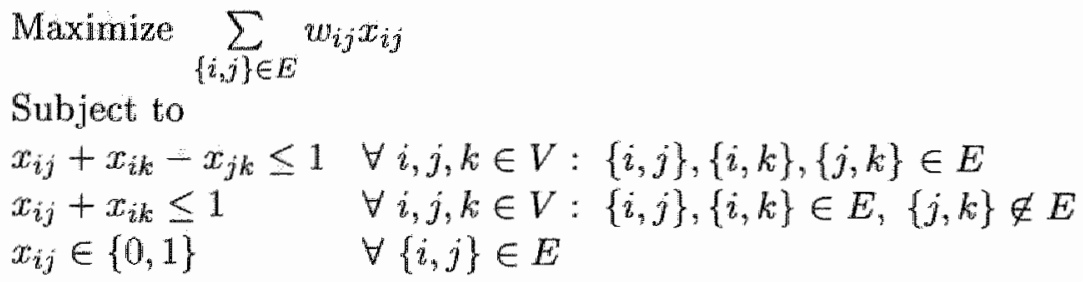

If $x_{i j}=1$ for some $\{i, j\} \in E$, then this edge belongs to the clique partition, otherwise it does not. The constraints in (2.1) are named after their support: they are called triangle inequalities and $K_{1,2}$-inequalities, respectively. The polyhedral approach to clique partitioning was initiated in a paper by Marcotorchino and Michaud [53], in which they present a slightly different formulation for this problem in the case of complete graphs. For general graphs $G$ the clique partitioning polytope $P(G)$ is the convex hull of all incidence vectors of clique partitions of $G$. The polytope $P(G)$ has dimension $|E|$, since it contains the zero vector and all unit vectors, and this also implies that the inequalities $x_{i j} \geq 0(\{i, j\} \in E)$ define facets, which are called trivial facets. However, the triangle inequality $x_{i j}+x_{i k}-x_{j k} \leq 1$ and the $K_{1,2}$-inequality $x_{i j}+x_{i k} \leq 1(i, j, k \in V)$ define facets if and only if for all $\{i, l\} \in E$ at least one of the edges $\{j, l\}$ and $\{k, l\}$ belongs to $E$. The nonnegativity constraints, the triangle inequalities, and the $K_{1,2}$-inequalities together are called the basic inequalities. The upper bound constraint $x_{i j} \leq 1(\{i, j\} \in E)$ defines a facet of $P(G)$ if and only if $\{i, j\}$ is an isolated edge, i.e. $\{i, j\} \subseteq V$ is the vertex set of a component of $G$.

Grötschel and Wakabayashi [39] also investigate the facial structure of the clique partitioning polytopes associated with complete graphs. They present several classes of facets of $P\left(K_{n}\right)$, including the following ones. The main purpose for presenting these facets here is because we use them as an illustration for our lifting theorems.

Theorem $2.1[39]$ Let $S, T \subseteq V_{n}$ be two nonempty disjoint subsets of $V_{n}$. The \&-partition inequality

$$
x\left(E_{n}(S, T)\right)-x\left(E_{n}(S)\right)-x\left(E_{n}(T)\right) \leq \min \{|S|,|T|\}
$$


is valid for $P\left(K_{n}\right)$. It defines a facet if and only if $|S| \neq|T|$.

Given $S, T \subseteq V_{n}$, the 2-partition inequality induced by $S$ and $T$ is sometimes called an $(S, T)$-inequality for short.

Theorem 2.2 [39] Let $C \subseteq E_{n}$ be a cycle of length at least 5 in $K_{n}$, and let $\vec{C}=\mathrm{Ch}_{2}(C)$. The 2-chorded cycle inequality

$$
x(C)-x(\bar{C}) \leq\left\lfloor\frac{1}{2}|C|\right\rfloor
$$

is valid for $P\left(K_{n}\right)$. It defines a facet if and only if $|C|$ is odd.

In the remainder of this chapter we are not searching for new facets, but instead we prove two lifting theorems for the clique partitioning polytope, which provide sufficient conditions for a valid inequality to be facet defining. Furthermore, to illustrate these results, we present generalizations of the 2partition inequalities for arbitrary graphs, and we give sufficient conditions for these inequalities to be facet defining.

\subsection{Zero lifting}

A natural question to ask is whether facets of $P\left(G^{\prime}\right)$ give rise to facets of $P(G)$ when $G^{\prime}$ is a subgraph of $G$. Grötschel and Wakabayashi [39] show that facets of $P\left(K_{m}\right)$ can be lifted (zero lifting) to facets of $P\left(K_{n}\right)$ for $n>m$, provided that there is a least one clique partition whose incidence vector belongs to the facet, and that has a single vertex as a clique. Theorem 2.5 describes a lifting technique that can be applied to arbitrary graphs. The idea used in this theorem comes down to the following: if $G^{\prime}=(U, E(U))$ is the subgraph of $G=(V, E)$ induced by $U \subseteq V$, and if $\{u, v\} \in E$ for all $u \in U$ and $v \in V \backslash U$, then any facet defining inequality of $P\left(G^{\prime}\right)$ can be extended in a trivial way to a facet defining inequality of $P(G)$. When applied to complete graphs this theorem implies that zero lifting is always possible. The proof of Theorem 2.5 requires Lemma 2.4 and the next definition. 
Definition 2.3 Let $G=(V, E)$ be a graph, $F \subseteq E$, and let $x \in \mathbb{R}^{|E|}$. The projected vector $x_{F} \in \mathbb{R}[E]$ is defined by

$$
\left(x_{F}\right)_{i j}= \begin{cases}x_{i j} & \text { if }\{i, j\} \in F \\ 0 & \text { otherwise }\end{cases}
$$

The truncated vector $\bar{x}_{F} \in \mathbb{R}^{|F|}$ is defined by $\left(\bar{x}_{F}\right)_{i j}:=x_{i j}$ for all $\{i, j\} \in F$.

Lemma 2.4 Let $G=(V, E)$ be a connected graph, and let $A$ be the vertexedge incidence matrix of $G$. If $G$ is bipartite, then $A$ has rank $|V|-1$, otherwise $A$ has rank $|V|$.

Proof For all $e \in E$ denote by $a_{e}$ the column of $A$ indexed by $e$. The vertex-edge incidence matrix of any spanning tree of $G$ has rank $|V|-1$, so $\operatorname{rank}(A) \geq|V|-1$. Two cases can occur.

Case 1: $G$ is bipartite. Let $T=(V, D)$ be a spanning tree of $G$, and consider any edge $e_{1} \in E \backslash D$. The graph $\left(V, D \cup\left\{e_{1}\right\}\right)$ contains an even cycle $\left\{e_{1}, \ldots, e_{k}\right\}$, and

$$
\sum_{i=1}^{k}(-1)^{k} \cdot a_{e_{i}}=\mathbf{0}
$$

Hence $a_{e_{1}}$ is a linear combination of $a_{e_{2}}, \ldots, a_{e_{k}}$.

Case 2: $G$ is not bipartite. Then $G$ contains an odd cycle $\left\{e_{1}, \ldots, e_{k}\right\}$. Choose a spanning tree $T=(V, D)$ of $G$ containing the edges $e_{2}, \ldots, e_{k}$, but not containing edge $e_{1}$. The vertex-edge incidence matrix of $T$ has rank $|V|-1$, and $a_{e_{1}}$ is not a linear combination of $a_{e_{2}}, \ldots, a_{e_{k}}$. Hence, $a_{e_{1}}$ is not a linear combination of the columns associated with the edge set $D$ of $T$ (as is seen by a straightforward inductive argument). Therefore $A$ has rank $|V|$.

\section{Theorem 2.5 (Zero lifting)}

Given are a graph $G=(V, E)$ and an inequality $a^{T} x \leq a_{0}$ valid for $P(G)$. Let $U$ be a proper subset of $V$ such that $E(U)$ includes the support $E_{a} \subseteq E$ of $a^{T} x \leq a_{0}$ and the following two conditions hold: 
(i) The truncated inequality $\vec{a}_{E(U)}^{T} \bar{x}_{E(U)} \leq a_{0}$ defines a nontrivial facet of $P(U, E(U))$,

(ii) $E(U, V \backslash U)$ has full size $|U| \cdot|V \backslash U|$.

Then $a^{T} x \leq a_{0}$ defines a facet of $P(G)$.

Proof Given the facet $\bar{F}=\left\{\bar{x} \in P(U, E(U)) \mid \vec{a}^{T} \ddot{x}=a_{0}\right\}$ of $P(U, E(U))$, the lifted face $F=\left\{x \in P(G) \mid a^{T} x=a_{0}\right\}$ is contained in some facet $F_{d}$ of $P(G)$, which may be defined by a valid inequality of the form $d^{T} x \leq a_{0}$. The clique partitions of $(U, E(U))$ can be regarded as clique partitions of $G$ not containing any of the edges in $E \backslash E(U)$. The corresponding characteristic vectors $\ddot{x} \in$ $P(U, E(U))$ are thereby turned into expanded vectors $x^{T}=\left(\bar{x}^{T}, 0^{T}\right)$ in $P(G)$ by adding a zero vector of length $p=|E \backslash E(U)|$. Writing $d^{T}=\left(b^{T}, c^{T}\right)$, where $c$ is a vector of length $p$, we then obtain

$$
\bar{a}^{T} \bar{x}=a_{0}=d^{T} x=b^{T} \bar{x} \quad \forall \bar{x} \in \bar{F}
$$

and consequently $b=\bar{a}$. Hence it remains to show that $c=0$. It is obvious that $c_{i j}=0$ for all $\{i, j\} \in E(V \backslash U)$. Now, let $\bar{x} \in \vec{F}$ be the characteristic vector of a clique partition, and let $S_{1}, \ldots, S_{r} \subseteq U$ be the partition of $U$ induced by $\bar{x}$. For all $k=1, \ldots, r$ let $v^{k}$ be the characteristic vector of length $|U|$ of $S_{k}$, i.e. $v_{i}^{k}=1$ if $i \in S_{k}$ and $v_{i}^{k}=0$ otherwise. Let $u \in V \backslash U$. The clique partition $\vec{x}$ can be extended to $r$ distinct clique partitions of $G$ by adjoining vertex $u$ to exactly one of the sets $S_{k}(k=1, \ldots, r)$. This is possible due to condition (ii). Their characteristic vectors $y^{1}, \ldots, y^{r}$, as well as $x$, all belong to the lifted face $F$ (since $V\left(E_{a}\right) \subseteq U$ ), and thus also to the facet $F_{d}$ of $P(G)$, whence

$$
\sum_{i \in S_{k}} c_{u i}=d^{T}\left(y^{k}-x\right)=0 \quad \forall k=1, \ldots, r
$$

or equivalently

$$
c_{u}^{T} v^{k}=0 \quad \forall k=1, \ldots, r
$$


where the vector $c_{n d}$ is indexed by the vertices of $U$. Iet $\bar{x}^{k}=\chi^{E\left(S_{k}\right)}(k=$ $1, \ldots, r)$, so that $\vec{x}=\bar{x}^{1}+\ldots+\bar{x}^{r}$, and let $A$ denote the vertex-edge incidence matrix of the graph $(U, E(U))$. We obtain

$$
A \ddot{x}^{k}=\left(\left|S_{k}\right|-1\right) \cdot v^{k} \quad \forall k=1, \ldots, r
$$

and therefore

$$
c_{u}^{T} A \bar{x}^{k}=\left(\left|S_{k}\right|-1\right) \cdot c_{u}^{T^{n}} v^{k}=0 \quad \forall k=1, \ldots, r
$$

Since $\bar{F}$ is a nontrivial facet of $P(U, E(U))$ there exist $m:=|E(U)|$ linearly independent vectors $w_{i} \in \vec{F}$ of clique partitions of $(U, E(U))(i=1, \ldots, m)$ Each $w_{i}$ induces a partition of $U$ into $S_{i}^{1}, \ldots, S_{i}^{r_{i}}$. Let $w_{i}^{j}:=\chi^{E\left(S_{i}^{j}\right)}(i=$ $\left.1, \ldots, m, j=1, \ldots, r_{m}\right)$, and define the matrix $W$ as

$$
W=\left[\begin{array}{l|l|l}
w_{1}^{1} \ldots w_{1}^{r_{1}} & \ldots \ldots & w_{m}^{1} \ldots w_{m}^{r_{m}}
\end{array}\right]
$$

Then $W$ satisfies $c_{u}^{T} A W=0$. The matrix $W$ has rank $m$ since $w_{1}, \ldots, w_{r_{1}}$ belong to the linear space generated by the columns of $W$. We distinguish tw cases.

Case 1: The graph $(U, E(U))$ contains an odd cycle. From Lemma $2.4 \mathrm{i}$ follows that $A$ has rank $|U|$, so the product matrix $A W$ has full row rank because both $A$ and $W$ have full row rank. We therefore conclude that $c_{w}=\mathrm{c}$ and hence $c=0$.

Case 2: The graph $(U, E(U))$ contains no odd cycle. Then $(U, E(U))$ is bipas tite, and hence any clique in a clique partition contains at most two vertices It follows that a clique partition of $(U, E(U))$ is nothing but a matching, $c$ equivalently, $P(U, E(U))$ is exactly the matching polytope corresponding t $(U, E(U))$. Therefore the inequality $\bar{a}^{T} \bar{x} \leq a_{0}$ is a so-called degree constrain

$$
\sum_{j:\{j, s\} \in E(J)} x_{s j} \leq 1
$$




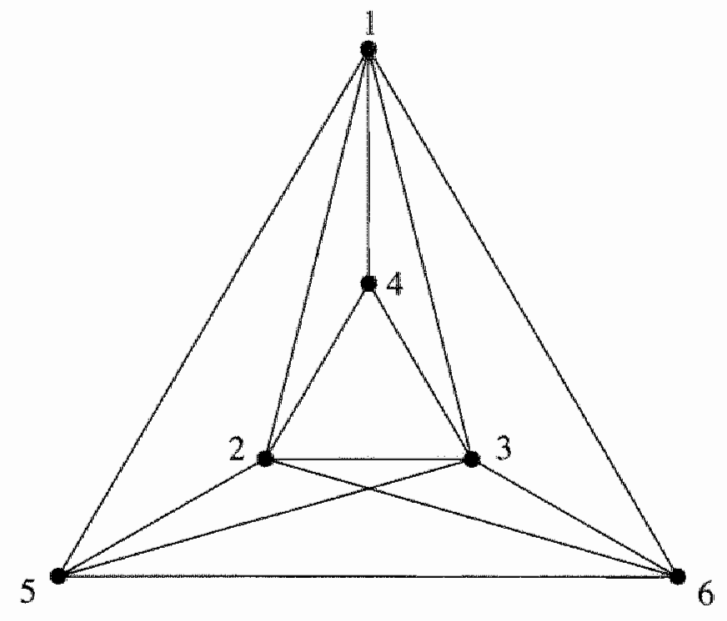

Figure 2.1: The graph of Example 2.6.

for some $s \in U$ with degree at least 2 (since these degree constraints are the only nontrivial facets of the matching polytope of a bipartite graph). To proceed, we exhibit $|E|$ affinely independent solutions that satisfy (2.4) at equality. Since (2.4) defines a facet of $P(U, E(U))$ there exist $m$ affinely independent solutions containing only edges in $E(U)$. It is easy to verify that this set of solutions together with the characteristic vectors of the following clique partitions is still affinely independent: selecting distinct vertices $t, t^{\prime} \in U$ adjacent to $s$, consider

$$
\begin{array}{ll}
\{\{s, t\},\{v, w\}\} & \forall\{v, w\} \in E(V \backslash U) \\
\{\{s, t\},\{u, v\}\} & \forall u \in U \backslash\{s, t\}, v \in V \backslash U \\
\left\{\left\{s, t^{\prime}\right\},\{t, v\}\right\} & \forall v \in V \backslash U \\
E(\{s, t, v\}) & \forall v \in V \backslash U
\end{array}
$$

Since all these solutions lie in the face $F$, and thus also in $F_{d}$, it follows that $d^{T}=\left(b^{T}, c^{T}\right)=\beta \cdot\left(\bar{a}^{T}, 0^{T}\right)$ for some $\beta>0$, and hence $c=0$.

The following example serves as an illustration of Theorem 2.5 .

Example 2.6 Let $G$ be the graph shown in Figure 2.1, and let $x_{12}+x_{13}-x_{23} \leq$ 1 be the given valid inequality. Since this inequality defines a facet for $K_{3}$ (so 


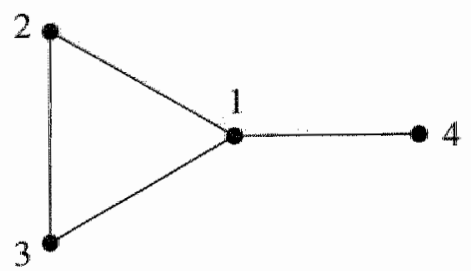

Figure 2.2: The graph of Example 2.7.

(i) is fulfilled), and each edge between the node sets $\{1,2,3\}$ and $\{4,5,6\}$ belongs to $E$ (so (ii) is fulfilled), the inequality $x_{12}+x_{13}-x_{23} \leq \mathbb{1}$ is facet defining for $P(G)$.

The relevance of condition (ii) in Theorem 2.5 follows from the following exanple.

Example 2.7 Let $G$ be the graph shown in Figure 2.2.

(a) Let $x_{12}+x_{13}-x_{23} \leq 1$ be the given valid inequality. Condition (ii) is not fulfilled, because the edges $\{2,4\}$ and $\{3,4\}$ are missing, and indeed the inequality does not define a facet of $P(G)$ because it is implied by $x_{12}+x_{13}+$ $x_{14}-x_{23} \leq 1$.

(b) Let $x_{12}-x_{13}+x_{23} \leq 1$ be the given valid inequality. Although condition

(ii) is not fulfilled, this inequality defines a facet of $P(G)$.

Example $2.7 \mathrm{~b}$ implies that condition (ii) is not a necessary condition. However, it is always fulfilled in the case of complete graphs. In that case, Theorem 2.5 is equivalent to the following corollary.

Corollary 2.8 Let $b^{T} y \leq b_{0}$ be a facet defining inequality for the polytope $P\left(K_{m}\right)(m \geq 3)$. Then for every $n>m$, the inequality $a^{T} x \leq a_{0}=b_{0}$, where $a^{T}=\left(b^{T}, 0^{T}\right)$ and $x^{T}=\left(y^{T}, 0^{T}\right)$, defines a facet of $P\left(K_{n}\right)$.

This corollary confirms that the condition employed by Grötschel and Wakabayashi [39] to ensure that lifted facets remain facets is superfluous. 


\section{$2.3 \quad$ Recognizing facets}

In this section we present our second lifting theorem. Intuitively, the theorem says that if one is able to cover in a certain way the support of a given valid inequality with known facets, then the inequality is facet defining. The proof is based on the following idea: given a valid inequality $a^{T} x \leq a_{0}$ and its support $E_{a}$, it is possible to extend the set of affinely independent solutions corresponding to some facet defining inequality 'contained' in the support $E_{a}$; even more, it is possible to combine the extended sets of affinely independent solutions of different facets contained in the support $E_{a}$ to show that the given inequality defines a facet.

In order to give a precise formulation of the lifting theorem, we need some additional notation. Given the graph $G=(V, E)$, for each edge set $D \subseteq E$ we define

$$
\begin{aligned}
& L_{G}(D):=\left\{x \in P(G) \cap\{0,1\}^{|E|} \mid x_{i j}=0 \forall\{i, j\} \in E \backslash D\right\} \\
& R_{G}(D):=\left\{x \in P(G) \cap\{0,1\}^{|E|} \mid x_{i j}=0 \forall\{i, j\} \cap V(D) \neq \varnothing\right\}
\end{aligned}
$$

In words, $L_{G}(D)$ is the set of clique partitions of $G$ containing only edges in $D$, and $R_{G}(D)$ is the set of clique partitions of $G$ containing neither edges in $D$ nor edges incident to $V(D)$. Notice that, if $x \in L_{G}(D)$ and $y \in R_{G}(D)$, then $x+y \in P(G)$. The following example illustrates this concept.

Example 2.9 Let $K_{5}=\left(V_{5}, E_{5}\right)$ be the complete graph on 5 vertices, and let $D=E_{5}(\{1,4,5\}$ ) (see Figure 2.3), then

$$
\begin{aligned}
& L_{G}(D)=\left\{\varnothing,\{1,4\},\{1,5\},\{4,5\}, E_{5}(\{1,4,5\})\right\} \\
& R_{G}(D)=\{\varnothing,\{2,3\}\}
\end{aligned}
$$

It is easy to see by inspection that for all $x \in L_{G}(D)$ and $y \in R_{G}(D)$ the vector $x+y$ belongs to $P(G)$. $\square$

Next, we define the load and the residual of an edge set with respect to a given inequality. 


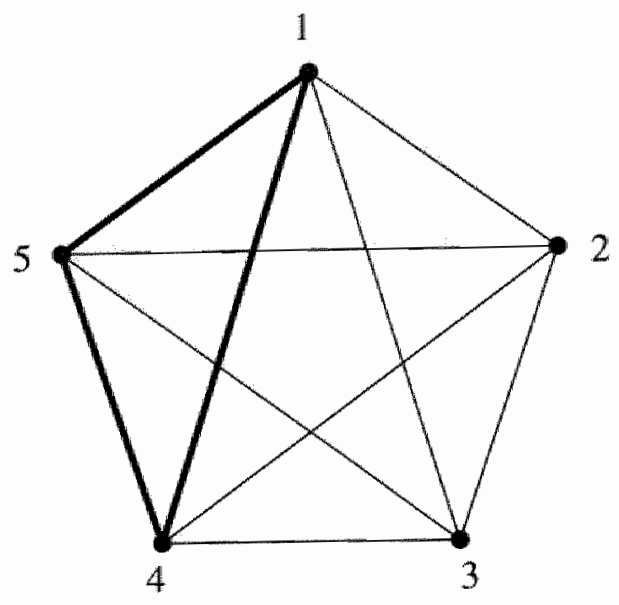

Figure 2.3: The graph $K_{5}$ and the edge set $D=\{\{1,4\},\{1,5\},\{4,5\}\}$.

Definition 2.10 Let $G=(V, E)$ be a graph, $D \subseteq E$, and let $a^{T} x \leq a_{0}$ be a valid inequality for $P(G)$. The load and the residual of $D$ with respect to $a^{T} x \leq a_{0}$ are given by

$$
\begin{aligned}
\operatorname{load}_{G}(a, D) & :=\max \left\{a^{T} x \mid x \in L_{G}(D)\right\} \\
\operatorname{res}_{G}(a, D) & :=\max \left\{a^{T} x \mid x \in R_{G}(D)\right\}
\end{aligned}
$$

Notice that the load and the residual are always nonnegative since both $L_{G}(D)$ and $R_{G}(D)$ contain the zero vector. Let us again illustrate the idea by means of an example.

Example 2.11 Consider again the complete graph $K_{5}$ on 5 vertices together with the edge set $D=E_{5}(\{1,4,5\})$, and let $a^{T} x \leq a_{0}$ denote the $(S, T)$ inequality induced by $S=\{1,2\}$ and $T=\{3,4,5\}$. The load of $D$ with respect to $a^{T} x \leq a_{0}$ is 1 , and the maximum of $\left\{a^{T} x \mid x \in L_{G}(D)\right\}$ is attained, for instance, by the clique partition $E_{5}(\{1,4,5\})$. For the residual we have res $_{K_{5}}(a, D)=1$, since $\{2,3\} \in R_{K_{5}}(D)$ and $a_{23}=1$.

The following inequality is valid by definition.

$$
\max _{x \in L_{G}(D)} a^{T} x+\max _{y \in R_{G}(D)} a^{T} y=\max _{z \in P(G)} a^{T} z \leq a_{0}
$$


So, $\operatorname{load}_{G}(a, D)+\operatorname{res}_{G}(a, D) \leq a_{0}$ for all $D \subseteq E$, and equality holds if and only if there exist $x \in L_{G}(D)$ and $y \in R_{G}(D)$ such that $a^{T}(x+y)=a_{q}$. In general, establishing the load and the residual of an edge set $D \subseteq E$ with respect to a valid inequality $a^{T} x \leq a_{0}$ can be quite hard. However, for a specific $D$ it can be relatively easy to find $x \in L_{G}(D)$ and $y \in R_{G}(D)$ with $a^{T}(x+y)=a_{0}$, in which case $\operatorname{load}_{G}(a, D)=a^{T} x$ and $\operatorname{res}_{G}(a, D)=a^{T} y$. We need one more definition to formulate our second lifting theorem.

Definition 2.12 Let $G=(V, E)$ be a graph, let $a^{T} x \leq a_{0}$ be a valid inequality for $P(G)$, and let $E_{1}, \ldots, E_{k} \subseteq E$. Then the intersection graph $G\left(E_{1}, \ldots, E_{k} ; a\right)=(U, F)$ is defined by $U=\{1, \ldots, k\}$, and $\{i, j\} \in F$ if and only if there is an edge $e \in E_{i} \cap E_{j}$ with $a_{e} \neq 0$.

Theorem 2.13 (Patching) Given are a graph $G=(V, E)$ and a valid inequality $a^{T} x \leq a_{0}$ for $P(G)$. Let $E_{1}, \ldots, E_{k} \subseteq E(k \geq 2)$ be proper subsets of $E$ such that

(i) $E=\bigcup_{l=1}^{k} E_{l}$,

(ii) $\operatorname{load}_{G}\left(a, E_{l}\right)>0$ and $\bar{a}_{E_{l}}^{T} \widetilde{x}_{E_{l}} \leq \operatorname{load}_{G}\left(a, E_{l}\right)$ defines a facet of $P\left(V, E_{l}\right)$ for each $l=1, \ldots, k$,

(iii) $\operatorname{load}_{G}\left(a, E_{l}\right)+\operatorname{res}_{G}\left(a, E_{l}\right)=a_{0}$ for all $l=1, \ldots, k$,

(iv) the intersection graph $G\left(E_{1}, \ldots, E_{k} ; a\right)$ is connected.

Then the inequality $a^{T} x \leq a_{0}$ defines a nontrivial facet of $P(G)$.

Proof The face $F_{a}:=\left\{x \in P(G) \mid a^{T} x=a_{0}\right\}$ is contained in the facet $F_{a d}$ of $P(G)$ defined by some valid inequality $d^{T} x \leq d_{0}$. First, we show that there exists a $\beta_{l}>0$ such that $d_{i j}=\beta_{l} \cdot a_{i j}$ for all $\{i, j\} \in E_{l}(l=1, \ldots, k)$. For each $l=1, \ldots, k$ choose a maximal set $M_{l}$ of affinely independent integral points $x=x_{E_{l}} \in P(G)$ satisfying $\bar{x}_{E_{l}} \in P\left(V, E_{l}\right)$ and $a^{T} x=\operatorname{load}_{G}\left(a, E_{l}\right)$. According 
to condition (iii) we can find a vector $y^{l} \in R_{G}\left(E_{l}\right)$ with $a^{T} y^{l}=\operatorname{res}_{G}\left(a, E_{l}\right)$ and $x+y^{l} \in P\left(V, E_{l}\right)$ for all $x \in M_{l}$, thus yielding

$$
\begin{aligned}
a^{T}\left(x+y^{l}\right) & =a^{T} x+a^{T} y^{l} \\
& =\operatorname{load}_{G}\left(a, E_{l}\right)+\operatorname{res}_{G}\left(a, E_{l}\right) \\
& =a_{0} \quad \forall x \in M_{l} \quad(l=1, \ldots, k)
\end{aligned}
$$

Since none of the facets described in condition (ii) contains the zero vector, each set $M_{l}$ is linearly independent, and hence $\left\{x+y^{l} \mid x \in M_{l}\right\}$ is also linearly independent. It follows that for each $l=1, \ldots, k$ there exists a $\beta_{l}>0$ such that $d_{E_{l}}=\beta_{l} \cdot a_{E_{i}}$.

It remains to show that $\beta_{l}=\beta_{m}$ for all $l, m=1, \ldots, k$. Let $l, m \in\{1, \ldots, k\}$ be such that the corresponding vertices in the intersection graph $G\left(E_{1}, \ldots, E_{k} ; a\right)$ are adjacent. Then there exists $\{i, j\} \in E_{l} \cap E_{m}$ with $a_{i j} \neq 0$ and $\beta_{l} \cdot a_{i j}=$ $\beta_{m} \cdot a_{i j}$, hence $\beta_{l}=\beta_{m}$. Since the intersection graph is connected it follows that $\beta_{1}=\ldots=\beta_{k}$.

Notice that, given the edge sets $E_{1}, \ldots, E_{k}$, and provided that the load and residual of each edge set is known, it is easy to check whether conditions (i) (iv) of Theorem 2.13 are fulfilled.

Before deriving a corollary from Theorem 2.13, let us first illustrate its use.

Example 2.14 Let $K_{5}$ be the complete graph on five vertices. The 2-chorded cycle inequality

$$
x_{12}+x_{23}+x_{34}+x_{45}+x_{51}-x_{13}-x_{24}-x_{35}-x_{41}-x_{52} \leq 2
$$

can be covered by triangle inequalities in the sense of Theorem 2.13: the five sets

$$
\left.E_{l}=E(\{l, l+1, l+2\}) \quad \text { (numbers modulo } 5,1 \leq l \leq 5\right)
$$

meet all requirements. 
In fact, inequality (2.5) defines a facet for any polytope corresponding to a graph containing $K_{5}$ as a subgraph, which is an immediate consequence of the following corollary.

Corollary 2.15 Given are a graph $G=(V, E)$ and a valid inequality $a^{T} x \leq$ $a_{0}$ for $P(G)$. Let $U$ be a proper subset of $V$ such that $E(U)$ includes the support of $a^{T} x \leq a_{0}$ and the following two conditions hold:

(i) $\bar{a}_{E(U)}^{T} \bar{x}_{E(U)} \leq a_{0}$ defines a facet of $P(U, E(U))$,

(ii) For each $u \in U$ incident to an edge between $U$ and $V \backslash U$ there exists some edge $\{i, j\} \in E(U \backslash\{u\})$ with

$$
\operatorname{load}_{G}(a, E(U \backslash\{u, i, j\}))=a_{0}-a_{i j}<a_{0}
$$

Then $a^{T} x \leq a_{0}$ defines a facet of $P(G)$.

Proof For each vertex $u \in U$ choose an edge $e(u)$ as described in (ii). Put

$$
E_{u}:=E(\{u\}, V \backslash U) \cup E(V \backslash U) \cup\{e(u)\}
$$

Then the sets $E_{u}(u \in U)$ together with $E(U)$ meet the requirements of Theorem 2.13.

Notice that given a complete graph on $2 k+1$ vertices $(k \geq 3)$ and a 2 -chorded cycle inequality with right-hand side $k$, the construction shown in Example 2.14 fails to cover all edges of $G$ and hence Theorem 2.13 (or Corollary 2.15) alone does not imply facetness of these inequalities for polytopes corresponding to complete graphs (or graphs containing complete subgraphs). More generally, invoking Theorem 2.13 for proving that a given inequality is facet defining for the polytope $P(V, E)$ would sometimes only confirm that the truncated inequality is facet defining for $P(V, D)$ where $D$ includes the support but is a proper subset of $E$. In such circumstances a straightforward construction may turn the base of $\mathbb{R}^{|D|}$ provided by Theorem 2.13 into an expanded base of $\mathbb{R}^{|E|}$ that resides within the face in question, thus proving facetness: 
Lemma 2.16 Given are a graph $G=(V, E)$ and an inequality $a^{T} x \leq a_{0}$ valid for $P(G)$. Let $D$ be a proper subset of $E$ such that $\bar{a}_{D}^{T} \bar{x}_{D} \leq a_{0}$ defines a nontrivial facet of $P(V, D)$. Let $F_{1}, \ldots, F_{k}$ be a covering of $E \backslash D$ such that for each $i=1, \ldots, k$ there exist $\left|F_{i}\right|$ solutions $x$ of $a^{T} x=a_{0}$ on $D \cup\left(\bigcup_{i=1}^{k} F_{i}\right)$ for which the corresponding truncated vectors $\bar{x}_{F_{i}}$ are linearly independent. Then $a^{T} x \leq a_{0}$ defines a facet of $P(G)$.

\subsection{General 2-partition inequalities}

This section serves as an illustration of our lifting theorems described in the previous two sections. Proving that a given inequality $a^{T} x \leq a_{0}$ defines a facet of $P(G)$ amounts to two things: first, one has to show that the inequality is valid for $P(G)$, and second one must, directly or indirectly, show that the face $F_{a}:=\left\{x \in P(G) \| a^{T} x=a_{0}\right\}$ contains $\operatorname{dim}(P(G))$ affinely independent vectors. The following lemma provides a short and generally applicable argument showing validity of any inequality with coefficients in $\{0, \pm 1\}$ without a triangle in the positive support, where $\nu(G)$ denotes the maximal cardinality of a matching in $G$.

Lemma 2.17 Let $K_{n}=\left(V_{n}, E_{n}\right)$ be a complete graph, let $G=(V, E)$ be a connected, triangle-free subgraph of $K_{n}$, and let $E^{\prime} \subseteq E_{n}$ be the set of 2-chords of $G$. Then the inequality

$$
x(E)-x\left(E^{\prime}\right) \leq \nu(G)
$$

is valid for $P\left(K_{n}\right)$.

Proof Let $A \subseteq E_{n}$ be a clique in $K_{n}$, and let $G^{t}=(V(A), A \cap E)$. Then it is sufficient to show that

$$
|A \cap E|-\left|A \cap E^{\prime}\right| \leq \nu\left(G^{\prime}\right)
$$

Let $M$ be a matching in $G^{\prime}$ of maximal cardinality. Construct the mapping $\varphi:(A \cap E) \backslash M \rightarrow A \cap E^{\prime}$ as follows. Take $\{i, j\} \in(A \cap E) \backslash M$, then at least one of the vertices $i$ and $j$ is covered by $M$. If only one vertex, say $i$, is covered 
by $M$ (say by $\{i, k\} \in M$ ), map edge $\{i, j\}$ onto $\{j, k\}$, which is in $A \cap E^{\prime}$. If both $i$ and $j$ are covered by $M$ (say $\{i, k\},\{j, l\} \in M$ ), then map $\{i, j\}$ again onto $\{j, k\}$, and if $\{k, l\}$ is also in $(A \cap E) \backslash M$, then map this edge onto $\{i, l\}$. Obviously $\varphi$ is injective, hence $|(A \cap E) \backslash M| \leq\left|A \cap E^{\prime}\right|$, and thus

$$
|A \cap E|-\left|A \cap E^{\prime}\right| \leq|M|=\nu\left(G^{\prime}\right)
$$

Notice that if $a^{T} x \leq a_{0}$ is valid for $P\left(K_{n}\right)$, then it is also valid for $P(G)$ where $G$ is a subgraph of $K_{n}$, since any clique partition of $G$ can also be seen as a clique partition of $K_{n}$. It follows directly from Lemma 2.17 that the triangie inequalities, the 2-partition inequalities, and the 2-chorded cycle inequalities are valid for the clique partitioning polytope of arbitrary graphs. However, it is not immediate when such an inequality is facet defining. Consider for instance the $(S, T)$-inequality with $S=\{1\}$ and $T=\{2,3\}$ in Example 2.7a, which does not induce a facet. We will now characterize when a 2-partition inequality defines a facet under the additional hypothesis that $E(S, T)$ has full size $|S| \cdot|T|$. This generalizes a result in [39].

Theorem 2.18 Given a graph $G=(V, E)$, let $S, T \subseteq V$ be disjoint subsets, such that $1 \leq|S| \leq|T|$ and $\{\{s, t\} \mid s \in S, t \in T\} \subseteq E$. Then the $(S, T)$ inequality

$$
x(E(S, T))-x(E(S))-x(E(T)) \leq|S|
$$

defines a facet of $P(G)$ if and only if $|S|<|T|$ and the following two conditions hold:

(i) if $|S|>1$, then $E(T) \neq \varnothing$,

(ii) every vertex of $G$ outside $S \cup T$ which is adjacent to some vertex in $S$ is also adjacent to some vertex in $T$.

Proof First we show that the conditions are necessary. If $|S|=|T|$, then every clique partition for which the characteristic vector belongs to the face $F$ supported by (2.7) consists of cliques that intersect $S$ and $T$ in equally sized subsets. Therefore all members of $F$ satisfy the additional equality $x(E(S))-$ $x(E(T))=0$, whence $F$ cannot be a facet. 
If $2 \leq|S|<|T|$ but $E(T)=\varnothing$, then every clique partition with incidence vector $x \in F$ must be a matching, so that again $x(E(S))-x(E(T))=0$ holds.

If there is some edge $e=\{s, v\} \in E$ with $s \in S, v \notin S \cup T$, and $\{v, t\} \notin E$ for all $t \in T$, then we may add $x_{e}$ to the left-hand side of (2.7) and still obtain an inequality valid for $P(G)$. Hence $F$ is not a facet.

Conversely, assume that all requirements of the theorem are met. For each triplet $s, t, u \in V$ with $s \in S$ and $t, u \in T$ we take the edge set $E(\{s, t, u\})$. These edge sets form a connected intersection graph with respect to (2.7) since $S$ is a singleton or $E(T)$ is nonempty (or both). From Theorem 2.13 we infer that the truncated inequality is facet defining for $P(V, E(S, T) \cup E(T))$. An application of Corollary 2.15 then shows that this is also true for the higher dimensional polytope $P(V, D)$ where

$$
D:=E(V \backslash T, T) \cup E(T) \cup E(V \backslash(S \cup T))=E \backslash(E(S, V \backslash T))
$$

Each edge $e \in E \backslash D$ is extended to a clique: if $e \in E(S)$ then the clique $E(e \cup f)$ is constructed where $f \in E(T)$ (which exists by condition (i)); else a triangle $E(e \cup\{t\})$ is constructed where $t \in T$ is chosen appropriately (this is possible by condition (ii)). Each of these cliques can be extended to a clique partition of $G$ that otherwise uses only single edges between $S$ and $T$ as cliques. These $|E \backslash D|$ clique partitions satisfy the conditions of Lemma 2.16. This ensures that the $(S, T)$-inequality is indeed facet defining for $P(G)$.

Theorem 2.13 can also be used to construct relatively short proofs that inequalities define facets, as is illustrated in the next example. This entails, as a particular instance, the fact that the inequalities considered by Grötschel and Wakabayashi [40] define facets of the clique partitioning polytope associated with complete graphs.

Example 2.19 Let $K_{n}=\left(V_{n}, E_{n}\right)$ be a complete graph on $n$ vertices, and let $S_{1}, \ldots, S_{k}$ be pairwise disjoint subsets of $V_{n}$ which are disjoint from subsets $T_{1}, \ldots, T_{k} \subseteq V(k \geq 2)$, satisfying the following additional conditions. For any pairwise intersecting sets selected from $T_{1}, \ldots, T_{k}$, all pairwise intersections are equal and contain at least two vertices. The intersection graph of the 
sets $T_{1}, \ldots, T_{k}$ is a block graph, that is, a grapl in which every maximal 2-connected subgraph is complete. Moreover,

$$
1 \leq\left|S_{i}\right| \leq\left|T_{i}\right|-\max _{\substack{j=1, \ldots, k \\ j \neq i}}\left|T_{i} \cap T_{j}\right| \quad \forall i=1, \ldots, k
$$

Then the general 2-partition inequality

$$
\begin{gathered}
\sum_{i=1}^{k} x\left(E\left(S_{i}, T_{i}\right)\right)-\sum_{i=1}^{k} x\left(E\left(S_{i}\right)\right)-x\left(\bigcup_{i=1}^{k} E\left(T_{i}\right)\right) \\
-\sum_{\substack{1 \leq i<j \leq k \\
T_{i} \cap T_{j} \neq \varnothing}} x\left(E\left(S_{i}, S_{j}\right)\right) \leq \sum_{i=1}^{k}\left|S_{i}\right|
\end{gathered}
$$

is walid and facet defining for $P\left(K_{n}\right)$.

Notice that the inequalities considered by Grötschel and Wakabayashi [40] are simply inequalities (2.8) under the constraint that the intersection graph of the sets $T_{1}, \ldots, T_{k}$ is a path or a clique.

Validity of (2.8) immediately follows from Lemma 2.17. Let us now present a proof of 'facetness' based on Theorem 2.13 and Lemma 2.16. Put $E_{i}:=$ $E\left(S_{i} \cup T_{i}\right)$ for $i=1, \ldots, k$. For each index $i$ the load of inequality (2.8) with respect to $E_{i}$ equals $\left|S_{i}\right|$ and the corresponding residual equals the sum of all $\left|S_{j}\right|$ with $j \neq i$. Hence all four requirements of Theorem 2.13 are met for the subgraph $(V, D)$ of $G$ where $D:=\bigcup_{i=1}^{k} E_{i}$. Therefore the truncation of (2.8) to the edge set $D$ defines a facet of $P(V, D)$.

Notice that the number of vertices in $T:=\bigcup_{i=1}^{k} T_{i}$ exceeds the size of $S:=$ $\bigcup_{i=1}^{k} S_{i}$ by at least 2. Furthermore, any matching in the graph $(V, D)$ comprising $|S|$ edges each joining a vertex in $S$ and a vertex in $T$ constitutes a clique partitioning of $G$ satisfying (2.8) at equality. We will refer to such a matching as an $S$-matching. It is easy to see that for every pair $\{u, v\} \subseteq T$ there is an $S$-matching not incident to $\{u, v\}$.

In order to fulfill the requirements of Lemma 2.16, we first exhibit for each edge $\{u, v\} \in E \backslash D$ a clique partitioning which includes $\{u, v\}$ as well as an appropriate $S$-matching $M_{u v}$, so that (2.8) is satisfied with equality. 
(1) Assume $\{u, v\} \subseteq T$. Then $\{u, v\} \mathbb{Z} T_{i}$ for all $i$. Pick any $S$-matching $M_{u v}$ not incident to $\{u, v\}$. Then $M_{w v} \cup\{\{u, v\}\}$ is a clique partitioning satisfying (2.8) at equality.

(2) Assume $u \in S$ and $v \in T$, say $u \in S_{i}$ and $v \in T_{j}$ (where $v \notin T_{i}$ ). For any choser $t_{u} \in T_{i}$ there exists an $S$-matching $M_{u w}$ containing $\left\{u, t_{u}\right\}$ but not incident to $v$. Then $M_{u v} \cup\left\{\{u, v\},\left\{t_{u}, v\right\}\right\}$ is a clique partitioning satisfying (2.8) at equality.

(3) Assume $\{u, v\} \subseteq S$ say $u \in S_{i}$ and $v \in S_{j}$ (for $j \neq i$ ). If $T_{i} \cap T_{j} \neq \varnothing$, select two distinct vertices $t_{u}$ and $t_{v}$ in $T_{i} \cap T_{j}$; else pick $t_{u} \in T_{i}$ and $t_{v} \in T_{j}$ arbitrarily. In either case, we can choose an $S$-matching $M_{u v}$ containing $\left\{t_{u}, u\right\}$ and $\left\{t_{v}, v\right\}$. Then $M_{u v} \cup\left\{\{u, v\},\left\{t_{u}, t_{v}\right\},\left\{u, t_{v}\right\},\left\{v, t_{u}\right\}\right\}$ is a clique partitioning satisfying $(2.8)$ at equality.

Now let $F_{1}, F_{2}, F_{3}$ be the sets of clique partitionings constructed in the preceding steps (1), (2), (3). Clearly, $F_{1} \cup F_{2} \cup F_{3}$ covers $E \backslash D$. Since every clique partitioning in $F_{1}, F_{2}, F_{3}$ contains exactly one edge of $E \backslash D$ intersected with $E(T), E(S, T)$, and $E(S)$ respectively, the conditions in Lemma 2.16 are met for the sets $F_{1}, F_{2}, F_{3}$. We conclude that (2.8) defines a facet of $P(G)$.

\section{Acknowledgements}

This chapter is based on joint work with Hans-Jürgen Bandelt, Maarten Oosten, and Frits Spieksma. 


\section{Chapter 3}

\section{New facets and patching}

\subsection{Introduction}

The results described in this chapter are of two kinds. The next section describes a number of new classes of facets for the clique partitioning polytope of complete graphs. Four of the new facets have all coefficients in $\{0, \pm 1\}$, but the other class contains facets with arbitrary coefficients. Section 3.3 describes a technique, called patching, to construct new facets from known ones. Patching takes as input two or more small facets, and glues them together to a new big facet, where small and big refer to the support of the various lacet defining inequalities. The patching procedure given here is different from the one given in Chapter 2, which can only be applied if the intersection graph of the small facets is connected, i.e. the small facets must overlap. In this chapter we call this procedure intersection patching, in contrast to the case were the small facets do not overlap, called disjoint patching, which is described in Section 3.4. The results of this chapter enable us to characterize all facets of $P\left(K_{n}\right)$ with right-hand side 1 or 2 , which is done is Section 3.5 .

Before describing new facets of the clique partitioning polytope, let us first give an overview of known valid and facet defining inequalities. Grötschel and Wakabayashi [39] present the following three classes of facets of $P\left(K_{n}\right)$. 
Theorem 3.1 [39] Let $S, T \subseteq V_{n}$ be two nonempty disjoint subsets of $V_{n}$. The 2-partition inequality

$$
x\left(E_{n}(S, T)\right)-x\left(E_{n}(S)\right)-x\left(E_{n}(T)\right) \leq \min \{|S|,|T|\}
$$

is valid for $P\left(K_{n}\right)$. It defines a facet if and only if $|S| \neq|T| . \square$

Theorem 3.2 [39] Let $C \subseteq E_{n}$ be a cycle of length at least 5 in $K_{n}$, and let $\bar{C}=\mathrm{Ch}_{2}(C)$. The 2 -chorded cycle inequality

$$
x(C)-x(\bar{C}) \leq\left\lfloor\frac{1}{2}|C|\right\rfloor
$$

is valid for $P\left(K_{n}\right)$. It defines a facet if and only if $|C|$ is odd.

Theorem 3.3 [39] Let $P=\left\{\left\{u_{i}, u_{i+1}\right\} \mid i=1, \ldots, k\right\}$ be a path of length $k$, let $\widetilde{P}=\mathrm{Ch}_{2}(P)$, and let $z \in V_{n}$ be a vertex not incident to $P$. Define

$$
\begin{aligned}
& R:=\left\{\left\{z, u_{i}\right\} \mid i=1, \ldots, k, i \text { even }\right\} \\
& \bar{R}:=\left\{\left\{z, u_{i}\right\} \mid i=1, \ldots, k, i \text { odd }\right\}
\end{aligned}
$$

The 2-chorded path inequality

$$
x(P \cup R)-x(\bar{P} \cup \bar{R}) \leq\left\lfloor\frac{1}{2}(|P|+1)\right\rfloor
$$

is valid for $P\left(K_{n}\right)$. It defines a facet if and only if $|P|$ is even.

Notice that if $P=\left\{\left\{u_{1}, u_{2}\right\},\left\{u_{2}, u_{3}\right\}\right\}$ and $z \in V_{n} \backslash V_{n}(P)$ the corresponding 2-chorded path inequality is nothing but the $(S, T)$-inequality with $S=\left\{u_{2}\right\}$ and $T=\left\{z, u_{1}, u_{3}\right\}$. If $P$ is an even cycle in $K_{n}$, i.e. $|P|$ is even and the endpoints of $P$ coincide, then (3.3) is also valid and facet defining for $P\left(K_{n}\right)$, and it is called a 2-chorded wheel inequality. The classes of facets described in [40] form a subset of the general 2-partition inequalities (2.8) given in the previous chapter.

Facets of the multicut polytope $M C_{n}^{\leq}\left(K_{n}\right)$ can directly be transformed to facets of $P\left(K_{n}\right)$. Deza and Laurent [24] introduce the class of clique-web inequalities for the multicut polytopes, which easily translates to a class of facets for $P\left(K_{n}\right)$. Let us first give definitions of a web and an antiweb of $K_{n}$. 
Definition 3.4 Let $K_{n}=\left(V_{n}, E_{n}\right)$ be a complete graph. The antiweb $A W_{p}^{r}$ with parameters $p \geq 1$ and $r \geq 0$ is the subgraph of $K_{n}$ with vertex set $\left\{v_{1}, \ldots, v_{p}\right\} \subseteq V_{n}$ and edges $\left\{v_{i}, v_{i+k}\right\}$ for all $i=1, \ldots, p$ and $k=1, \ldots, q^{n}$ (indices modulo $p$ ). The web $W_{p}^{r}$ is the complement graph of the antiweb $A W_{p}^{r}$.

The antiweb $A W_{p}^{r}$ is a $p$-cycle together with all $k$-chords for $2 \leq k \leq r$. The web $W_{p}^{r}$ with $p=2 r+3$ is just a cycle of length $2 r+3$. To shorten notation we simply use $W_{p}^{r}$ or $A W_{p}^{r}$ to denote the edge set of an (anti)web, since the edge set implicitly defines the node set. The clique-web inequalities for the clique partitioning polytope are given by the following theorem.

Theorem 3.5 [24] Let $K_{n}=\left(V_{n}, E_{n}\right)$ be a complete graph, let $W_{p}^{r}(p \geq 1$, $r \geq 0)$ be a web in $K_{n}$ with vertex set $W$, and let $U \subseteq V_{n} \backslash W$ with $|U|=q \geq 1$ such that $p-q \geq 2 r+1$. The clique-web inequality

$$
x(E(U, W))-x\left(W_{p}^{r}\right)-x(E(U)) \leq q(r+1)
$$

is valid for $P\left(K_{n}\right)$. It defines a facet if $p-q \geq 2 r+1$ and $q \geq 2$ if $p-q=2 r+1$.

Notice that the 2-partition inequalities occur as clique-web inequalities with $r=0$. The clique-web inequality with $q=1$ and $p=2 r+3$ can alternatively be formulated as follows.

Theorem 3.6 Let $C \subseteq E_{n}$ be a cycle of length at least 3 in $K_{n}$, and let $z \in V_{n}$ be a vertex not incident to $C$. The wheel inequality

$$
x\left(E_{n}\left(\{z\}, V_{n}(C)\right)\right)-x(C) \leq\left\lfloor\frac{1}{2}|C|\right\rfloor
$$

is valid for $P\left(K_{n}\right)$. It defines a facet if and only if $|C|$ is odd.

The wheel inequalities were introduced by Chopra and Rao [14]. They prove that these inequalities define facets for both the $k$-partition polytope and the clique partitioning polytope. 
If $q=2$ and $p=2 r+3$ then (3.4) can also be defined as follows. Let $C=W_{2 r+3}^{r} \subseteq E_{n}$ be an odd cycle in $K_{n}$, and let $U \subseteq V_{n}$ be a set of two vertices not incident to $C$. The clique-web inequality induced by $W_{2 r+3}^{r}$ and $U$ is equivalent to

$$
x\left(E_{n}\left(U, V_{n}(C)\right)\right)-x\left(E_{n}(U)\right)-x(C) \leq|C|-1
$$

We refer to (3.6) as bicycle wheel inequalities.

\subsection{New facets}

In this section we describe two different generalizations of 2-partition inequalities with right-hand side 1 , and we give sufficient conditions for these inequalities to be facet defining. We also describe generalizations of the 2-chorded cycle and the 2-chorded path and wheel inequalities.

\subsubsection{Claw inequalities}

Theorem 3.7 Let $K_{n}=\left(V_{n}, E_{n}\right)$ be a complete graph, $s \in V_{n}, T \subseteq V_{n} \backslash\{s\}$, and let $c \geq 2$ be an integer. The claw inequality

$$
c \cdot x\left(E_{n}(\{s\}, T)\right)-x\left(E_{n}(T)\right) \leq\left(\begin{array}{c}
c+1 \\
2
\end{array}\right)
$$

is valid for $P\left(K_{n}\right)$. It defines a facet if and only if $|T| \geq c+2$.

The support of a claw inequality is shown in Figure 3.1. Notice that for $c=1$ a claw inequality reduces to a 2-partition inequality induced by $\{s\}$ and $T$. In order to prove Theorem 3.7 we need the following lemma.

Lemma 3.8 Let $\mathbb{R}_{i} \in \mathbb{N}$, and let $K_{n}=\left(V_{n}, E_{n}\right)$ be a complete graph with $V_{n}=\{s\} \cup T,|T|=k+2$. For each $T^{\prime} \subseteq T, k \leq\left|T^{\prime}\right| \leq k+1$, let $a_{T^{\prime}}$ be the characteristic vector of $E_{n}\left(\{s\} \cup T^{\prime}\right)$. The matrix $A^{k}$ constructed from all these characteristic vectors is nonsingular. 


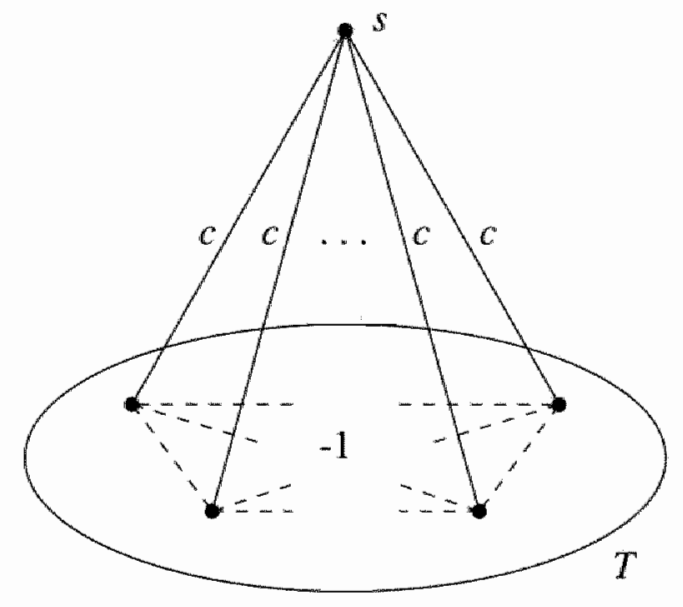

Figure 3.1: Support of a claw inequality.

Proof The graph $K_{n}$ contains $\left(\begin{array}{c}k+3 \\ 2\end{array}\right)$ edges, and the matrix $A^{k}$ contains

$$
\left(\begin{array}{c}
k+2 \\
k
\end{array}\right)+\left(\begin{array}{c}
k+2 \\
k+1
\end{array}\right)=\left(\begin{array}{c}
k+2 \\
2
\end{array}\right)+\left(\begin{array}{c}
k+2 \\
1
\end{array}\right)=\left(\begin{array}{c}
k+3 \\
2
\end{array}\right)
$$

rows, so $A^{k}$ is a square matrix. By means of row and column permutations, $A^{k}$ can be written as

$$
A^{k}=\left[\begin{array}{c|c}
A_{11}^{k} & A_{12}^{k} \\
\hline A_{21}^{k} & A_{22}^{k}
\end{array}\right]
$$

where the rows $\left[A_{11}^{k} \mid A_{12}^{k}\right]$ correspond to $\left|T^{\prime}\right|=k$, and the rows $\left[A_{21}^{k} \mid A_{22}^{k}\right]$ correspond to $\left|T^{\prime}\right|=k+1$. Furthermore, the columns

$$
\left[\frac{A_{1 \rrbracket}^{k}}{A_{2 \Downarrow}^{k}}\right], \quad\left[\frac{A_{12}^{k}}{A_{22}^{k}}\right]
$$


correspond to $E_{n}(\{s\}, T)$ and $E_{n}(T)$ respectively. As one can verify, for all $k \geq 1$, the following holds.

\begin{tabular}{|c|c|c|c|c|}
\hline & \# rows & \# columns & row sum & column sum \\
\hline$A_{11}$ & $\left(\begin{array}{c}k+2 \\
2\end{array}\right)$ & $k+2$ & $k$ & $\left(\begin{array}{c}k+1 \\
2\end{array}\right)$ \\
$A_{12}$ & $\left(\begin{array}{c}k+2 \\
2\end{array}\right)$ & $\left(\begin{array}{c}k+2 \\
2\end{array}\right)$ & $\left(\begin{array}{c}k \\
2\end{array}\right)$ & $\left(\begin{array}{c}k \\
2\end{array}\right)$ \\
$A_{21}$ & $k+2$ & $k+2$ & $k+1$ & $k+1$ \\
$A_{22}$ & $k+2$ & $\left(\begin{array}{c}k+2 \\
2\end{array}\right)$ & $\left(\begin{array}{c}k+1 \\
2\end{array}\right)$ & $k$ \\
\hline
\end{tabular}

We use induction to prove that $A^{k}$ is nonsingular. Clearly, $A^{1}$ can be written as follows.

$$
A^{1}=\left[\begin{array}{lll|lll}
1 & 0 & 0 & 0 & 0 & 0 \\
0 & 1 & 0 & 0 & 0 & 0 \\
0 & 0 & 1 & 0 & 0 & 0 \\
\hline 1 & 1 & 0 & 1 & 0 & 0 \\
1 & 0 & 1 & 0 & 1 & 0 \\
0 & 1 & 1 & 0 & 0 & 1
\end{array}\right]
$$

Obviously, $A^{1}$ is nonsingular, since $A^{1}$ is a triangular matrix. Notice also that by means of row and column permutations $A_{21}^{k}$ can be written as $A_{21}^{k}=J-I$, where $J$ is a matrix with all entries equal to 1 , and hence $A_{21}^{k}$ is nonsingular for all $k \geq 1$.

Now, assuming that the lemma holds for some $k \in \mathbb{N}$, we will show that it also holds for $k+1$. For $A^{k+1}$ we have

$$
\begin{aligned}
& A_{11}^{k+1}=\left[\begin{array}{l|l}
A_{11}^{k} & 1 \\
\hline A_{21}^{k} & \mathbf{0}
\end{array}\right], \quad A_{12}^{k+1}=\left[\begin{array}{c|c}
A_{12}^{k} & A_{11}^{k} \\
\hline A_{22}^{k} & \mathbf{0}
\end{array}\right], \\
& A_{21}^{k+1}=\left[\begin{array}{c|c}
A_{21}^{k} & \mathbf{1} \\
\hline \mathbf{1} & \mathbf{0}
\end{array}\right], \quad A_{22}^{k+1}=\left[\begin{array}{c|c}
A_{22}^{k} & A_{21}^{k} \\
\hline \mathbf{1} & \mathbf{0}
\end{array}\right],
\end{aligned}
$$


By Gaussian elimination $A^{k+1}$ can be transformed into

$$
\left[\begin{array}{c|c|c|c}
A_{11}^{k} & \vdots & A_{12}^{k} & A_{11}^{k} \\
& 1 & & \\
\hline & 0 & & \\
A_{21}^{k} & \vdots & A_{22}^{k} & 0 \\
& 0 & & \\
\hline 0 & \vdots & 0 & A_{21}^{k} \\
\hline 1 \cdots 1 & 0 & 1 \cdots 1 & 0 \cdots 0
\end{array}\right]
$$

It is obvious that all rows of $A^{k+1}$ but the last one are linearly independent, since both $A^{k}$ and $A_{21}^{k}$ are nonsingular. Let $p=\left(\begin{array}{c}k+3 \\ 2\end{array}\right)$ and $q=k+2$. If the last row of $A^{k+1}$ is a linear combination of the other rows, then there exist numbers $\alpha_{1}, \ldots, \alpha_{p}$ such that

$$
\left(\alpha_{1}, \ldots, \alpha_{p}\right) A^{k}=(1, \ldots, 1)
$$

Since $A^{k}$ is nonsingular, this equation has only one solution. With the column sums of $A^{k}$ in mind, it is easy to check that

$$
\alpha_{1}=\ldots=\alpha_{p-q}=-\frac{2}{k(k+1)}, \quad \alpha_{p-q+1}=\ldots=\alpha_{p}=\frac{2}{k+1}
$$

Furthermore, there have to exist numbers $\beta_{1}, \ldots, \beta_{q}$, such that:

$$
\left(\alpha_{1}, \ldots, \alpha_{p-q}\right) A_{11}^{k}+\left(\beta_{1}, \ldots, \beta_{q}\right) A_{21}^{k}=(0, \ldots, 0)
$$

Since $\alpha_{1}, \ldots, \alpha_{p-q}$ are already fixed, and since $A_{21}^{k}$ is nonsingular, this equation has only one solution. It is easy to check that $\beta_{1}=\ldots=\beta_{q}=\frac{1}{k+1}$. Finally, also the following equation must be satisfied.

$$
\alpha_{1}+\ldots+\alpha_{p-q}+\beta_{1}+\ldots+\beta_{q}=0
$$


Substituting the known values for $\alpha_{1}, \ldots, \alpha_{p-q}$ and $\beta_{1}, \ldots, \beta_{q}$ shows that this equation is not satisfied. Hence, the matrix $A^{k+1}$ is nonsingular.

\section{Proof of Theorem 3.7:}

First we show that (3.7) is valid, and that it does not define a facet if $|T| \leq c+1$. Let $A \subseteq E_{n}$ be an arbitrary clique partition, and let $A^{\prime} \subseteq A$ be the clique incident to $s$. The left-hand side of (3.7) is maximal if $c \leq\left|V_{n}\left(A^{\prime}\right) \cap T\right| \leq c+1$, and in that case the left-hand side and the right-hand side of (3.7) are equal. Hence the claw inequality is valid (see also Müller and Schulz [55]).

If $|T| \leq c-1$, then there is no clique partition satisfying (3.7) at equality, so it defines an empty face. If $|T|=c$ then all clique partitions satisfying (3.7) at equality contain the clique $E_{n}(\{s\} \cup T)$. Hence the face induced by $(3.7)$ is contained in the intersection of the hyperplanes

$$
\begin{array}{ll}
x_{s t}=1 & \forall t \in T \\
x_{t u}=1 & \forall t, u \in T, t \neq u
\end{array}
$$

and therefore it does not define a facet. If $|T|=c+1$, then (3.7) is nothing but the sum of the $\left(\begin{array}{c}c+1 \\ 2\end{array}\right)$ triangle inequalities

$$
x_{s u}+x_{s v}-x_{u v} \leq 1 \quad \forall u, v \in T, u \neq v
$$

and thus it is not facet defining.

It remains to prove that inequalities (3.7) define facets if $|T| \geq c+2$. Denote such an inequality by $a^{T} x \leq a_{00}$, and let

$$
F_{a}:=\left\{x \in P\left(K_{n}\right) \mid a^{T} x=a_{0}\right\} \subseteq F_{b}:=\left\{x \in P\left(K_{n}\right) \mid b^{T} x=b_{0}\right\}
$$

where $F_{b}$ is a facet. In order to prove that $F_{a}$ is a facet if $c \geq 2$, we show that any facet that contains $F_{a}$ is identical to $F_{a}$. Since any proper face is either a facet or contained in one, this is sufficient to prove the theorem.

Consicler all clique partitions $M_{T^{\prime}}:=E_{n}\left(\{s\} \cup T^{\prime}\right)$ with $T^{\prime} \subseteq T$ and $c \leq\left|T^{\prime}\right| \leq$ $c+1$. Clearly, $\chi^{M_{T^{\prime}}} \in F_{a} \subseteq F_{b}$. According to Lemma 3.8 all characteristic vectors $\chi^{M_{7^{\prime \prime}}}$ are linearly independent, so we can conclude that

$$
b_{i j}=\frac{b_{0}}{a_{0}} \cdot a_{i j} \quad \forall\{i, j\} \in E_{n}(\{s\} \cup T)
$$


It remains to prove that $b_{i j}=0$ for all other edges $\{i, j\} \in E_{n}$. It is obvious that $b_{i j}=0$ if $\{i, j\}$ is not incident to the support of $(3.7)$, hence

$$
b_{i j}=0 \quad \forall i, j \in V_{n} \backslash(\{s\} \cup T), i \neq j
$$

Let $i \in V_{n} \backslash(\{s\} \cup T), t \in T, T^{\prime} \subseteq T \backslash\{t\},\left|T^{\prime \prime}\right|=c$, and let $M_{1}:=E_{n}\left(\{s\} \cup T^{\prime}\right)$, $M_{2}:=M_{1} \cup\{\{i, t\}\}$. Then $\chi^{M_{1}}, \chi^{M_{2}} \in F_{a} \subseteq F_{b}$, so

$$
0=b_{0}-b_{0}=b^{T} \chi^{M_{2}}-b^{T} \chi^{M_{1}}=b_{i t}
$$

Thus

$$
b_{i t}=0 \quad \forall i \in V_{n} \backslash(\{s\} \cup T), t \in T
$$

Let $M_{3}:=E_{n}\left(\{s, i\} \cup T^{\prime}\right)$, then $\chi^{M_{3}} \in F_{a} \subseteq F_{b}$, and

$$
0=b_{0}-b_{0}=b^{T} \chi^{M_{3}}-b^{T} \chi^{M_{1}}=b_{i s}+\sum_{t \in T^{\prime}} b_{i t}
$$

With (3.10) this implies that

$$
b_{i s}=0 \quad \forall i \in V_{n} \backslash(\{s\} \cup T)
$$

Equations (3.8), (3.9), (3.10), and (3.11) imply the theorem.

\subsubsection{Stable set inequalities}

The 2-partition inequalities can also be generalized in a way different from the one described in Theorem 3.7, as we show in the sequel.

Let $G=(V, E)$ be an arbitrary graph. A set $S \subseteq V$ is called a stable set or an independent set of $G$ if $E(S)=\varnothing$. The stability number of $G$ is the maximal cardinality of a stable set in $G$, and it is denoted by $\alpha(G)$. Moreover, $E$ is said to be minimal with respect to $\alpha(G)$ if removing any edge from $E$ increases the stability number. A set $S_{1}, \ldots, S_{k} \subseteq V$ is called a clique cover of $G$ if each 
set $S_{i}(i=1, \ldots, k)$ induces a clique in $G$ and $V=S_{1} \cup \ldots \cup S_{k}$. The clique cover number of $G$, i.e. the minimal cardinality of a clique cover, is denoted by $k(G)$.

Let $K_{n}=\left(V_{n}, E_{n}\right)$ be a complete graph and consider the following inequalities.

$$
x\left(E_{n l}(\{s\}, T)\right)-x(F) \leq \alpha(H)
$$

with $F \subseteq E_{n}, T=V_{n}(F) \neq V_{n}, s \in V_{n} \backslash T$, and $H=(T, F)$. We refer to inequalities (3.12) as stable set inequalities. Notice that if $H$ is complete the corresponding stable set inequality is nothing but a 2-partition inequality.

Theorem 3.9 The stable set inequalities (3.12) are valid for $P\left(K_{n}\right)$. The following conditions are necessary conditions for these inequalities to be facet defining.

(i) $F$ is minimal with respect to $\alpha(H)$,

(ii) $k(H)=\alpha(H)=1$ or $k(H)>\alpha(H) \geq 2$,

(iii) $H$ is connected,

(iv) For all $i \in T$ there is a stable set of size $\alpha(H)$ containing $i$,

(v) For all $i \in T$ there is a stable set of size $\alpha(H)$ not containing $i$.

Conditions (i)-(v), together with the following condition, are sufficient for $H$ to ensure that ( 3.12 ) is facet defining.

(vi) For all $i, j \in T,\{i, j\} \notin F$, there is a stable set $S \subseteq T$ of cardinality $\alpha(H)$ such that $S \cap\{i, j\}=\varnothing$.

Proof First, let us show that inequalities (3.12) are valid. Suppose that there exists a clique partition $A$ violating (3.12). Then $A$ has $\alpha(H)+q$ edges in $E_{n}(\{s\}, T)$ for some $q \geq 1$. Let $T^{\prime} \subseteq T$ be the set of vertices incident to the same clique of $A$ as vertex $s$, and let $H^{\prime}=\left(T^{\prime}, E_{n}\left(T^{\prime}\right) \cap F^{\prime}\right)$ be the graph induced by the vertices of $T^{\prime}$. Consider a stable set of maximum cardinality in $H^{\prime}$. Since $\alpha\left(H^{\prime}\right) \leq \alpha(H)$ and $\left|T^{\prime}\right|=\alpha(H)+q$, it follows that there are at least $q$ vertices in $T^{t}$ not in a maximal stable set. Thus the clique partition $A$ contains at least $q$ edges in $E_{n}\left(T^{\prime}\right) \cap F$. Hence, (3.12) is valid. 
Denote an inequality of type (3.12) by $a^{T} x \leq a_{0}$, and let $b^{T} x \leq b_{0}$ be a facet defining inequality such that

$$
F_{a}:=\left\{x \in P\left(K_{n}\right) \mid a^{T} x=a_{0}\right\} \subseteq F_{b}:=\left\{x \in P\left(K_{n}\right) \mid b^{T} x=b_{0}\right\}
$$

Now, we show that conditions (i) $-(v)$ are necessary conditions in order for $F_{a}$ to be a facet.

(i) Suppose that $F$ is not minimal with respect to $\alpha(H)$. Then there exists an edge $\{i, j\} \in F$ such that $H^{\prime}=(T, F \backslash\{i, j\})$ has stability number $\alpha\left(H^{\prime}\right)=$ $\alpha(H)$, and thus (3.12) is the sum of $x\left(E_{n}(\{s\}, T)\right)-x(F \backslash\{i, j\}) \leq \alpha\left(H^{\prime}\right)$ and $-x_{i j} \leq 0$.

(ii) Suppose that $k(H)=\alpha(H)=k \geq 2$, and let $W_{1}, \ldots, W_{k}$ be a partition of $T$, such that $E_{n}\left(W_{i}\right) \subseteq F$, i.e. $W_{1}, \ldots, W_{k}$ induce a clique cover of size $k$ of $H$. Define

$$
F^{\prime}:=F \backslash\left(\bigcup_{i=1}^{k} E_{n}\left(W_{i}\right)\right)
$$

Then (3.12) is the sum of the inequalities $x\left(E_{n}\left(\{s\}, W_{i}\right)\right)-x\left(E_{n}\left(W_{i}\right)\right) \leq 1$ $(i=1, \ldots, k)$ and $-x\left(F^{\prime \prime}\right) \leq 0$, and therefore (3.12) does not define a facet.

(iii) If $H$ is not connected, then there exists a partition of $T$ into $T_{1}$ and $T_{2}$, a partition of $F$ into $F_{1}=F \cap E_{n}\left(T_{1}\right)$ and $F_{2}=F \cap E_{n}\left(T_{2}\right)$, and two graphs $H_{i}=\left(T_{i}, F_{i}\right)(i=1,2)$ such that $(3.12)$ is the sum of $x\left(E_{n}\left(\{s\}, T_{i}\right)\right)-x\left(F_{i}\right) \leq$ $\alpha\left(H_{i}\right)$.

(iv)-(v) Let $A \subseteq E_{n}$ be a clique partition such that $a^{T} \chi^{A}=a_{0}$, and let $T^{\prime}=\{t \in T \mid\{s, t\} \in A\}$ and $H^{\prime}=\left(T^{\prime}, E_{n}\left(T^{\prime}\right) \cap F\right)$. Then $H^{\prime}$ contains a stable set of cardinality $\alpha(H)$. Let $i \in T$. If all stable sets of cardinality $\alpha(H)$ contain $i$ then $F_{a} \subseteq\left\{x \in P\left(K_{n}\right) \mid x_{s i}=1\right\}$, and hence $F_{a}$ is not a facet. Consequently, there exists a stable set of size $\alpha(H)$ not containing $i$. If no stable set of cardinality $\alpha(H)$ contains $i_{\text {, then }} F_{a} \subseteq\left\{x \in P\left(K_{n}\right) \mid x_{s i}=0\right\}$, and hence $F_{a}$ is not a facet.

Finally, we will show that conditions (i)-(vi) are sufficient for (3.12) to be facet defining. Then we have to prove that $b=\beta a$ for some $\beta \in \mathbb{R}$. In order to do so, let $i, j \in T,\{i, j\} \notin F$, and let $S$ be a stable set of size $\alpha(H)$ in $T$ 
containing neither $i$ nor $j$. Then the clique partition $A:=E_{n}(\{s\} \cup S)$ satisfies $a^{T} \chi^{A}=a_{0}$, and the clique partition $A \cup\{\{i, j\}\}$ is also in $F_{a}$. Comparing these clique partitions yields

$$
b_{i j}=0 \quad \forall i, j \in T,\{i, j\} \notin F
$$

Let $i, j \in T,\{i, j\} \in F$. Since $F$ is minimal with respect to $\alpha(H)$, we have that $\alpha\left(H^{\prime}\right)=\alpha(H)+1$ for $H^{\prime}=\{T, F \backslash\{\{i, j\}\})$. Let $S \subseteq T$ be a maximal stable set of $H^{\prime}$. Then $i, j \in S$, otherwise $H$ would also have a stable set of size $\alpha(H)+1$. Let $S^{\prime}:=S \backslash\{i, j\}$, then both $S^{\prime} \cup\{i\}$ and $S^{\prime} \cup\{j\}$ are maximal stable sets of $H$. So, for the clique partitions $A_{i}:=E_{n}\left(S^{\prime} \cup\{s, i\}\right)$ and $A_{j}:=E_{n}\left(S^{\prime} \cup\{s, j\}\right)$ we have that $\chi^{A_{i}}, \chi^{A_{j}} \in F_{a}$. By looking at the symmetric difference $A_{i} \triangle A_{j}$ it immediately follows that

$$
b_{s i}+\sum_{r \in S^{t}} b_{i r}=b_{s j}+\sum_{r \in S^{\prime}} b_{j r}
$$

Together with (3.13) it follows that $b_{s i}=b_{s j}=\beta$ for $i, j \in T,\{i, j\} \in F$. Since $H$ is connected, we have

$$
b_{s i}=\beta \quad \forall i \in T
$$

The clique partition $A_{i j}:=E_{n}(S \cup\{s\})$ also satisfies (3.12) at equality, and by looking at $A_{i} \triangle A_{i j}$ it follows that $b_{s i}+b_{i j}=0$, or equivalently that

$$
b_{i j}=-\beta \quad \forall i, j \in T,\{i, j\} \in F
$$

Let $i \in T, j \in V_{n u} \backslash(T \cup\{s\})$. From (iv) it follows that there is a stable set $S$ with cardinality $\alpha(H)$ in $T$ not containing $i$. Since the clique partitions $A:=E_{n}(S \cup\{s\})$ and $A^{\prime}:=A \cup\{\{i, j\}\}$ are both in $F_{a}$, it follows that

$$
b_{i j}=0 \quad \forall i \in T, j \in V_{n} \backslash(T \cup\{s\})
$$

Now it is easy to see that also $b_{s j}=0$ for all $j \in V_{n} \backslash(T \cup\{s\})$. Together with (3.13)-(3.16) this implies that $b=\beta a$. 
Remark 3.10 The stable set inequalities generalize the so-called wheel inequalities found by Chopra and Rao [14] (the wheel inequalities occur when $H=(T, F)$ is an odd cycle). Figure 3.2 shows two graphs which both satisfy conditions (i) - (vi), and which are therefore facet inducing graphs for the claw inequalities.
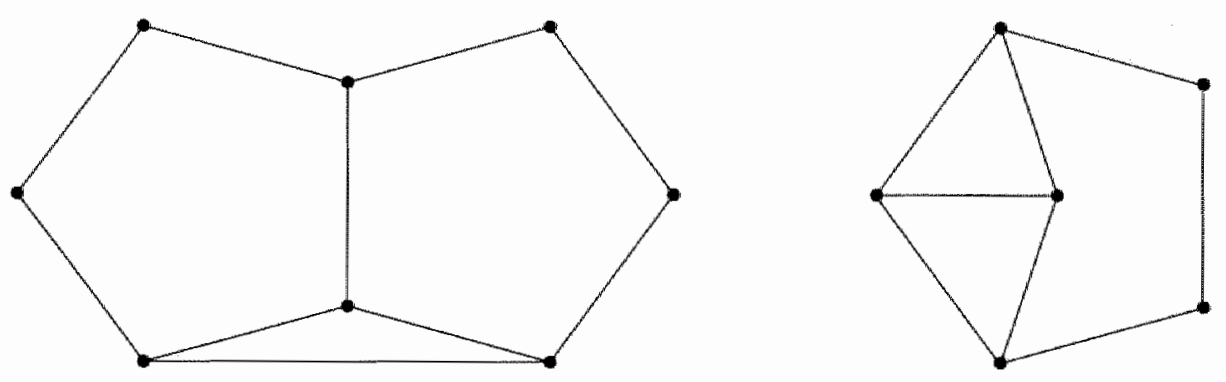

Figure 3.2: Facet inducing graphs for inequality (3.12).

\subsubsection{Generalized 2-chorded cycles}

In this subsection we show that 2-chorded cycles plus some additional 3-chords (with coefficient 1) and some 4-chords (with coefficient -1) also define facets of the clique partitioning polytope associated with complete graphs.

Lemma 3.11 Let $K_{n}=\left(V_{n}, E_{n}\right)$ be a complete graph, let $C \subseteq E_{n}$ be an odd cycle of length $k \geq 6$ in $K_{n}, V_{n}(C)=\left\{v_{1}, \ldots, v_{k}\right\} \subseteq V_{n}$, and let $C=$ $\mathrm{Ch}_{2}(C)$ be the set of 2-chords of $C$. Furthermore, let $D \subseteq \mathrm{Ch}_{3}(C)$ and let $\bar{D}=\mathrm{Ch}_{2}(C \cup D) \backslash \bar{C}$, such that $D \cap \bar{D}=\varnothing$. The generalized 2 -chorded cycle inequality

$$
x(C \cup D)-x(\bar{C} \cup \bar{D}) \leq\left\lfloor\frac{1}{2}|C|\right\rfloor
$$

is valid for $P\left(K_{n}\right)$. Moreover, for any facet $F_{b}=\left\{x \in P\left(K_{n}\right) \mid b^{T^{x}} x=b_{0}\right\}$ 
containing the face induced by (3.17) the following holds.

$$
b_{i j}=\left\{\begin{aligned}
\beta & \forall\{i, j\} \in C \cup D \\
-\beta & \forall\{i, j\} \in \bar{C} \cup \bar{D} \\
0 & \forall\{i, j\} \in \mathrm{Ch}_{p}(C) \quad(p \geq 5) \\
0 & \forall\{i, j\} \notin E_{n}\left(V_{n}(C)\right)
\end{aligned}\right.
$$

for some $\beta>0$.

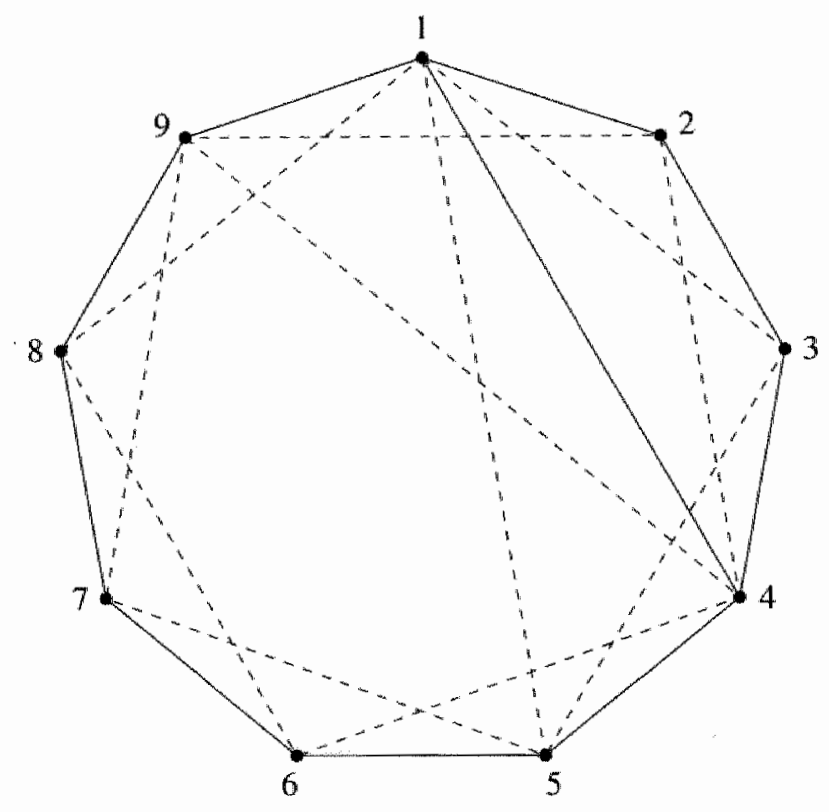

Figure 3.3: Support of a generalized 2-chorded cycle inequality.

Proof Validity of (3.17) immediately follows from Lemma 2.17. To prove that (3.18) holds we proceed as folllows. Denote (3.17) by $a^{T} x \leq a_{0}$, and for all $v_{i} \in V_{n}(C)$ define the following matchings contained in $C \cup D$.

$$
M_{i}=\left\{\left\{v_{i}, v_{i+1}\right\},\left\{v_{i+2}, v_{i+3}\right\}, \ldots,\left\{v_{i+k-3}, v_{i+k-2}\right\}\right\}
$$

Moreover, if $\left\{v_{i}, v_{i+3}\right\} \in D$ construct $M_{i}^{\prime}$ from $M_{i}$ by replacing the edges $\left\{v_{i}, v_{i+1}\right\}$ and $\left\{v_{i+2}, v_{i+3}\right\}$ by $\left\{v_{i}, v_{i+3}\right\}$ and $\left\{v_{i+1}, v_{i+2}\right\}$. The incidence vectors 
of all these matchings belong to the face induced by (3.17). Since $M_{i} \triangle M_{i+2}=$ $\left\{\left\{v_{i}, v_{i-1}\right\},\left\{v_{i}, v_{i+1}\right\}\right\}$ it follows that $b_{v_{i}, v_{i-1}}=b_{v_{i}, v_{i+1}}$, or equivalently $b_{i j}=\beta$ for all $\{i, j\} \in C$ and some $\beta>0$. Comparing $M_{i}$ and $M_{i}^{i}$ yields $b_{i j}=\beta$ for all $\{\dot{i}, j\} \in D$.

Next, define $\tilde{M}_{i}=M_{i} \cup\left\{\left\{v_{i}, v_{i-1}\right\},\left\{v_{i-1}, v_{i+1}\right\}\right\}$. From $a^{T} \chi^{\tilde{M}_{i}}=a_{0}$ it follows that $b_{v_{i-1}, v_{i+1}}=-b_{v_{i}, v_{i+1}}=-\beta$ for all $v_{i} \in V_{n}(C)$. Define $\tilde{M}_{i}^{\prime}=$ $M_{i}^{\prime} \cup\left\{\left\{v_{i}, v_{i-1}\right\},\left\{v_{i-1}, v_{i+3}\right\}\right\}$. Since $a^{T} \chi^{M_{i}^{\prime}}=a_{0}$ it follows that $b_{v_{i-1}, w_{i+3}}=$ $-b_{v_{i}, v_{i+1}}=-\beta$. Symmetry implies that $b_{i j}=-\beta$ for all $\{i, j\} \in D$.

In a very similar fashion as in [39] it can be shown that $b_{i j}=0$ for all $\{i, j\} \in$ $\mathrm{Ch}_{p}(C)$ for $p \geq 5$. If $i, j \in V_{n}$ are not incident to $C$, then obviously $b_{i j}=0$. If $v_{i} \in V_{n}(C)$ and $w \in V_{n} \backslash V_{n}(C)$, then both $M_{i+1}$ and $M_{i+1} \cup\left\{\left\{v_{i}, w\right\}\right\}$ satisfy (3.17) at equality, and hence $b_{v_{i}, w}=0$. This completes the proof of the lemma.

In some situations inequality (3.17) defines a facet of $P\left(K_{n}\right)$, for instance if $D$ consists of 1,2 , or 3 consecutive 3 -chords of $C$. This is shown in the next theorem.

Theorem 3.12 Let $K_{n}=\left(V_{n}, E_{n}\right)$ be a complete graph, let $C \subseteq E_{n}$ be a cycle of length $k \geq 7$ in $K_{n}, V_{n}(C)=\left\{v_{1}, \ldots, v_{k}\right\} \subseteq V_{n}$, and let $\bar{C}=\mathrm{Ch}_{2}(C)$ be the set of 2-chords of $C$. Furthermore, for $v_{i} \in V_{n}(C)$ let $D \subseteq \mathrm{Ch}_{3}(C)$ be any of the following sets (indices modulo $k$ ),

(i) $D=\left\{\left\{v_{i}, v_{i+3}\right\}\right\}$ (if $\left.k \geq 9\right)$

(ii) $D=\left\{\left\{v_{i}, v_{i+3}\right\},\left\{v_{i+1}, v_{i+4}\right\}\right\}$ (if $k \geq 9$ )

(iii) $D=\left\{\left\{v_{i}, v_{i+3}\right\},\left\{v_{i+1}, v_{i+4}\right\},\left\{v_{i+2}, v_{i+5}\right\}\right\}$ (if $k \geq 7$ )

and let $\bar{D}=\mathrm{Ch}_{2}(C \cup D) \backslash \bar{C}$. The generalized 2-chorded cycle inequality

$$
x(C \cup D)-x(\bar{C} \cup \bar{D}) \leq\left\lfloor\frac{1}{2}|C|\right\rfloor
$$

is valid for $P\left(K_{n}\right)$. It defines a facet if and only if $|C|$ is odd.

Proof Validity of (3.19) immediately follows from Lemma 2.17, and a little extension shows that (3.19) does not define a facet of $P\left(K_{n}\right)$ if $|C|$ is even. 
Denote (3.19) by $a^{T} x \leq p$, let $F_{a}$ be the face of $P\left(K_{n}\right)$ induced by this inequality, and let $M \subseteq E_{n}$ be a clique partition containing $p+q$ edges in $C \cup D$ for some integer $q \geq 1$. Since $|C|=k$ there are at least

$$
2(p+q)-k= \begin{cases}2 q, & k \text { even } \\ 2 q-1, & k \text { odd }\end{cases}
$$

pairs of edges in $M \cap(C \cup D)$ that share a vertex. Since all 2-chords of $C \cup D$ belong to $\bar{C} \cup \bar{D}$ we have

$$
a^{T} \chi^{M} \leq \begin{cases}p+q-2 q=p-q<p, & k \text { even } \\ p+q-(2 q-1)=p-q+1 \leq p, & k \text { odd }\end{cases}
$$

If $k$ is even and $a^{T} \chi^{M}=p$, then $M$ contains no edges in $\bar{C} \cup \bar{D}$, and the face induced by (3.19) is contained in each of the hyperplanes $x_{i j}=0 \quad\{i, j\} \in$ $\tilde{C} \cup \bar{D})$, which implies that $(3.19)$ does not define a facet of $P\left(K_{n}\right)$.

In the remainder of this proof we assume that $C$ is an odd cycle. Let $F_{b}:=$ $\left\{x \in P\left(K_{n}\right) \mid b^{T} x=b_{0}\right\}$ be a facet containing $F_{a}$. Lemma 3.11 shows that there exists $\beta>0$ such that $b_{i j}=\beta$ for all $\{i, j\} \in C \cup D, b_{i j}=-\beta$ for all $\{i, j\} \in \bar{C} \cup \bar{D}$, and $b_{i j}=0$ for all $r$-chords $\{i, j\}$ of $C(r \geq 5)$ and all edges $\{i, j\} \in E_{n}$ incident to a vertex outside $V_{n}(C)$.

It remains to show that $b_{i j}=0$ for all 3 -chords and 4-chords $\{i, j\} \in E_{n}(C)$ not contained in $\bar{C} \cup \bar{D}$. In case (iii), if $k=7$, then $E_{\text {fn }}(C)=C \cup \bar{C} \cup D \cup \bar{D}$, and hence (3.11) defines a facet. In the remainder of the proof we assume that $k \geq 9$, and without loss of generality we also assume that $v_{i}=v_{1}$. Taking indices modulo $k$, consider

$$
\begin{aligned}
& A_{j}^{\prime}:=\left\{\left\{v_{j}, v_{j+3}\right\},\left\{v_{j}, v_{j+4}\right\}\right\} \cup M_{j} \\
& A_{j}^{\prime \prime}:=\left\{\left\{v_{j}, v_{j+4}\right\},\left\{v_{j+1}, v_{j+4}\right\}\right\} \cup M_{j+4}
\end{aligned}
$$

$(j=|D|+1, \ldots, k-1)$ where $M_{j}$ is the $\left\lfloor\frac{1}{2}|C|\right\rfloor$-matching in $C$ not covering $v_{j}$. Since $\chi^{A_{j}}, \chi^{A_{j}^{\prime \prime}} \in F_{a} \subseteq F_{b}$ and $b_{i j}=\beta$ for all $\{i, j\} \in C$, it follows that

$$
b_{v_{j}, n_{j+3}}=\beta^{\prime} \quad \forall j=|D|+1, \ldots, k
$$


for some $\beta^{\prime} \in \mathbb{R}$. Further, by comparing $\chi^{A_{j}^{\prime}}$ and $\chi^{M_{j}}$ it follows that

$$
b_{v_{j}, v_{j+4}}=-\beta^{\prime} \quad \forall j=|D|+1, \ldots, k-1
$$

Next, consider the clique partitions

$$
\begin{aligned}
& B^{\prime}:=E\left(\left\{v_{1}, v_{4}, v_{7}\right\}\right) \cup\left\{\left\{v_{2}, v_{3}\right\},\left\{v_{5}, v_{6}\right\}\right\} \cup\left(\bigcup_{j=4}^{p}\left\{\left\{v_{2 j}, v_{2 j+1}\right\}\right\}\right) \\
& B^{\prime \prime}:=\left\{\left\{v_{1}, v_{4}\right\},\left\{v_{2}, v_{3}\right\},\left\{v_{5}, v_{6}\right\}\right\} \cup\left(\bigcup_{j=4}^{p}\left\{\left\{v_{2 j}, v_{2 j+1}\right\}\right\}\right)
\end{aligned}
$$

Clearly, $\chi^{B^{\prime}}, \chi^{B^{\prime \prime}} \in F_{a} \subseteq F_{b}$, so we can conclude that $b_{v_{1}, v_{7}}+b_{v_{4}, v_{7}}=0$. If $k=9$, then the edges $\left\{v_{1}, v_{7}\right\}$ and $\left\{v_{4}, v_{7}\right\}$ are both 3 -chords of $C$ not contained in $D$, so from (3.20) we can conclude that $b_{v_{1}, v_{7}}+b_{v_{4}, v_{7}}=2 \beta^{\prime}$, and hence $\beta^{\prime}=0$. If $k \geq 11$, then we already know that $\beta_{v_{1}, v_{7}}=0$, so we can immediately conclude that $b_{v_{4}, v_{7}}=\beta^{\prime}=0$. This completes the proof of the theorem.

With a little extra effort we can deduce the following corollary.

Corollary 3.13 Let $K_{n}=\left(V_{n}, E_{n}\right)$ be a complete graph, let, $C \subseteq E_{n}$ be an odd cycle of length $k \geq 9$ in $K_{n}, V_{n}(C)=\left\{v_{1}, \ldots, v_{k}\right\} \subseteq V_{n}$, and let $\vec{C}=$ $\mathrm{Ch}_{2}(C)$ be the set of 2-chords of $C$. Furthermore, let $D_{1}, \ldots, D_{k} \subseteq \mathrm{Ch}_{3}(C)$ be like edge set $D$ in Theorem 3.12, and let $\bar{D}_{i}=\mathrm{Ch}_{2}\left(C \cup D_{i}\right) \backslash \bar{C}(i=1, \ldots, k)$, such that $V_{n}\left(D_{i} \cup \bar{D}_{i}\right) \cap V_{n}\left(D_{j} \cup \bar{D}_{j}\right)=\varnothing$ for all $1 \leq i<j \leq k$. Finally, let $D:=D_{1} \cup \ldots \cup D_{k}$ and $\bar{D}:=\bar{D}_{1} \cup \ldots \cup \bar{D}_{k}$. Then inequality (3.19) defines a facet of $P\left(K_{n}\right)$.

Proof Along the same lines as the proof of Theorem 3.12. 


\subsubsection{Generalized path and wheel inequalities}

The 2-chorded path and the 2-chorded wheel inequalities (see Theorem 3.3) can also be generalized, as is witnessed by the two theorems in this subsection.

Theorem 3.14 Let $P=\left\{\left\{u_{i}, u_{i+1}\right\} \mid i=1, \ldots, k\right\}$ be an even path of length $k \geq 2$, let $\bar{P}=\mathrm{Ch}_{2}(P)$, and let $Z \subseteq V_{n}$ be a set of vertices disjoint from $V_{n}(P)$. Define

$$
\begin{aligned}
& R:=\left\{\left\{z, u_{i}\right\} \mid z \in Z, i=2, \ldots, k, i \text { even }\right\} \\
& \ddot{R}:=\left\{\left\{z, u_{i}\right\} \mid z \in Z, i=1, \ldots, k+1, i \text { odd }\right\}
\end{aligned}
$$

The inequality

$$
x(P \cup R)-x(\bar{P} \cup \bar{R})-x\left(E_{\pi}(Z)\right) \leq\left\lfloor\frac{1}{2}(|P|+1)\right\rfloor
$$

is vallid and facet defining for $P\left(K_{n}\right)$.

Proof Validity: all edges in $P \cup R$ are incident to a node in $U:=\left\{u_{2 i} \mid i=\right.$ $\left.1, \ldots, \frac{1}{2} k\right\}$, and hence there are no more than $\frac{1}{2} k$ vertex-disjoint edges in $P \cup R$. Moreover, if two edges in $P \cup R$ have a common vertex in $U$, the 2-chord corresponding to these edges belongs to $\bar{P} \cup \bar{R} \cup E_{n}(Z)$. If $A \subseteq E_{n}$ is a clique partition containing $\frac{1}{2} k+q$ edges in $P \cup R$ for some $q \geq 1$, then $A$ also contains at least $q$ edges in $\bar{P} \cup \bar{R} \cup E_{n}(Z)$, hence (3.22) is valid.

Facetness: denote (3.22) by $a^{T} x \leq a_{0}$. If $|Z|=1$, then (3.22) is simply a 2chorded path inequality, which is known to be facet defining. If $|Z| \geq 2$ define $E_{z}:=E_{n}\left(V_{n}(P) \cup\{z\}\right)$ for all $z \in Z$, and apply Theorem 2.13. The load of $E_{z}$ with respect to $a^{T} x \leq a_{0}$ is equal to $a_{0}$ since the 2 -chorded path inequality defines a facet of $P\left(K_{n}\right)$, and hence the residual equals 0 . Theorem 2.13 proves that (3.22) defines a facet of $P\left(V_{n}, E_{n} \backslash E_{n}(Z)\right)$. To prove that it also defines a facet of $P\left(K_{n}\right)$ it suffices to identify for each $\left\{z_{1}, z_{2}\right\} \in E_{n}(Z)$ a clique partition containing this edge, but not containing any other edge in $E_{n}(Z)$. Let $z_{1}, z_{2} \in Z$ be two different vertices, and let

$$
M:=E_{n}\left(\left\{u_{2}, z_{1}, z_{2}\right\}\right) \cup\left\{\left\{u_{3}, u_{4}\right\}, \ldots,\left\{u_{k-2}, u_{k-1}\right\}\right\}
$$

Then $a^{T} \chi^{M}=a_{0}$, and $M \cap E_{n}(Z)=\left\{z_{1}, z_{2}\right\}$. 
Theorem 3.15 Let $C \subseteq E_{n}$ be an even cycle of length $k \geq 8$ in $K_{n}$ with $V_{n}(C)=\left\{u_{1}, \ldots, u_{k}\right\}$, let $\bar{C}=\mathrm{Ch}_{2}(C)$, and let $Z \subseteq V_{n}$ be a set of vertices disjoint from $V_{n}(C)$. Define $R$ and $\bar{R}$ as in Theorem 3.14, then the generalized 2-chorded wheel inequality

$$
x(C \cup R)-x(\bar{C} \cup \bar{R})-x\left(E_{n}(Z)\right) \leq \frac{1}{2}|C|
$$

is valid and facet defining for $P\left(K_{n}\right)$.

Proof Along the same lines as the proof of Theorem 3.14. $\square$

Inequalities (3.22) can be generalized even further as follows.

Theorem 3.16 Let $Q=\left\{\left\{u_{i}, u_{i+1}\right\} \mid i=2, \ldots, k-1\right\}$ be an even path of length $k-2$, and let $U_{1}, U_{k+1}, Z \subseteq V_{n} \backslash V_{n}(Q)$ be three nonempty disjoint sets. Further, define $P=E_{n}\left(U_{1},\left\{u_{2}\right\}\right) \cup Q \cup E_{n}\left(\left\{u_{k}\right\}, U_{k+1}\right), \bar{P}=\mathrm{Ch}_{2}(P)$, and

$$
\begin{aligned}
& R:=\left\{\left\{z, u_{i}\right\} \mid z \in Z, i=2, \ldots, k, i \text { even }\right\} \\
& \bar{R}:=\left\{\left\{z, u_{i}\right\} \mid z \in Z, i=3, \ldots, k-1, i \text { odd }\right\} \cup E_{n_{3}}\left(U_{1} \cup U_{k+1}, Z\right)
\end{aligned}
$$

The generalized 2-chorded path inequality

$$
x(P \cup R)-x(\bar{P} \cup \bar{R})-x\left(E_{n}(Z)\right) \leq\left\lfloor\frac{1}{2}(|Q|+3)\right\rfloor
$$

is valid and facet defining for $P\left(K_{n}\right)$.

Proof Straightforward. 


\subsection{Lifting}

In the previous section we described several classes of facet defining inequalities for the clique partitioning polytope. In this section we describe a lifting technique that can be used to transform facet defining inequalities into new facet defining inequalities.

Let $H=(U, F)$ be a subgraph of the graph $G=(V, E)$, i.e. $U \subseteq V$ and $F \subseteq E(U)$. If $a^{T} x \leq a_{0}$ defines a facet of the clique partitioning polytope corresponding to $H$, then by sequentially lifting the coefficients of the edges in $E \backslash F$, we find a facet defining inequality of the clique partitioning polytope corresponding to $G$ (see Wolsey $[65,66]$ for sequentially lifting the facets of general polytopes). This is stated more precisely in the following lemma.

\section{Lemma 3.17 (Sequential lifting [65])}

Let $G=(V, E)$ be a graph, $e \in E$, and let $a^{T} x \leq a_{0}$ be a facet defining inequality for the polytope $\left\{x \in P(G) \mid x_{e}=0\right\}$. Define $\tilde{a} \in \mathbb{R}^{|E|}$ by

$$
\tilde{a}_{i j}=\left\{\begin{array}{l}
a_{i j} \quad \text { if }\{i, j\} \in E \backslash\{e\} \\
a_{0}-\max \left\{a^{T} x \mid x \in P(G), x_{e}=1\right\} \quad \text { if }\{i, j\}=e
\end{array}\right.
$$

Then the inequality $\tilde{a}^{T} x \leq a_{0}$ defines a facet of $P(G)$.

If $G=(V, E)$ and $F \subseteq E$, then by repeatedly applying Lemma 3.17 one can derive facets of $P(G)$ from known facets of $P(V, E \backslash F)$. In general, the optimization problem in (3.25) is difficult to solve. However, in some special cases (see Lemma 3.18) it can be quite easy. Sometimes, lifting a valid inequality which is not facet defining for $\left\{x \in P(G) \mid x_{e}=0\right\}$ results in a facet defining inequality for $P(G)$.

For instance, if $F=E(\{v\}, V \backslash\{v\})$, then lifting the upper bound constraint $x_{i, j} \leq 1(i, j \in V \backslash\{v\})$ (which does not define a facet, unless $\{i, j\}$ is an isolated edge) results in a triangle inequality, no matter in what order the coefficients are lifted. However, in general lifting the coefficients of the edges in $F$ in different orders results in different facets.

Let us refer to an inequality where one node (called the center) is incident to all edges with positive coefficients, as a star inequality. Claw inequalities and $(S, T)$-inequalities with $|S|=1$ are examples of star inequalities. 
Lemma 3.18 Let $K_{n}=\left(V_{n}, E_{\eta_{n}}\right)$ be a complete graph, $V_{n}=\{s\} \cup T_{1} \cup T_{2}$, and let $a^{T} x \leq a_{0}$ be a facet defining star inequality for the clique partitioning polytope corresponding to $K^{\prime}=\left(\{s\} \cup T_{1}, E_{n}\left(\{s\} \cup T_{1}\right)\right)$ with the property that $a_{i j}>0$ if and only if $\{i, j\} \in E_{n}\left(\{s\}, T_{1}\right)$. Define $\bar{a} \in \mathbb{R}^{\left|E_{n}\right|}$ by

$$
\bar{a}_{i j}=\left\{\begin{aligned}
a_{i j} & \forall\{i, j\} \in E_{n}\left(\{s\} \cup T_{1}\right) \\
a_{0} & \forall\{i, j\} \in E_{n}\left(\{s\}, T_{2}\right) \\
-a_{0} & \forall\{i, j\} \in E_{n}\left(T_{2}\right) \\
-a_{s i} & \forall i \in T_{1}, j \in T_{2}
\end{aligned}\right.
$$

Then the inequality $\bar{a}^{T} x \leq a_{0}$ is valid and facet defining for $P\left(K_{n}\right)$.

Proof First, we show that $\bar{a}^{T} x \leq a_{0}$ is valid for $P\left(K_{n}\right)$. Let $A \subseteq E_{n}$ be a clique partition, and let $A^{\prime} \subseteq A$ be the clique in $A$ incident to $s$. Since all edges $\{i, j\} \in E_{n}$ with $a_{i j}>0$ are incident to $s$, we have that $\bar{a}^{T} \chi^{A} \leq \bar{a}^{T} \chi^{A^{\prime}}$. If $V_{n}\left(A^{\prime}\right) \cap T_{1}=\varnothing$, then $\bar{a}^{T} \chi^{A^{\prime}} \leq a_{0}$, because $A^{\prime}$ satisfies the $(S, T)$-inequality induced by $S=\{s\}$ and $T=T_{2}$. If $V_{n}\left(A^{\prime}\right) \cap T_{1} \neq \varnothing$, then we distinguish three situations:

(i) $\left|V_{n}\left(A^{\prime}\right) \cap T_{2}\right|=0$ : Then $\bar{a}^{T} \chi^{A^{\prime}}=a^{T} \chi^{A^{r}} \leq a_{0}$.

(ii) $\left|V_{n}\left(A^{\prime}\right) \cap T_{2}\right|=1$ : Then

$$
\begin{aligned}
\bar{a}^{T} \chi^{A^{r}} & =a_{0}+\sum_{\substack{i \in T_{1} \cap V_{n n}\left(A^{\prime}\right)\\
}} a_{s i}-\sum_{i \in T_{1} \cap V_{n}\left(A^{\prime}\right)} a_{s i}+\sum_{\substack{i, j \in T_{1} \cap V_{n}\left(A^{\prime}\right) \\
i<j}} a_{i j} \\
& =a_{0}+\sum_{\substack{i, j \in T_{1} \cap V_{n}\left(A^{\prime}\right) \\
i<j}} a_{i j} \leq a_{0}
\end{aligned}
$$

(iii) $\left|V_{n}\left(A^{\prime}\right) \cap T_{2}\right| \geq 2$ : Let $t \in V_{n}\left(A^{\prime}\right) \cap T_{2}$, and let $B:=E_{n}\left(\{s, t\} \cup\left(V_{n}\left(A^{\prime}\right) \cap\right.\right.$ $\left.T_{1}\right)$ ). Then $\bar{a}^{T} \chi^{A^{\prime}} \leq \bar{a}^{T} \chi^{B} \leq a_{0}$, since $B$ is a clique with $\left|V_{n}(B) \cap T_{2}\right|=1$. This shows that $\bar{a}^{T} x \leq a_{0}$ is valid.

To prove that it is facet defining, we repeatedly apply Lemma 3.17 to the edges in $E_{n} \backslash E_{n}\left(\{s\} \cup T_{1}\right)$ in the following order.

(i) Edges in $E_{n}\left(\{s\}, T_{2}\right)$,

(ii) Edges in $E_{n}\left(T_{2}\right)$, 
(iii) $\mathbb{E} d g e s$ in $E_{n}\left(T_{1}, T_{2}\right)$.

This yields the following result.

(i) The validity of $\bar{a}^{T} x \leq a_{0}$ shows that we can lift the coefficients of all edges in $E_{n}\left(\{s\}, T_{2}\right)$ to $a_{0}$, and it is obvious that no coefficient can be lifted to more than $a_{0}$.

(ii) Let $i, j \in T_{2}$. Since $E_{n}(\{s, i, j\})$ is a clique partition, it follows that the coefficient of $\vec{a}_{i j}$ cannot be lifted to more than $a_{0}-2 a_{0}=-a_{0}$. The validity of $\bar{a}^{T} x \leq a_{0}$ shows that $\bar{a}_{i j}$ can indeed by lifted to $-a_{0}$.

(iii) Let $i \in T_{1}$ and $j \in T_{2}$. Because of the triangle $E_{n}(\{s, i, j\})$ the coefficient of $\{i, j\}$ cannot be lifted to more than $-a_{s i}$, and the validity of $\bar{a}^{T} x \leq a_{0}$ shows that: it can indeed by lifted to $-a_{s i}$.

Inequalities satisfying the condition of Lemma 3.18 are the claw inequalities and the stable set inequalities, hence we have the following two corollaries.

Corollary 3.19 Let $K_{n}=\left(V_{n}, E_{n}\right)$ be a complete graph $\{s\}, T_{1}, T_{2} \subseteq V_{n}$ be disjoint sets, and let $c \geq 2$. The lifted claw inequality

$$
\begin{aligned}
& c \cdot x\left(E_{n}\left(\{s\}, T_{1}\right)\right)+\left(\begin{array}{c}
c+1 \\
2
\end{array}\right) \cdot x\left(E_{n}\left(\{s\}, T_{2}\right)\right)-x\left(E_{n}\left(T_{1}\right)\right) \\
& -\left(\begin{array}{c}
c+1 \\
2
\end{array}\right) \cdot x\left(E_{n}\left(T_{2}\right)\right)-c \cdot x\left(E_{n}\left(T_{1}, T_{2}\right)\right) \leq\left(\begin{array}{c}
c+1 \\
2
\end{array}\right)
\end{aligned}
$$

defines a facet of $P\left(K_{n}\right)$.

Proof This follows from Theorem 3.7 and Lemma 3.18.

Notice that for each $n \in \mathbb{N}$ there is a lifted claw inequality with coefficients equal to $n$ and coefficients equal to $-n$. Furthermore, the polytope $P\left(K_{n}\right)$ has facets with coefficients in $O\left(n^{2}\right)$.

Corollary 3.20 Let $K_{n}=\left(V_{n}, E_{n}\right)$ be a complete graph, $\{s\}, T_{1}, T_{2} \subseteq V_{n}$ be disjoint sets, and let $H=\left(T_{1}, F\right)$ be a graph satisfying conditions (i)-(vi) of Theorem 3.9. The lifted stable set inequality

$$
\begin{aligned}
& x\left(E_{n}\left(\{s\}, T_{1}\right)\right)-x(F)+\alpha(H) \cdot x\left(E_{n}\left(\{s\}, T_{2}\right)\right) \\
& -\alpha(H) \cdot x\left(E_{n}\left(T_{2}\right)\right)-x\left(E_{n}\left(T_{1}, T_{2}\right)\right) \leq \alpha(H)
\end{aligned}
$$


defines a facet of $P\left(K_{n}\right)$.

Proof This follows from Theorem 3.9 and Lemma 3.18. $\square$

\subsection{Patching}

It is often possible to use a set of valid inequalities to construct a new valid inequality that is not implied by the former. Well-known examples are the procedures to derive Gomory-Chvátal cuts [16], node cloning for the traveling salesman problem (Balas and Fischetti [4]), and contraction or subdivision of edges (see Barahona et al. [5] for the bipartite subgraph polytope, see Barahona and Mahjoub [6] for the cut polytope). Another technique to derive new facets is graph substitution, see for instance Balas and Zemel [3] for set packing polytopes. In this section we focus on so-called patching procedures. A patching procedure combines a set of inequalities into a single one such that the nonzero coefficients of these inequalities remain unchanged.

Here we restrict ourselves to combining pairs of inequalities and we distinguish two cases of patching, depending on the intersection of the node sets of the supports of the inequalities involved. If the intersection is empty, we refer to combining the two inequalities as disjoint patching, otherwise we call it intersection patching. In the case of intersection patching the two inequalities must have identical coefficients for all edges in the intersection.

Grötschel and Wakabayashi [40] present some patching procedures for the class of 2-partition inequalities. One of their procedures can informally be described as follows. Take nonempty sets $S_{1}, S_{2}, T_{1}, T_{2} \subseteq V_{n}$, pairwise disjoint, except for $T:=T_{1} \cap T_{2}$. Assume that $|T| \geq 2,\left|S_{1}\right| \leq\left.\left|T_{1}\right| T\right|_{\text {, and }}\left|S_{2}\right| \leq\left|T_{2} \backslash T\right|$. Then the facet defining $(S, T)$-inequalities

$$
x\left(E_{n}\left(S_{1}, T_{1}\right)\right)-x\left(E_{n}\left(S_{1}\right)\right)-x\left(E_{n}\left(T_{1}\right)\right) \leq\left|S_{1}\right|
$$

and

$$
x\left(E_{n}\left(S_{2}, T_{2}\right)\right)-x\left(E_{n}\left(S_{2}\right)\right)-x\left(E_{n}\left(T_{2}\right)\right) \leq\left|S_{2}\right|
$$


can be combined to

$$
\begin{aligned}
& x\left(E_{n}\left(S_{1}, T_{1}\right)\right)+x\left(E_{n}\left(S_{2}, T_{2}\right)\right)-x\left(E_{n}\left(S_{1} \cup S_{2}\right)\right) \\
& -x\left(E_{n}\left(T_{1}\right)\right)-x\left(E_{n}\left(T_{2}\right)\right)+x\left(E_{n}(T)\right) \leq\left|S_{1}\right|+\left|S_{2}\right|
\end{aligned}
$$

which is also facet defining. Notice that compared to just adding up the two $(S, T)$-inequalities, the coefficients of the variables in $E_{n}(T)$ and $E_{n}\left(S_{1}, S_{2}\right)$ are raised, respectively lowered, by one.

In the remainder of this subsection we present several new patching procedures for the clique partitioning polytope. These procedures need the concept of a covering, which in turn requires the concept of the residual, as given in Definition 2.10 in the previous chapter.

Definition 3.21 The covering of a node set $U \subseteq V_{n}$ with respect to the inequality $a^{T} x \leq a_{0}$, denoted by $\operatorname{cov}(a, U)$, is defined as

$$
\operatorname{cov}(a, U):=a_{0}-\operatorname{res}_{K_{n}}\left(a, E_{n}\left(V_{n} \backslash U\right)\right)
$$

Intuitively, the covering of a vertex set $U \subseteq V_{n}$ with respect to an inequality is the maximal contribution of the edges incident to $U$ to the left-hand side. The covering of $U$ is not the same as the load of $E(U)$. Consider for instance a star inequality $a^{T} x \leq a_{0}$ with center $s \in V_{n}$. Then $\operatorname{load}_{K_{n}}(a, E(\{s\}))=0$ because $E(\{s\})$ is empty, but $\operatorname{cov}(a,\{s\})=a_{0}$ since all edges $\{i, j\} \in E_{n}$ with $a_{i j}>0$ are incident to $\mathrm{s}$. Although some of the forthcoming results in this section can also be derived by using the load, we feel that the covering is better suited for describing the disjoint patching procedures.

As an illustration of covering, consider the triangle inequality $x_{12}+x_{13}-x_{23} \leq$ 1. It is easy to verify that the covering of any node set containing node 1 equals 1. Notice that the covering of any node set $U \subseteq V_{n}$ is nonnegative, assuming that the inequality is valid. Further, the covering of a single node $s$ equals zero if and only if there exists a clique partition not incident to $s$ satisfying $a^{T} x \leq a_{0}$ at equality. Finally, the definition implies that the following inequalities are valid:

$$
\sum_{\{i, j\} \in E_{n}\langle V \backslash U)} a_{i j} x_{i j} \leq a_{0}-\operatorname{cov}(a, U)
$$




$$
\sum_{\{i, j\} \in E_{n}(U)} a_{i j} x_{i j} \leq \operatorname{cov}(a, U)
$$

Based on this concept we can make an interesting observation, that seems to suggest that the patching approach may be well suited for investigating the facial structure of the clique partitioning polytope. Intuitively, we show that it is possible to use 'small' facet defining inequalities as the core of larger lacet defining inequalities.

Lemma 3.22 (Basic patching principle)

Let $K_{n}=\left(V_{n}, E_{n}\right)$ be the complete graph on $n$ vertices, let $V_{C}$ be an arbitrary nonempty subset of $V_{n}$, and let $E^{\prime}$ be a (possibly empty) arbitrary subset of $E_{n}\left(V_{C}, V_{n} \backslash V_{C}\right)$. Let $P^{*}$ be the face of $P\left(K_{n}\right)$ defined by the equalities $x_{i j}=0$ for all $\{i, j\} \in E^{\prime}$. Given is a valid inequality $\pi^{T} x \leq \pi_{0}$, defining a proper face $F_{\pi}$ of $P^{*}$, such that

$$
\rho^{T^{\prime}} x:=\sum_{\{i, j\} \in E_{n}\left(V_{C}\right)} \pi_{i j} x_{i j} \leq \operatorname{cov}\left(\pi, V_{C}\right)
$$

defines a nontrivial facet of $P\left(K_{n}\right)$.

Then there exists a facet $F=\left\{x \in P^{*} \mid \delta^{T} x=\delta_{0}\right\}$ of $P^{*}$ containing $F_{\pi}$, such that

(i) $\delta_{i j} \neq 0$ for at least one edge $\{i, j\} \in E_{n}\left(V_{C}\right)$,

(ii) there is a $\beta>0$ such that $\delta_{i j}=\beta \cdot \pi_{i j}$ for all $\{i, j\} \in E_{n}\left(V_{C}\right)$.

Proof The inequality $\rho^{T} x \leq \operatorname{cov}\left(\pi, V_{C}\right)$ defines a facet, so there must exist: $\pi_{i j} \neq 0$ for some $\{i, j\} \in E_{n}\left(V_{C}\right)$. It follows, since $\pi^{T} x \leq \pi_{0}$ defines a proper face of $P^{*}$, that there exists a facet of $P^{*}$, containing $F_{\pi}$, defined by some inequality $\delta^{T} x \leq \delta_{0}$ for which $\hat{\delta}_{i j} \neq 0$.

To proceed, take a maximal affinely independent set of feasible integer solutions satisfying $\rho^{T} x \leq \rho_{0}:=\operatorname{cov}\left(\pi, V_{C}\right)$ at equality. Let $M$ be $\operatorname{dim}\left(P\left(K_{n}\right)\right) \times$ $\operatorname{dim}\left(P\left(K_{n}\right)\right)$ matrix such that there is precisely one column for every solution of the set, and each column represents the incidence vector of the corresponding solution. Then $\rho^{T} M=p_{0} \cdot 1$. 
Let $\rho_{C}, x_{C}$ be vectors with the elements of $\rho$, respectively $x$, that correspond to the edges in $E_{n}\left(V_{C}\right)$. There exist $\left|E_{n}\left(V_{C}\right)\right|$ columns such that the rows in these columns corresponding to the elements of $E_{n}\left(V_{C}\right)$ form a matrix $M_{C}$ that is nonsingular. Since the coefficients of $\rho$ corresponding to edges covering nodes not in $V_{C}$ are all zero, we have: $\rho_{C}^{T} M_{C}=\rho_{0} \cdot \mathbf{1}$, implying

$$
\rho_{C}=\rho_{0} \cdot M_{C}^{-1} \cdot \mathbf{1}
$$

Notice that $\rho_{0}>0$ since $\rho^{T^{*}} x \leq \rho_{0}$ is assumed to define a nontrivial facet.

Now, let $y$ be a feasible solution such that $\pi^{T} y=\pi_{0}-\operatorname{cov}\left(\pi, V_{C}\right)$, and $y_{i j}=0$ for all $\{i, j\} \in E_{n}\left(V, V_{C}\right)$. Such a solution always exists due to the definition of a covering. Then, for each solution $x$ corresponding to a column of $M_{C}$, we construct a solution $z$ as follows.

$$
z_{i j}= \begin{cases}x_{i j} & \text { if }\{i, j\} \in E_{n}\left(V_{C}\right) \\ y_{i j} & \text { if }\{i, j\} \in E_{n}\left(V \backslash V_{C}\right) \\ 0 & \text { otherwise }\end{cases}
$$

Notice that $z$ is in $P^{*}$. Further, it is easy to verify that for each solution $z$ we have $\pi^{T} z=\rho_{0}+\pi_{0}-\operatorname{cov}\left(\pi, V_{C}\right)=\pi_{0}$. In other words, when $\tilde{M}$ refers to a $\left|E_{n_{i}}(V)\right| \times\left|E_{n}\left(V_{C}\right)\right|$ matrix where each column represents the incidence vector of a solution $z$, we have that $\pi^{T} \tilde{M}=\pi_{0} \cdot \mathbf{1}$, and since $F$ contains $F_{\pi}$ also $\delta^{T} \tilde{M}=\delta_{0} \cdot \mathbf{1}$.

The construction of the solutions $z$ implies that the rows in $\tilde{M}$ corresponding to the elements of $E_{n}\left(V_{C}\right)$ form the nonsingular matrix $M_{C}$. Notice further that for all columns in $\tilde{M}$, the part of each column not in $M_{C}$ is identical. Let $\delta_{C}$ be the vector with the elements of $\delta$ that correspond to the edges in $E_{n}\left(V_{C}\right)$. We derive: $\delta^{T} \tilde{M}=\delta_{C}^{T} M_{C}+\gamma \cdot \mathbf{1}=\delta_{0} \cdot \mathbf{1}$, where $\gamma$ is the constant resulting from the identical parts of the columns. Notice that $0 \leq \gamma \leq \delta_{0}$, otherwise solutions could be constructed violating the inequality $\delta^{T} x \leq \delta_{0}$. So,

$$
\delta_{C}=\left(\delta_{0}-\gamma\right) \cdot M_{C}^{-1} \cdot \mathbf{1}
$$

Since $\delta_{C}$ is nonzero, it follows that $\delta_{0}-\gamma \neq 0$. Equalities (3.31) and (3.32) imply that $\delta_{i j}=\frac{\delta_{0}-\gamma}{\rho_{0}} \cdot \pi_{i j}$ for all $\{i, j\} \in E_{n}\left(V_{C}\right)$. This completes the proof. 
Notice that arguments used in the proof of Lemma 3.22 imply that for each facet of $P^{*}$ containing $F_{\pi}$, induced by some inequality $\omega^{T} x \leq \omega_{0}$, there exists a nonnegative coefficient $\varphi$ such that $\omega_{i j}=\varphi \cdot \pi_{i j}$ for all edges $\{i, j\} \in E_{n}\left(V_{C}\right)$.

The intersection patching procedure relies on the following condition.

Condition 3.23 Let $a^{T} x \leq a_{0}$ and $b^{T} x \leq b_{0}$ be two facet defining inequalities for $P\left(K_{n}\right)$, and let $V_{a}$ and $V_{b}$ be the node set of the supports of these inequalities. The following conditions must hold.

(i) $F_{a}:=\left\{x \in P\left(K_{n}\right) \mid a^{T} x=a_{0}\right\}$ and $F_{b}:=\left\{x \in P\left(K_{n}\right) \mid b^{T} x=b_{0}\right\}$ are different facets of $P\left(K_{n}\right)$.

(ii) $V_{a} \cap V_{b} \neq \varnothing$, and $V_{I}:=\left(V_{a} \cap V_{b}\right) \cup\left(V_{n} \backslash\left(V_{a} \cup V_{b}\right)\right)$.

(iii) $P^{*}$ is the face of $P\left(K_{n}\right)$ defined by the equalities $x_{i j}=0$ for all $\{i, j\} \in$ $E_{n}\left(V_{a} \backslash V_{l}, V_{b} \backslash V_{I}\right)$.

(iv) $a_{i j}=b_{i j}$ for all $\{i, j\} \in E_{n}\left(V_{I}\right)$.

(v) $\operatorname{cov}\left(a, V_{I}\right)=\operatorname{cov}\left(b, V_{I}\right)$.

(vi) $\operatorname{cov}\left(a, V_{0}\right)+\operatorname{cov}\left(b, V_{I} \backslash V_{0}\right) \geq \operatorname{cov}\left(a, V_{I}\right)$ for all $V_{0} \subseteq V_{I}$.

Theorem 3.24 (Intersection patching)

Let $a^{T} x \leq a_{0}$ and $b^{T} x \leq b_{0}$ be two facet defining inequalities for $P\left(K_{n}\right)$ satisfying Condition 3.23 . The inequality

$$
\sum_{\{i, j\} \in E_{n}\left(V_{a}\right)} a_{i j} x_{i j}+\sum_{\{i, j\} \in E_{n}\left(V_{b}, V_{b} \backslash V_{I}\right)} b_{i j} x_{i j} \leq a_{0}+b_{0}-\operatorname{cov}\left(a, V_{I}\right)
$$

defines a facet of $P^{*}$ unless $a_{i j}=b_{i j}=0$ for all $\{i, j\} \in E_{n}\left(V_{I}\right)$ and $\operatorname{cov}\left(a, V_{I}\right)=$ $\operatorname{cov}\left(b, V_{I}\right)=0$.

Proof First we show that the new inequality is valid for $P^{*}$. Let $x$ be an arbitrary integer solution in $P^{*}$. Then it is possible to partition $V_{I}$ into two sets $V_{I}(a)$ and $V_{I}(b)$, such that every node $b \in V_{I}$ that is in the same clique as a node in $V_{b} \backslash V_{I}$, is in $V_{I}(b)$, and all other nodes in $V_{I}$ are in $V_{I}(a)$. Then we have, using assumptions (v), (vi) and inequality (3.29):

$$
\sum_{\{i, j\} \in E_{n}\left(V_{a}\right)} a_{i j} x_{i j}+\sum_{\{i, j\} \in E_{n}\left(V_{b}, V_{b} \backslash V_{l}\right)} b_{i j} x_{i j}=
$$




$$
\begin{aligned}
& =\sum_{\left.\{i, j\} \in E_{n}\left(V_{a}, V_{a}\right\} V_{I}(b)\right)} a_{i j} x_{i j}+\sum_{\left.\{i, j\} \in E_{n}\left(V_{b}, V_{b}\right\} V_{I}(a)\right)} b_{i j} x_{i j} \\
& \leq a_{0}-\operatorname{cov}\left(a, V_{I}(b)\right)+b_{0}-\operatorname{cov}\left(b, V_{I}(a)\right) \\
& \leq a_{0}+b_{0}-\operatorname{cov}\left(a, V_{I}\right)
\end{aligned}
$$

This shows that inequality (3.33) is valid.

Now we show that it is facet defining. Let us invoke Lemma 3.22, where we set $E=E_{n}\left(V_{a} \backslash V_{I}, V_{b} \backslash V_{I}\right), V_{C}=V_{I} \cup V_{a}$, and where $\pi^{T} x \leq \pi_{0}$ is represented by inequality (3.33), and $\rho^{T} x \leq \rho_{0}$ is represented by $a^{T} x \leq a_{0}$. Lemma 3.22 implies that there exists a facet defined by the inequality $\delta^{T} x \leq \delta_{0}$, containing the face induced by inequality (3.33), such that

$$
\delta_{i j}=\alpha \cdot a_{i j} \quad \forall\{i, j\} \in E_{n}\left(V_{I} \cup V_{a}\right) \quad(\alpha>0)
$$

Now let us again apply Lemma 3.22 where we set $E=E_{n}\left(V_{a} \backslash V_{I}, V_{b} \backslash V_{I}\right)$, $V_{C}=V_{I} \cup V_{b}$, and where $\pi^{T} x \leq \pi_{0}$ is represented by inequality (3.33), and $\rho^{T} x \leq \rho_{0}$ is represented by $b^{T} x \leq b_{0}$. As discussed in the remark following the proof of Lemma 3.22, it follows that

$$
\delta_{i j}=\beta \cdot b_{i j} \quad \forall\{i, j\} \in E_{n}\left(V_{I} \cup V_{b}\right) \quad(\beta>0)
$$

We now distinguish two cases:

(i) There exists an $\{i, j\} \in E_{n}\left(V_{I}\right)$ such that $a_{i j} \neq 0$. We have: $\delta_{i j}=\alpha a_{i j}=$ $\beta b_{i j}$ for all $\{i, j\} \in E_{n}\left(V_{l}\right)$. Since $a_{i j}=b_{i j} \neq 0$, it follows that $\alpha=\beta>0$. Thus, the coefficients $\delta_{i j}$ equal the coefficients of inequality (3.33) up to multiplication with a strictly positive scalar which implies that inequality (3.33) is facet defining for $P^{*}$.

(ii) There exists no $\{i, j\} \in E\left(V_{I}\right)$ such that $a_{i j} \neq 0$. First, consider a solution satisfying both inequality (3.33) and $a^{T} x \leq a_{0}$ at equality. Such a solution exists by the definition of a covering. We derive, using equalities (3.31) and $(3.32)$

$$
\begin{aligned}
\delta_{0} & =\sum_{\{i, j\} \in E_{n}\{V)} \delta_{i j} x_{i j} \\
& =\sum_{\{i, j\} \in E_{n}\left(V_{I} \cup V_{a}\right)} \alpha \cdot a_{i j} x_{i j}+\sum_{\{i, j\} \in E_{n}\left(V_{b} \backslash V_{I}\right)} \beta \cdot b_{i j} x_{i j} \\
& =\alpha \cdot a_{0}+\beta \cdot\left(b_{0}-\operatorname{cov}\left(b, V_{I}\right)\right)
\end{aligned}
$$


Next, consider a solution satisfying both inequality (3.33) and inequality $b^{T} x \leq$ $b_{0}$ at equality. In a similar fashion we derive: $\delta_{0}=\alpha\left(a_{0}-\operatorname{cov}\left(a, V_{I}\right)\right)+$ $\beta b_{0}$. Combining these equalities, we obtain $\alpha \operatorname{cov}\left(a, V_{I}\right)=\beta \operatorname{cov}\left(b, V_{I}\right)$. If $\operatorname{cov}\left(a, V_{I}\right)>0$, it follows that $\alpha=\beta>0$. Thus, the coefficients $\delta_{i j}$ equal the coefficients of inequality (3.33) up to multiplication with a scalar which implies that inequality (3.33) is facet defining for $P^{*}$.

In case $\operatorname{cov}\left(a, V_{I}\right)=0$, the new inequality is just the sum of the two facet defining inequalities $a^{T} x \leq a_{0}$ and $b^{T} x \leq b_{0}$, and therefore not facet defining.

As described in Section 3.3, facet defining inequalities of $P^{*}$ can be lifted to facet defining inequalities of $P\left(K_{n}\right)$ by applying ordinary sequential lifting procedures.

Example 3.25 Consider the following 2-chorded odd cycle inequalities for an instance of clique partitioning on 7 nodes (see Figure 3.4 ).

$$
\begin{aligned}
& x_{12}+x_{23}+x_{34}+x_{45}+x_{51}-x_{13}-x_{24}-x_{35}-x_{41}-x_{52} \leq 2 \\
& x_{12}+x_{23}+x_{36}+x_{67}+x_{71}-x_{13}-x_{26}-x_{37}-x_{61}-x_{72} \leq 2
\end{aligned}
$$
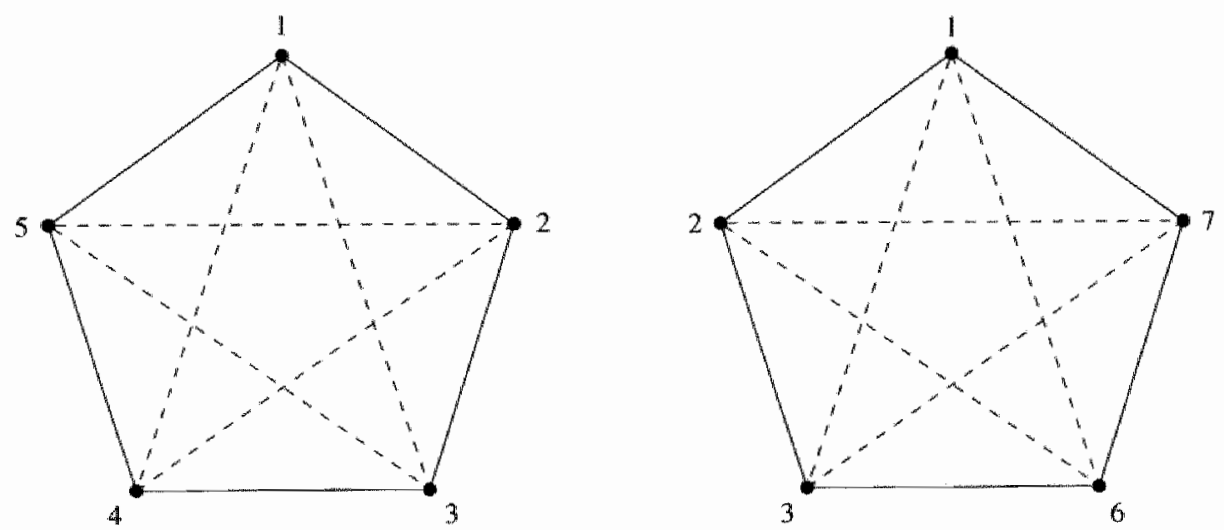

Figure 3.4: Two 2-chorded odd cycle inequalities. 
It follows that $V_{I}=\{1,2,3\}$ and $\operatorname{cov}\left(a, V_{I}\right)=1$. Notice that assumptions (i) -(vi) are satisfied. Thus, by Theorem 3.24 , the inequality

$$
\begin{aligned}
& x_{12}+x_{23}+x_{34}+x_{45}+x_{51}+x_{36}+x_{67}+x_{71}-x_{13}-x_{24} \\
& -x_{35}-x_{41}-x_{52}-x_{26}-x_{37}-x_{61}-x_{72} \leq 2+2-1=3
\end{aligned}
$$

defines a facet of $\left\{x \in P\left(K_{n}\right) \mid x_{57}=x_{56}=x_{47}=x_{46}=0\right\}$. By sequentially lifting the variables $x_{57}, x_{56}, x_{47}$ and $x_{46}$ (in that order!), we find the inequality (see Figure 3.5)

$$
\begin{aligned}
& x_{12}+x_{23}+x_{34}+x_{45}+x_{51}+x_{36}+x_{67}+x_{71}-x_{13}-x_{24} \\
& -x_{35}-x_{41}-x_{52}-x_{26}-x_{37}-x_{61}-x_{72}-x_{57} \leq 3
\end{aligned}
$$

which defines a facet of $P\left(K_{n}\right)$.

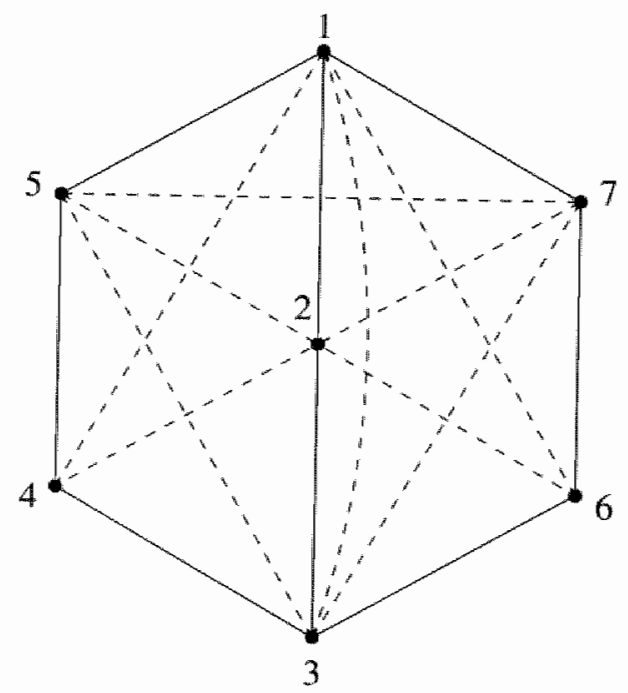

Figure 3.5: Patching of two odd-cycle inequalities.

It is not difficult to verify that the intersection patching procedure can be applied to any two odd-cycle inequalities such that the positive support of $V_{I}$ is a path with an odd number of nodes. 
Sometimes the derivation of a facet of $P\left(K_{n}\right)$ from a facet of $P^{*}$ is straight:forward, and an explicit formulation can be given. Consider the case of patching. two star inequalities.

\section{Corollary 3.26 (Star patching)}

Let $a^{T} x \leq 1$ be an $(S, T)$-inequality with $S=\{s\}$ and let $b^{T} x \leq c$ be a facet defining star inequality, such that their supports have only the center $s$ in common. Then the following inequality is facet defining:

$$
\sum_{\{i, j\} \in E(V)} \gamma_{i j} x_{i j} \leq c
$$

where

$$
\gamma_{i j}= \begin{cases}c \cdot a_{i j} & \text { if }\{i, j\} \in E_{n}\left(V_{a}\right) \\ b_{i j} & \text { if }\{i, j\} \in E_{n}\left(V_{b}\right) \\ -b_{j s} & \text { if }\{i, j\} \in E_{n}\left(V_{a} \backslash\{s\}, V_{b} \backslash\{s\}\right) \\ 0 & \text { otherwise }\end{cases}
$$

Proof Notice that for a star inequality the covering of the center equals the right-hand side. Applying Theorem 3.24 and next sequential lifting of the coefficients corresponding to the edges in $E_{n}\left(V_{a} \backslash\{s\}, V_{b} \backslash\{s\}\right)$ yields the corollary.

The disjoint patching procedure relies on the following condition.

Condition 3.27 Let $a^{T} x \leq a_{0}$ and $b^{T} x \leq b_{0}$ be two facet defining inequalities for $P\left(K_{n}\right)$, and let $V_{a}$ and $V_{b}$ be the node set of the supports of these inequalities. The following conditions must hold.

(i) $F_{a}:=\left\{x \in P\left(K_{n}\right) \mid a^{T} x=a_{0}\right\}$ and $F_{b}:=\left\{x \in P\left(K_{n}\right) \mid b^{T} x=b_{0}\right\}$ are different facets of $P\left(K_{n}\right)$.

(ii) $V_{a} \cap V_{b}=\varnothing$.

(iii) $q, r, s \in V_{a}$ are three different nodes in $V_{a}$.

(iv) $t, u, v \in V_{b}$ are three different nodes in $V_{b}$.

(v) $P^{*}$ is the face of $P\left(K_{n}\right)$ defined by the equalities $x_{i j}=0$ for all $\{i, j\} \in$ $E_{n}\left(V_{\alpha}, V_{b}\right) \backslash E_{n}(\{q, r, s\},\{t, u, v\})$. 
(vi) $\gamma_{s t}:=\operatorname{cov}(a,\{s\})=\operatorname{cov}(b,\{t\})$.

To patch the two inequalities we are looking for values of the coefficients $\gamma_{i j}$ such that the following inequality defines a facet of $P^{*}$.

$$
(a+b)^{T} x+\sum_{\{i, j\} \in E\left(V_{a}^{*}, v_{b}\right\}} \gamma_{i j} x_{i j} \leq a_{0}+b_{0}
$$

In the next two theorems we give two options for the values of the coefficients $\gamma_{i j}$.

\section{Theorem 3.28 (Disjoint patching I)}

Let $a^{T} x \leq a_{0}$ and $b^{T} x \leq b_{0}$ be two facet defining inequalities for $P\left(K_{n}\right)$ satisfying Condition 3.27. Assume $\operatorname{cov}(a,\{q\})=\operatorname{cov}(a,\{r\})=\operatorname{cov}(b,\{u\})=$ $\operatorname{cov}(b,\{v\})=0, \operatorname{cov}(a,\{q, s\})=a_{q s}, \operatorname{cov}(a,\{r, s\})=a_{r s}, \operatorname{cov}(b,\{u, t\})=b_{u t}$, and $\operatorname{cov}(b,\{v, t\})=b_{v t}$. If $\gamma_{s t}>0$ the following inequality defines a facet of $P^{*}$ :

$$
(a+b)^{T} x+\gamma_{s t}\left(x_{s t}-x_{s u}-x_{r t}-x_{q v}\right) \leq a_{0}+b_{0}
$$

Proof First we show that inequality (3.34) is valid for $P^{*}$. Let $x$ be an arbitrary integer solution in $P^{*}$. If $s$ and $t$ are not in the same clique, it follows that $x_{s t}=0$ and inequality (3.34) is satisfied by validity of the two original inequalities. So, we may assume $x_{s t}=1$. Notice that, since $x$ is in $P^{*}$, no node in $V_{a} \backslash\{q, r, s\}\left(V_{b} \backslash\{t, u, v\}\right)$ can be in a clique with $t(s)$. Now, if neither $q$ nor $r$ are in the same clique as $s$ and $t$, we have $a^{T} x \leq a_{0}-\operatorname{cov}(a,\{s\})=a_{0}-\gamma_{s t}$ and therefore inequality (3.34) is satisfied. Similarly, if neither $u$ nor $v$ are in the same clique as $s$ and $t$, we have $b^{T} x \leq b_{0}-\gamma_{s t}$ and inequality (3.34) is satisfied. So, at least one of the vertices $q$ and $r$ belong to the same clique as $s$ and $t$. And similarly, at least one of the vertices $u$ and $v$ belong to the same clique as $s$ and $t$. Now, if $r(u)$ is in the same clique as $s$ and $t$, then $x_{r t}=1$ $\left(x_{t s s}=1\right.$ ) and inequality (3.34) is satisfied. Otherwise, $q$ and $v$ are in the same clique as $s$ and $t$, but then $x_{q v}=1$ and inequality (3.34) is satisfied.

Now we show that it is facet defining. Let us invoke Lemma 3.22 , where we set $E=E_{n}\left(V_{a}, V_{b}\right) \backslash E_{n}(\{q, r, s\},\{t, u, v\}), V_{C}=V_{a}$, and where $\pi^{T} x \leq \pi_{0}$ is 
represented by inequality (3.34), and $\rho^{T} x \leq \rho_{0}$ is represented by $a^{T} x \leq a_{0}$. Lemma 3.22 implies that there exists a facet defined by the inequality $\delta^{T} x \leq$ $\delta_{0}$, containing the face induced by inequality (3.34), such that

$$
\delta_{i j}=\alpha \cdot a_{i j} \quad \forall\{i, j\} \in E_{n}\left(V_{a}\right) \quad(\alpha>0)
$$

Set $E=E_{n}\left(V_{a}, V_{b}\right) \backslash E_{n}(\{q, r, s\},\{t, u, v\}), V_{C}=V_{b}$, and apply Lemma 3.22 , where $\pi^{T} x \leq \pi_{0}$ is represented by inequality (3.34), and $\rho^{T} x \leq \rho_{0}$ is represented by $b^{T} x \leq b_{0}$. As discussed in the remark following the proof of Lemma 3.22 , it follows that

$$
\delta_{i j}=\beta \cdot b_{i j} \forall\{i, j\} \in E_{n}\left(V_{b}\right) \quad(\beta \geq 0)
$$

Summarizing, there exists a facet of $P^{*}$ containing the face of $P^{*}$ defined by inequality (3.34), which is induced by an inequality of the form:

$$
(\alpha a+\beta b)^{T} x+\sum_{\{i, j\} \in E_{n}\left(V_{a}, V_{b}\right)} \delta_{i j} x_{i j} \leq \alpha a_{0}+\beta b_{0}
$$

Let us now show that $\alpha=\beta$ and $\delta_{i j}=\alpha \cdot \gamma_{i j}$.

First, consider $\delta_{r u}$. Since $\operatorname{cov}(a,\{r\})=\operatorname{cov}(b,\{u\})=0$ there is a solution $x$ such that $a^{T} x=a_{0}, b^{T} x=b_{0}$, and $\{r\}$ and $\{u\}$ are cliques. By changing $\{r\}$ and $\{u\}$ to one clique $\{r, u\}$, we obtain a new solution $y$ satisfying the new inequality at equality. Comparing $x$ and $y$ yields $\delta_{r u}=0=\gamma_{r u}$. Analogously, we derive $\delta_{r v}=0=\gamma_{r v}$ and $\delta_{q u}=0=\gamma_{q u}$.

Next, consider $\delta_{q t}$. Since $\operatorname{cov}(a,\{q\})=0$ and $\operatorname{cov}(b,\{u, t\})=b_{u t}$ there is a solution $x$ such that $a^{T} x=a_{0}, b^{T} x=b_{0}$, and $\{q\}$ and $\{u, t\}$ are cliques. By changing $\{q\}$ and $\{u, t\}$ to one clique $\{q, u, t\}$ we obtain a new solution $y$ satisfying the new inequality at equality. Comparing $x$ and $y$ we conclude that $\delta_{q t}+\delta_{q u}=0$. Since $\delta_{q u}=0$, it follows that $\delta_{q t}=0=\gamma_{q t}$. In an analogue way, one can show that $\delta_{s v}=0=\gamma_{s v}$.

Third, consider $\delta_{s t}$. Since $\operatorname{cov}(a,\{q, s\})=a_{q s}$, and by the definition of a covering, there is a solution $x$ such that $a^{T} x=a_{0}, b^{T} x=b_{0}-\operatorname{cov}(b,\{t\})$, and $\{q, s\}$ and $\{t\}$ are cliques. By changing $\{q, s\}$ and $\{t\}$ to one clique $\{q, s, t\}$ we obtain a new solution $y$ satisfying the new inequality at equality. Comparing 
$x$ and $y$ we conclude that $\delta_{s t}=\beta \operatorname{cov}(b,\{t\})=\beta \gamma_{s t}$. In a similar way, one can derive $\delta_{s t}=\alpha \operatorname{cov}(a,\{s\})=\alpha \gamma_{s t}$. Using (3.34) and (3.32), it follows that $\alpha=\beta$.

Finally, consider the negative coefficients. Since $\operatorname{cov}(a,\{q, s\})=a_{q s}$, and $\operatorname{cov}(b,\{u, t\})=b_{u t}$, there is a solution $x$ such that $a^{T} x=a_{0}, b^{T} x=b_{0}$. and $\{q, s\}$ and $\{u, t\}$ are cliques. By changing $\{q, s\}$ and $\{u, t\}$ to one clique $\{q, s, u, t\}$ we obtain a new solution $y$ satisfying the new inequality at equality. Comparing $x$ and $y$ we have $\delta_{q u}+\delta_{q t}+\delta_{s u}+\delta_{s t}=0$. Substituting the earlier results $\delta_{q u}=\delta_{q t}=0$ it follows that $\delta_{s u}=-\delta_{s t}$. In an analogue way, one can show $\delta_{r t}=-\delta_{s t}$, and $\delta_{q v}=-\delta_{s t}$.

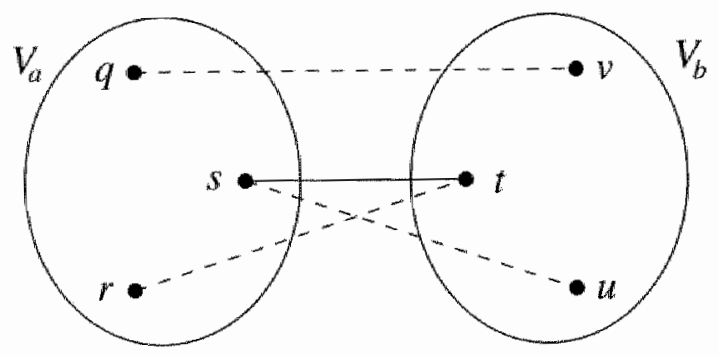

Figure 3.6: Support of an inequality obtained from disjoint patching I.

Another possibility for the $\gamma$-coefficients is as follows.

Theorem 3.29 (Disjoint patching II)

Assume that $\operatorname{cov}(a,\{q\})=\operatorname{cov}(a,\{r\})=\operatorname{cov}(b,\{u\})=\operatorname{cov}(b,\{v\})=0$, $\operatorname{cov}(a,\{q, s\})=a_{q s}, \operatorname{cov}(a,\{r, s\})=a_{r s}, \operatorname{cov}(a,\{q, r, s\})=a_{q s}+a_{r s}+a_{q r}$, $\operatorname{cov}(b,\{u, t\})=b_{u t}, \operatorname{cov}(b,\{v, t\})=b_{v t}$, and $\operatorname{cov}(b,\{u, v, t\})=b_{u t}+b_{v t}+b_{u v}$. If $\gamma_{s t}>0$ the following inequality defines a facet of $P^{*}$ :

$$
(a+b)^{T} x+\gamma_{s t}\left(x_{s t}-x_{q u}-x_{q v}-x_{r u}-x_{\tau v}\right) \leq a_{0}+b_{0}
$$

Proof First we show that the inequality is valid for $P^{*}$. Let $x$ be an arbitrary integer solution in $P^{*}$. If $s$ and $t$ are not in the same clique, the inequality is satisfied by validity of the two original inequalities. So, we may assume $x_{s t}=1$. If neither $q$ nor $r$ are in the same clique as $s$ and $t$, we have $a^{T} x \leq a_{0}-\gamma_{s t}$ and therefore our inequality is satisfied. Similarly, if neither $u$ nor $v$ are in the 
same clique as $s$ and $t$, we have $b^{T} x \leq b_{0}-\gamma_{s t}$ and our inequality is satisfied. So either $q$ or $r$ or both and either $u$ or $v$ or both are in the same clique as $s$ and $t$. This implies $\left(x_{s t}-x_{q u}-x_{q v}-x_{r u}-x_{r v}\right) \leq 0$ and therefore the inequality is satisfied by validity of the two original inequalities.

Now we show that it is facet defining. Let us invoke Lemma 3.22, where we set $E=E_{n}\left(V_{a}, V_{b}\right) \backslash E_{n}(\{q, r, s\},\{t, u, v\}), V_{C}=V_{a}$, and where $\pi^{T} x \leq \pi_{0}$ is represented by inequality (3.34), and $\rho^{T} x \leq \rho_{0}$ is represented by $a^{T} x \leq a_{0}$. Lemma 3.22 implies that there exists a facet defined by the inequality $\delta^{T} x \leq$ $\delta_{0}$, containing the face induced by inequality (3.34), such that

$$
\delta_{i j}=\alpha \cdot a_{i j} \quad \forall\{i, j\} \in E_{n}\left(V_{a}\right) \quad(\alpha>0)
$$

Set $E=E_{n}\left(V_{a}, V_{b}\right) \backslash E_{n}(\{q, r, s\},\{t, u, v\}), V_{C}=V_{b}$, and apply Lemma 3.22, where $\pi^{T} x \leq \pi_{0}$ is represented by inequality (3.34), and $\rho^{T} x \leq \rho_{0}$ is represented by $b^{T} x \leq b_{0}$. As discussed in the remark following the proof of Lemma 3.22 , it follows that

$$
\delta_{i j}=\beta \cdot b_{i j} \quad \forall\{i, j\} \in E_{n}\left(V_{b}\right) \quad(\beta>0)
$$

Summarizing, we have that there is a facet of $P^{*}$ containing the face of $P^{*}$ defined by the inequality, which is defined by an inequality of the form:

$$
(\alpha a+\beta b)^{T} x+\sum_{\{i, j\} \in E\left(V_{a}, V_{b}\right)} \delta_{i j} x_{i j} \leq \alpha a_{0}+\beta b_{0}
$$

What is left to be shown is that $\alpha=\beta$ and $\delta_{i j}=\alpha \cdot \gamma_{i j}$.

First, consider $\delta_{\tau u}$. Since $\operatorname{cov}(a,\{r\})=\operatorname{cov}(b,\{u\})=0$ there is a solution $x$ such that $a^{T} x=a_{0}, b^{T} x=b_{0}$, and $\{r\}$ and $\{u\}$ are clliques. By changing $\{r\}$ and $\{u\}$ to one clique $\{r, u\}$ we obtain a new solution $y$ satisfying the new inequality at equality. Comparing $x$ and $y$ we conclude $\delta_{r u}=0=\gamma_{r u}$. Analogously, we derive $\delta_{r v}=0=\gamma_{r v}$ and $\delta_{q u}=0=\gamma_{q u}$.

Next, consider the coefficients involving $t$, that is $\delta_{q t}, \delta_{r t}$, and $\delta_{s t}$. Since $\operatorname{cov}(a,\{q, s\})=a_{q s}$, and by the definition of covering there is a solution $x$ such that $a^{T} x=a_{0}, b^{T} x=b_{0}-\operatorname{cov}(b,\{t\})$, and $\{q, s, t\}$ is a clique. Since $\operatorname{cov}(a,\{r, s\})=a_{r s}$, and by the definition of covering there is a solution $y$ 
such that $a^{T} y=a_{0}, b^{T} y=b_{0}-\operatorname{cov}(b,\{t\})$, and $\{r, s, t\}$ is a clique. Since $\operatorname{cov}(\alpha,\{q, r, s\})=a_{q s}+a_{r s}+a_{q r}$, and by the definition of covering there is a solution $z$ such that $a^{T} z=a_{0}, b^{T} z=b_{0}-\operatorname{cov}(b,\{t\})$, and $\left\{q, r_{n} s, t\right\}$ is a clique.

Comparing $x, y$, and $z$ we conclude $\delta_{q t}=0=\gamma_{q t}, \delta_{r t}=0=\gamma_{r t}$, and $\delta_{s t}=$ $\beta \operatorname{cov}(b,\{t\})=\beta \gamma_{s t}$, In an analogue way, one can show $\delta_{s u}=0=\gamma_{s u}, \delta_{s v}=$ $0=\gamma_{s v}$, and $\delta_{s t}=\alpha \operatorname{cov}(\alpha,\{s\})=\alpha \gamma_{s t}$. From these results it immediately follows that $\alpha=\beta$.

Finally, consider the negative coefficients. Since $\operatorname{cov}(a,\{q, s\})=a_{q s}$, and $\operatorname{cov}(b,\{u, t\})=b_{u t}$, there is a solution $x$ such that $a^{T} x=a_{0}, b^{T} x=b_{0}$, the new inequality is satisfied at equality, and $\{q, s\}$ and $\{u, t\}$ are cliques. By changing $\{q, s\}$ and $\{u, t\}$ to one clique $\{q, s, u, t\}$ we obtain a new solution $y$ satisfying the new inequality at equality. Comparing $x$ and $y$ we have $\delta_{q u}+\delta_{q t}+\delta_{s u}+\delta_{s t}=$ 0 . Substituting the earlier results $\delta_{q t}=\delta_{s u}=0$ we conclude $\delta_{q u}=-\delta_{s t}$. In an analogue way, one can show $\delta_{q v}=-\delta_{s t}, \delta_{r u}=-\delta_{s t}$, and $\delta_{r v}=-\delta_{s t}$.

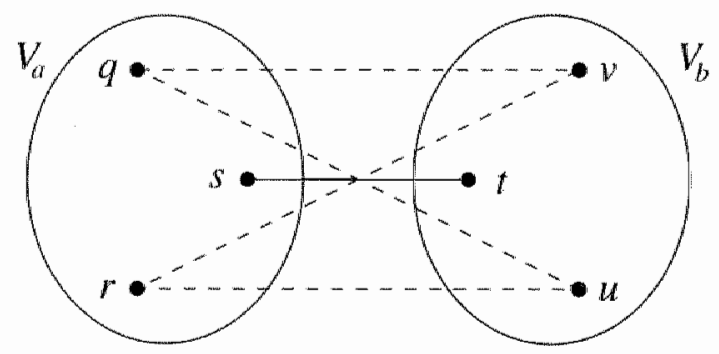

Figure 3.7: Support of an inequality obtained from disjoint patching II.

Starting with the results of Theorem 3.28 and Theorem 3.29, we can derive ficcts of the full dimensional polytope $P\left(K_{n}\right)$ by applying ordinary sequential. lifting procedures. In some cases it is possible to derive an explicit formulation of inequalities resulting from a disjoint patching procedure. For example, when both inequalities are $(S, T)$-inequalities with $|S|=1$ :

\section{Theorem 3.30 (Disjoint (S,T)-patching)}

Let $a^{T} x \leq 1$ and $b^{T} x \leq 1$ be $(S, T)$-inequalities, both with right-hand side 1 , and with disjoint supports on $V_{a}$ respectively $V_{b}$. Let $s(t)$ be the center of $a^{T} x \leq 1\left(b^{T} x \leq 1\right)$. Let $V_{a}=U_{a} \cup W_{a} \cup\{s\}$ such that these three sets are disjoint. Let $V_{b}=U_{b} \cup W_{b} \cup\{t\}$ such that these three sets are disjoint. Then 
the following inequality is valid. It is facet defining if $U_{a}$ and $U_{b}$ are either both empty or both nonempty, and if $W_{a}$ and $W_{b}$ are both nonempty:

$$
(a+b)^{T} x+x_{s t}-\sum_{j \in U_{b}} x_{s j}-\sum_{i \in U_{a}} x_{i t}-\sum_{\{i, j\} \in E_{n}\left(W_{a}, W_{b}\right)} x_{i j} \leq 2
$$

Proof First we show that the inequality is valid for $P\left(K_{n}\right)$. Let $x$ be an arbitrary integer solution in $P\left(K_{n}\right)$. If $s$ and $t$ are not in the same clique, the inequality is satisfied by validity of the two original inequalities. So, we may assume $x_{s t}=1$. If there are no other nodes in $V_{a}$ in the same clique as $s$ and $t$, we have $a^{T} x \leq 0$ and therefore inequality (3.36) is satisfied. Similarly, if there are no other nodes in $V_{b}$ in the same clique as $s$ and $t$, we have $b^{T} x \leq 0$ and inequality (3.36) is satisfied. So there is at least one node $r \in V_{a}$ and at least one node $u \in V_{b}$ which are in the same clique as $s$ and $t$. Then $r \in U_{a}$, $u \in U_{b}$, or $\{r, u\} \in E_{n}\left(W_{a}, W_{b}\right)$, implying

$$
x_{s t}-\sum_{j \in U_{b}} x_{s j}-\sum_{i \in U_{a}} x_{i t}-\sum_{\{i, j\} \in E_{n}\left(W_{\alpha}, W_{b}\right)} x_{i j} \leq 0
$$

and therefore the inequality is satisfied by validity of the two original inequalities.

Next we need to show that the inequality defines a facet of $P\left(K_{n}\right)$. In case $U_{a}$ and $U_{b}$ are both nonempty from Theorem 3.28 , and in case $U_{a}$ and $U_{b}$ are both empty from Theorem 3.29 , we can deduce that there is a facet of $P\left(K_{n}\right)$ containing the face of $P\left(K_{n}\right)$ defined by the inequality, which is defined by an inequality of the form

$$
\alpha(a+b)^{T} x+\sum_{\{i, j\} \in E_{n}\left(V_{a}, V_{b}\right)} \delta_{i j} x_{i j} \leq 2 \alpha
$$

where $\delta_{g t}$ equals $\alpha$. It remains to show that $\delta_{i j}=\alpha \cdot \gamma_{i j}$ for all edges $\{i, j\} \in$ $E_{n}\left(V_{a}, V_{b}\right)$.

First, consider $\delta_{i j}$ for all $\{i, j\} \in E_{n}\left(V_{a}, V_{b}\right) \backslash E_{n}\left(W_{a}, W_{b}\right)$. Since cov $(a,\{i\})=$ $\operatorname{cov}(b,\{j\})=0$, there is a solution $x$ such that $a^{T} x=1, b^{T} x=1$, the new inequality is satisfied at equality, and $\{i\}$ and $\{j\}$ are cliques. Merging $\{i\}$ and 
$\{j\}$ to one clique $\{i, j\}$ yields a new solution $y$ satisfying the new inequality at equality. Comparing $x$ and $y$ we conclude $\delta_{i j}=0=\gamma_{i j}$.

Next, consider $\delta_{s j}$ for $j \in W_{b}$. There exists a solution $x$ such that $a^{T} x=0$, $b^{T} x=1$, the new inequality is satisfied at equality, and $\{s, t, j\}$ is a clique. Then $x$ satisfies the patching at equality, yielding: $\delta_{s t}+\delta_{t j}+\delta_{s j}=2 \alpha$. Plugging in the earlier results $\delta_{s t}=\alpha$ and $\delta_{i j}=\alpha$ gives $\delta_{s j}=0=\gamma_{s j}$.

In a similar way it is argued that $\delta_{i t}=0=\gamma_{i t}$ for all $i \in W_{a}$.

Now, consider $\delta_{s j}$ for $j \in U_{b}$. There exists a solution $x$ such that $a^{T} x=1$, $b^{T} x=1,\{i, s, t, j\}$ is a clique for a $i \in W_{a}$ (which exists since $W_{a} \neq \varnothing$ ) and $x$ satisfies the patching at equality, yielding: $\delta_{s t}+\delta_{t j}+\delta_{s j}+\delta_{i j}=0$. Plugging in the earlier results $\delta_{s t}=\alpha, \delta_{i j}=0$, and $\delta_{i t}=0$ gives $\delta_{s j}=-\alpha=\alpha \gamma_{s j}$.

In a similar way it is argued that $\delta_{i \ell}=-\alpha=\alpha \gamma_{i t}$ for all $i \in U_{a}$.

Finally, consider $\delta_{i j}$ for $\{i, j\} \in E\left(W_{a}, W_{b}\right)$. There exists a solution $x$ such that: $a^{T} x=1, b^{T} x=1,\{\hat{i}, s, t, j\}$ is a clique, and satisfies the patching at equality. This yields: $\delta_{s t}+\delta_{t j}+\delta_{s j}+\delta_{i j}=0$. Plugging in the earlier results $\delta_{s t}=\alpha, \delta_{s j}=0$, and $\delta_{i t}=0$ gives $\delta_{i j}=-\alpha=\alpha \gamma_{i j}$.

We refer to inequalities (3.36) as composed 2-partition inequalities.
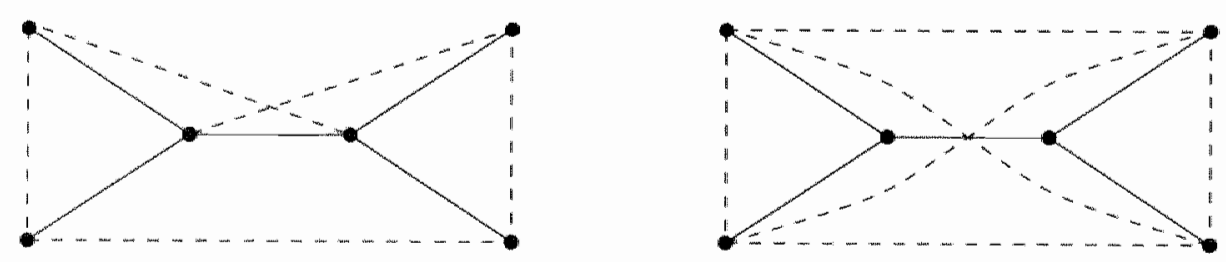

Figure 3.8: Disjoint (S, $T)$-patching of two triangle inequalities.

\subsection{Facets with right-hand side 1 or 2}

In this section we show that for $P\left(K_{n}\right)$ all facet defining inequalities $a^{T} x \leq a_{0}$ with integer coefficients and $a_{0} \in\{1,2\}$ are known. 


\subsubsection{Right-hand side 1}

In the previous chapter we described the so-called 2-partition inequalities. The following theorem proves that these inequalities are the only facet defining inequalities for the clique partitioning polytope with integer coefficients and right-hand side 1 .

Theorem 3.31 Let $K_{n}=\left(V_{n,}, E_{n}\right)$ be the complete graph on $n$ vertices. Any facet defining inequality $a^{T} x \leq 1\left(a \in \mathbb{Z}^{\left|E_{n 2}\right|}\right)$ for $P\left(K_{n}\right)$ is a 2-partition inequality.

Proof Obviously, $a_{i j} \leq 1$ for all $\{i, j\} \in E_{n}$. If there is only one edge $\{i, j\} \in E_{n}$ with $a_{i j}=1$, then $a^{T} x \leq 1$ is trivially implied by $x_{i j} \leq 1$.

If $F:=\left\{\{i, j\} \in E_{n} \mid a_{i j}=1\right\}$ contains at least 2 edges, then all edges in $F$ are incident to a single vertex $s \in V_{n}$ (otherwise one can exhibit a clique partition $M \subseteq E_{n}$ with $a^{T} \chi^{M}>1$ ). Consider two edges $\{s, i\},\{s, j\} \in F$. Since $E_{n}(\{s, i, j\})$ is a feasible clique partition, it follows that $a_{i, j} \leq-1$. It is now easy to observe that $a^{T} x \leq 1$ is an $(S, T)$-inequality with $S=\{s\}$, since otherwise it, would be implied by one.

\subsubsection{Right-hand side 2}

In order to characterize all facets with right-hand side 2, we first show that the positive support of any facet defining inequality is connected.

Lemma 3.32 Let $K_{n}=\left(V_{n}, E_{n}\right)$ be the complete graph on $n$ vertices, and let $a^{T} x \leq a_{0}$ be a facet defining inequality for $P\left(K_{n}\right)$. The graph $H=\left(V_{n}(F), F\right)$ induced by $F:=\left\{\{i, j\} \in E_{n} \mid a_{i j}>0\right\}$ is connected.

Proof Suppose $H$ is not connected, but instead consists of $k \geq 2$ components. Let $W_{i} \subseteq V_{n}(F)$ be the set of vertices of component $i=1, \ldots, k$. Since the support of $a$ is connected [39], there exist two components with vertex sets $W_{i}$ and $W_{j}(1 \leq i<j \leq k)$, and an edge $\left\{u_{i}, u_{j}\right\} \in E_{n i}$ with $a_{u_{i}, u_{j}}<0$ connecting 
a vertex $u_{i} \in W_{i}$ to a vertex $u_{j} \in W_{j}$. Consider any clique partition $A \subseteq E_{n}$ containing this edge, and let

$$
B:=A \cap\left(\bigcup_{i=1}^{k} E_{n}\left(W_{i}\right)\right)
$$

Then, on the one hand all edges $\{i, j\} \in A$ with $a_{i j}>0$ belong to $B$, but on the other hand $\left\{u_{i}, u_{j}\right\} \notin B$, hence

$$
a^{T} \chi^{A}<a^{T} \chi^{B} \leq a_{0}
$$

So, the face induced by $a^{T} x=a_{0}$ is contained in the face induced by $x_{u_{i}, u_{j}}=0$, a contradiction. Thus, $H$ is connected.

We can now classify all facets of $P\left(K_{n}\right)$ with right-hand side 2 .

Theorem 3.33 Let $K_{n}=\left(V_{n}, E_{n}\right)$ be the complete graph on $n$ vertices. All facet defining inequalities of $P\left(K_{n}\right)$ with right-hand side 2 belong to one of the following classes.

(i) 2-partition inequalities (see inequalities (3.1)),

(ii) 2-chorded cycles of length 5 (see inequalities (3.2)),

(iii) Inequalities obtained by applying intersection patching to two 2-partition inequalities with right-hand side 1 (see inequalities (3.28) with $\left|S_{1}\right|=$ $\left.\left|S_{2}\right|=1\right)$

(iv) (Lifted) stable set inequalities (see inequalities (3.12) and (3.27) with $\alpha(H)=2$ ),

(v) Composed 2-partition inequalities (3.36).

Proof First, observe that the 2-chorded path inequalities with right-hand side 2 are contained in class (iii).

Now, let $a^{T} x \leq 2$ be a facet defining inequality for $P\left(K_{n}\right)$. It is obvious that $a_{i j} \leq 2$ for all $\{i, j\} \in E_{n}$. We distinguish the following three cases.

(i) There are at least two edges $\{i, j\} \in E_{n}$ with $a_{i j}=2$, 
(ii) There is exactly one edge $\{i, j\} \in E_{n}$ with $a_{i j}=2$,

(iii) There are no edges $\{i, j\} \in E_{n}$ with $a_{i j}=2$.

(i) All edges $\{i, j\} \in E_{n}$ with $a_{i j}=2$ are incident to a common vertex, say vertex $s \in V_{n}$. Moreover, all edges $\{i, j\} \in E_{n}$ with $a_{i j}=1$ are also incident to $s$. For any two edges $\{s, i\}$ and $\{s, j\}$ with $a_{s i}=a_{s j}=2$ we have that $a_{i j} \leq-2$. If $a_{s i}=2$ and $a_{s j}=1$, then $a_{i j} \leq-1$. Define

$$
\begin{aligned}
& T=\left\{i \in V_{n} \mid a_{s i}=1\right\} \\
& F=\left\{\{i, j\} \in E_{n} \mid i, j \in T, a_{i j} \leq-1\right\}
\end{aligned}
$$

The graph $H=(T, F)$ has $\alpha(H) \leq 2$, since otherwise the inequality $a^{T} x \leq 2$ would not be valid. Hence, $a^{T} x \leq 2$ is a lifted stable set inequality.

(ii) Let $\{s, t\} \in E_{n}$ be the edge with $a_{s t}=2$. Then all edges $\{i, j\} \in E_{n}$ with $a_{i j}=1$ are either incident to $s$ or to $t$. Moreover, if $a_{s i}=1$, then $a_{t i i} \leq-1$, and if $a_{t i}=1$, then $a_{s i} \leq-1$. If there are no edges with coefficient equal to 1 incident to $s$ (or to $t$ ), then we have the same situation as in case (i). Otherwise, define

$$
\begin{aligned}
& S=\left\{i \in V_{n} \mid a_{s i}=1\right\} \\
& T=\left\{i \in V_{n} \mid a_{t i}=1\right\}
\end{aligned}
$$

where both $S$ and $T$ are nonempty. If $i_{y}, j \in S$ then $a_{i j} \leq-1$, and also if $i, j \in T$ we have that $a_{i j} \leq-1$. Hence, the inequality $a^{T} x \leq 2$ is implied by the sum of the two 2-partition inequalities

$$
\begin{aligned}
& x\left(E_{n}(\{s\}, S \cup\{t\})\right)-x\left(E_{n}(S \cup\{t\})\right) \leq 1 \\
& x\left(E_{n}(\{t\}, T \cup\{s\})\right)-x\left(E_{n}(T \cup\{s\})\right) \leq 1
\end{aligned}
$$

and therefore it is not facet defining.

(iii) All edges $\{i, j\} \in E_{n}$ have $a_{i j} \leq 1$. Let $G=\left(V_{n}(F), F\right)$ be the graph induced by $F:=\left\{\{i, j\} \in E_{n} \mid a_{i j}=1\right\}$. From Lemma 3.32 we know that $G$ is connected, so it contains a path of length at least 2. On the other hand, $a^{T} x \leq 2$ is a valid inequality, so $G$ cannot contain a path of length 5 or more. We distinguish three cases, depending on the length of a longest path in $G$. 
(iii.a) A longest path in $G$ has length 2. All edges $\{i, j\} \in E_{n}$ with $a_{i j}=1$. are incident to the same node, say node $s \in V_{n}$. Define $T$ and $F$ as in case (i), then again the graph $H=(T, F)$ has $\alpha(H) \leq 2$. Hence, the inequality $a^{T} x \leq 2$ is implied by a stable set inequality.

(iii.b) Let $\left\{v_{1}, \ldots, v_{4}\right\} \subseteq V_{n}$, and let $P:=\left\{\left\{v_{i}, v_{i+1}\right\} \| i=1,2,3\right\}$ be a longest path of length 3 , where $a_{i j}=1$ for all $\{i, j\} \in P$. If $a_{v_{1}, w_{4}}=1$, then there are no other edges $\{i, j\} \in E_{n}$ with $a_{i j}=1$, since otherwise $P$ is not a longest path in $G$. Moreover, $a_{v_{1}, v_{3}}+a_{v_{2}, v_{4}} \leq-2$. If $a_{v_{1}, v_{3}} \leq-2$ or $a_{v_{2}, v_{4}} \leq-2$, then $a^{T} x \leq 2$ is implied by two triangle inequalities. If $a_{v_{1}, v_{3}}=a_{v_{2}, v_{4}}=-1$, then $a^{T} x \leq 2$ is implied by an $(S, T)$-inequality with $S=\left\{v_{1}, v_{3}\right\}$ and $T=\left\{v_{2}, v_{3}\right\}$.

If $a_{v_{1}, v_{4}} \leq 0$, then all edges $\{i, j\} \in E_{n} \backslash P$ with $a_{i j}=1$ are either incident to $v_{2}$ or to $v_{3}$. Let $T_{1}:=\left\{i \in V_{n} \mid a_{v_{2}, i}=1\right\}$ and $T_{2}:=\left\{i \in V_{n} \mid a_{v_{3}, i}=1\right\}$ (hence, $v_{1} \in T_{1}$ and $v_{4} \in T_{2}$ ). Then $T_{1} \cap T_{2}=\varnothing$, otherwise $a^{T} x \leq 2$ would not be valid. This implies that the inequality $a^{T} x \leq 2$ can be obtained by applying disjoint $(S, T)$-patching to two $(S, T)$-inequalities, one with $S=\left\{v_{2}\right\}$ and $T=T_{1}$, and the other with $S=\left\{v_{3}\right\}$ and $T=T_{2}$.

(iii.c) Let $\left\{v_{1}, \ldots, v_{5}\right\} \subseteq V_{n}$, and let $P:=\left\{\left\{v_{i}, v_{i+1}\right\} \mid i=1, \ldots, 4\right\}$ be a longest path of length 4 , where $a_{i j}=1$ for all $\{i, j\} \in P$. Observe that $a_{v_{1}, v_{3}} \leq-1$, and also $a_{v_{3}, v_{5}} \leq-1$. If $a_{v_{1}, v_{5}}=1$, then we have a cycle of length 5 in $G$. All 2-chords of this cycle have coefficients at most -1 , and there are no other edges with a positive coefficient. Hence, the inequality $a^{T} x \leq 2$ is implied by a 2 -chorded cycle inequality.

We are left with the situation where $a_{v_{1}, w_{5}} \leq 0$ (see Figure 3.9). All edges $\{i, j\} \in E_{n} \backslash P$ with $a_{i j}=1$ are either incident to $v_{2}$ or to $v_{4}$. In the remainder of the proof, let $T_{1}:=\left\{i \in V_{n} \mid a_{v_{2}, i}=1\right\}, T_{2}:=\left\{i \in V_{n} \mid a_{w_{i}, i}=1\right\}$ (hence, $v_{1}, v_{3} \in T_{1}$ and $\left.v_{3}, v_{5} \in T_{2}\right)$, and let $T_{0}:=T_{1} \cap T_{2}$. If $i, j \in T_{k}(k=1,2)$, then $a_{i j} \leq-1$.

First, consider the case where $a_{v_{1}, v_{4}} \leq 0$ and also $a_{v_{2}, v_{5}} \leq 0$. If $a_{i j} \leq-2$ for all $i, j \in T_{0}$, then the inequality $a^{T} x \leq 2$ is implied by the sum of two $(S, T)$ inequalities, one with $S=\left\{v_{2}\right\}$ and $T=T_{1}$, and the other with $S=\left\{v_{4}\right\}$ and $T=T_{2}$. If $a_{i j}=-1$ for some $i, j \in T_{0}$, then $a_{v_{2}, v_{4}} \leq-1$, and the inequality $a^{T} x \leq 2$ is implied by an intersection patching of the same two $(S, T)$-inequalities.

If $a_{v_{1}, v_{4}}=1$ or $a_{v_{2}, v_{5}}=1$, then similar arguments show that the inequality $a^{T} x \leq 2$ is either implied by the sum of two $(S, T)$-inequalities, or it is implied 


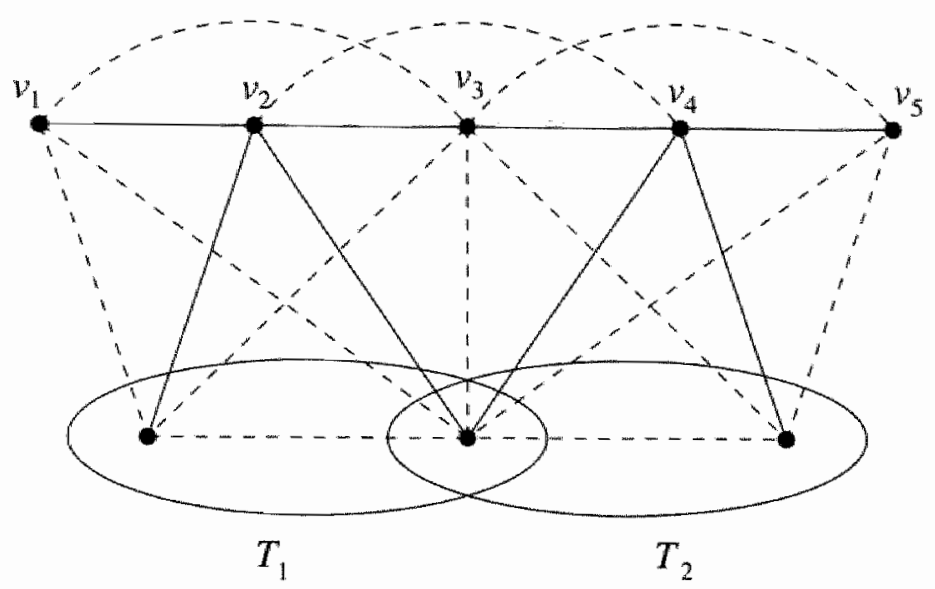

Figure 3.9: Support of an inequality with right-hand side 2.

by an intersection patching of two $(S, T)$-inequalities. This completes the proof of the theorem.

\section{Acknowledgements}

This chapter is based on joint work with Maarten Oosten and Frits Spieksma. 


\section{Chapter 4}

\section{Small polytopes}

\subsection{Introduction}

There are at least three different ways of investigating the structure of a polyhedron. The first one is identifying new classes of facets by using the structure of the underlying combinatorial problem. The second way is lifting, including all kinds of techniques like node cloning, subdivision of edges, graph substitution, and patching. The third possibility is classifying the facets of a small polyhedron by enumerative methods, followed by generalizing the newly obtained facets.

New classes of facets for the clique partitioning polytope were described in Chapter 3, whereas some lifting theorems were described in Chapter 2 and 3. This chapter deals with the third method. In Section 4.2 we give complete descriptions of $P\left(K_{n}\right)$ for $n=4$ and $n=5$, and a partial description for $n=6$. The complete descriptions are obtained with the help of an algorithm implemented by Fukuda [30]. It turns out that all facets of $P\left(K_{4}\right)$ belong to known classes, but $P\left(K_{5}\right)$ contains new facets, which belong to three groups. Generalizations of these new facets make up three new classes of facets of $P\left(K_{n}\right)$ for $n \geq 6$, which are described in Section 4.3 


\subsection{Complete and partial descriptions}

Listing all extreme points of $P\left(K_{n}\right)$ for complete graphs with up to ten vertices is pearuts, but identifying all facets of these polytopes turns out to be very hard, even for $n=6$ we were not able to determine all facets. The clique partitioning polytope associated with $K_{2}$ is simply the interval $[0,1]$ in $\mathbb{R}$, hence $P\left(K_{2}\right)$ has two extreme points which are also facets. The polytope $P\left(K_{3}\right)$ is contained in $\mathbb{R}^{3}$ and has 5 vertices: the origin, all three unit vectors, and the vector $(1,1,1)$. The convex hull of these points has 6 facets. In fact, this polytope is depicted in Figure 1.2 on page 9. The facets of $P\left(K_{3}\right)$ are induced by the nonnegativity constraints and the triangle inequalities. The complete descriptions of $P\left(K_{n}\right)$ for $n=4$ and $n=5$ are given in Table 4.1 and Table 4.2 , respectively. The first column of each table denotes the righthand side of the facet defining inequalities. The second column gives the name of the inequalities (if the corresponding inequality has been given a name in literature). The column labeled "\#" gives the number of different facets in each class. The 2-partition inequalities induced by $S, T \subseteq V_{n}$ are denoted as $(|S|,|T|)$-inequalities.

\begin{tabular}{|c|l|r|}
\hline Rhs & Kind of inequality & $\#$ \\
\hline \hline 0 & Nonnegativity constraints & 6 \\
\hline 1 & Triangle inequalities & 12 \\
& $(1,3)$-inequalities & 4 \\
\hline \hline Total & 22 \\
\hline
\end{tabular}

Table 4.1: Classification of all facets of $P\left(K_{4}\right)$.

From Table 4.1 we abstract that $P\left(K_{4}\right)$ has 22 facets, which belong to three different classes (in fact, the triangle inequalities form a subset of the 2-partition inequalities, and one could say that there are just two classes of facets). All facet defining inequalities for this polytope have their coefficients in $\{0, \pm 1\}$, and even the right-hand side never exceeds 1 . The polytope $P\left(K_{5}\right)$ has 242 facets, which basically form only seven different classes, since all inequalities with right-hand side 1 and the $(2,3)$-inequalities belong to the class of 2 partition inequalities. The complete description of $P\left(K_{5}\right)$ contains three new classes of facets, which make up more than half of the facets of this polytope! "To give some idea of the structure of these inequalities, we present graphical 


\begin{tabular}{|c|l|r|}
\hline Rhs & Kind of inequality & $\#$ \\
\hline \hline 0 & Nonnegativity constraints & 10 \\
\hline 1 & Triangle inequalities & 30 \\
& $(1,3)$-inequalities & 20 \\
& $(1,4)$-inequalities & 5 \\
\hline 2 & $(2,3)$-inequalities & 10 \\
& 2-chorded cycle inequalities & 12 \\
\hline 3 & Claw inequalities $(c=2,|T|=4)$ & 5 \\
& Figure 4.1 (left) & 60 \\
& Figure 4.1 (right) & 60 \\
\hline 5 & Figure 4.2 & 30 \\
\hline \hline Total & 242 \\
\hline
\end{tabular}

Table 4.2: Classification of all facets of $P\left(K_{5}\right)$.

interpretations in Figure 4.1 and Figure 4.2. Solid lines correspond to positive coefficients (default value 1), dotted lines correspond to negative coefficients (default value -1).

Table 4.3 gives a partial description of $P\left(K_{6}\right)$. This polytope has at least 2862 facets, which can be grouped into eleven classes. The new classes of facets found in the complete description of $P\left(K_{5}\right)$ and some simple generalizations of these facets give rise to more than half of the known facets of the clique partitioning polytope of $K_{6}$.

\subsection{New facets}

In this section we describe three new classes of facets for the clique partitioning polytope, which are generalizations of facets found in the complete description of $P\left(K_{5}\right)$. To prove 'facetness' of these generalizations we heavily rely on Theorem 2.13.

The following three theorems each describe a new class of facets of the clique partitioning polytope of a complete graph on 5 or more vertices. 


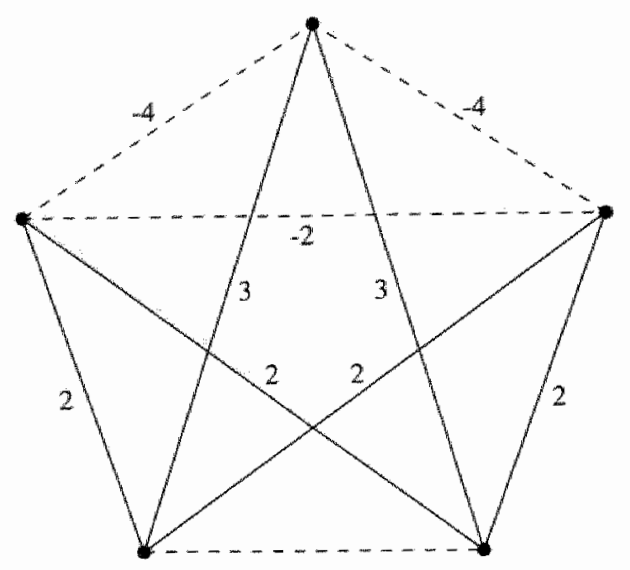

Figure 4.2: Support of facets with right-hand side 5.

of $P\left(K_{5}\right)$. We use Theorem 2.13 to prove that (4.1) defines a facet of $P\left(K_{n}\right)$ for $T_{1}$ and $T_{2}$ of arbitrary size. Define

$$
\begin{aligned}
E^{0}\left(t_{1}, t_{2}\right) & :=E_{n}\left(S \cup\left\{t_{1}, t_{2}\right\}\right) \quad \forall t_{1} \in T_{1}, t_{2} \in T_{2} \\
E^{k}\left(t, t^{\prime}\right) & :=E_{n}\left(\left\{s_{0}, t, t^{\prime}\right\}\right) \quad \forall t, t^{\prime} \in T_{k}(k=1,2)
\end{aligned}
$$

Let $F \subseteq E_{n}$ be any of the edge sets defined by $(4.2)$, then $\operatorname{load}_{\kappa_{n}}(a, F)=$ 3 and $\operatorname{res}_{K_{0}}(a, F)=0$. We already proved that the truncated inequality $\bar{a}_{F}^{T} \bar{x}_{F} \leq \operatorname{load}_{K_{n}}(a, F)=3$ defines a facet of $P\left(V_{n}, F\right)$. For any edge set $F^{\prime}$ defined by (4.3) we have $\operatorname{load}_{K_{n}}\left(a, F^{\prime}\right)=2$ and $\operatorname{res}_{K_{n}}\left(a, F^{\prime}\right)=1$, and $F^{\prime}$ induces a triangle inequality (multiplied by 2 ), which is facet defining. The intersection graph of all edge sets (4.2) and (4.3) with respect to $a^{T} x \leq a_{0}$ is comnected, and the union of all these sets is $E_{n}\left(S \cup T_{1} \cup T_{2}\right)$. This means that all conditions of Theorem 2.13 are satisfied, and hence (4.1) defines a facet of $P\left(V_{n}, E_{n}\left(S \cup T_{1} \cup T_{2}\right)\right)$. Corollary 2.8 implies that (4.1) also defines a facet of $P\left(K_{n}\right)$.

Theorem 4.2 Let $K_{n}=\left(V_{n}, E_{n}\right)$ be a complete graph on at least 5 vertices, and let $S=\left\{s_{0}, s_{1}\right\}, T \subseteq V_{n}$ be two nonempty disjoint sets, $|T| \geq 3, t_{1}, t_{2} \in T$. The inequality (see also Figure 4.1 (left) and Figure 4.3 (left))

$$
\begin{aligned}
& x\left(E_{n}\left(\left\{s_{0}, t_{1}, t_{2}\right\}\right)+2 \cdot x\left(E_{n}\left(\left\{s_{1}\right\}, T\right)\right)\right. \\
& -2 \cdot x\left(E_{n}(S)\right)-2 \cdot x\left(E_{n}(T)\right) \leq 3
\end{aligned}
$$



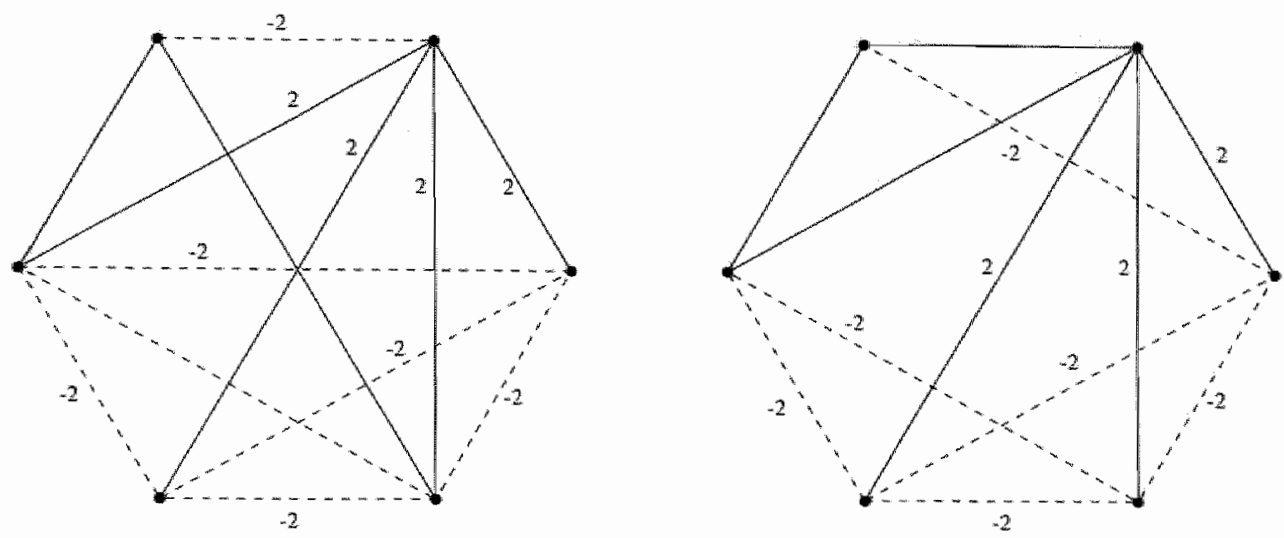

Figure 4.3: Support of facets with right-hand side 3.

is valid and facet defining for $P\left(K_{n}\right)$.

Proof Validity: Denote (4.4) by $a^{T} x \leq a_{0}$. All edges $\{i, j\} \in E_{n}$ with $a_{i j}>0$ are either incident to $s_{0}$ or to $s_{1}$. Let $A \subseteq E_{n}$ be a clique partition of $K_{n}$. We distinguish two situations.

(i) $\left|A \cap E_{n}\left(\left\{s_{0}, t_{1}, t_{2}\right\}\right)\right| \in\{0,1\}$. Then $A$ satisfies the following inequalities, implying (4.4).

$$
\begin{aligned}
2 \cdot x\left(E_{n}\left(\left\{s_{1}\right\}, T\right)\right)-2 \cdot x\left(E_{n}(T)\right) & \leq 2 \\
x\left(E_{n}\left(\left\{s_{0}, t_{1}, t_{2}\right\}\right)\right. & \leq 1
\end{aligned}
$$

(ii) $\left|A \cap E_{n}\left(\left\{s_{0}, t_{1}, t_{2}\right\}\right)\right|=3$. If $\left\{s_{0}, s_{1}\right\} \in A$, then (4.4) is implied by (4.5) and the inequality $x\left(E_{n}\left(\left\{s_{0}, t_{1}, t_{2}\right\}\right)-2 x(E(S)) \leq 1\right.$. If $\left\{s_{0}, s_{1}\right\} \notin A$, then $(4.4)$ is implied by

$$
\begin{aligned}
2 \cdot x\left(E_{n}\left(\left\{s_{1}\right\}, T \backslash\left\{t_{1}, t_{2}\right\}\right)\right)-2 \cdot x\left(E_{n}\left(T \backslash\left\{t_{1}, t_{2}\right\}\right)\right) & \leq 2 \\
x_{s_{0}, t_{1}}+x_{s_{0}, t_{3}}-x_{t_{1}, t_{2}} & \leq 1
\end{aligned}
$$

Facet defining: If $|T|=3$ the support of (4.4) has 5 vertices. It follows from inspection that 10 out of the 52 clique partitions of $K_{5}$ satisfy (4.4) at 

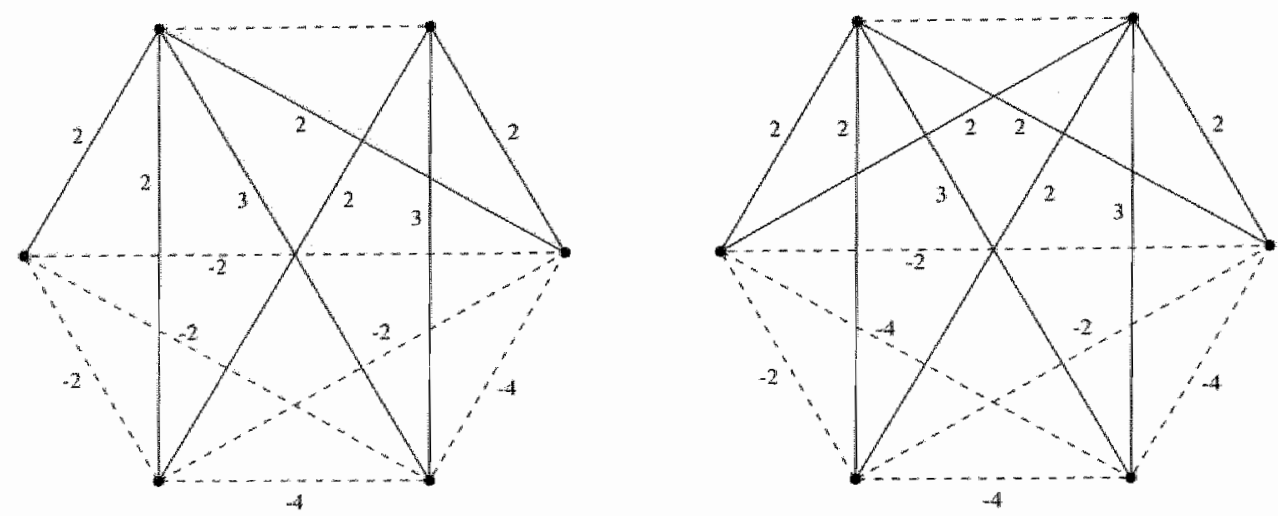

Figure 4.4: Support of facets with right-hand side 5.

equality. Moreover, the incidence vectors of these ten clique partitions are affinely independent. Hence, for $|T|=3$ inequality (4.4) defines a facet of $P\left(K_{5}\right)$. We use Theorem 2.13 to prove that (4.4) defines a facet of $P\left(K_{n}\right)$ for $|T| \geq 4$. Define

$$
\begin{aligned}
E^{0}(t) & :=E_{n}\left(S \cup\left\{t_{1}, t_{2}, t\right\}\right) \quad \forall t \in T \backslash\left\{t_{1}, t_{2}\right\} \\
E^{\prime}\left(t, t^{\prime}\right) & :=E_{n}\left(\left\{s_{1}, t, t^{\prime}\right\}\right) \quad \forall t, t^{\prime} \in T \backslash\left\{t_{1}, t_{2}\right\}
\end{aligned}
$$

Then $\operatorname{load}_{K_{n}}\left(a, E^{0}(t)\right)=3$ and $\operatorname{res}_{K_{n}}\left(a, E^{0}(t)\right)=0$ for all $t \in T \backslash\left\{t_{1}, t_{2}\right\}$, and load $K_{n}\left(a, E^{\prime}\left(t, t^{\prime}\right)\right)=2$ and $\operatorname{res}_{K_{n}}\left(a, E^{\prime}\left(t, t^{\prime}\right)\right)=1$ for all $t, t^{\prime} \in T \backslash\left\{t_{1}, t_{2}\right\}$. Notice that for any edge set $F \subseteq E_{n}$ defined by (4.7) or (4.8) the truncated incquality $a_{F}^{T} x_{F} \leq \operatorname{load}_{K_{n}}(a, F)$ defines a facet of $P\left(V_{n}, F\right)$. The intersection graph of the edge sets (4.7) and (4.8) with respect to $a^{T} x \leq a_{0}$ is connected, and the edge sets cover all edges in $E_{n}(S \cup T)$. It follows from Theorem 2.13 that (4.4) defines a facet of $P\left(V_{n}, E_{n}(S \cup T)\right)$, and Corollary 2.8 shows that is also defines a facet of $P\left(K_{n}\right)$.

Theorem 4.3 Let $K_{n}=\left(V_{n}, E_{n}\right)$ be a complete graph on at least 5 vertices, and let $S=\left\{s_{0}, s_{1}, s_{2}\right\}, T_{0}, T_{1}, T_{2} \subseteq V_{n}$ be four disjoint sets, $\left|T_{0}\right| \geq 2$ (the sets 
$T_{1}$ and $T_{2}$ may be empty). The inequality (see also Figure 4.2 and Figure 4.4)

$3 \cdot x\left(E_{n}\left(\left\{s_{0}\right\},\left\{s_{1}, s_{2}\right\}\right)\right)-x_{s_{1}, s_{2}}+2 \cdot x\left(E_{n}\left(\left\{s_{1}\right\}, T_{0} \cup T_{1}\right)\right)$

$+2 \cdot x\left(E_{n}\left(\left\{s_{2}\right\}, T_{0} \cup T_{2}\right)\right)-4 \cdot x\left(E_{n}\left(\left\{s_{0}\right\}, T_{0}\right)\right)$

$-2 \cdot x\left(E_{n}\left(\left\{s_{0}\right\}, T_{1} \cup T_{2}\right)\right)-2 \cdot x\left(E_{n}\left(T_{0} \cup T_{1}\right) \cup E_{n}\left(T_{0} \cup T_{2}\right)\right) \leq 5$

is valid and facet defining for $P\left(K_{n}\right)$.

Proof Validity: First, notice that the inequality

$$
\begin{aligned}
& x\left(E_{n}\left(\left\{s_{1}\right\}, T_{0} \cup T_{1}\right)\right)+x\left(E_{n}\left(\left\{s_{2}\right\}, T_{0} \cup T_{2}\right)\right) \\
& -x_{s_{1}, s_{2}}-x\left(E_{n}\left(T_{0} \cup T_{1}\right) \cup E_{n}\left(T_{0} \cup T_{2}\right)\right) \leq 2
\end{aligned}
$$

is valid for $P\left(K_{n}\right)$ (it is even facet defining, since it is a generalized 2-chorded path inequality, see (3.24)). Let $A \subseteq E_{n}$ be a clique partition. If one distinguishes the situations $\left|A \cap E_{n}\left(\left\{s_{0}\right\},\left\{s_{1}, s_{2}\right\}\right)\right|=k$ for $k=0,1,2$, using inequality (4.10), it is easy to see that $(4.9)$ is valid for $P\left(K_{n}\right)$.

Facet defining: Denote (4.9) by $a^{T} x \leq a_{0}$. If $T_{1}=T_{2}=\varnothing$ and $\left|T_{0}\right|=2$ the support of (4.1) has 5 vertices. It follows from inspection that 10 out of the 52 clique partitions of $K_{5}$ satisfy (4.9) at equality. Moreover, the incidence vectors of these ten clique partitions are affinely independent. Hence, for $T_{1}=T_{2}=\varnothing$ and $\left|T_{0}\right|=2$ inequality (4.9) defines a facet of $P\left(K_{5}\right)$. We use Theorem 2.13 to prove that (4.9) defines a facet of $P\left(K_{n}\right)$ for arbitrary nonempty sets $T_{0}$, $T_{1}$ and $T_{2}$. If $\left|T_{0}\right| \geq 3$ define

$$
E^{0}\left(t, t^{\prime}\right):=E_{n}\left(S \cup\left\{t, t^{\prime}\right\}\right) \quad \forall t, t^{\prime} \in T_{0}
$$

If $\left|T_{k}\right|=1(k=1,2)$ define

$$
E^{k}\left(s_{k}, t_{k}, t_{0}\right):=E_{n}\left(\left\{s_{k}, t_{k}, t_{0}\right\}\right) \quad \forall t_{0} \in T_{0,}, t_{k} \in T_{k}
$$

If $\left|T_{k}\right| \geq 2(k=1,2)$ define

$$
E^{k}\left(s_{k}, t_{k}, t_{k}^{\prime}, t_{0}\right):=E_{n}\left(\left\{s_{k}, t_{k}, t_{k}^{\prime}, t_{0}\right\}\right) \quad \forall t_{0} \in T_{0}, t_{k}, t_{k}^{\prime} \in T_{k}
$$


The load of the edge sets (4.11) with respect to (4.9) equals 5, and hence the residual equals 0 . The load of the edge sets (4.12) and (4.13) equals 2 , and the residual equals 3. Furthermore, for any edge set $F \subseteq E_{n}$ defined by (4.11)-(4.13) the corresponding truncation of $a^{T} x \leq a_{0}$ defines a facet of $P\left(V_{n}, F\right)$, and the intersection graph of all these edge sets with respect to $a^{T} x \leq a_{0}$ is comnected. Theorem 2.13 shows that (4.9) defines a facet for the clique partitioning polytope corresponding to the graph $G=\left(V_{n}, F\right)$, where $F$ is the union of the edge sets considered in (4.11)-(4.13). Now, let $\left\{e_{1}, \ldots, e_{k}\right\}=E_{n} \backslash F$. To prove that (4.9) also defines a facet of $P\left(K_{n}\right)$ it suffices to identify, for each edge $e_{i} \in E_{n} \backslash F$, a clique partition of $K_{n}$ contaiming $e_{i}$, but not containing any of the edges $e_{i+1}, \ldots, e_{k}$. We start with the edges in $E_{n}\left(\left\{s_{0}\right\}, T_{1}\right)$. Take $t_{0} \in T_{0}$ and $t_{1} \in T_{1}$, and let $A:=$ $E_{n}\left(\left\{s_{0}, s_{\Perp}, t_{1}\right\}\right) \cup\left\{\left\{s_{2}, t_{0}\right\}\right\}$. Obviously, $a^{T} \chi^{A}=a_{0}$. Because of symmetry we can do the same for the edges in $E_{n}\left(\left\{s_{0}\right\}, T_{2}\right)$. Next, for all $t \in T_{1} \cup T_{2}$ we take $A:=E_{n}(S \cup\{t\})$, and again $a^{T} \chi^{A}=a_{0}$. Finally, for $\left\{t_{1}, t_{2}\right\} \in E_{n}\left(T_{1}, T_{2}\right)$ we take for $A$ the union of the edges $\left\{s_{0}, s_{1}\right\},\left\{t_{1}, t_{2}\right\}$, and $\left\{s_{2}, t_{0}\right\}$ for some $t_{0} \in T_{0}$. This proves that (4.9) defines a facet of the clique partitioning polytope of the complete graph with vertex set $S \cup T_{0} \cup T_{1} \cup T_{2}$, and Corollary 2.8 shows that it also defines a facet of $P\left(K_{n}\right)$. 


\section{Chapter 5}

\section{Strength}

\subsection{Introduction}

Many combinatorial optimization problems can be formulated as an integer linear program, such that the polyhedron $P$ corresponding to the underlying combinatorial problem is exactly the convex hull of all feasible points to the integer program. When it comes to solving such combinatorial problems one often uses a linear programming package in combination with a branchand-cut algorithm. The success of this approach depends on several issues. Solving the linear programming relaxation usually results in fractional instead of integer optimal solutions, and in order to arrive at an integral solution one has to resort to branching, or search for inequalities that are satisfied by all integral solutions, but not by the current fractional solution. Since branching is very time consuming in general, searching violatied inequalities is mostly preferred. It seems natural to search for violated facet defining inequalities, since these inequalities are in some sense the strongest valid inequalities for $P$, and there is at least one facet defining inequality that is violated by the current fractional solution. This cutting plane approach immediately gives rise to the following question. How hard is it to find violated facet defining inequalities, i.e. what is the complexity of separation for a class of facets? The answer to this question, at least for some classes of facets of the clique partitioning polytope, is postponed to the next chapter. Before puting a lot of effort in solving the separation problem associated with a class of facets it would be desirable to have some indication of the usefulness of these facets 
in a cutting plane algorithm. Therefore, in the next section we present three different measures for the "strength" of an inequality with respect to a given polyhedron. In the last two sections of the chapter we illustrate the concept of strength using the clique partitioning polytope.

\subsection{Measures for strength}

When $Q \subseteq \mathbb{R}^{n}$ is a polyhedron originating from a combinatorial optimization problem, it is not very likely that one has a complete linear description of $Q$, but instead only a partial description which gives rise to a polyhedron $P \subseteq \mathbb{R}^{n}$ which is a relaxation of $Q$. If $Q$ is contained in the halfspace $H:=\{x \in$ $\left.\mathbb{R}^{n} \mid a^{T} x \leq a_{0}\right\}$ and $P$ is not, the strength of the valid inequality $a^{T} x \leq a_{0}$ with respect to $Q$ can be defined as the maximal (Euclidean) distance between the boundary of $H$ and a point in the polyhedron $\left\{x \in \mathbb{R}^{n} \mid a^{T} x \geq a_{0}\right\}$. If $P \subseteq H$ the strength can be defined as 0 .

Another measure for strength is based on volumes. Consider a polyhedron $P \subseteq \mathbb{R}^{n}$ that is a relaxation of a full dimensional polyhedron $Q \subseteq \mathbb{R}^{n}$, and let $a^{T} x \leq a_{0}$ be a valid inequality for $Q$. Since $Q$ is full dimensional, so is $P$, and the strength of $a^{T} x \leq a_{0}$ with respect to $P$ can be defined as the volume of the polyhedron $\left\{x \in P \mid a^{T} x \geq a_{0}\right\}$, i.e. the volume of $P$ cut off by the hyperplane $a^{T} x=a_{0}$. If $a^{T} x \leq a_{0}$ defines a facet of $Q$, then we can also define the strength of this inequality with respect to $Q$. Clearly, the polyhedron $Q$ can be written as $Q=\left\{x \in \mathbb{R}^{n} \mid A x \leq b\right\}$ for some real $m \times n$ matrix $A$ and some vector $b \in \mathbb{R}^{m}$, such that there is a one-to-one relation between the rows of $A$ and the facets of $Q$. Let $a_{k} \in \mathbb{R}^{n}$ denote the $k$-th row of $A$, and let $A^{\prime}$ and $b^{\prime}$ be obtained by deleting the $k$-th row of $A$ and $b$, respectively. The strength of $a_{k}^{T} x \leq b_{k}$ with respect to $Q$ can be defined as the volume of the polyhedron $\left\{x \in \mathbb{R}^{n} \mid A^{\prime} x \leq b^{\prime}, a_{k}^{T} x \geq b_{k}\right\}$. In words, the strength of the facet defining inequality $a^{T} x \leq a_{0}$ with respect to $Q$ is the increase in volume if this inequality would be ommitted in the linear description of $Q$. Notice that both definitions result in a nonnegative strength, and that the strength can be infinite. The strength according to the first definition depends on the relaxation $P$ of $Q$, which only makes sense if $P$ is obtained from $Q$ in a natural way, for instance as a linear programming or Lagrangian relaxation. Both measures for strength suffer from the fact that calculating the volume of an n-dimensional polyhedron is extremely difficult, see Lee and Morris [50] 
for a geometric comparison of combinatorial polytopes. Moreover, volumes are positive only for full dimensional polyhedra, whereas many combinatorial problems give rise to polyhedra that are not full dimensional.

A third measure for strength requires a probability distribution. Let $V_{Q}$ be the set of extreme points (vertices) of a polyhedron $Q \subseteq \mathbb{R}^{n}$, and let $\varphi: V_{Q} \rightarrow[0,1]$ be a probability distribution on the vertices of $Q$, i.e. $\varphi(x) \geq 0$ for all $x \in V_{Q}$, and $\sum_{x \in V_{Q}} \varphi(x)=1$. The strength of a valid inequality $a^{T} x \leq a_{0}$ with respect to $Q$ can be defined as

$$
\sum_{\substack{x \in V_{Q} \\ a^{T} x=a_{0}}} \varphi(x)
$$

In words, the strength of the inequality $a^{T} x \leq a_{0}$ with respect to $Q$ is the total probability mass on the extreme points of the face $\left\{x \in Q \mid a^{T} x=a_{0}\right\}$. Depending on the combinatorial problem from which $Q$ originates there might be a natural probability distribution. The probability distribution over the vertices of $Q$ can allso be given implicitly by another probability distribution of the objective vector $c \in \mathbb{R}^{n}$. For instance, all coefficients $c_{1}, \ldots, c_{n} \in \mathbb{R}$ can be taken from a uniform distribution on the interval $[-1,1]$, and for all $x \in V_{Q}$ we take $\varphi(x)$ equal to the probability that $x$ is an optimal solution to $\max \left\{c^{T} x \mid x \in Q\right\}$. Another possibility is taking $c \in \mathbb{R}^{n}$ from a uniform distribution on the $n$-dimensional unit sphere, so that the 'direction' $c$ points to is uniformly distributed in $\mathbb{R}^{n}$. This distribution can be achieved by taking all coefficients $c_{1}, \ldots, c_{n} \in \mathbb{R}$ from a normal distribution with mean 0 and variance $\sigma^{2}>0$.

In the subsections below we give three precise definitions for the strength of an inequality with respect to a given polyhedron, which are based on the ideas described above. In Section 5.3 and 5.4 we discuss the strength of facets of the clique partitioning polytope according to these measures.

\subsubsection{Counting extreme points}

Let $a^{T} x \leq a_{0}$ be an inequality, and let $P \subseteq \mathbb{R}^{n}$ be a polytope that is contained in the halfspace $\left\{x \in \mathbb{R}^{n} \mid a^{T} x \leq a_{n}\right\}$. If all feasible solutions have the same probability of being optimal, one could say that the more extreme points $x \in P$ 
satisfy $a^{T} x=a_{0}$ the stronger the inequality is. This observation is the basis for our first measure for the strength of an inequality with respect to a given polytope.

Definition 5.1 Let $P \subseteq \mathbb{R}^{n}$ be a polytope contained in the halfspace $\{x \in$ $\mathbb{R}^{n}\left\{a^{T} x \leq a_{0}\right\}$, and let $V_{P}$ be the set of extreme points (vertices) of $P$. The strength $S_{1}(a, P)$ of $a^{T} x \leq a_{0}$ with respect to $P$ is defined as

$$
S_{1}(a, P):=\frac{\left|\left\{x \in V_{P}: a^{T} x=a_{0}\right\}\right|}{\left|V_{P}\right|}
$$

Notice that $0 \leq S_{1}(a, P) \leq 1$ for any valid inequality $a^{T} x \leq a_{0}$ for $P$, and that the strength is 0 if and only if $P \subseteq\left\{x \in \mathbb{R}^{n} \mid a^{T} x<a_{0}\right\}$. For any inequality $a^{T} x \leq a_{0}$ valid for $P$ there is a facet defining inequality $b^{T} x \leq b_{0}$ for $P$ with $S_{1}(b, P) \geq S_{1}(a, P)$, because any proper face of $P$ is contained in a facet. This measure for the strength of an inequality occurs as a special case of the last example given above, namely if $\varphi: V_{P} \rightarrow[0,1]$ is defined by $\varphi(x)=\frac{1}{\left|V_{P}\right|}$ for all $x \in V_{P}$. The idea to count extreme points of facets is also used in Naddef and Rinaldi [57] for the symmetric traveling salesman polytope.

\subsubsection{The ratio measure}

Let $Q \subseteq \mathbb{R}^{n}$ be a polytope contained in the halfspace $\left\{x \in \mathbb{R}^{n} \mid a^{T} x \leq a_{0}\right\}$, and let $P$ be a relaxation of $Q$ that is not contained in this halfspace.

When maximizing $a^{T} x$ over $P$ we will find an optimal value $z>a_{0}$. The larger $z$ is, the more profit we will have from adding the valid constraint $a^{T} x \leq a_{0}$ to the relaxation $P$ of $Q$. The maximum $z:=\left\{a^{T} x \mid x \in P\right\}$ will be more than $a_{0}$, and the larger the value of $z$, the more profit we will have from adding the valid constraint $a^{T} x \leq a_{0}$ to the relaxation $P$ of $Q$. This observation leads to the second measure for the strength of an inequality with respect to some polytope.

Definition 5.2 Let $H=\left\{x \in \mathbb{R}^{n_{1}} \mid a^{T} x \leq a_{0}\right\}$ be a halfspace in $\mathbb{R}^{n}$ containing the origin as an interior point (i.e. $a_{0}>0$ ), let $Q \subseteq H$ be a polyhedron, and 
let $P \supseteq Q$ be a relaxation of $Q$ not contained in the halfspace $H$. The strength $S_{2}(a, P)$ of $a^{T} x \leq a_{0}$ with respect to $P$ is defined as

$$
S_{2}(a, P):=\frac{\max \left\{a^{T} x \mid x \in P\right\}}{a_{0}}
$$

The conditions of the definition ensure that $S_{2}(a, P) \geq 1$. If $a^{T} x$ is unbounded over $P$ then the inequality $a^{T} x \leq a_{0}$ has infinite strength. If $a^{T} x \leq a_{0}$ defines a nonempty face of $Q$ (i.e. $\left\{x \in Q \mid a^{T} x=a_{0}\right\} \neq \varnothing$ ), this measure denotes the ratio of the optimal value when maximizing $a^{T} x$ over $P$ and $Q$, respectively. If $Q \subseteq H_{\varepsilon}:=\left\{x \in \mathbb{R}^{n} \mid a^{T} x \leq a_{0}-\varepsilon\right\}$ for some $0<\varepsilon<a_{0}$, then the inequality defining $H_{E}$ is stronger (with respect to $P$ ) than the inequality defining $H$, which makes sense, because the hyperplane $a^{T} x \leq a_{0}-\varepsilon$ cuts off a larger part of $P$ than $a^{T} x \leq a_{0}$ does.

Goemans [33] considered a similar measure for the strength of an inequality with respect to a polyhedron $P \subseteq \mathbb{R}_{+}^{n}$ that is a relaxation of a polyhedron $Q \subseteq \mathbb{R}_{+}^{n}$ of blocking type, i.e. $x \in Q$ and $y \geq x$ imply $y \in Q$. Given $\alpha \geq 1$, $P$ is an $\alpha$-relaxation of $Q$ (or $Q$ is an $\alpha$-strengthening of $P$ ) if $Q \supseteq \alpha P=$ $\{\alpha \cdot x \mid x \in P\}$. Any $\alpha$-relaxation is also a $\beta$-relaxation for $\beta \geq \alpha$. Next, let $t(P, Q)$ denote the minimum value of $\alpha$ such that $P$ is an $\alpha$-relaxation of $Q$, that is

$$
t(P, Q):=\min \{\alpha \geq 1 \mid Q \supseteq \alpha P\}
$$

Notice that $t(P, Q) \geq 1, t(P, Q)=1$ if and only if $P=Q$, and that $t(P, Q)$ can be infinite. Given $a \in \mathbb{R}_{+}^{n}$ and $a_{0} \geq 0$, Goemans defines the strength of the inequality $a^{T} x \geq a_{0}$ with respect to $P$ as

$$
\frac{a_{0}}{\min \left\{a^{T} x \mid x \in P\right\}}
$$

He proves that, under the conditions mentioned above, $t(P, Q)$ is equal to the maximum strength with respect to $P$ of a facet defining inequality for $Q$. The ratio measure is also considered by Oosten [59] for zero-identical polyhedra. Two polyhedra $P, Q \subseteq \mathbb{R}^{n}$ are called zero-identical if and only if $P \subseteq\left\{x \mid a^{T} x \leq 0\right\}$ is equivalent to $Q \subseteq\left\{x \mid a^{T} x \leq 0\right\}$ for all $a \in \mathbb{R}^{n}$. 


\subsubsection{The distance measure}

The third measure for the strength of an inequality is based on the Euclidean distance in $\mathbb{R}^{n}$ between a hyperplane and a face of a polyhedron. A more precise formulation is given in the following definition.

Definition 5.3 Let $H=\left\{x \in \mathbb{R}^{n} \mid a^{T} x \leq a_{0}\right\}$ be a halfspace in $\mathbb{R}^{n}$, let $Q \subseteq H$ be a polyhedron, and let $P \supseteq Q$ be a relaxation of $Q$ not contained in the halfspace $H$. The strength $S_{3}(a, P)$ of $a^{T} x \leq a_{0}$ with respect to $P$ is defined as

$$
S_{3}(a, P):=\frac{\max \left\{a^{T} x \mid x \in P\right\}-a_{0}}{\|a\|}
$$

In words, the strength of $a^{T} x \leq a_{0}$ with respect to $P$ is the Euclidean distance between the hyperplane $a^{T} x=a_{0}$ and the optimal face of $P$ when maximizing $a^{T} x$.

All three measures for the strength of an inequality are invariant under scaling of the vector $\left(a, a_{0}\right) \in \mathbb{R}^{n+1}$, and measure $S_{1}(a, P)$ and $S_{3}(a, P)$ are also invariant under simultaneous translations of $P$ and the hyperplane $a^{T} x=a_{0}$.

In the next section we calculate the strength, according to all three measures, of all known facets for some small clique partitioning polytopes.

\subsection{The strength of small facets}

Two out of the three definitions of strength given in the previous section are defined with respect to some relaxation of a polyhedron. For the clique partitioning polytope $P\left(K_{n}\right)$ we defined the strength of a valid inequality with respect to the polytope $P_{L P}\left(K_{n}\right)$, which is the set of feasible points of the linear programming relaxation of (2.1) for the complete graph $K_{n}$. The strength of the facets of $P\left(K_{n}\right)$ for $n=4,5,6$ is listed in Tables 5.1, 5.2, and 5.3. These tables are organized as follows. The first column gives the right-hand side of a facet, the second column gives the name of the facet defining inequality or we refer to a figure showing the support of the inequality. The column labeled 


\begin{tabular}{|c|l|r|r|r|r|r|}
\hline Rhs & Kind of inequality & $\#$ & $S_{1}$ & \multicolumn{1}{c|}{$S_{2}$} & \multicolumn{1}{c|}{$S_{3}$} \\
\hline 0 & Nonneg. constraints & 6 & 10 & - & - & - \\
\hline 1 & Triangle inequalities & 12 & 8 & - & - & - \\
& $(1,3)$-inequalities & 4 & 6 & $3 / 2$ & $1 /(2 \sqrt{6})$ & 0.2041 \\
\hline \hline
\end{tabular}

Table 5.1: The strength of facets of $P\left(K_{4}\right)$.

"\#" gives the number of facets in this class. The last three columns, labeled " $S_{1}$ ", " $S_{2}$ ", and " $S_{3}$ " present the different measures for strength. The column " $S_{1}$ " gives the number of extreme points of $P\left(K_{n}\right)$ that belong to the facet induced by the particular inequality. At the bottom of this column we give the total number of vertices of $P\left(K_{n}\right)$, so it can be seen that $P\left(K_{4}\right)$ has 15 vertices and $P\left(K_{5}\right)$ has 52 vertices. Column " $S_{2}$ " speaks for itself, and column " $S_{3}$ " is split into two parts. The first part gives the exact strength according to Definition 5.3, and the second part contains a decimal representation of the strength.

Notice that the ranking according to the strength of the classes of facets depends on the measure used. For instance, the facets for $P\left(K_{6}\right)$ shown in Figure 4.4 (right) have only 20 extreme points, but according to Definition 5.3 it is the strongest class of facets but one.

\subsection{The strength of facets of $P\left(K_{n}\right)$}

The strength of an inequality, according to one of the definitions given in Section 5.2, with respect to the clique partitioning polytope or its linear programming relaxation can easily be computed for fixed $n \in \mathbb{N}$, either by counting extreme points or by solving a linear programming problem. This approach is not suited for computing the strength of an inequality with respect to $P\left(K_{n}\right)$ for arbitrary $n \in \mathbb{N}$. Nevertheless, it is possible to find a closed formula for the strength of an inequality when considering complete graphs of arbitrary size. Let us first count the number of vertices of $P\left(K_{n}\right)$. It is well known that the number of partitions of a set of $n \geq 1$ objects into $k \geq 1$ nonempty disjoint subsets is equal to $S(n, k)$, the Stirling number of the second kind, so that the 


\begin{tabular}{|c|l|r|r|r|r|r|}
\hline Rhs & Kind of inequality & $\#$ & $S_{1}$ & \multicolumn{1}{|c|}{$S_{2}$} & \multicolumn{2}{c|}{$S_{3}$} \\
\hline \hline 0 & Nonneg. constraints & 10 & 37 & - & - & - \\
\hline 1 & Triangle inequalities & 30 & 26 & - & - & - \\
& $(1,3)$-inequalities & 20 & 21 & $3 / 2$ & $1 /(2 \sqrt{6})$ & 0.2041 \\
& $(1,4)$-inequalities & 5 & 10 & 2 & $1 / \sqrt{10}$ & 0.3162 \\
\hline 2 & $(2,3)$-inequalities & 10 & 17 & $3 / 2$ & $1 / \sqrt{10}$ & 0.3162 \\
& 2 -chorded cycles & 12 & 10 & $5 / 4$ & $1 /(2 \sqrt{10})$ & 0.1581 \\
\hline 3 & Claw inequalities & 5 & 10 & $4 / 3$ & $1 / \sqrt{22}$ & 0.2132 \\
& Figure 4.1 (left) & 60 & 10 & $4 / 3$ & $1 /(3 \sqrt{3})$ & 0.1925 \\
& Figure 4.1 (right) & 60 & 10 & $4 / 3$ & $1 / \sqrt{23}$ & 0.2085 \\
\hline 5 & Figure 4.2 & 30 & 10 & $7 / 5$ & $2 / \sqrt{71}$ & 0.2374 \\
\hline \hline Total & & 242 & 52 & & & \\
\hline
\end{tabular}

Table 5.2: The strength of facets of $P\left(K_{5}\right)$.

number $B_{n} \in \mathbb{N}$ of clique partitions of $K_{n}$ is given by

$$
B_{n}:=\sum_{k=1}^{n} S(n, k)
$$

A closed formula for $S(n, k)$ reads

$$
S(n, k):=\frac{1}{k !} \sum_{l=0}^{k}(-1)^{k-l}\left(\begin{array}{l}
k \\
l
\end{array}\right) l^{n}
$$

see e.g. Van Lint and Wilson [51]. The numbers $B_{n}$ are known as the Bell numbers, and it can be shown that

$$
B_{n}:=\frac{1}{e} \sum_{k=0}^{\infty} \frac{k^{n}}{k !}
$$

The following theorem shows that the strength, according to Definition 5.1, of the nonnegativity constraints with respect to $P\left(K_{n}\right)$ tends to 1 if $n \rightarrow \infty$. 


\begin{tabular}{|c|c|c|c|c|c|c|}
\hline Rhs & Kind of inequality & $\#$ & $S_{1}$ & $S_{2}$ & \multicolumn{2}{|c|}{$S_{3}$} \\
\hline 0 & Nonneg. constraints & 15 & 151 & - & - & - \\
\hline \multirow[t]{4}{*}{1} & Triangle inequalities & 60 & 90 & - & - & 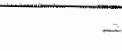 \\
\hline & $(1,3)$-inequalities & 60 & 81 & $3 / 2$ & $\mathbb{1} /(2 \sqrt{6})$ & 0.2041 \\
\hline & $(1,4)$-inequalities & 30 & 44 & 2 & $1 / \sqrt{10}$ & 0.3162 \\
\hline & $(1,5)$-inequalities & 6 & 15 & $5 / 2$ & $3 /(2 \sqrt{15})$ & 0.3873 \\
\hline \multirow[t]{7}{*}{2} & $(2,3)$-inequalities & 60 & 66 & $3 / 2$ & $1 / \sqrt{10}$ & 0.3162 \\
\hline & $(2,4)$-inequalities & 15 & 52 & 2 & $2 / \sqrt{15}$ & 0.5164 \\
\hline & 2-chorded cycles & 72 & 35 & $5 / 4$ & $1 /(2 \sqrt{10})$ & 0.1581 \\
\hline & 2-chorded paths & 180 & 29 & $3 / 2$ & $1 /(2 \sqrt{3})$ & 0.2887 \\
\hline & Comp. 2-part. (I) & 360 & 27 & $4 / 3$ & $2 /(3 \sqrt{10})$ & 0.2108 \\
\hline & Comp. 2-part. (II) & 180 & 33 & $3 / 2$ & $1 / \sqrt{11}$ & 0.3015 \\
\hline & Wheel inequalities & 72 & 25 & $5 / 4$ & $1 /(2 \sqrt{10})$ & 0.1581 \\
\hline \multirow[t]{7}{*}{3} & Claw ineqs. $(|T|=4)$ & 30 & 36 & $4 / 3$ & $1 / \sqrt{22}$ & $0 . \overline{2132}$ \\
\hline & Claw ineqs. $(|T|=5)$ & 6 & 20 & $5 / 3$ & $2 / \sqrt{30}$ & 0.3651 \\
\hline & Lifted claw ineqs. & 30 & 15 & $\mathbb{1 1} / 6$ & $5 /(2 \sqrt{47})$ & 0.3647 \\
\hline & Figure 4.1 (left) & 360 & 35 & $4 / 3$ & $1 /(3 \sqrt{3})$ & 0.1925 \\
\hline & Figure 4.1 (right) & 360 & 35 & $4 / 3$ & $1 / \sqrt{23}$ & 0.2085 \\
\hline & Figure 4.3 (left) & 180 & 19 & $5 / 3$ & $2 / \sqrt{43}$ & 0.3050 \\
\hline & Figure 4.3( & 180 & 19 & $5 / 3$ & $2 / \sqrt{39}$ & 0.3203 \\
\hline \multirow[t]{3}{*}{5} & Figure 4.2 & 180 & 35 & $7 / 5$ & $2 / \sqrt{71}$ & 0.2374 \\
\hline & Figure 4.4( & 360 & 17 & $8 / 5$ & $3 / \sqrt{87}$ & 0.3216 \\
\hline & Figure 4.4 (right) & 60 & 20 & $9 / 5$ & $4 / \sqrt{103}$ & 0.3941 \\
\hline 6 & Claw ineqs. $(|T|=5)$ & 6 & 15 & $5 / 4$ & $3 /(2 \sqrt{55})$ & 0.2022 \\
\hline \multicolumn{2}{|l|}{10} & 362 & 203 & & & \\
\hline
\end{tabular}

Table 5.3: The strength of facets of $P\left(K_{6}\right)$. 
Theorem 5.4 Let $K_{n}=\left(V_{n}, E_{n}\right)$ be the complete graph on $n$ vertices, let $i, j \in V_{n}$ be two distinct vertices, and let $-x_{i j}=a^{T} x \leq a_{0}=0$ denote the nonnegativity constraint corresponding to edge $\{i, j\} \in E_{n}$. Then

$$
\lim _{n \rightarrow \infty} S_{1}\left(a, P\left(K_{n}\right)\right)=1
$$

Proof The number of clique partitions of $K_{n}$ is given by (5.1). All clique partitions $x \in P\left(K_{n}\right)$ satisfying $x_{i j}=1$ have the vertices $i$ and $j$ in the same clique. Contracting edge $\{i, j\}$ (or equivalently, considering $i$ and $j$ as one vertex) gives that the number of clique partitions $x \in P\left(K_{n}\right)$ with $x_{i j}=1$ is equal to $B_{n-1}$. Since

$$
\lim _{n \rightarrow \infty} \frac{B_{n-1}}{B_{n}}=0
$$

the result follows.

In words, the fraction of clique partitions of $K_{n}$ in which two given vertices belong to different subsets of the partition tends to 1 . The fraction of clique partitions satisfying a given triangle inequality at equality tends to 0 , as is witnessed by the following theorem.

Theorem 5.5 Let $K_{n}=\left(V_{n}, E_{n}\right)$ be the complete graph on $n$ vertices, let $i, j, k \in V_{n}$ be three distinct vertices, and let $a^{T} x \leq a_{0}$ denote the triangle inequality $x_{i j}+x_{i k}-x_{j k} \leq 1$. Then

$$
\lim _{n \rightarrow \infty} S_{1}\left(a, P\left(K_{n}\right)\right)=0
$$

Proof Let $A \subseteq E_{n}$ be a clique partition of $K_{n}$. The incidence vector of $A$ satisfies $x_{i j}+x_{i k}-x_{j k}=1$ if and only if $\{i, j\} \in A$ or $\{i, k\} \in A$. If both edges belong to $A$, then also $\{j, k\} \in A$, and the number of clique partitions containing the triangle $E_{n}(\{i, j, k\})$ is equal to $B_{n-2}$, since this triangle can be contracted to one vertex. Hence, the number of clique partitions $x \in P\left(K_{n}\right)$ satisfying $x_{i j}+x_{i k}-x_{j k}=1$ is equal to $2 B_{n-1}-B_{n-2}$, and

$$
S_{1}\left(a, P\left(K_{n}\right)\right)=\frac{2 B_{n-1}-B_{n-2}}{B_{n}}
$$


This fraction tends to 0 if $n \rightarrow \infty$.

In fact, the arguments used in the proof of Theorem 5.5 can be generalized, obtaining the following corollary.

Corollary 5.6 Let $K_{n}=\left(V_{n}, E_{n}\right)$ be the complete graph on $n$ vertices, and let $a^{T} x \leq a_{0}$ be a valid inequality for $P\left(K_{n}\right)$ with $a_{0}>0$. Then

$$
\lim _{n \rightarrow \infty} S_{1}\left(a, P\left(K_{n}\right)\right)=0
$$

Proof All clique partitions $A \subseteq E_{n}$ with $a^{T} \chi^{A}=a_{0}$ contain at least one edge (since $a_{0}>0$ ), and the number $d_{n}(a)$ of such clique partitions can be written as

$$
d_{n}(a)=\sum_{k=1}^{p} \lambda_{k} \cdot B_{n-k}
$$

for some fixed $p \in \mathbb{N}$ and some numbers $\lambda_{1}, \ldots, \lambda_{p} \in \mathbb{Z}$. Dividing $d_{n}(a)$ by $B_{n}$ and taking the limit for $n \rightarrow \infty$ gives the result.

Corollary 5.6 says that Definition 5.1 does not provide a good measure for the strength of an inequality when considering the clique partitioning polytope, although this measure may be well suited for studying the strength of valid inequalities for other polyhedra. In the sequel of this chapter we compute the strength (according to the other two definitions of Section 5.2) of the facets of the clique partitioning polytope with respect to the linear programming relaxation.

Computing the strength of the inequality $c^{T} x \leq c_{0}$ with respect to the polytope $P_{L P}\left(K_{n}\right)$ asks for optimizing $c^{T} x$ over $P_{L P}\left(K_{n}\right)$. This requires a linear programming formulation for $\max \left\{c^{T} x \mid x \in P_{L P}\left(K_{n}\right)\right\}$ and its dual.

The linear programming relaxation of (2.1) for the complete graph $K_{\pi}=$ $\left(V_{n}, E_{n}\right)$ reads

$$
\begin{array}{lll}
\text { Maximize } & \sum_{\{i, j\} \in E_{n}} c_{i j} x_{i j} & \\
\text { Subject to } & x_{i j}+x_{i k}-x_{j k} \leq 1 & \forall i, j, k \in V_{n} \\
& x_{i j} \geq 0 & \forall\{i, j\} \in E_{n}
\end{array}
$$


The dual problem of (5.3) is given by

$$
\begin{array}{lll}
\text { Minimize } & \sum_{\{i, j\} \in E_{n}} \sum_{k \in V_{n} \backslash\{i, j\}} y_{i j}^{k} & \\
\text { Subject to } & \sum_{k \in V_{i n} \backslash\{i, j\}}-y_{i j}^{k}+y_{i k}^{j}+y_{j k}^{i} \geq c_{i j} & \forall\{i, j\} \in E_{n} \\
& y_{i j j}^{k} \geq 0 & \forall i, j, k \in V_{n}
\end{array}
$$

where $y_{i j}^{k}$ is the dual variable corresponding to the triangle inequality $-x_{i j}+$ $x_{i k}+x_{j k} \leq 1$. We denote (5.3) by $\max \left\{c^{T} x \mid A x \leq b, x \geq 0\right\}$ and (5.4) by $\min \left\{b^{T} y \mid A^{T} y \geq c, y \geq 0\right\}$. By strong duality (see Chvátal [19])

$$
\max \left\{c^{T} x \mid A x \leq b, x \geq 0\right\}=\min \left\{b^{T} y \mid A^{T} y \geq c, y \geq 0\right\}
$$

This enables us to check if a given feasible solution for (5.3) is optimal.

In each of the following theorems we determine the strength of a class of facets of $P\left(K_{n}\right)$ with respect to the polytope $P_{L P}\left(K_{n}\right)$.

Theorem 5.7 Let $K_{n}=\left(V_{n}, E_{n}\right)$ be a complete graph, let $S, T \subseteq V_{n}$ be two nonempty disjoint sets, $1 \leq|S|<|T|$, and let $c^{T} x \leq c_{0}$ denote the 2-partition inequality (3.1) induced by $S$ and $T$. Then $S_{2}\left(c_{,} P_{L P}\left(K_{n}\right)\right)=\frac{1}{2}|T|$.

Proof Let $x \in \mathbb{R}^{\left|E_{n}\right|}$ be given by

$$
x_{i j}= \begin{cases}\frac{1}{2} & \text { if }\{i, j\} \in E_{n}(S, T) \\ 0 & \text { otherwise }\end{cases}
$$

Then $x$ is a feasible solution to $\max \left\{c^{T} x \mid A x \leq b, x \geq 0\right\}$, in fact it is an optimal solution. In order to prove this, we need to construct a dual feasible solution with the same optimal value. If $|T|$ is even, let $M_{1} \subseteq E_{n}(T)$ be a perfect matching in the (complete) graph induced by $T$; if $|T|$ is odd, let $M_{2} \subseteq E_{n}(T)$ be a triangle, and let $M_{1} \subseteq E_{n}(T)$ be a perfect matching in the 
graph induced by $T \backslash V\left(M_{2}\right)$. Construct a dual feasible solution $y \in \mathbb{R}^{3\left(\begin{array}{c}n \\ 3\end{array}\right)}$ as follows.

$$
y_{i j}^{k}= \begin{cases}1 & \text { if }\{i, j\} \in M_{1}, k \in S \\ \frac{1}{2} & \text { if }\{i, j\} \in M_{2}, k \in S \\ 0 & \text { otherwise }\end{cases}
$$

One can easily verify that $y$ is a dual feasible solution and $c^{T} x=b^{T} y$. Hence

$$
S_{2}\left(c, P_{L P}\left(K_{n}\right)\right)=\frac{\frac{1}{2}|S||T|}{|S|}=\frac{|T|}{2}
$$

For the strength according to Definition 5.3 of a 2-partition inequality with respect to $P_{L P}\left(K_{n}\right)$ we have the following corollary.

Corollary 5.8 Let $K_{n}=\left(V_{n}, E_{n}\right)$ be a complete graph, let $S, T \subseteq V_{n}$ be two nonempty disjoint sets, $1 \leq|S|<|T|$, and let $c^{T} x \leq c_{0}$ denote the 2-partition inequality (3.1) induced by $S$ and $T$. Then

$$
S_{3}\left(c, P_{L P}\left(K_{n}\right)\right)=\frac{\frac{1}{2}|S||T|-|S|}{\sqrt{(|S|+|T|)}}
$$

Proof Maximizing $c^{T} x$ over $P_{L P}\left(K_{n}\right)$ yields $\frac{1}{2}|S||T|, c_{0}=|S|$, and $\|c\|^{2}=$ $\left({ }_{2}|S|+|T|\right)$. The corollary follows from combining these results.

Theorem 5.9 Let $K_{n}=\left(V_{n}, E_{n}\right)$ be a complete graph, let $d \geq 2$ be an integer, let $s \in V_{n}, T \subseteq V_{n} \backslash\{s\}$ with $|T| \geq d+1$, and let $c^{T} x \leq c_{0}$ denote the claw inequality

$$
d \cdot x\left(E_{n}(\{s\}, T)\right)-x\left(E_{n}(T)\right) \leq\left(\begin{array}{c}
d+1 \\
2
\end{array}\right)
$$

Then $S_{2}\left(a, P_{L P}\left(K_{n}\right)\right)=\frac{|T|}{d+1}$. 
Proof Define $x \in \mathbb{R}^{\mathbb{E}}\left|E_{n}\right|$ by

$$
x_{i j}= \begin{cases}\frac{1}{2} & \text { if }\{i, j\} \in E_{n}(\{s\}, T) \\ 0 & \text { otherwise }\end{cases}
$$

Furthermore, define $y \in \mathbb{R}^{3\left(\begin{array}{c}n \\ 3\end{array}\right)}$ by

$$
y_{i j}^{k}= \begin{cases}\frac{d}{|T|-1} & \text { if }\{i, j\} \in E_{n}(T), k=s \\ 0 & \text { otherwise }\end{cases}
$$

One can easily verify that $x$ is a feasible solution to (5.3), and that $y$ is a dual feasible solution with $c^{T} x=b^{T} y$. Hence

$$
S_{2}\left(c, P_{L P}\left(K_{n}\right)\right)=\frac{\frac{1}{2} d|T|}{\left(\begin{array}{c}
d+1 \\
2
\end{array}\right)}=\frac{|T|}{d+1}
$$

Corollary 5.10 Let $K_{n}=\left(V_{n}, E_{n}\right)$ be a complete graph, let $d \geq 2$ be an integer, let $s \in V_{n}, T \subseteq V_{n} \backslash\{s\}$ with $|T| \geq d+1$, and denote the claw inequality $(5.5)$ by $c^{T} x \leq c_{0}$. Then

$$
S_{3}\left(c, P_{L P}\left(K_{n}\right)\right)=\frac{\frac{1}{2} d|T|-\left(\begin{array}{c}
d+1 \\
2
\end{array}\right)}{\sqrt{d^{2}|T|+\left(\begin{array}{c}
|T| \\
2
\end{array}\right)}}
$$

Proof Straightforward.

It is straightforward to compute the strength according to Definition 5.3 if the strength according to Definition 5.2 is known. Therefore, in the remainder we do not consider the distance measure.

Theorem 5.11 Let $K_{n}=\left(V_{n}, E_{n}\right)$ be a complete graph, let $C \subseteq E_{n}$ be an odd cycle of length at least 5 , and let $\bar{C} \in E_{n}$ be the set of 2-chords of $C$. The strength of the 2-chorded cycle inequality

$$
x(C)-x(\bar{C}) \leq\left\lfloor\frac{1}{2}|C|\right\rfloor
$$

with respect to the polytope $P_{L P}\left(K_{n}\right)$ is equal to $\frac{|C|}{|C|-1}$. 
Proof Denote the 2-chorded cycle inequality by $c^{T} x \leq c_{0}$, and let $x \in \mathbb{R}^{\left|E_{n}\right|}$ be given by $x_{i j}=\frac{1}{2}$ for all $\{i, j\} \in C$ and $x_{i j}=0$ otherwise. Clearly, $x$ is a feasible solution to (5.3). But, since the left-hand side of the 2-chorded cycle inequality induced by $C$ is the sum of $|C|$ triangle inequalities, each with weight $\frac{1}{2}, x$ is also the optimal solution to $(5.3)$. This proves that

$$
S_{2}\left(c, P_{L P}\left(K_{n}\right)\right)=\frac{\frac{1}{2}|C|}{\frac{1}{2}(|C|-1)}=\frac{|C|}{|C|-1}
$$

Theorem 5.12 Let $K_{n}=\left(V_{n}, E_{n}\right)$ be a complete graph, let $C \subseteq E_{n}$ be an odd cycle of length at least 3 , and let $z \in V_{n}$ be a vertex not incident to $C$. The strength of the wheel inequality

$$
x\left(E_{n}(\{z\}, V(C))\right)-x(C) \leq\left\lfloor\frac{1}{2}|C|\right\rfloor
$$

with respect to the polytope $P_{L P}\left(K_{n}\right)$ is equal to $\frac{|C|}{|C|-1}$.

Proof Let $x \in \mathbb{R}^{\left|E_{n}\right|}$ be given by $x_{i j}=\frac{1}{2}$ for all $\{i, j\} \in E_{n}(\{z\}, V(C))$ and $x_{i j}=0$ otherwise. Clearly, $x$ is a feasible solution to (5.3). But, since the left-hand side of the wheel inequality induced by $z$ and $C$ is the sum of $|C|$ triangle inequalities, each with weight $\frac{1}{2}, x$ is also the optimal solution to (5.3). This proves that

$$
S_{2}\left(c, P_{L P}\left(K_{n}\right)\right)=\frac{\frac{1}{2}|C|}{\frac{1}{2}(|C|-1)}=\frac{|C|}{|C|-1}
$$

Theorem 5.13 Let $K_{n}=\left(V_{n}, E_{n}\right)$ be a complete graph, let $C \subseteq E_{n}$ be an odd cycle of length at least 3 , and let $z_{1}, z_{2} \in V_{n}$ be two vertices not incident to $C$. The strength of the bicycle wheel inequality

$$
x\left(E_{n}\left(\left\{z_{1}, z_{2}\right\}, V(C)\right)\right)-x(C) \leq|C|-1
$$

with respect to the polytope $P_{L P}\left(K_{n}\right)$ is equal to $\frac{|C|}{|C|-1}$. 
Proof Denote the bicycle wheel inequality by $c^{T} x \leq c_{0}$, and let $x \in \mathbb{R}^{\mid E_{n}} \mid$ be given by $x_{i j}=\frac{1}{2}$ for all $\{i, j\} \in E_{n}\left(\left\{s_{1}, s_{2}\right\}, V(C)\right)$ and $x_{i j}=0$ otherwise. Then $x$ is an optimal solution to $\max \left\{c^{T} x \mid x \in P_{L P}\left(K_{n}\right)\right\}$, because the dual feasible solution $y$ given by $y_{i j}^{k}=\frac{1}{2}$ for all $\{i, j\} \in C, k \in\left\{s_{1}, s_{2}\right\}$ and $y_{i j}^{k}=0$ otherwise has the same objective value. Hence

$$
S_{2}\left(c, P_{L P}\left(K_{n}\right)\right)=\frac{|C|}{|C|-1}
$$

Theorem 5.14 Let $K_{n}=\left(V_{n}, E_{n}\right)$ be a complete graph, let $z \in V_{n}$, and let $P=\left\{\left\{u_{i}, u_{i+1}\right\} \mid i=1, \ldots, p\right\}$ be an even path in $K_{n}$ not incident to $z$. The strength of the 2-chorded path inequality (3.3) induced by $P$ and $z$ with respect to $P_{L P}\left(K_{n}\right)$ is $\frac{3}{2}$.

Proof Let $c^{T} x \leq c_{0}$ denote the 2-chorded path inequality, and let $x \in \mathbb{R}^{|E|}$ be given by $x_{i j}=\frac{1}{2}$ for all $\{i, j\} \in E_{n}$ with $c_{i j}=1$ and $x_{i j}=0$ for all other edges. Since $0 \leq x_{i j} \leq \frac{1}{2}$ for all $\{i, j\} \in E_{n}$ it is obvious that $x$ is a feasible

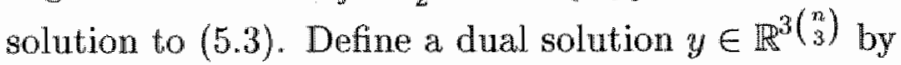

$$
y_{i j}^{k}= \begin{cases}\frac{1}{2} \quad \text { if } k \in\left\{u_{2}, u_{4}, \ldots, u_{p-1}\right\} \text { and } \\ & \{i, j\} \in\left\{\left\{z, u_{k-1}\right\},\left\{z, u_{k+1}\right\},\left\{u_{k-1}, u_{k+1}\right\}\right\} \\ 0 & \text { otherwise }\end{cases}
$$

One can verify that $y$ is a feasible solution to (5.4), and that $c^{T^{*}} x=b^{T} y$. Hence

$$
S_{2}\left(c, P_{L P}\left(K_{n}\right)\right)=\frac{3 \cdot \frac{1}{2} \cdot \frac{1}{2}(p-1)}{\frac{1}{2}(p-1)}=\frac{3}{2}
$$

Theorem 5.15 Let $K_{n}=\left(V_{n}, E_{n}\right)$ be a complete graph, let $z \in V_{n}$, and let $C \subseteq E_{n}$ be an even cycle in $K_{n}$ not incident to $z$. The strength of the 2-chorded wheel inequality induced by $C$ and $z$ with respect to $P_{L P}\left(K_{n}\right)$ is $\frac{3}{2}$.

Proof Analogous to the proof the Theorem 5.14. $\square$ 
Theorem 5.16 Let $K_{n}=\left(V_{n}, E_{n}\right)$ be a complete graph, let $s \in V_{n}$, and let $H=(U, F)$ be a subgraph of $K_{n}$ with $s \notin U$. If there exists a vector $w \in \mathbb{R}_{+}^{|F|}$ such that

$$
\sum_{j \in U:\{i, j\} \in F} w_{i j}=1 \quad \forall i \in U
$$

then the stable set inequality $x\left(E_{n}(\{s\}, U)\right)-x(F) \leq \alpha(H)$ has strength $\frac{\left|U^{\prime}\right|}{2 \alpha(H)}$ with respect to $P_{L P}\left(K_{n}\right)$.

Proof Denote the stable set inequality $x\left(E_{n}(\{s\}, U)\right)-x(F) \leq \alpha(H)$ by $c^{T} x \leq c_{0}$, and let $x \in \mathbb{R}^{\left|E_{n}\right|}$ be given by $x_{i j}=\frac{1}{2}$ for all $\{i, j\} \in E_{n}(\{s\}, U)$ and $x_{i j}=0$ otherwise. Since $0 \leq x_{i j} \leq \frac{1}{2}$ for all $\{i, j\} \in E_{n}$ it is obvious that $x$ is a feasible solution to (5.3), and $c^{T} x=\frac{1}{2}|U|$.

Let $w \in \mathbb{R}_{+}^{|F|}$ be a vector satisfying (5.6). Define a dual solution $y \in \mathbb{R}^{3\left(\begin{array}{l}n \\ 3\end{array}\right)}$ by

$$
y_{i j}^{k}= \begin{cases}w_{i j} & \text { if }\{i, j\} \in F, k=s \\ 0 & \text { otherwise }\end{cases}
$$

One can verify that $y$ is a feasible solution to (5.4) and

$$
\sum_{\{i, j\} \in E_{n}} \sum_{k \in V_{n} \backslash\{i, j\}} y_{i j}^{k}=\frac{1}{2}|U|
$$

Hence

$$
S_{2}\left(c, P_{L P}\left(K_{n}\right)\right)=\frac{|U|}{2 \alpha(H)}
$$

Remark 5.17 If $H$ contains a perfect matching $M \subseteq F$, then we can take $w \in \mathbb{R}_{+}^{|F|}$ with $w_{i j}=1$ for all $\{i, j\} \in M$ and $w_{i j}=0$ otherwise. If $H$ contains a Hamilton cycle $C \in F$, then we can take $w \in \mathbb{R}_{+}^{\mid F !}$ with $w_{i j}=\frac{1}{2}$ for all $\{i, j\} \in C$ and $w_{i j}=0$ otherwise. All facet inducing graphs $H$ we know contain a perfect matching or a Hamilton cycle. 


\section{Chapter 6}

\section{Separation}

\subsection{Introduction}

Solving a combinatorial optimization problem by polyhedral methods requires a (partial) description of the structure of the corresponding polyhedron $P$. In case a complete description is known the problem can be solved in polynomial time, that is, polynomial in the size of the complete description. However, in most situations only a partial description of the polyhedron is known, which provides a relaxation $R$ of $P$. Extreme points of this relaxation are not necessarily extreme points of the original polyhedron, but deciding whether a vertex of $R$ is also a vertex of $P$ is usually very easy. It comes down to checking if the vertex of the relaxation corresponds to a feasible solution of the combinatorial problem. If so, then one has a complete description of the polyhedron $P$ at this vertex. If not, then there is a facet defining hyperplane for $P$ separating this polyhedron and the given vertex of the relaxation. Although deciding whether such a hyperplane exists is easy, finding one is of a different matter. This so-called separation problem can be formulated as follows.

Given a polyhedron $P \subseteq \mathbb{R}^{n}$, a class $C$ of facets of $P$, and a point $x \in \mathbb{R}^{n}$. Find a facet (hyperplane) in $C$ separating $x$ and $P$, or prove that no such hyperplane exists.

For some polyhedra and some classes of facets the complexity of separation is known. For instance, the separation problem for the class of triangle inequalities for the clique partitioning problem can be solved in polymomial time, 
since the number of acets in this class is polymomialy bounded. But even if at chass contains exponentially many might be easy. The separation problen associated with the class of subtour elimination constraints for the travelug salesman problem 134,35 can be solved efficitly, athough this class contains exponentially many facets. On the other hand, the separation problem for the class of clique constraints for the set packing polyhedra [60] is NP-hard, since it requires the identification of a maximum weighted clique.

In this chapter we consider the complexty of separation for some classes of facets of the cligue partitioning polytope. We prove that the separation problem for the class of facets whin right-hand side 1 is NP-hard, as well as the separation problem for the claw inequalites. Next to these "negative' results, we show that a vartant of the separation problem for the composed 2-partition inequalites is solvable in polynomial time. Moreover, we discuss the separation problem for the class of (bicycle) wheel inequalities.

\subsection{Separation}

In Chapters 2-5 we described several new classes of facet defining inequalities for the clique partitioning polytope. Whether these facets are valuable for solving instances of clique partitioning by means of a branch-and-cut algorithm depends on a number of issues. For instance, how 'good' are the facets with respect to the current relaxation? We provided an answer to this question in Chapter 5 , where we defined three different measures for the strength of an inequality. Another issue is the complexity of separation, that is, given a graph $G=(V, E)$ and a (fractional) solution $x \in \mathbb{R}^{|E|}$, decide whether there exists a hyperplane separating $x$ and $P(G)$, and if so, find one. In this section we consider the complexity of separation for several classes of facets for the clique partitioning polytope.

We formulate our separation results in terms of complete graphs. The reason for this is twofold. First, every clique partition of a graph $G=(V, E)$ with $|V|=n$ can also be seen as a clique partition of the complete graph $K_{n}$. Similarly, every point $x \in \mathbb{R}^{|E|}$ can be transformed to a point $x^{\prime} \in \mathbb{R}^{\left|E_{n}\right|}$ by adding an appropriate number of zeros. Hence, we might as well consider complete graphs. Second, dealing with incomplete graphs requires a number 
of technicalities in our proofs, which do not provide any additional insight in the separation problem, but instead make the proofs harder to read.

\subsubsection{2-partition inequalities}

The 2-partition inequalities for the polytope $P\left(K_{n}\right)$ are given by (3.1). In Section 3.5 we proved that the class of 2-partition inequalities contains all facets of $P\left(K_{n}\right)$ with right-hand side 1 . The separation problem for this subclass of facets can be formulated as follows.

SEPRHS1:

Given: a complete graph $K_{n}=\left(V_{n}, E_{n}\right)$, a vertex $s \in V_{\text {g }}$, and a (fractional) point $x \in \mathbb{R}_{+}^{\left|E_{n}\right|}$.

Question: is there a subset $T \subseteq V_{n} \backslash\{s\}$ such that

$$
x\left(E_{n}(\{s\}, T)\right)-x\left(E_{n}(T)\right)>1
$$

The following theorem shows that SEPRHS1 is NP-complete.

Theorem 6.1 Let $K_{n}=\left(V_{n}, E_{n}\right)$ be a complete graph, $s \in V_{n}$, and let $x \in$ $\mathbb{R}_{+}^{\left|E_{n}\right|}$. Finding a subset $T \subseteq V_{n} \backslash\{s\}$ such that (6.1) holds, or showing that no such subset exists is NP-hard.

Proof To prove the theorem we show that the corresponding decision problem. SEPRHS1 is NP-complete, by giving a transformation of MAXCUT (which is known to be NP-complete, see Garey and Johnson [31]). MAXCUT is defined as follows.

\section{MAXCUT:}

Given: a graph $G=(V, E)$, edge weights $w_{i j} \in \mathbb{N}$ for all $\{i, j\} \in E$, and an integer $K \in \mathbb{N}$.

Question: is there a partition of $V$ into two disjoint sets $V_{1}$ and $V_{2}$, such that

$$
\sum_{\{i, j\} \in E\left(V_{1}, V_{2}\right)} w_{i j}>K
$$


Given an instance of MAXCUT, construct an instance of SEPRHSI as follows: $K_{n}=\left(V_{n}, E_{n}\right)$ is the complete graph with vertex set $V_{n}=V \cup\{s\}$, and $x \in \mathbb{R}^{\mid E_{n} !} \mid$ is defined by

$$
x_{i j}= \begin{cases}\frac{1}{K} \cdot \sum_{\{j, k\} \in E} w_{j k} & \text { if } i=s, j \in V \\ \frac{2}{K} \cdot w_{i j} & \text { if }\{i, j\} \in E \\ 0 & \text { atherwise }\end{cases}
$$

Let $T \subseteq V$. Then, the following holds.

$$
\begin{aligned}
& \sum_{i \in T} x_{s i}-\sum_{\{i, j\} \in E_{i s}(T)} x_{i j}= \\
& =\frac{1}{K} \cdot \sum_{i \in T} \sum_{\substack{j \in V \\
\{i, j\} \in E}} w_{i j}-\frac{2}{K} \cdot \sum_{\{i, j\} \in E(T)} w_{i j} \\
& =\frac{1}{K} \cdot \sum_{i \in T}\left(\sum_{\substack{j \in T \\
\{i, j\} \in E}} w_{i j}+\sum_{j \in V \backslash T} w_{i j}\right)-\frac{2}{K} \cdot \sum_{\{i, j\} \in E(T)} w_{i j} \\
& =\frac{1}{K} \cdot \sum_{i \in T} \sum_{\substack{\{\in \in, j\} \in E \\
\{i, j\}}} w_{i j}+\frac{1}{K} \cdot \sum_{i \in T} \sum_{j \in V \backslash T} w_{i j}-\frac{2}{K} \cdot \sum_{\{i, j\} \in E} w_{i j} \\
& =\frac{2}{K} \cdot \sum_{\{i, j\} \in E(T)} w_{i j}+\frac{1}{K} \cdot \sum_{\{i, j\} \in E(T, V \backslash T)} w_{i, j}-\frac{2}{K} \cdot \sum_{\{i, j\} \in E(T)} w_{i j} \\
& =\frac{1}{K} \cdot \sum_{\{i, j\} \in E(T, V \backslash T)} w_{i j}
\end{aligned}
$$

Hence, there exists a $T \subseteq V$ satisfying $x\left(E_{n}(\{s\}, T)\right)-x\left(E_{n}(T)\right)>1$ if and only if there exists a partition of $V$ into $V_{1}$ and $V_{2}$, satisfying (6.2). This completes the proof of the theorem.

When solving instances of clique partitioning with a branch-and-cut algorithm, using the linear programming relaxation of (2.1) in combination with cutting planes, the obtained fractional solution $x \in \mathbb{R}^{\left|E_{n}\right|}$ satisfies all triangle inequalities. A natural question to ask is whether this knowledge makes the decision 
problem SEPRHS1 any easier. Unfortunately, this is not the case. To see this, consider MAXCUT with the following restrictions.

(i) $w_{i j}=1$ for all $\{i, j\} \in E$,

(ii) there is no subset $V_{1} \subseteq V$ with $\left|V_{1}\right| \leq 2$ satisfying (6.2),

(iii) all vertices in $V$ have degree at least 2 ,

(iv) $K \geq 4$.

It is well known that MAXCUT remains NP-complete if $w_{i j}=1$ for all $\{i, j\} \in E$ (see again Garey and Johnson [31]). Checking if there exists a subset $V_{1} \subseteq V$ with $\left|V_{1}\right| \leq 2$ such that the cut $E\left(V_{1}, V \backslash V_{1}\right)$ has value more than $K$ can be done in polynomial time. Hence, MAXCUT with restrictions (i) and (ii) is still NP-complete. Restriction (iii) is no real restriction. If there is a vertex $i \in V$ with degree 1 , say that $\{i, j\} \in E$ is the only edge incident to this vertex, and this edge does not belong to the cut, then moving vertex $i$ to 'the other side' of the cut increases the value of the cut by 1 . Hence, node $i$ and edge $\{i, j\}$ can be deleted from $G$, if at the same time $K$ is replaced by $K-1$ (remember that all edge weights are equal to 1 ).

For fixed $K \in \mathbb{N}$, instances of MAXCUT with all edge weights equal to 1 can be solved in $O\left(|E|^{K}\right)$ time. Hence, instances of MAXCUT with restrictions (i)(iii) and $K \leq 3$ can be solved in polynomial time. However, MAXCUT with restrictions (i)-(iii) is NP-complete, and therefore it is also NP-complete if restrictions (i)--(iv) hold.

With these restrictions, it can be shown that the vector $x \in \mathbb{R}^{\left|E_{n}\right|}$ satisfies all triangle inequalities. Let $i, j \in V$ be two distinct vertices. If $\{i, j\} \in E$, then the triangle inequality $x_{s i}+x_{s j}-x_{i j} \leq 1$ is equivalent to

$$
\frac{1}{K} \cdot \sum_{\substack{k \in V \\\{i, k\} \in E}} w_{i k}+\frac{1}{K} \cdot \sum_{\substack{k \in V \\\{j, k\} \in E}} w_{j k}-\frac{2}{K} \cdot w_{i j} \leq 1
$$

The left-hand side of $(6.3)$ is exactly $\frac{1}{K}$ times the value of the cut $E\left(V_{1}, V_{2}\right)$ with $V_{1}=\{i, j\}$, and due to restriction (ii) this cut has value at most $K$. Hence, $x$ satisfies the triangle inequality $x_{s i}+x_{s j}-x_{i j} \leq 1$. 
The triangle inequality $x_{s i}-x_{s j}+x_{i j} \leq 1$ is equivalent to

$$
\frac{1}{K} \cdot \sum_{\substack{k \in V \\\{i, k\} \in E}} w_{i k}-\frac{1}{K} \cdot \sum_{\substack{k \in V \\ 0, k\} \in E}} w_{j k}+\frac{2}{K} \cdot w_{i j} \leq 1
$$

Restrictions (ii) and (iii) imply

$$
\sum_{\substack{k \in V \\\{i, k\} \in E}} w_{i k} \leq K \quad \text { and } \quad \sum_{\substack{k \in V \\\{j, k\} \in E}} w_{j k} \geq 2
$$

This implies that inequality (6.4) is indeed satisfied. Now, let $k \in V, k \neq i, j$. If $\{i, j, k\}$ induces a triangle in $E$, then the triangle inequality $x_{i j}+x_{i k}-x_{j k} \leq 1$ is equivalent to

$$
\frac{2}{K} \cdot\left(w_{i j}+w_{i k}-w_{j k}\right) \leq 1
$$

If only the edges $\{i, j\}$ and $\{i, k\}$ belong to $E$, then the $K_{1,2}$-inequality $x_{i j}+$ $x_{i k} \leq 1$ is equivalent to

$$
\frac{2}{K} \cdot\left(w_{i j}+w_{i k}\right) \leq 1
$$

Notice that (6.5) is implied by (6.6), which in turn is implied by restrictions (i) and (iv). Hence, the vector $x \in \mathbb{R}^{\mid E_{n} !}$ satisfies all triangle inequalities. This proves that, given a complete graph $K_{n}=\left(V_{n}, E_{n}\right)$ and a vector $x \in \mathbb{R}^{\left|E_{n}\right|}$ satisfying all triangle inequalities, finding a 2-partition inequality with righthand side 1 separating $x$ and $P\left(K_{n}\right)$, or proving that no such inequality exists, is NP-hard.

\subsubsection{Claw inequalities}

The structure of the claw inequalities (3.7) resembles the structure of the 2partition inequalities with right-hand side 1 . In a similar fashion as before, we can prove that the separation problem for the claw inequalities is NP-complete. This separation problem can be formulated as follows. 


\section{SEPCLAW:}

Given: a complete graph $K_{n}=\left(V_{n}, E_{n}\right)$, a vertex $s \in V_{n}$, an integer $c \geq 2$, and a (fractional) point $x \in \mathbb{R}_{+}^{\left|E_{n}\right|}$.

Question: is there a subset $T \subseteq V_{n} \backslash\{s\}$, such that

$$
c \cdot x\left(E_{n}(\{s\}, T)\right)-x\left(E_{n}(T)\right)>\left(\begin{array}{c}
c+1 \\
2
\end{array}\right)
$$

We have the following theorem.

Theorem 6.2 Let $K_{n}=\left(V_{n}, E_{n}\right)$ be a complete graph, $s \in V_{n}, c \geq 2$ integer, and let $x \in \mathbb{R}^{\left|E_{n}\right|}$. Finding a subset $T \subseteq V_{n} \backslash\{s\}$ such that (6.7) holds, or proving that no such subset exists, is NP-hard.

Proof Again we prove the theorem by giving a transformation of MAXCUT to SEPCLAW. Given an instance of MAXCUT (i.e. given a graph $G=(V, E)$, edge weights $w_{i j} \in \mathbb{N}$ for all $\{i, j\} \in E$, and an integer $K \in \mathbb{N}$ ), construct an instance of SEPCLAW as follows: $K_{n}=\left(V_{n}, E_{n}\right)$ is the complete graph with vertex set $V_{n}=V \cup\{s\}$, and $x \in \mathbb{R}^{\left|E_{n}\right|}$ is defined by

$$
x_{i j}= \begin{cases}\frac{c+1}{2 K} \cdot \sum_{\{j, k\} \in E} w_{j k} & \text { if } i=s, j \in V \\ \frac{c(c+1)}{K} \cdot w_{i j} & \text { if }\{i, j\} \in E \\ 0 & \text { otherwise }\end{cases}
$$

Analogous to the proof of Theorem 6.1 we find

$$
c \cdot \sum_{i \in T} x_{s i}-\sum_{\{i, j\} \in E_{n}(T)} x_{i j}=\frac{1}{K} \cdot \sum_{\{i, j\} \in E(T, V \backslash T)} w_{i j}
$$

Thus a subset $T \subseteq V$ satisfying (6.7) exists if and only if there is a partition of $V$ into $V_{1}$ and $V_{2}$ satisfying $(6.2)$. 


\subsection{3 $\left\{0, \frac{1}{2}\right\}$-Chvátal-Gomory cuts}

Some classes of facets of an integer polyhedron $P$ can be deduced from a system of inequalities partially describing $P$, using only elementary algebra and not using knowledge of the underlying combinatorial problem. Consider the integer polyhedron $P:=\left\{x \in \mathbb{Z}^{n} \mid A x \leq b\right\}$, where $A \in \mathbb{Z}^{m \times n}$ and $b \in \mathbb{Z}^{m}$. A Chvátal-Gomory cut [16] is a valid inequality for $P$ of the form $\lambda^{T} A x \leq\left\lfloor\lambda^{T} b\right\rfloor$ for some $\lambda \in \mathbb{R}_{+}^{m}$ such that $\lambda^{T} A \in \mathbb{Z}^{n}$. Notice that a ChvátalGomory cut is valid by construction, not by definition! Moreover, without loss of generality $\lambda$ can be restricted to $\lambda \in[0,1)^{m}$. Adding all these cuts to the system of inequalities for $P$ results in a tighter description of $P$, and the procedure to obtain Chvátal-Gomory cuts can be applied repeatedly. The minimum number of iterations of this procedure to obtain a given inequality is called the (Chvátal-) rank [16] of this inequality. The rank of the integer linear program $A x \leq b, x \in \mathbb{Z}^{n}$, is the minimum number of iterations required to eliminate the integrality constraints.

Caprara and Fischetti [11] study $\left\{0, \frac{1}{2}\right\}$-Chvátal-Gomory cuts, i.e. cuts arising from $\lambda \in\left\{0, \frac{1}{2}\right\}^{m}$. For a polyhedron $P_{0}=\left\{x \in \mathbb{R}^{n} \mid A x \leq b\right\}$ the rank-1 closure is defined as

$$
P_{1}:=\left\{x \in P_{0} \mid \lambda^{T} A x \leq\left\lfloor\lambda^{T} b\right\rfloor, \lambda \in[0,1)^{m}, \lambda^{T} A \in \mathbb{Z}^{n}\right\}
$$

With the restriction $\lambda \in\left\{0, \frac{1}{2}\right\}^{m}$ we have

$$
P_{1 / 2}:=\left\{x \in P_{0} \mid \lambda^{T} A x \leq\left\lfloor\lambda^{T} b\right\rfloor, \lambda \in\left\{0, \frac{1}{2}\right\}^{m}, \lambda^{T} A \in \mathbb{Z}^{n}\right\}
$$

Notice that $P \subseteq P_{1} \subseteq P_{1 / 2} \subseteq P_{0}$, where $P$ is the convex hull of all integral points in $P_{0}$. The $\left\{0, \frac{1}{2}\right\}$-cut separation problem $\left(\left\{0, \frac{1}{2}\right\}\right.$-SEP $)$ reads: given a point $x \in P_{0}$, find $\lambda \in\left\{0, \frac{1}{2}\right\}^{m}$ such that $\lambda^{T} A x>\left[\lambda^{T} b\right]$ and $\lambda^{T} A x \in \mathbb{Z}^{n}$, or prove that no such $\lambda$ exists. Caprara and Fischetti prove that $\left\{0, \frac{1}{2}\right\}$-SEP is NP-hard in general, and that for some matrices $A \in \mathbb{Z}^{m \times n}$ the separation problem is solvable in polynomial time, for instance if $A$ contains at most 2 odd coefficients per row. This result implies for the clique partitioning polytope $P(G)$ that the 2-chorded cycle inequalities (3.2) and the wheel inequalities (3.5) can be separated in polynomial time [11]. The constraint matrix $A$ for the clique partitioning problem consists of the triangle inequalities, the 
$K_{1,2}$-inequalities and the nonnegativity constraints. The triangle inequality $x_{i j}+x_{i k}-x_{j k} \leq 1(\dot{i}, j, k \in V)$ induces a row in $A$ with 3 odd coefficients, but adding the nonnegativity constraint $-x_{j k} \leq 0$ to this inequality results in $x_{i j}+x_{i k}-2 x_{j k} \leq 1$. Now, consider a 2-chorded cycle inequality (3.2) induced by an odd cycle $C \subseteq E_{n}$ with $V_{n}(C)=\left\{v_{1}, \ldots, v_{k}\right\}$ (the constraints induced by even cycles are already implied by the triangle inequalities). The 2-chorded cycle inequality induced by $C$ is a $\left\{0, \frac{1}{2}\right\}$-cut obtained by combining the following constraints.

$$
x_{v_{i}, v_{i+1}}+x_{v_{i+1}, v_{i+2}}-2 x_{v_{i}, v_{i+2}} \leq 1 \quad \forall i=1, \ldots, k
$$

Thus the following theorem holds.

Theorem 6.3 [11] Let $K_{n}=\left(V_{n}, E_{n}\right)$ be a complete graph, and let $x \in \mathbb{R}^{\left|E_{n}\right|}$ be a point satisfying all basic inequalities. Finding an odd cycle $C \subseteq E_{n}$ such that

$$
x(C)-x(\bar{C})>\left\lfloor\frac{1}{2}|C|\right\rfloor
$$

where $\bar{C}=\mathrm{Ch}_{2}(C)$, or showing that no such cycle exists, can be done in polynomial time.

Muiller [56] gave a direct combinatorial algorithm for separating the 2-chorded cycle inequalities. In a very similar fashion it is shown that the wheel inequalities can also be separated in polynomial time. Deza et al. [25] present. an algorithm for separating the wheel inequalities for the multicut polytope $M C_{n} \leq\left(K_{n}\right)$. Since this polytope is nothing but an affine transformation of the clique partitioning polytope $P\left(K_{n}\right)$ their algorithm can easily be adjusted to an algorithm for separating the wheel inequalities for the clique partitioning polytope, hence we have the following theorem.

Theorem 6.4 [25] Let $K_{n}=\left(V_{n}, E_{n}\right)$ be a complete graph, and let $x \in \mathbb{R}^{\mid E_{n}} \mid$ be a point satisfying all basic inequalities. Finding an odd cycle $C \subseteq E_{n}$ and a. vertex $z \in V_{n}$ not incident to $C$ such that

$$
x\left(E_{n}\left(\{z\}, V_{n}(C)\right)\right)-x(C)>\left\lfloor\frac{1}{2}|C|\right\rfloor
$$

or showing that no such cycle and vertex exist, can be done in polynomial time. 


\subsubsection{Bicycle wheel inequalities}

Let $K_{n}=\left(V_{n}, E_{n}\right)$ be a complete graph, let $C \subseteq E_{n}$ be an odd cycle in $K_{n}$, and let $T=\left\{v_{1}, v_{2}\right\}$ be a set of two vertices in $V_{n}$ not incident to $C$. Define $A=E_{n}(T)$ and $S:=E_{n}\left(T, V_{n}(C)\right)$. The set $F:=C \cup A \cup S$ is a bicycle wheel in $K_{n}$ with $\operatorname{rim} C$, axle $A$ and spokes $S$. Barahona et al. [5] introduce the class of bicycle wheel inequalities

$$
x(F) \leq 2|C|
$$

for the bipartite subgraph polytope $P_{B}(G)$, and they prove that these inequalities define facets of $P_{B}(G)$ if all edges in $F$ belong to $G$. The bipartite subgraph polytope $P_{B}(G)$ contains the cut polytope $P_{C}(G)$, and therefore the bicycle wheel inequalities are also valid for $P_{C}(G)$. Moreover, Barahona and Mahjoub [6] prove that these inequalities are even facet defining for $P_{C}(G)$ if $G$ contains all edges in $F$. They also describe three methods to construct facets from facets. One of these methods is the so-called switching operation, given in the following theorem.

Theorem 6.5 (Switching [6]) Let $G=(V, E)$ be a graph, and let $a^{T} x \leq a_{0}$ be a facet defining inequality for $P_{C}(G)$. Let $v \in V$, and let $\delta(v)$ be the set of edges in $E$ incident to $v$. The inequality

$$
-\sum_{\{i, j\} \in \delta(v)} a_{i j} x_{i j}+\sum_{\{i, j\} \mathbb{E} \delta(v)} a_{i j} x_{i j} \leq a_{0}-a^{T} \chi^{\delta(v)}
$$

defines a facet of $P_{C}(G)$.

In words this theorem says that if in a facet defining inequality $a^{T} x \leq a_{0}$ the signs of all edges incident to a node $v \in V$ are changed and the right-hand side is lowered by $a^{T} \chi^{\delta(v)}$ a new facet defining inequality is obtained. Applying this theorem twice to the bicycle wheel inequality (6.8), first to $v_{1}$ and next to $v_{2}$, yields that

$$
x(C \cup A)-x(S) \leq 0
$$


defines a homogeneous facet of $P_{C}(G)$, i.e. a facet with right-hand side 0 . Remember that the cut polytope $P_{C}(G)$ and the multicut cut polytope $M C_{2}^{\leq}(G)$ are different names for the same polytope. In the case of complete graphs Deza et al. [25] show that all multicut polytopes $M C_{k}^{\leq}\left(K_{n}\right)$ with $2 \leq k \leq n$ have the same set of homogeneous facets, so the bicycle wheel inequalities (6.9) also define facets of $M C_{n}^{\leq}\left(K_{n}\right)$, which is nothing but an affine transformation of the clique partitioning polytope $P\left(K_{n}\right)$, since

$$
M C_{n}^{\leq}\left(K_{n}\right)=\left\{1-x \mid x \in P\left(K_{n}\right)\right\}
$$

Consequently, the inequalities

$$
x(S)-x(C \cup A) \leq|C|-1
$$

define facets of $P\left(K_{n}\right)$. Notice that (6.10) is just an alternative way of writing inequalities (3.6). Gerards [32] gave an algorithm for separating the bicycle wheel inequalities (6.8) for the bipartite subgraph polytope. This algorithm can of course also be applied to separate inequalities (6.8) for the cut polytope, and it also translates to an algorithm for separating the switched bicycle wheel inequalities (6.9), as noticed in Deza et al. [25]. In the sequel we reformulate Gerards' algorithm for the clique partitioning polytope. The separation problem for the bicycle wheel inequalities for the clique partitioning polytope is as follows.

BICYCLESEP:

Given: a complete graph $K_{n}=\left(V_{n}, E_{n}\right)$, and a fractional point $x \in \mathbb{R}^{\left|E_{n}\right|}$ satisfying all basic inequalities.

Question: is there an odd cycle $C \subseteq E_{n}$ and a set $S \subseteq V_{n}$ of two vertices not incident to $C$, such that

$$
x\left(E_{n}\left(S, V_{n}(C)\right)\right)-x\left(E_{n}(S)\right)-x(C)>|C|-1
$$

Theorem 6.6 shows that BICYCLESEP is solvable in polynomial time.

Theorem 6.6 [25] Let $K_{n}=\left(V_{n}, E_{n}\right)$ be a complete graph, and let $x \in \mathbb{R}\left|E_{n}\right|$ be a point satisfying all basic inequalities. Finding an odd cycle $C \subseteq E_{n}$ and a set $S=\left\{s_{1}, s_{2}\right\} \subseteq V_{n}$ such that (6.11) holds, or showing that no such cycle and set exist, can be done in polynomial time. 
Proof We prove the theorem by showing that finding a violated bicycle wheel inequality is equivalent to finding $\left|E_{n}\right|$ times a minimum weight odd cycle in a graph, which can be done in polynomial time (see Grötschel and Pulleyblank [36]).

Given $K_{n}=\left(V_{n}, E_{n}\right), S=\left\{s_{1}, s_{2}\right\} \subseteq V_{n}$, and $x \in \mathbb{R}^{\left|E_{n}\right|}$, construct a graph $G=(V, E)$ as follows: $V=V_{n} \backslash\left\{s_{1}, s_{2}\right\}, E=E_{n}(V)$, and each edge $\{i, j\} \in E$ has weight $w_{i j}=\frac{1}{2}\left(2+2 x_{i j}-x_{s_{1}, i}-x_{s_{\Perp}, j}-x_{s_{2}, i}-x_{s_{2}, j}\right)$. Notice that

$$
\begin{aligned}
w_{i j} & =\frac{1}{2}\left(2+2 x_{i j}-x_{s_{1, i}}-x_{s_{1}, j}-x_{s_{2}, i}-x_{s_{2}, j}\right) \\
& =\frac{1}{2}\left(1-\left(x_{s_{1}, i}+x_{s_{1}, j}-x_{i j}\right)+1-\left(x_{s_{2, i}, i}+x_{s_{2}, j}-x_{i j}\right)\right) \\
& \geq \frac{1}{2}(1-1+1-1) \geq 0
\end{aligned}
$$

since $x$ satisfies all basic inequalities.

Claim: A violated bicycle wheel inequality exists if and only if there is an odd cycle in $G$ with weight less then $1-x_{s_{1}, s_{2}}$.

Proof: Let $C \subseteq E_{n}$ be an odd cycle with vertex set $\left\{v_{1}, \ldots, v_{k}\right\}$. Then this cycle has weight

$$
\begin{aligned}
\sum_{i=1}^{k} w_{v_{i}, v_{i+1}} & =\frac{1}{2} \cdot \sum_{i=1}^{k}\left(2+2 x_{v_{i}, v_{i+1}}-x_{s_{1}, v_{i}}-x_{s_{1}, v_{i+1}}-x_{s_{2}, v_{i}}-x_{s_{2}, v_{i+1}}\right) \\
& =k+\sum_{i=1}^{k} x_{v_{i}, y_{i+1}}-\sum_{i=1}^{k}\left(x_{s_{1}, v_{i}}+x_{s_{2}, v_{i}}\right) \\
& =1+2\left\lfloor\frac{k}{2}\right\rfloor-\left(\sum_{i=1}^{k}\left(x_{s_{1}, v_{i}}+x_{s_{2}, v_{i}}\right)-\sum_{i=1}^{k} x_{v_{i}, v_{i+1}}\right)
\end{aligned}
$$

Thus (6.11) holds if and only if $C$ has weight less than $1-x_{s_{1}, s_{2}}$.

Remark 6.7 Although the structures of the wheel inequalities and the bicycle wheel inequalities are very much the same, the results of Caprara and Fischetti [11] do not imply that the odd bicycle wheel inequalities can be separatied in polynomial time, since the Chvatal rank of these inequalities is more than one. To see this, notice that the sum of the positive coefficients at the 
left-hand side of ( 6.10$)$ equals $2|C|$. In order to obtain a bicycle wheel inequality one has to combine a number of triangle inequalities with total weight at least $|C|$, but then the right-hand side of the obtained Chvattal-Gomory cut has right-hand side at least $|C|$, whereas it should be at most $|C|-1$.

\subsubsection{Composed 2-partition inequalities}

In Chapter 3 we described the disjoint patching procedure, a technique to construct facets from facets. One version of this procedure is disjoint $(S, T)$ patching (see Theorem 3.30), which takes as input two 2-partition inequalities with disjoint supports and right-hand side 1 , and delivers as output a new facet defining inequality for $P\left(K_{n}\right)$, called a composed 2-partition inequality. There are, however, exponentially many ways of patching the given 2-partition inequalities together. In Theorem 6.8 we prove that, given the two 2-partition inequalities and a point $x \in \mathbb{R}^{\left|E_{n}\right|}$, it can be decided in polynomial time whether there exists a disjoint $(S, T)$-patching of these inequalities separating $x$ and $P\left(K_{n}\right)$. This separation problem can be formulated as follows.

\section{SEPPATCH:}

Given: a complete graph $K_{n}=\left(V_{n}, E_{n}\right)$, two 2-partition inequalities $a^{T} x \leq 1$ and $b^{T} x \leq 1$ with support $\left(V_{a}, E_{a}\right)$ resp. $\left(V_{b}, E_{b}\right)$ and centers $s, t \in V_{n}$, such that $V_{a} \cap V_{b}=\varnothing$, and a (fractional) point $x \in \mathbb{R}_{+}^{\left|E_{m}\right|}$.

Question: is there a partition of $V_{a} \backslash\{s\}$ into $U_{a}$ and $W_{a}$ and a partition of $V_{b} \backslash\{t\}$ into $U_{b}$ and $W_{b}$ such that

$$
(a+b)^{T} x+x_{s t}-\sum_{j \in U_{b}} x_{s j}-\sum_{i \in U_{a}} x_{t i}-\sum_{\{i, j\} \in E\left(W_{a}, W_{b}\right)} x_{i j}>2
$$

The following theorem proves that SEPPATCH is solvable in polynomial time, by showing that it is equivalent to finding a minimum cut in a directed graph, which can found in polynomial time, see e.g. Ahuja et al. [2].

Theorem 6.8 SEPPATCH is solvable in polynomial time.

Proof We show that SEPPATCH is equivalent to finding a minimum cut in a directed graph. Given the 2-partition inequalities and the point $x \in \mathbb{R}^{E_{n} \mid}$ 
construct a digraph $D=\left(V^{\prime}, A\right)$ with $V^{\prime}=V_{a} \cup V_{b}$ and

$$
\begin{aligned}
A= & \left\{(s, u) \mid u \in V_{b} \backslash\{t\}\right\} \cup\left\{(v, t) \mid t \in V_{a} \backslash\{s\}\right\} \\
& \cup\left\{(u, v) \mid u \in V_{b} \backslash\{t\}, v \in V_{a} \backslash\{s\}\right\}
\end{aligned}
$$

The capacity of arc $(i, j) \in A$ is equal to $x_{i j}$. Rewriting (6.12) yields

$$
\sum_{j \in U_{b}} x_{s j}+\sum_{i \in U_{a}} x_{t i}+\sum_{\{i, j\} \in E\left(W_{a}, W_{b}\right)} x_{i j}<(a+b)^{T} x+x_{s t}-2
$$

The right-hand side of (6.13) is fixed, whereas the left-hand side is exactly the value of the $\{s, t\}$-cut $E_{n}\left(\{s\} \cup U_{a} \cup W_{b},\{t\} \cup U_{b} \cup W_{a}\right)$ in $D$ (see Figure 6.1). If the minimum $\{s, t\}$-cut in $D$ is less than $(a+b)^{T} x+x_{s t}-2$, then there exists a separating disjoint $(S, T)$-patching. Otherwise, no such patching exists.

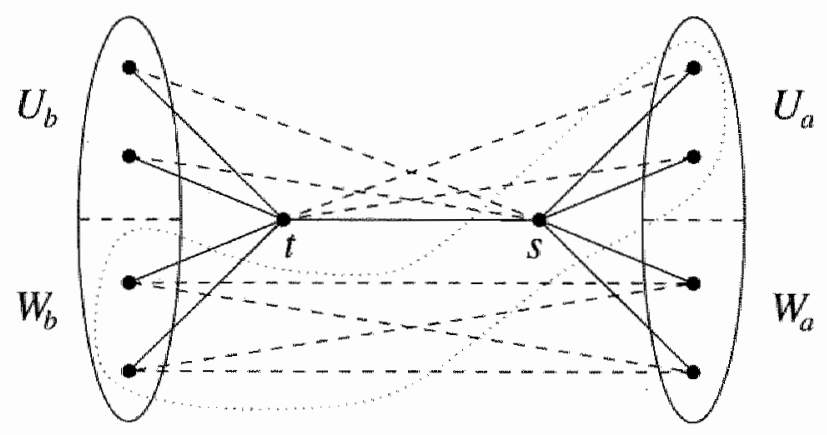

Figure 6.1: The correspondence between a disjoint $(S, T)$-patching and a cut in a digraph.

\section{Acknowledgements}

This chapter is based on joint work with Maarten Oosten and Frits Spieksma. 


\section{Chapter 7}

\section{Disconnecting graphs}

\subsection{Introduction}

The previous chapters all dealt with various aspects of the clique partitioning problem. In this chapter we consider a different, but related, problem that can be formulated as follows. Given a graph $G=(V, E)$ and an integer $c \geq 1$, remove as few vertices as possible such that no component of the resulting graph has more than $c$ vertices. More precisely, for $S \subseteq V$ define $\rho(S):=\{\{i, j\} \in E \mid i \in S, j \in V\}$, and find a subgraph $G^{\prime}=(V \backslash S, E \backslash \rho(S))$ of $G$ with $|S|$ as small as possible, such that no component of $G^{\prime}$ has more than $c$ vertices. Notice that for $c=1$ this is equivalent to the familiar independent set problem. So the problem of disconnecting graphs, referred to as $D G$, is a generalization of the independent set problem, and thereby it is NP-hard.

This problem arises in a variety of contexts. For instance, suppose that one is given a linear (integer) program with a corresponding constraint matrix $A \in \mathbb{R}^{m \times r_{b}}$ and some integer $d \geq 1$. Two rows of $A$ are said to be connected if and only if there exists a column for which both rows have a nonzero entry. One is interested in assigning as many rows of $A$ as possible to so-called blocks such that no more than $d$ rows are assigned to each block, and a pair of rows assigned to different blocks is not connected. When a constraint matrix can be brought into such a bordered block diagonal form, this may help the solution process of the associated linear (or integer) program. This interesting application of DG is described in Borndörfer et al. [9, 10] and Gupta [41] (see their papers and 
of not deleted vertices. It follows from the connectivity constraints that if such a pair of vertices is connected they are in a same component. Further, since these $x$-variables satisfy the triangle inequalities and the capacity constraints they constitute a partition of the associated vertex-set into components of no more than $c$ vertices. Let us refer to the convex hull of all feasible solutions to $(7.1)$ as $P(G, c)$.

Consider now another formulation for $\mathrm{DG}$, that has as an additional input parameter an integer $B \in \mathbb{N}$, which is an upper bound on the number of components to be formed. This formulation uses variables $z_{i b} \in\{0,1\}$ for all $i \in V$ and $b \in\{1, \ldots, B\}$, where $z_{i b}=1$ if vertex $i$ is assigned to component $b$, and $z_{i b}=0$ otherwise. The corresponding $\{0,1\}$-program, referred to as (DG2), reads as follows.

$$
\begin{array}{lll}
\text { Maximize } & \sum_{i \in V} \sum_{b=1}^{B} z_{i b} & \\
\text { Subject to } & \sum_{b=1}^{B} z_{i b} \leq 1 & \forall i \in V \\
& \sum_{i \in V} z_{i b} \leq c & \forall b \in\{1, \ldots, B\} \\
& z_{i b}+z_{j b^{\prime}} \leq \mathbb{1} & \forall\{i, j\} \in E, \\
& z_{i b} \in\{0,1\} & \forall i \in V, b^{\prime} \in\{1, \ldots, B\}, b \neq b^{\prime} \\
&
\end{array}
$$

The formulation (DG2) is proposed by Borndörfer et al. [9] in the context of decomposing constraints matrices, and by Kumar et al. [47] in a cell formation context. We denote the convex hull of all feasible solutions to (7.2) by $P(G, B, c)$. In order to be able to compare both formulations, take $B=n$ in (DG2), so that the problem of disconnecting graphs is addressed in both formulations. Let us now discuss some of the advantages and disadvantages of these formulations.

A first thing to notice is of course the number of constraints, which is $O\left(n^{3}\right)$ in (DG1) versus $O\left(n^{2}|E|\right)$ in (DG2), and the number of variables which is $m+n$ in (DG1) and $n^{2}$ in (DG2). This means that for connected graphs, which are the most interesting ones, (DG1) provides a more compact formulation.

Second, (DG2) suffers from symmetry. Indeed, for any solution satisfying (DG2) (or its linear programming relaxation) there are $B$ ! equivalent ones. 
This constitutes a potential hurdle when solving (DG2) using a branch-andcut algorithm, as reported in Borndörfer et al. [9]. See Section 7.4, Crama and Oosten [22] and Oosten [59] for more comments along this line.

Third, consider the optimal value of the LP-relaxation of both formulations, denoted by $v_{L P}^{D G 1}$ and $v_{L P}^{D G 2}$ respectively. It is not difficult to observe that, given a feasible solution $\left(x^{*}, y^{*}\right) \in \mathbb{R}^{m+n}$ to the LP-relaxation of (DG1), we can construct a feasible solution $z^{*} \in \mathbb{R}^{n^{2}}$ to the LP-relaxation of (DG2) with the same value by setting $z_{i b}^{*}=y_{i}^{*} / n$ for all $i \in V$ and $b=1, \ldots, n$. Moreover, a feasible solution to the LP-relaxation of (DG2) with value $n$ is found by setting $z_{i b}^{*}=1 / n$ for all $i \in V$ and $b=1, \ldots, n$. Summarizing, we have

$$
v_{L P}^{D G 1} \leq v_{L P}^{D G 2}=n
$$

For (DG1) the value of the LP-relaxation depends on the graph $G$ and the capacity. However, there exist instances for which the value of the LP-relaxation is strictly less than $n$.

Fourth, let us consider the number of integer variables. At first sight, (DG2) seems to be better in that respect, however we have the following lemma.

Lemma 7.1 Let $(x, y) \in \mathbb{R}^{m} \times \mathbb{R}^{n}$ be a feasible solution to the linear programming relaxation of (DG1) with $y_{i} \in\{0,1\}$ for all $i \in V$. Then there exists a feasible solution $(\bar{x}, \bar{y}) \in\{0,1\}^{m+n}$ to (DG1) with $\bar{y}_{i}=y_{i}$ for all $i \in V$.

Proof Notice that for distinct vertices $i, j, k \in V$ with $y_{i}=y_{j}=y_{k}=1$ and $\{i, j\},\{i, k\} \in E$ one has $x_{i j}=x_{i k}=1$ due to the connectivity constraints, and hence $x_{j k}=1$ due to triangle inequalities.

Let $G_{1}=\left(V_{1}, E_{1}\right)$ be the subgraph of $G$ induced by $V_{1}=\left\{i \in V \mid y_{i}=\mathbb{1}\right\}$. The observation above implies that $x_{i j}=1$ if $i, j \in V_{1}$ belong to the same component of $G_{1}$.

Hence, the solution $(\bar{x}, \bar{y}) \in \mathbb{R}^{m+n}$ defined by $\bar{y}_{i}=y_{i}$ for all $i \in V$ and $\breve{x}_{i j}=\left\lfloor x_{i j}\right\rfloor$ for all $i, j \in V, i \neq j$ is a feasible solution to (DG1).

Thus, by Lemma 7.1 we can relax the constraints $x_{i j} \in\{0,1\} \quad(i, j \in V, i \neq j)$ in (DG1) to $0 \leq x_{i j} \leq 1$, so that the number of binary variables in (DG1) can be reduced to $n$ compared to $n^{2}$ integer variables in (DG2). 
Finally, let us consider the potential of both formulations with respect to formulating more generall problems. Obviously, if a nontrivial upper bound is known for the number of components to be formed, (DG2) is a more appropriate formulation. On the other hand, in a situation where the objective function is more general than the one considered here (for instance by considering weights for the edges of $G$, see Ferreira et al. [29] for a problem of this nature), formulation (DG1) can be easily adapted to incorporate this, in contrast to (DG2). In fact, all polyhedral results derived in Section 7.3 for (DG1) remain valid in such a setting.

\subsection{Polyhedral results}

In this section we deal with the facial structure of $P(G, c)$. Apart from focusing on finding facet defining inequalities of $P(G, c)$ (Subsection 7.3.3), we mainly try to exploit the relationship of $P(G, c)$ with other, well studied, polytopes in order to be able to use existing results for these polytopes. This subject is dealt with in Subsection 7.3.2. In Subsection 7.3.1 we investigate some properties of $P(G, c)$.

Let us first make the following easy observation concerning the facial structure of $P(G, c)$. The polytope $P(G, 1)$ is (isomorphic to) the independent set polytope, and has dimension $n$. The polytope $P(G, c)$ with $c \geq 2$ is full dimensional, i.e. it has dimension $\frac{1}{2}|V|(|V|+1)$, since it contains the zero vector and all unit vectors. In the sequel we assume that $c \geq 2$.

Further, for $U \subseteq V$ and $F \subseteq\{\{i, j\} \mid \dot{i}, j \in V, i \neq j\}$, we denote by $\chi(U, F) \in\{0,1\}^{n+m}$ the characteristic vector corresponding to $U$ and $F$, that is $\chi(U, F)=1$ if either $q \in U$ or $q$ is associated with a pair of vertices in $F$, and $\chi_{q}(U, F)=0$ otherwise.

\subsubsection{Properties of the facial structure of $P(G, c)$}

In this subsection we state conditions showing when a facet defining inequality for $P(G, c)$ for some graph $G$ and capacity $c \geq 1$ is facet defining for other graphs and/or other capacities. The next theorem shows that facet defining inequalities for $P(G, c)$ that are valid for subgraphs or increasing capacity remain facet defining. 
Theorem 7.2 Let $G=(V, E)$ be an arbitrary graph, $c \geq 2$ an integer, and let $(a, b)^{T}(x, y) \leq a_{0}$ be a facet defining inequality for $P(\bar{G}, c)$.

(i) If, for any $d \geq c$, the inequality $(a, b)^{T}(x, y) \leq a_{0}$ is valid for $P(G, d)$, then $(a, b)^{T}(x, y) \leq a_{0}$ is facet defining for $P(G, d)$.

(ii) If, for any $E^{\prime} \subseteq E$, the inequality $(a, b)^{T}(x, y) \leq a_{0}$ is valid for $P\left(\left(V, E^{\prime}\right), c\right)$, then $(a, b)^{T}(x, y) \leq a_{0}$ is facet defining for $P\left(\left(V, E^{\prime}\right), c\right)$.

Proof Since $(a, b)^{T}(x, y) \leq a_{0}$ is facet defining for $P(G, c)$, there exists a set $M$ of $\operatorname{dim}(P(G, c))$ affinely independent solutions satisfying $(a, b)^{T}(x, y)=a_{0}$. All solutions in $M$ are also contained in $P(G, d)$ and $P\left(G^{\prime}, c\right)$, and these two polytopes have the same dimension as $P(G, c)$ (since $c \geq 2$ ). Hence validity of $(a, b)^{T}(x, y) \leq a_{0}$ is sufficient.

The following theorem gives sufficient conditions to lift a facet defining inequality for $P\left(G^{\prime}, c\right)$ to a facet defining inequality for $P(G, d)$ for $d \geq c$, where $G^{\prime}$ is a vertex-induced subgraph of $G$.

Theorem 7.3 Let $G=(V, E)$ be a graph, let $c \geq 2$ be an integer, let $G^{\prime}=$ $(U, E(U))$ be a subgraph of $G$, and let

$$
\sum_{\substack{i, j \in U \\ i \neq j}} a_{i j} x_{i j}+\sum_{i \in U} b_{i} y_{i} \leq a_{0}
$$

be a facet defining inequality for $P\left(G^{\prime}, c\right)$ such that the following conditions hold.

(i) Inequality (7.3) is valid for $P(G, d)$ for some integer $d \geqq c$.

(ii) There exists an order $\left(e_{1}, \ldots, e_{p}\right)$ of the elements in $\tilde{E}:=\{(i, j\} \mid i \in$ $U, j \in V \backslash U\}$ such that for each $e_{k} \in \tilde{E}$ there exists $(x, y) \in P(G, d)$ satisfying (7.3) at equality with $x_{e_{k}}=1$ and $x_{e_{l}}=0$ for all $l=k+$ $1, \ldots, p$.

(iii) For all $i \in V \backslash U$ there exists $(x, y) \in P(G, d)$ with $y_{i}=1$ satisfying (7.3) at equality.

Then (7.3) is facet defining for $P(G, d)$. 
Proof Since (7.3) defines a facet of $P\left(G^{\prime}, c\right)$ there exists a set $M_{1}$ of $\frac{1}{2}|U|(|U|+$ 1) affinely independent solutions satisfying this inequality at equality. Let $\chi_{0}$ be the incidence vector of one of the solutions in $M_{1}$. Obviously, the following incidence vectors belong to $P(G, d)$ and satisfy $(7.3)$ at equality.

$$
\chi_{0}+\chi(\varnothing,\{i, j\}) \quad \forall i, j \in V \backslash U, i \neq j
$$

By condition (ii) there exists, for each $e_{k} \in \tilde{E}$, a point $\chi^{k}:=(x, y) \in P(G, d)$ with $(a, b)^{T}(x, y)=a_{0}, x_{e_{k}}=1$ and $x_{e_{l}}=0$ for all $l=k+1, \ldots, p$. The set $M_{2}:=\left\{\chi^{1}, \ldots, \chi^{p}\right\}$ consists of $p$ affinely independent points. Further, by condition (iii), for all $i \in V \backslash U$ there exists a vector $(x, y) \in P(G, d)$ with $y_{i}=1$ satisfying (7.3) at equality. These vectors, together with the ones described by (7.4) and the sets $M_{1}$ and $M_{2}$, yield exactly $\frac{1}{2}|V|(|V|+1)$ affinely independent solutions in $P(G, d)$ satisfying (7.3) at equality.

We end this subsection with some easy-to-prove properties of $P(G, c)$.

Theorem 7.4 Let $G=(V, E)$ be a graph, let $c \geq 2$ be an integer and let $(a, b)^{T}(x, y) \leq a_{0}$ be any facet defining inequality of $P(G, c)$.

(i) The inequalities $x_{i j} \geq 0(i, j \in V, i \neq j)$ define facets of $P(G, c)$.

(ii) The inequalities $x_{i j} \leq 1,(i, j \in V, i \neq j)$ do not define facets of $P(G, c)$.

(iii) The inequalities $y_{i} \geq 0(i \in V)$ define facets of $P(G, c)$.

(iv) The inequalities $y_{i} \leq 1(i \in V)$ define facets of $P(G, c)$.

(v) $a_{0} \geq 0$.

(vi) $b_{i} \geq 0$ for all $i \in V$.

Proof (i) The zero vector and all unit vectors but one satisfy $x_{i j}=0(i, j \in V$, $i \neq j$ ) and are affinely independent.

(ii) The inequality $x_{i j} \leq 1$ is the sum of the triangle inequalities $x_{i j}+x_{i k}-x_{i j} \leq$ 1 and $x_{i j}-x_{i k}+x_{i j} \leq 1$, and hence it is not facet defining.

(iii) The zero vector and all unit vectors but one satisfy $y_{i}=0(i \in V)$ and are affinely independent.

(iv) The following vectors satisfy $y_{i}=1$ and are linearly independent.

$$
\begin{aligned}
& \chi(\{i\}, \varnothing) \\
& \chi(\{i\},\{j, k\}) \quad \text { for all } j, k \in V, j \neq k \\
& \chi(\{i, j\},\{j, k\}) \text { for all } j \in V, j \neq i \text {. }
\end{aligned}
$$


(v) $P(G, c)$ contains the zero vector, hence $0=(a, b)^{T}(0,0) \leq a_{0}$.

(vi) Suppose that $b_{i}<0$ for some $i \in V$. Since $P(G, c)$ is full dimensional there exists a point $(x, y) \in P(G, c)$ with $(a, b)^{T}(x, y)=a_{0}$ and $y_{i}=1$. The point $(\bar{x}, \vec{y})$ obtained from $(x, y)$ by changing $y_{i}$ from 1 to 0 also belongs to $P(G, c)$, and $(a, b)^{T}(\bar{x}, \bar{y})>a_{0}$, a contradiction.

\subsubsection{Ties with other polytopes}

In this subsection we study ties between $P(G, c)$ and related polytopes. We prove that under certain conditions facets of the (capacitated) clique partitioning polytope, facets of the maximal weighted clique polytope, and facets of $P(G, B, c)$ each give rise to facets of $P(G, c)$. Let us first describe these polytopes in the case of complete graphs. The clique partitioning polytope $P\left(K_{n}\right)$ is the convex hull of all solutions to the integer linear program (2.1) given on page 22 . When imposing a bound on the number of vertices in each clique (say $c \geq 2$ ), one arrives at the capacitated clique partitioning problem: add to (2.1) the inequalities

$$
\sum_{j \in V_{n} \backslash\{i\}} x_{i j} \leq c-1 \quad \forall i \in V
$$

The capacitated clique partitioning polytope, denoted as $P_{\mathrm{CapCPP}}\left(K_{n}, c\right)$, is studied in Faigle et al. [27] (this polytope is called the $c$-restricted partition polytope in Dijkhuizen and Faigle [26], see also Section 1.5). When one is interested in finding a single maximal weighted clique containing at most $c$ vertices, one needs to add the inequalities (7.5) and

$$
x_{i j}+x_{j k}+x_{k l}-x_{i k}-x_{j l} \leq 1 \quad \forall \text { distinct } i, j, k, l \in V
$$

The clique polytope, denoted as $P_{\text {Clique }}\left(K_{n}, c\right)$, is studied by Dijkhuizen and Faigle [26]. An extended formulation for this problem is given by Park et al. [62], and by Macambira and de Souza [52].

Given a graph $G=(V, E)$ on $n$ vertices, one could also add to the formulation for $P_{\text {CapcPp }}\left(K_{n}, c\right)$ the variables $y_{i} \in\{0,1\}$ for all $i \in V$ and the connectivity constraints. This results in the polytope $P(G, c)$. 
Consider now $P(G, B, c)$. Given a vertex $z \in P(G, B, c)$ it is straightforward to construct a vertex $(x, y) \in \mathbb{R}^{m+n}$ of $P(G, c)$. Set

$$
y_{i}=\sum_{b=1}^{B} z_{i b}
$$

and $x_{i j}=1(i, j \in V, i \neq j)$ if there exists $b \in\{1, \ldots, B\}$ such that $z_{i b}=$ $z_{j b}=1$, and $x_{i j}=0$ otherwise. Notice that different vertices of $P(G, B, c)$ which correspond to permutations of the components all give rise to the same vertex of $P(G, c)$. This observation implies that valid inequalities $a^{T} z \leq a_{0}$ for $P(G, B, c)$ with $a_{i b}=a_{i b^{\prime}}$ for each $i \in V$ and all $b, b^{\prime} \in\{1, \ldots, B\}$ can be turned into valid inequalities for $P(G, c)$ by substituting (7.7) for all $i \in V$. Let us refer to inequalities for $P(G, B, c)$ with $a_{i b}=a_{i b^{r}}$ for all $i \in V$ and all $b, b^{\prime} \in\{1, \ldots, B\}$ as block invariant inequalities (see Borndörfer et al. [10]). Thus a block invariant inequality can be written as

$$
\sum_{i \in V} a_{i} \sum_{b=1}^{B} z_{i b} \leq a_{0} .
$$

Let us now summarize the relationships between the various polytopes introduced.

Lemma 7.5 (Inheritance of valid inequalities)

(i) An inequality valid for $P\left(K_{n}\right)$ is valid for $P_{\text {CapCPP }}\left(K_{n}, c\right)$ for all $c \in \mathbb{N}$.

(ii) An inequality valid for $P_{\text {CapCPp }}\left(K_{n}, c\right)$ is valid for $P_{\text {Clique }}\left(K_{n}, c\right)$ as well as valid for $P(G, c)$ for all $c \in \mathbb{N}$.

(iii) An inequality valid for $P_{\text {Clique }}\left(K_{n}, c\right)$ is valid for $P(G, c)$ if $G$ is a clique, for all $c \in \mathbb{N}$.

(iv) A block invariant inequality (7.8) valid for $P(G, B, c)$ is valid for $P(G, c)$ when substituting (7.7) for all $i \in V$.

\section{Proof Straightforward.}

A graphical representation of Lemma 7.5 is given in Figure 7.1.

Of course, a more powerful result would concern the facetness of inequalities instead of their validity. Given a facet defining inequality $a^{T} x \leq a_{0}$ of $P\left(K_{n}\right)$ 


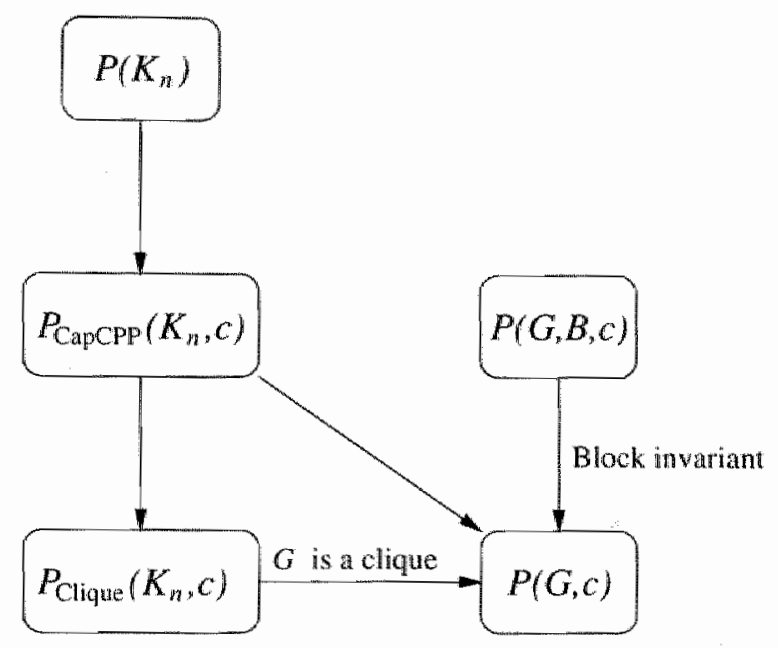

Figure 7.1: Inheritance of valid inequalities.

there exists a set $M_{a} \subseteq P\left(K_{n}\right)$ of $\operatorname{dim}\left(P\left(K_{n}\right)\right)$ affinely independent solutions satisfying this inequality at equality. Denote by $k\left(M_{a}\right)$ the size of the largest clique (in terms of number of vertices) present in a solution in $M_{a}$, and let $k_{a} \in \mathbb{N}$ be the minimum value of $k\left(M_{a}\right)$ over all affinely independent sets $M_{a} \subseteq P\left(K_{n}\right)$ with $\left|M_{a}\right|=\operatorname{dim}\left(P\left(K_{n}\right)\right)$ and $a^{T} x=a_{0}$ for all $x \in M_{a}$.

\section{Lemma 7.6 (Inheritance of facets)}

(i) An inequality $a^{T} x \leq a_{0}$ facet defining for $P\left(K_{n}\right)$ is also facet defining for $P_{\text {Capcpp }}\left(K_{n}, c\right)$ for all $c \geq k_{a}$.

(ii) An inequality $a^{T} x \leq a_{0}$ facet defining for $P_{\mathrm{CapCPP}}\left(K_{n}, c\right)$ is facet defining for $P(G, c)$ for all graphs $G=(V, E)$ with $|V|=n$.

(iii) A block invariant inequality

$$
\sum_{i \in V} b_{i} \sum_{b=1}^{B} z_{i b} \leq b_{0}
$$

facet defining for $P(G, B, c)$ is facet defining for $P(G, c)$ when substituting (7.7) for all $i \in V$.

Proof (i) Let $M_{a}$ be a maximal set of affinely independent solutions such that $k\left(M_{a}\right)=k_{a}$. Then $M_{a} \subseteq P_{\mathrm{CapCPP}}\left(K_{n,} c\right)$ for $c \geq k_{a}$, and since $P\left(K_{n}\right)$ 
and $P_{\text {Capcpp }}\left(K_{n}, c\right)$ have the same dimension it follows that $a^{T} x \leq a_{0}$ defines a facet of $P_{C a p C P P}\left(K_{n}, c\right)$.

(ii) Let $M$ be a maximal set of affinely independent solutions in the polytope $P_{\text {CapCpP }}\left(K_{n}, c\right)$, and let $\chi_{0}$ be the incidence vector of one of the solutions in $M$. The set $M$ together with the set $\left\{\chi_{0}+\chi(\{i\}, \varnothing) \mid i \in V\right\}$ is still affinely independent and has cardinality $\operatorname{dim}(P(G, c))$.

(iii) Consider inequalities (7.9) with substitution (7.7) for all $i \in V$. Let $F:=\left\{(x, y) \in P(G, c) \mid(0, b)^{T}(x, y)=b_{0}\right\}$ and suppose that $F$ is not a facet of $P(G, c)$. Then there exist $(p, q) \in \mathbb{R}^{m+n}$ and $p_{0} \in \mathbb{R}$ such that

$$
\sum_{\substack{i, j \in V \\ i \neq j}} p_{i j} x_{i j}+\sum_{i \in V} q_{i} y_{i}=p_{0}
$$

for all $(x, y) \in F$. Consider now, for all $(x, y) \in F$, all pairs of distinct vertices $i, j \in V$. Two situations can occur.

(iii.a) There exists $(x, y) \in F$ with $y_{i}+y_{j} \leq 1$. Then $p_{i j}=0$, because the solution with $x_{i j}=0$ as well as the solution with $x_{i j}=1$ are in $F$.

(iii.b) All $(x, y) \in F$ have $y_{i}=y_{j}=1$. This implies that the face induced by $(7.9)$ is contained in the hyperplane

$$
\sum_{b=1}^{B}\left(z_{i b}+z_{j b}\right)=2
$$

a contradiction, since (7.9) defines a facet of $P(G, B, c)$. Hence $p_{i j}=0$ for all $i, j \in V_{2} i \neq j$. Thus (7.10) boils down to

$$
\sum_{i \in V} q_{i} y_{i}=p_{0}
$$

which is satisfied by all $(x, y) \in F$, and hence

$$
\sum_{i \in V} q_{i} \sum_{b=1}^{B} z_{i b}=p_{0}
$$

holds for all $z \in P(G, B, c)$ satisfying (7.9) at equality. Thus, $\left(p, q, p_{0}\right)=$ $\beta \cdot\left(a, b, a_{0}\right)$ for some $\beta>0$, and we conclude that (iii) holds. 


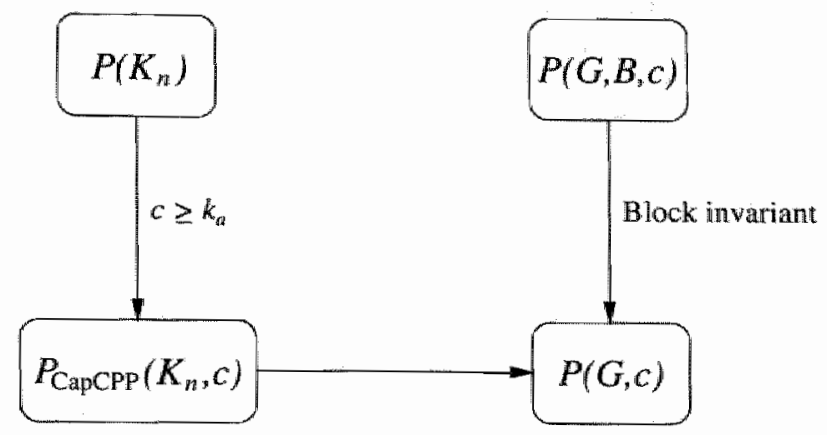

Figure 7.2: Inheritance of facet defining inequalities.

A graphical representation of Lemma 7.6 is given in Figure 7.2.

Lemma 7.6 gives us quite some information concerning the facetness of inequalities for $P(G, c)$. Using this lemma and the results described in $[10,27,39]$ and Chapter 3 , one can conclude for a number of classes of inequalities that they are facet defining for $P(G, c)$ for some $c \geq 2$. Here, we mention some examples listed as corollaries.

Corollary 7.7 Consider the class of circuit inequalities defined by Faigle et al. [27]. Let $C=\left\{v_{1}, \ldots, v_{c+1}\right\} \subseteq V$. The corresponding circuit inequality is given by

$$
x_{v_{1}, v_{2}}+x_{v_{2}, v_{3}}+\ldots+x_{v_{c+1}, w_{1}} \leq c-1
$$

These inequalities define facets of $P(G, c)$.

Proof This follows from Lemma 7.6 and the fact proven in [27] that these inequalities define facets of $P_{\mathrm{CapCPP}}\left(K_{n}, c\right)$.

Corollary 7.8 Consider the class of the 2-partition inequalities introduced by Grötschel and Wakabayashi [39]. Let $S, T \subseteq V$ be two disjoint subsets of vertices. The corresponding 2 -partition inequality is given by

$$
\sum_{i \in S} \sum_{j \in T} x_{i j}-\sum_{\substack{i, j \in S \\ i \neq j}} x_{i j}-\sum_{\substack{i, j \in T \\ i \neq j}} x_{i j} \leq \min \{|S|,|T|\}
$$


These inequalities define facets of $P(G, c)$ for all $c \geq 4$ if and only if $|S| \neq|T|$.

Proof This follows from Lemma 7.6, the proof in [39] that these inequalities define facets of $P\left(K_{7 b}\right)$ and the observation that $k_{a} \leq 4$, where $a^{T} x \leq a_{0}$ denotes inequality (7.11).

Notice that Corollary 7.8 implies that the triangle inequalities (which occur in the LP-relaxation of (DG1)) are facet defining for $P(G, c)$.

Borndörfer et al. [10] describe several classes of valid and facet defining block invariant inequalities for $P(G, B, c)$. Here we list only those classes which are used in our branch-and-cut algorithm (see Section 7.4).

Let $W \subseteq V$ with $|W|=c+k$ be such that the induced subgraph $(W, E(W))$ of $G$ is $k$-vertex connected. Then the inequality

$$
\sum_{i \in W} \sum_{b=1}^{B} z_{i b} \leq c
$$

is valid for $P(G, B, c)$. It is facet defining for $P(G, B, c)$ if and only if for each $i \notin W$ there exists some vertex cut $N$ in $(W \cup\{i\}, E(W \cup\{i\}))$ of size $k$ with $i \notin N$ (see [10]). Hence, we have the following corollary.

Corollary 7.9 Let $G=(V, E)$ be a graph, $c \geq 2$ an integer, and let $W \subseteq V$ with $|W|=c+k$ be such that $(W, E(W))$ is $k$-vertex connected. Then the generalized cover inequality

$$
\sum_{i \in W} y_{i} \leq c
$$

is valid for $P(G, c)$. It defines a facet if for all $i \notin W$ there exists some vertex cat $N$ in $\{W \cup\{i\}, E(W \cup\{i\}))$ of size $k$ with $i \notin N$.

Proof This follows from Lemma 7.6 and the proof in [10] that these inequalities define facets of $P(G, B, c)$.

In a similar fashion we can deduce the following two corollaries. 
Corollary 7.10 Let $G=(V, E)$ be a graph, $c \geq 2$ an integer, and let $W \subseteq$ $V$ be such that $(W, E(W))$ is a complete subgraph of $G$. Then the clique inequality

$$
\sum_{i \in W} y_{i} \leq c
$$

is valid for $P(G, c)$. It defines a facet if $|W| \geq c+1$ and for each vertex $i \notin W$ there exists $U \subseteq W$ with $|U|=c$, such that $i$ is not adjacent to any vertex in $U$.

Notice that the condition in Corollary 7.10 implies maximality of the clique $W$.

Corollary 7.11 Let $G=(V, E)$ be a graph, $c \geq 2$ an integer, and let $i \in V$ be such that $|N(i)| \geq c$. Then the star inequality

$$
(|N(i)|-c+1) \cdot y_{i}+\sum_{j \in N(i)} y_{j} \leq|N(i)|
$$

is valid for $P(G, c)$.

\subsubsection{More facets of $P(G, c)$}

Apart from the connectivity constraints, all inequalities we have seen so far deal exclusively with $x$-variables (when 'inherited' from $P_{\operatorname{Cap} C p p}\left(K_{n}, c\right)$ or $P\left(K_{n}\right)$ ) or exclusively with $y$-variables (when 'inherited' from $P(G, B, c)$ ). Here we derive facet defining inequalities containing both types of variables.

Theorem 7.12 Let $G=(V, E)$ be a graph, let $c \geq 2$ be an integer, and let the subgraph $(U, E(U))$ be a clique in $G$. Then the inequality

$$
\sum_{i \in U} y_{i}-\sum_{i, j \in U} x_{i j} \leq 1
$$

is valid and facet defining for $P(G, c)$. 
Proof Let $i_{0} \in U$. The following solutions satisfy (7.16) at equality and are linearly independent.

$\begin{array}{ll}\chi(\{i\}, \varnothing) & \forall i \in U \\ \chi(\{i, j\},\{i, j\}) & \forall i, j \in U, i \neq j \\ \chi(\{i\},\{i, j\}) & \forall i \in U, j \notin U \\ \chi\left(\left\{i_{0}, j\right\},\left\{i_{0}, j\right\}\right) & \forall j \notin U \\ \chi\left(\left\{i_{0}\right\},\{i, j\}\right) & \forall i, j \notin U, i \neq j\end{array}$

Notice that Theorem 7.12 implies that the connectivity constraints in (DG1) define facets of $P(G, c)$ for $c \geq 2$. Using Lemma 7.6 and Theorem 7.12 we can characterize all facet defining inequalities with right-hand side 1.

Theorem 7.13 Let $G=(V, E)$ be a graph and let $c \geq 4$ be an integer. All facet defining inequalities of $P(G, c)$ with integral coefficients and right-hand side 1 are given by the inequalities $y_{i} \leq 1$ for $i \in V$, the 2-partition inequalities (7.11) with $|S|=1$, and inequalities (7.16).

Proof We prove the theorem by showing that any inequality $(a, b)^{T}(x, y) \leq 1$ valid for $P(G, c)$ is implied by one of the inequalities mentioned above.

Obviously, $b_{i} \leq 1$ for all $i \in V$. Let $B=\left\{i \in V \mid b_{i}=1\right\}$. For each pair $i, j \in B$ we have $\{i, j\} \in E$ (otherwise there exists a solution that violates the inequality). So $B$ corresponds to the vertices of a clique in $G$. We distinguish three cases:

(i) $|B| \geq 2$. Obviously, $a_{i j} \leq 0$ if $i \notin B$ or $j \notin B$ (or both). Consider any edge $\{i, j\} \in E$ with $i, j \in B$. Then $b_{i j} \leq-1$, since otherwise we take $y_{i}=y_{j}=x_{i j}=1$ and all other variables 0 , which would violate the inequality. Thus in this case, a valid inequality is implied by (7.16).

(ii) $|B|=1$. For any pair of distinct vertices $i, j \in V$ we have $a_{i j} \leq 0$. Hence, a valid inequality of this form is implied by the upper bound constraints $y_{i} \leq 1$ $(i \in B)$.

(iii) $B=\varnothing$. Obviously, $a_{i j} \leq 1$ for all distinct $i, j \in V$. Moreover, any 2 edges with coefficient 1 must have a vertex in common, say vertex $s \in V$. Let $T=\left\{i \in V \mid a_{s i}=1\right\}$, then $a_{i j} \leq-1$ for all distinct $i, j \in T$ (otherwise consider $x_{s i}=x_{s j}=x_{i j}=1$ and all other variables 0 ). It follows that a valid inequality of this form is implied by the 2-partition inequalities with $|S|=1$. 
Inequalities (7.16) can be generalized as follows.

Theorem 7.14 Let $G=(V, E)$ be a graph, let $c, p \geq 2$ be two integers, and let $U \subseteq V$ be such that $(U, E(U))$ is a clique in $G$. Then the inequality

$$
p \cdot \sum_{i \in U} y_{i}-\sum_{\{i, j) \in E(U)} x_{i j} \leq\left(\begin{array}{c}
p+1 \\
2
\end{array}\right)
$$

is valid for $P(G, c)$. It defines a facet if and only if $|U| \geq p+2$.

Proof Validity: For fixed $p$, the left-hand side of (7.17) is maximal if either $p$ or $p+1$ vertices from $U$ are assigned to a component, and in that case the left-hand side and the right-hand side of (7.17) are equal.

Facet defining: If $|U| \leq p-1$ there are no solutions that satisfy (7.17) at equality. If $|U|=p$ all solutions that satisfy (7.17) at equality have all vertices from $U$ assigned to a component. If $|U|=p+1$ then (7.17) is nothing but the sum of the inequalities $y_{i}+y_{j}-x_{i j} \leq 1$ for all $i_{i} j \in U, i \neq j$. Now suppose what $|U| \geq p+2$, denote $(7.17)$ by $(a, b)^{T}(x, y) \leq a_{0}$, and let $(q, r)^{T}(x, y) \leq q_{0}$ be a facet inducing inequality such that $F_{a b}:=\left\{(x, y) \|(a, b)^{T}(x, y)=a_{0}\right\} \subseteq$ $F_{q r}:=\left\{(x, y) \mid(q, r)^{T}(x, y)=q_{0}\right\}$. We prove that $F_{a b}=F_{q r r}$ by showing that $a_{0} \cdot(q, r)=q_{0} \cdot(a, b)$. Similar arguments as used in the proof of Lemma 3.8 imply that

$$
\begin{aligned}
q_{i} & =\xi \cdot a_{i} \quad \forall i \in U \\
r_{i j} & =\xi \cdot b_{i j} \quad \forall\{i, j\} \in E(U)
\end{aligned}
$$

where $\xi:=\frac{q_{0}}{a_{0}}$. It remains to show that all other entries of $(q, r)$ are equal to zero. Let $U^{\prime} \subseteq U$ with $\left|U^{\prime}\right|=p, u \in U \backslash U^{\prime}$, and let $k, l \in V \backslash U, k \neq l$. It is obvious that $q_{k l}=0$. Define $F:=\left\{\{i, j\} \mid i, j \in U^{\prime}, i \neq j\right\}$. The solutions $\chi\left(U^{\prime}, F\right)$ and $\chi\left(U^{\prime}, F \cup\{\{u, i\}\}\right)$ both satisfy (7.17) at equality, and hence $q_{u i}=0$. Define $F^{\prime}:=\left\{\{i, j\} \mid i, j \in U^{\prime} \cup\{k\}, i \neq j\right\}$. Comparing $\chi\left(U^{\prime}, F\right)$ and $\chi\left(U^{\prime} \cup\{k\}, F^{\prime}\right)$ yields $r_{k}=0$.

Let us finally provide three classes of facet defining inequalities related to specific structures in the graph $G$. 
Theorem 7.15 Let $G=(V, E)$ be a graph, let $c \geq 3$ be an integer, and let $i, j, k \in V$ be such that $\{i, j\},\{i, k\} \in E$ and $\{j, k\} \notin E$. Then the inequality

$$
y_{i}+y_{j}+y_{k}-x_{j k} \leq 2
$$

is valid and facet defining for $P(G, c)$.

Proof Obviously, the inequality is valid. The following six solutions are linearly independent and satisfy (7.18) at equality.

$$
\begin{array}{ll}
\chi(\{i, j\},\{i, j\}) & \chi(\{j, k\},\{i, j\}) \\
\chi(\{i, k\},\{i, k\}) & \chi(\{j, k\},\{i, k\}) \\
\chi(\{j, k\}, \varnothing) & \chi(\{i, j, k\}, E(\{\{i, j, k\}))
\end{array}
$$

Consider all pairs of distinct vertices $l, m \in V \backslash\{i, j, k\}$. The following incidence vectors satisfy (7.18) at equality, and each incidence vector has a nonzero entry at a position where all the previous incidence vectors had a zero entry.

$$
\begin{aligned}
& \chi(\{i, j\},\{\{i, j\},\{i, l\}\}) \\
& \chi(\{i, j\},\{\{i, j\},\{j, l\}\}) \\
& \chi(\{i, k\},\{\{i, k\},\{k, l\}\}) \\
& \chi(\{i, k, l\}, E(\{i, k, l\}))
\end{aligned}
$$

Together this yields $\operatorname{dim}(P(G, c))$ linearly independent solutions satisfying (7.18) at equality.

We will refer to these inequalities as $K_{1,2}$-inequalities.

Theorem 7.16 Let $G=(V, E)$ be a graph, let $c \geq 3$ an integer, and let $U:=\{i, j, k, l\} \subseteq V$ be such that $(U, E(U))$ is a clique in $G$. Then the arrow inequality (see Figure 7.3)

$$
y(\{i, j, l\})+x_{i k}-x(E(\{j, k, l\})) \leq 2
$$

is valid and facet defining for $P(G, c)$. 


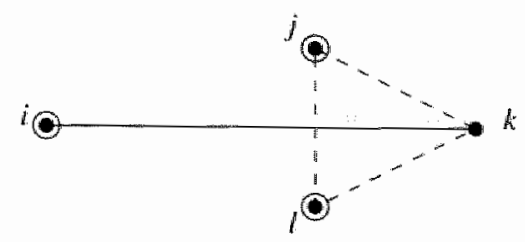

Figure 7.3: The supporti of an arrow inequality.

Proof It is easy to check that (7.19) is facet defining for $P\left(K_{4}, 3\right)$ and valid for $P(G, c)$. In order to prove that the arrow constraints define facets of $P(G, c)$ whenever $K_{4}$ is a subgraph of $G$ we use Theorem 7.3 . For each $m \in V \backslash U$ set

$$
\begin{aligned}
& \chi_{m}^{1}:=\chi(\{i, l\},\{\{i, l\},\{j, m\}\}) \\
& \chi_{m}^{2}:=\chi(\{i, l\},\{\{i, l\},\{k, m\}\}) \\
& \chi_{m}^{3}:=\chi(\{i, j\},\{\{i, j\},\{l, m\}\}) \\
& \chi_{m}^{4}:=\chi(\{i, j\}, E(\{i, j, m\}))
\end{aligned}
$$

and define $M_{2}:=\left\{\chi_{m}^{1}, \ldots, \chi_{m}^{4} \mid m \in V \backslash U\right\}$. Further, define

$$
M_{3}:=\{\chi(\{i, j, m\}, E(\{i, j, m\})) \mid m \in V \backslash U\}
$$

The set $M_{2}$ shows that condition (ii) of Theorem 7.3 is fulfilled, and $M_{3}$ shows that condition (iii) holds. Hence, the arrow constraints define facets of $P(G, c)$.

Theorem 7.17 Let $G=(V, E)$ be a graph, let $c \geq 3$ be an integer, and let $C:=\left\{v_{1}, v_{2}, v_{3}, v_{4}\right\}$ be a subset of $V$ that induces a cycle in $G$, and let $F:=\left\{\left\{v_{1}, v_{3}\right\},\left\{v_{2}, v_{4}\right\}\right\}$. Then the 4-cycle inequality

$$
y(C)-x(F) \leq 2
$$

is valid and facet defining for $P(G, c)$.

Proof Straightforward when using Theorem 7.3 . 


\subsection{Computational results}

In this section we show results from computational experiments. In Subsection 7.4.1 we compare formulations (DG1) and (DG2) from a computational point of view. Subsection 7.4 .2 presents results from a branch-and-cut algorithm that we implemented using results of Section 7.3. Finally, Subsection 7.4 .3 deals with the effect of the capacity on the number of components.

\subsection{1 (DG1) versus (DG2)}

Suppose one is given some instance of DG and a Mixed Integer Programming (MIP) solver, what would be the most promising formulation to choose, (DG1) or (DG2)? In order to answer this question we selected a set of instances and used the default MIP solver within CPLEX 2.1.

Recall that we described in Section 7.1 that the problem of bringing a constraint matrix into bordered block diagonal form reduces to the problem of disconnecting graphs. Borndörfer et al. [10] took a subset of the constraint matrices in the MIPLIB and NETLIB problem libraries as their test set. We use the same test here and restricted ourselves to the 10 smallest instances in the MrPLiB library (see Table 7.1 and 7.2). The first four columns describe the name of the problem, and the values of $|V|,|E|$ and $c$, respectively. Each instance was solved twice, once using formulation (DG1) and once using formulation (DG2). Each time at most one hour of computing time was devoted to solving a formulation. Column 5 gives the number of nodes in the branching tree that was considered by the MIP solver. Column 6 gives the computing time, column 7 gives the best solution found, and the last column gives the best known upper bound after one hour of computing time. An asterisk in Table 7.2 indicates that the computation was stopped because of insufficient memory to store information concerning the nodes in the branch-and-bound tree.

From these experiments it is clear that formulation (DG2) indeed suffers badly from symmetry. Using this formulation, only instances mod008 and ffugpl are solved within an hour of computing time, whereas using formulation (DG1) all instances except rgn are solved. 


\begin{tabular}{|l|rrr|rrrr|}
\hline Name & $|\bar{V}|$ & $|E|$ & $c$ & \multicolumn{2}{|c|}{ (DG1) } & \multicolumn{3}{c|}{ Incumbent } \\
& & & & Nodes & Time & Obj. & Ub. \\
\hline mod008 & 6 & 15 & 4 & 4 & $00: 00$ & 4 & 4 \\
stein9 & 13 & 66 & 7 & 27 & $00: 15$ & 7 & 7 \\
p0040 & 13 & 30 & 7 & 5 & $00: 04$ & 10 & 10 \\
p0033 & 15 & 40 & 8 & 19 & $00: 46$ & 12 & 12 \\
gt1 & 15 & 61 & 8 & 10 & $00: 31$ & 10 & 10 \\
flugpl & 16 & 28 & 9 & 1 & $00: 06$ & 15 & 15 \\
bm23 & 20 & 190 & 11 & 30 & $06: 31$ & 11 & 11 \\
enigma & 21 & 118 & 12 & 183 & $44: 02$ & 12 & 12 \\
air01 & 23 & 137 & 13 & 3 & $01: 03$ & 21 & 21 \\
rgn & 24 & 75 & 13 & 6 & $60: 00$ & - & 21.82 \\
\hline
\end{tabular}

Table 7.1: Computational results of formulation (DG1).

\begin{tabular}{|l|rrr|rrrr|}
\hline Name & $|V|$ & $|E|$ & $c$ & \multicolumn{2}{c}{ (DG2) } & \multicolumn{2}{c|}{$\begin{array}{c}\text { Incumbent } \\
\end{array}$} \\
\hline \hline mod008 & 6 & 15 & 4 & 26 & $00: 03$ & 4 & 4 \\
stein9 & 13 & 66 & 7 & 671 & $46: 21^{*}$ & 7 & 8.73 \\
p0040 & 13 & 30 & 7 & 889 & $31: 16^{*}$ & 10 & 12.75 \\
p0033 & 15 & 40 & 8 & 429 & $23: 12^{*}$ & 11 & 14.57 \\
gt1 & 15 & 61 & 8 & 396 & $37: 45^{*}$ & 9 & 13.63 \\
flugpl & 16 & 28 & 9 & 230 & $11: 48$ & 15 & 15 \\
bm23 & 20 & 190 & 11 & 1 & $02: 53^{*}$ & - & 20.00 \\
enigma & 21 & 118 & 12 & 16 & $40: 41^{*}$ & 12 & 21.00 \\
air01 & 23 & 137 & 13 & 2 & $07: 42^{*}$ & - & 23.00 \\
rgn & 24 & 75 & 13 & 22 & $60: 00$ & 19 & 24.00 \\
\hline
\end{tabular}

Table 7.2: Computational results of formulation (DG2). 
Thus, when choosing between (DG1) and (DG2) the results in Table 7.1 and 7.2 clearly suggest to take (DG1). Of course, for either of these formulations it is true that their performance can be improved by using knowledge of the polyhedral structure within a branch-and-cut context as is done in the next subsection.

\subsubsection{Branch-and-cut}

We implemented a branch-and-cut algorithm which uses the results described in Section 7.3. The structure of this algorithm is depicted in Figure 7.4. The linear programs were solved with CPLEX 2.1 , the remainder of the algorithm, including branching, was implemented in $\mathrm{C}$ on a Silicon Graphics Indigo workstation with $32 \mathrm{Mb}$ of main memory.

Our algorithm does not differ too much from standard branch-and-cut algorithms, except that at several points we could benefit from specific knowledge of the problem instances we wanted to solve. The objective for all instances, consisting of a graph $G=(V, E)$ and an integer $c \geq 2$, is to maximize the number of vertices of $G$ that can be assigned to some component such that no component has more than $c$ vertices. If $i, j \in V, i \neq j$ are such that $N(i) \subseteq N(j)$ and there exists an optimal vertex assignment in which vertex $j$ is assigned to some component, then there also exists an optimal vertex assignment in which vertex $i$ is assigned to some component. The same holds if $\{i, j\} \in E$ and $N(i) \backslash\{j\} \subseteq N(j) \backslash\{i\}$. This implies that, for $i, j \in V, i \neq j$ with $N(i) \backslash\{j\} \subseteq N(j) \backslash\{i\}$, we can add the inequality $y_{i} \geq y_{j}$ to our linear program, although it is not valid for $P(G, c)$, but at least one optimal integral solution is not cut off by this inequality. If $N(i) \backslash\{j\}=N(j) \backslash\{i\}$ we add only one of the inequalities $y_{i} \leq y_{j}$ and $y_{i} \geq y_{j}$, since otherwise all optimal solutions could be cut off, namely if it is optimal to assign exactly one of the vertices $i$ and $j$. Constraints of this type are added to the linear program in the preprocessing phase of our algorithm, and they are never deleted later on.

Our initial linear program consists of the capacity constraints and the connectivity constraints. The 'cutting' part of our branch-and-cut algorithm uses the following classes of (facet defining) inequalities.

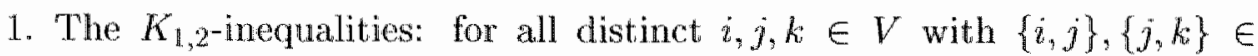
$E$ the inequality $y_{i}+y_{j}+y_{k}-x_{i k} \leq 2$ must be satisfied. Searching 


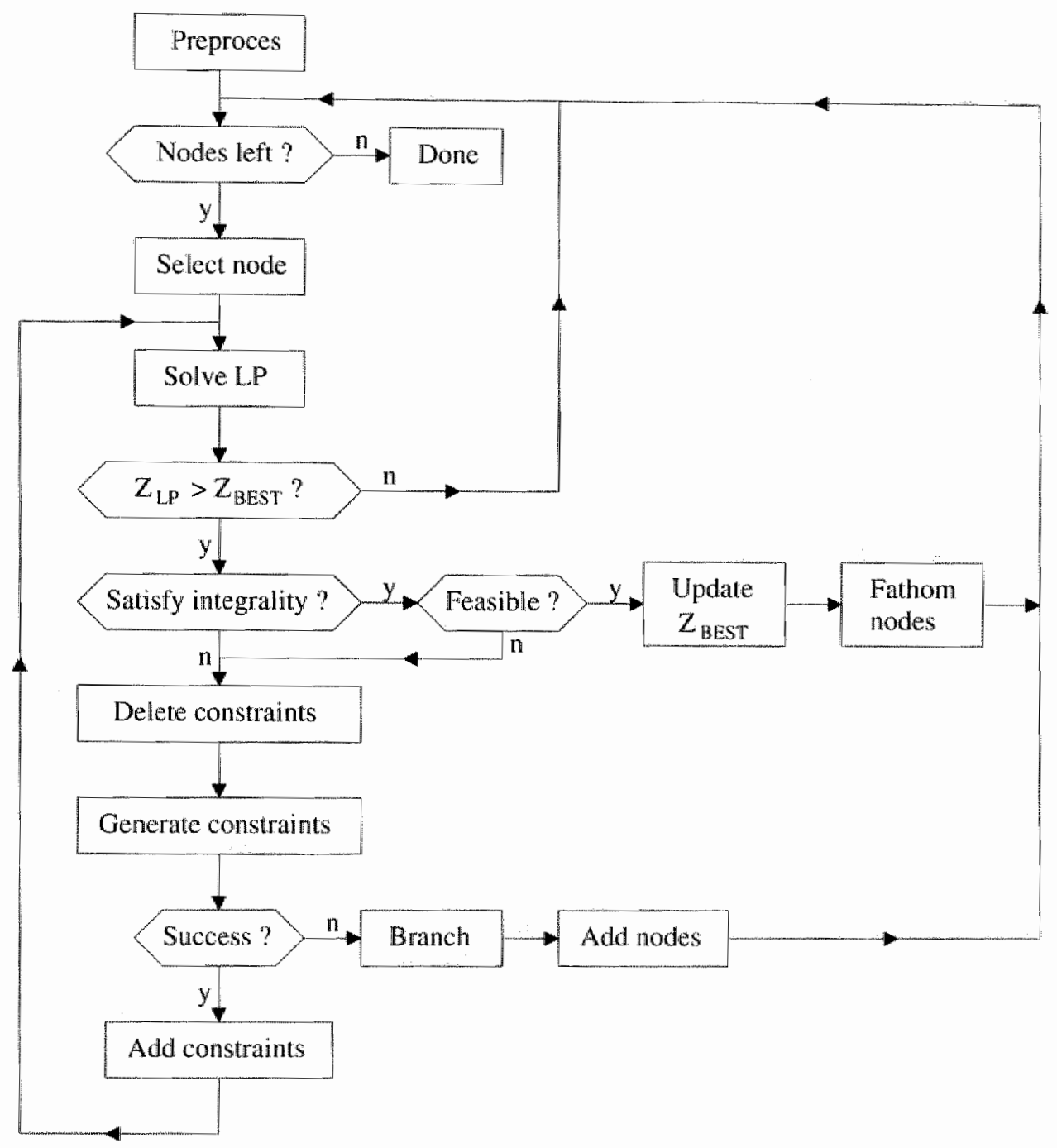

Figure 7.4: Structure of the branch-and-cut algorithm. 
violated $K_{1,2}$-inequalities is done by enumeration. The number of $K_{1,2}$ inequalities is $O\left(n^{3}\right)$ and enumeration can be done fast.

2. The cover inequalities (7.13) with $|W|=c+k$. We use the following heuristic to separate cover inequalities with $k=1$ and $k=2$ (compare Borndörfer et al. [9])

For each edge $\{i, j\} \in E$

Begin

Set $W:=\{i, j\}$ and $N:=N(i) \cap N(j)$

While $(N \neq \varnothing)$ and $(|W|<c+2)$ do

Begin

Choose $l \in N$

$W:=W \cup\{l\}$

$N:=\{v \in V \backslash W:|E(\{v\}, W)| \geq 2\}$

End

If $|W|=c+2 \rightarrow$ Check cover inequality

End

In the worst case this takes $O\left(n^{3} c^{2}\right)$ time.

3. The star inequalities (7.15). The number of star inequalities is linear in $n$, and therefore separation for this class is simply done by enumeration.

4. The 4-cycle inequalities (7.20). Searching violated inequalities in this class is done by enumeration, which takes $O\left(n^{4}\right)$ in the worst case.

5. The clique inequalities (7.14), constraints (7.16), and constraints (7.17). Finding violated inequalities in all three classes requires the search for a (maximal) clique in $G$. Maximal cliques in $G$ can be constructed via a recursive algorithm as follows. We assume that there is an order $\left(v_{1}, \ldots, v_{n}\right)$ of the vertices in $V$. Call the subroutine Clique with $U=\varnothing$ and $N=V$, where Clique has the following structure.

Clique $(U, N)$

Begin

$k:=\min \left\{l \mid\left(v_{l} \in N\right) \wedge\left(l>\max \left\{m \mid v_{m} \in U\right\}\right)\right\}$

While $(k \leq n)$ do

Begin

$U:=U \cup\left\{v_{k}\right\}$

$N^{\prime}:=\left\{v \in N \mid\left\{v, v_{k}\right\} \in E\right\}$

if $\left(N^{\prime}=\varnothing\right) \rightarrow U$ is maximal clique 


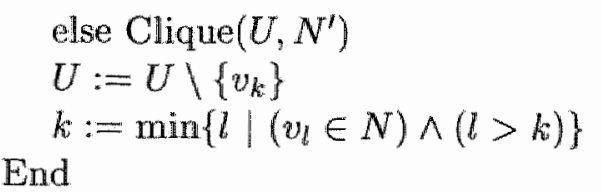

End

Notice that Clique is an exact algorithm for finding all maximal cliques in a graph and hence has exponential running time in the worst case. However, it is very fast in practise, cf. Carraghan and Pardalos [12]. Whenever a (maximal) clique inducing subset $U \subseteq V$ has been constructed the corresponding inequalities (7.14), (7.16) and (7.17) are checked. Violated inequalities are added to the current linear program.

6. The arrow constraints (7.19). Searching violated arrow inequalities is done by enumeration, which takes $O\left(n^{4}\right)$ in the worst case.

7. The triangle inequalities $x_{i j}+x_{i k}-x_{j k} \leq 1$ for all distinct $i, j, k \in V$. Separation is done by enumeration.

Each class of cutting planes is searched only if the number of violated inequalities found in all the previous classes is less then a given number. The class of triangle inequalities is searched only if the current solutions satisfies all integrality conditions and no other violated inequalities are found.

The branching part of our algorithm uses a depth-first search. We branch on the variable $y_{i}(i \in V)$ for which $\min \left\{y_{i}, 1-y_{i}\right\}$ is maximal. If $y_{i}<0.5$ we first examine the branch corresponding to $y_{i}=0$, if $y_{i} \geq 0.5$ we start with the case where $y_{i}=1$.

In order to be able to compare our computational results with Borndörfer et al. [10] we used again instances from the MIPLIB and NETLIB problem libraries.

We restricted ourselves to those instances with at most 100 vertices. Further, we used the same value for the capacity, i.e., $c=\left\lceil\frac{1.05 .1 \mathrm{~V}}{2}\right\rceil$ for the MIPLiB instances, and $c=\left\lceil\frac{1}{4}|V|\right\rceil$ for the NeturB instances. In order to avoid difficulties arising when comparing different algorithms ran on different computer platforms we took the number of nodes in the branching tree as performance measure. Each instance was allowed 24 hours of computing time. The performance of our branch-and-cut algorithm on these instances is given in Tables 7.3 and 7.4. Both tables have the same structure. The first column lists the name of each problem, and the next three columns give the size of the corresponding 
graph (number of vertices, and number of edges) and the capacity. The column labeled 'Nod. $\mathbb{B}$ ' denotes the number of nodes (of the branch-and-bound tree) Borndörfer et al. needed to solve their problem. The next two columns list the number of nodes and iterations we needed (an iteration of our algorithm consists of one round of adding violated inequalities and reoptimizing the linear program). The last two columns give the used lower bound and the optimal value if the instance was solved within 24 hours. Finally, when an asterisk is attached to an instance, it means that the branch-and-cut algorithm described here did not solve the corresponding instance to optimality within 24 hours of computing time. In that case, the last column gives the value of the upper bound found (which is not necessarily equal to the value of the optimal solution).

It is important to stress here that Borndörfer et al. [10] take $B=2$ for all instances of (DG2) arising from the MIPLIB problem library, and $B=4$ for all instances taken form the NETLIB problem library. That is, they require a partition of the assigned vertices into at most 2 and 4 components, respectively. In our case, we allow for an arbitrary number of components, thus we solve a different, more general problem. Obviously, it follows that the value of an optimal solution we find may differ from the one found by Borndörfer et al. [10]. In fact, since we use the optimal value found in [10] as a lower bound for our problem instances, comparing columns 'Lb.' and 'Obj.' in Tables 7.3 and 7.4 indicates when allowing more than 2 (4) components is beneficial.

The results in Tables 7.3 and 7.4 show that a branch-and-cut approach based upon formulation (DGI) is a viable approach to solving instances of DG. Out of the 46 instances considered here, our algorithm needed 24 times less nodes than the algorithm in [10] for solving a more general problem, whereas their algorithm is superior in 11 cases. Ten problems were solved in the root node with both algorithms, and 1 problem (misc03) was solved with neither of the algorithms. Finally, in 6 out of the 46 cases allowing an arbitrary number of components yields a better solution (notice 'stein15'!).

\subsubsection{The influence of varying capacity}

Another issue we investigated is the influence of the capacity on the number of components in an optimal solution and the hardness of the problem. To do this, we fixed four different values for the capacity and ran each of the fourteen 


\begin{tabular}{|l|rrr|r|rr|rr|}
\hline Name & $|V|$ & $|E|$ & $c$ & Nod.B & Nodes & Iter. & Lb. & Obj. \\
\hline \hline mod008 & 6 & 15 & 4 & 1 & 1 & 1 & 4 & 4 \\
stein9 & 13 & 66 & 7 & 16 & 5 & 7 & 7 & 7 \\
p0040 & 13 & 30 & 7 & 1 & 1 & 5 & 10 & 10 \\
p0033 & 15 & 40 & 8 & 1 & 1 & 2 & 12 & 12 \\
gt1 & 15 & 61 & 8 & 13 & 3 & 27 & 10 & 10 \\
flugpl & 16 & 28 & 9 & 1 & 1 & 10 & 14 & 15 \\
bm23 & 20 & 190 & 11 & 1 & 1 & 2 & 11 & 11 \\
enigma & 21 & 118 & 12 & 28 & 5 & 27 & 12 & 12 \\
air01 & 23 & 137 & 13 & 1 & 3 & 12 & 20 & 21 \\
rgn & 24 & 75 & 13 & 13 & 5 & 98 & 19 & 19 \\
pipex & 25 & 153 & 14 & 13 & 7 & 79 & 16 & 16 \\
lseu & 28 & 136 & 15 & 1 & 7 & 57 & 21 & 21 \\
gt2 & 28 & 173 & 15 & 28 & 9 & 21 & 17 & 17 \\
sentoy & 30 & 435 & 16 & 1 & 1 & 1 & 16 & 16 \\
stein15 & 36 & 350 & 19 & 5458 & 13 & 19 & 19 & 29 \\
misc02 & 43 & 454 & 23 & 34 & 59 & 1021 & 28 & 29 \\
sample2 & 45 & 97 & 24 & 7 & 39 & 2297 & 41 & 41 \\
air02 & 50 & 1126 & 27 & 1 & 9 & 12 & 28 & 28 \\
misc01 & 54 & 929 & 29 & 367 & 171 & 972 & 30 & 31 \\
mod013 & 62 & 144 & 33 & 13 & 7 & 393 & 56 & 56 \\
mod014 & 74 & 127 & 39 & 1 & 1 & 4 & 72 & 72 \\
Ip41 & 85 & 1644 & 45 & 1129 & 21 & 223 & 50 & 50 \\
bell5 & 87 & 226 & 46 & 13 & 9 & 23 & 83 & 83 \\
p0291 & 92 & 521 & 49 & 19 & 13 & 80 & 85 & 85 \\
misc03* & 96 & 2894 & 51 & 12745 & 1 & 194 & 52 & 72 \\
1152lav* & 97 & 1866 & 51 & 1525 & 1 & 173 & 61 & 78 \\
khb05250* & 100 & 1323 & 53 & 73 & 1 & 162 & 75 & 87 \\
harp2 & 100 & 1225 & 53 & 1 & 15 & 119 & 83 & 83 \\
\hline
\end{tabular}

Table 7.3: Computational results of the MrPLIB instances. 


\begin{tabular}{|l|rrr|r|rr|rr|}
\hline Name & $|V|$ & $|E|$ & $c$ & Nod.B & Nodes & Iter. & Lb. & Obj. \\
\hline \hline seba & 2 & 0 & 1 & 1 & 1 & 1 & 2 & 2 \\
afiro & 20 & 20 & 5 & 1 & 1 & 2 & 18 & 18 \\
fit1d & 24 & 228 & 6 & 6 & 9 & 72 & 8 & 8 \\
fit2d & 25 & 279 & 7 & 41 & 25 & 177 & 7 & 7 \\
sc50b & 28 & 110 & 7 & 56 & 11 & 103 & 17 & 17 \\
sc50a & 29 & 95 & 8 & 26 & 3 & 18 & 21 & 21 \\
kb2 & 39 & 330 & 10 & 1 & 9 & 66 & 25 & 25 \\
vtpbase & 51 & 354 & 13 & 561 & 11 & 190 & 37 & 37 \\
bore3d & 52 & 615 & 13 & 51 & 43 & 78 & 29 & 29 \\
adlittle & 53 & 239 & 14 & 11 & 1 & 14 & 43 & 43 \\
blend & 54 & 548 & 14 & 61 & 21 & 491 & 34 & 34 \\
recipe & 55 & 129 & 14 & 1 & 1 & 3 & 54 & 55 \\
scagr7 & 58 & 661 & 15 & 16 & 29 & 47 & 37 & 37 \\
sc105 & 59 & 356 & 15 & 66 & 17 & 345 & 43 & 43 \\
stocfor1 & 62 & 272 & 16 & 21 & 3 & 337 & 52 & 52 \\
scsd1 & 77 & 202 & 20 & 21 & 1 & 8 & 69 & 69 \\
beaconfd & 90 & 1199 & 23 & 121 & 117 & 252 & 64 & 64 \\
share2b & 93 & 619 & 24 & 626 & 1 & 6 & 84 & 84 \\
\hline
\end{tabular}

Table 7.4: Computational results of the NETLIB instances.

smallest MIPLiB instances. The results can be found in Table 7.5, where the column labeled \#C indicates the number of components found. Notice that the value of the lower bound mentioned in these tables is found using the value of an optimal solution to an instance with a smaller capacity. To be precise, with $\left(c_{0}, \ldots, c_{4}\right)=(0,3,5,10,15)$, let opt $\left(c_{i}\right)$ equal the optimal value of an instance with capacity $c_{i}$ and let $\mathrm{Lb}_{i}$ denote the lower bound used in an instance with capacity $c_{i}(i=1, \ldots, 4)$. We have: $\mathrm{Lb}_{i}=\min \left\{\max \left\{c_{i}, \operatorname{opt}\left(c_{i-1}\right)\right\}, n\right\}$ for $i=1, \ldots, 4$, and opt $\left(c_{0}\right)=0$. From the results in Table 7.5 it seems difficult to make a general statement regarding a relation between the capacity and the hardness of the problem. However, the instances enigma, rgn, lseu, and gt 2 seem to suggest that the problems tend to get more difficult with increasing capacity. 


\begin{tabular}{|l|rrr|r|rr|}
\hline Name & $c$ & Lb. & Obj. & \#C & Nodes & Iter. \\
\hline \hline mod008 & 3 & 3 & 3 & 1 & 1 & 2 \\
$|V|=6$ & 5 & 5 & 5 & 1 & 1 & 1 \\
$|E|=15$ & 10 & 6 & 6 & 1 & 1 & 1 \\
& 15 & 6 & 6 & 1 & 1 & 1 \\
\hline stein9 & 3 & 3 & 3 & 1 & 15 & 39 \\
$|V|=13$ & 5 & 5 & 5 & 1 & 13 & 35 \\
$|E|=66$ & 10 & 10 & 10 & 1 & 1 & 3 \\
& 15 & 13 & 13 & 1 & 1 & 1 \\
\hline p0040 & 3 & 3 & 10 & 10 & 1 & 7 \\
$|V|=13$ & 5 & 10 & 10 & 10 & 1 & 3 \\
$|E|=30$ & 10 & 10 & 10 & 10 & 3 & 8 \\
& 15 & 13 & 13 & 1 & 1 & 1 \\
\hline p0033 & 3 & 3 & 9 & 6 & 9 & 33 \\
$|V|=15$ & 5 & 9 & 10 & 5 & 6 & 23 \\
$|E|=40$ & 10 & 10 & 13 & 3 & 23 & 77 \\
& 15 & 15 & 15 & 1 & 1 & 1 \\
\hline gt1 & 3 & 3 & 9 & 9 & 2 & 18 \\
$|V|=15$ & 5 & 9 & 9 & 9 & 3 & 9 \\
$|E|=61$ & 10 & 10 & 11 & 2 & 3 & 9 \\
& 15 & 15 & 15 & 1 & 1 & 1 \\
\hline flugpl & 3 & 3 & 11 & 6 & 6 & 29 \\
$|V|=16$ & 5 & 11 & 14 & 3 & 1 & 4 \\
$|E|=28$ & 10 & 14 & 15 & 2 & 2 & 9 \\
& 15 & 15 & 15 & 1 & 1 & 3 \\
\hline bm23 & 3 & 3 & 3 & 1 & 1 & 3 \\
$|V|=20$ & 5 & 5 & 5 & 1 & 1 & 3 \\
$|E|=190$ & 10 & 10 & 10 & 1 & 1 & 3 \\
& 15 & 15 & 15 & 1 & 1 & 2 \\
\hline
\end{tabular}

Table 7.5: Computational results for varying capacity. 


\begin{tabular}{|l|rrr|r|rr|}
\hline Name & $c$ & Lb. & Obj. & \#C & Nodes & Iter. \\
\hline \hline enigma & 3 & 3 & 10 & 10 & 3 & 26 \\
$|V|=21$ & 5 & 10 & 10 & 10 & 3 & 17 \\
$|E|=118$ & 10 & 10 & 11 & 2 & 7 & 34 \\
& 15 & 15 & 15 & 1 & 11 & 35 \\
\hline air01 & 3 & 3 & 6 & 2 & 5 & 18 \\
$|V|=23$ & 5 & 6 & 10 & 2 & 8 & 25 \\
$|E|=137$ & 10 & 10 & 18 & 2 & 7 & 29 \\
& 15 & 18 & 22 & 2 & 1 & 12 \\
\hline rgn & 3 & 3 & 12 & 4 & 11 & 37 \\
$|V|=24$ & 5 & 12 & 14 & 3 & 74 & 284 \\
$|E|=75$ & 10 & 14 & 19 & 2 & 10 & 79 \\
& 15 & 19 & 19 & 2 & 120 & 898 \\
\hline pipex & 3 & 3 & 16 & 16 & 1 & 17 \\
$|V|=25$ & 5 & 16 & 16 & 16 & 1 & 7 \\
$|E|=153$ & 10 & 16 & 16 & 16 & 3 & 12 \\
& 15 & 16 & 16 & 16 & 7 & 33 \\
\hline lseu & 3 & 3 & 18 & 16 & 1 & 13 \\
$|V|=28$ & 5 & 18 & 18 & 16 & 5 & 36 \\
$|E|=136$ & 10 & 18 & 20 & 11 & 10 & 67 \\
& 15 & 20 & 21 & 6 & 15 & 129 \\
\hline gt2 & 3 & 3 & 17 & 17 & 1 & 13 \\
$|V|=28$ & 5 & 5 & 17 & 17 & 1 & 4 \\
$|E|=173$ & 10 & 10 & 17 & 17 & 3 & 24 \\
& 15 & 17 & 17 & 17 & 11 & 73 \\
\hline sentoy & 3 & 3 & 3 & 1 & 1 & 3 \\
$|V|=30$ & 5 & 5 & 5 & 1 & 1 & 3 \\
$|E|=435$ & 10 & 10 & 10 & 1 & 1 & 3 \\
& 15 & 15 & 15 & 1 & 1 & 3 \\
\hline
\end{tabular}

Table 7.5: Computational results for varying capacity (continued). 


\subsection{Conclusions}

In this chapter, we gave two formulations for the problem of disconnecting graphs by removing vertices. It turns out that from a theoretical point of view as well as from a computational point of view (DG1) is more suited to formulate this problem then (DG2). When dealing with problems where a nontrivial upper bound on the number of components to be formed is known a priori, (DG2) could be more appropriate.

Further, we studied the facial structure of the polytope associated to (DG1). This is mainly done by establishing ties with other polytopes, notably the (capacitated) clique partitioning polytope. It turns out that a number of facet defining inequalities for this polytope remain facet defining for the polytope associated to (DG1). We characterized all facet defining inequalities with right-hand side 1 .

Finally, we did limited computational experiments by implementing a branchand-cut algorithm using the polyhedral results we derived. Although it is tempting to compare the results presented here with the results of Borndörfer et al. $[9,10]$ for (DG2), one should be careful in doing so, since in the latter papers a different problem is solved due to restricting the number of components to be found.

From our computational results we conclude that (DG1) is a useful model at least for modest problem instances. Using a relatively simple code (it is made up of only 1250 lines), we could solve most instances with 100 vertices or less by branch-and-cut without evaluating more than 100 nodes or 2500 iterations. More knowledge of the polyhedral structure of $P(G, c)$ will probably make it possible to solve larger problem instances.

\section{Acknowledgements}

This chapter is based on joint work with Maarten Oosten and Frits Spieksma. 
$\mathrm{B}$

a 


\section{Chapter 8}

\section{Patching}

\subsection{Introduction}

The solutions to mathematical programming problems usually consist of subsets of a finite ground set, for instance all boolean vectors satisfying a given proposition, or all independent sets or Hamiltonian cycles in a graph. Such solutions can easily be represented as binary vectors, so that $X \subseteq\{0,1\}^{n}$ is the set of (incidence vectors of) solutions. Optimizing some function over the poly tope $P(X):=\operatorname{conv}(X)$ requires at least a partial description of $P(X)$, and the more faces and facets of this polytope are known, the larger the probability that one can solve the corresponding mathematical programming problem. In this chapter we describe two variants of patching, a technique to construct facets from facets, which can be applied to arbitrary $\{0,1\}$-polytopes that contain the origin and all unit vectors. The first variant is called intersection patching, and it generalizes the patching technique for the clique partitioning polytope, as described in Chapter 2 . The second variant is called disjoint patching. This is not a generalization of the procedures described in Chapter 3 , but rather a mixture of sequential and simultaneous lifting. We illustrate our results with the independent set polytope, the monotone traveling salesman polytope, and the knapsack polytope. 


\subsection{Intersection patching}

Before describing intersection patching we make some assumptions that hold throughout the remainder of this chapter. $I=\{1, \ldots, n\}$ is an index set, and $X \subseteq\{0,1\}^{n}$ is a subset of the unit hypercube in $\mathbb{R}^{n}$ containing the origin and all unit vectors. The polytope $P(X)$ is defined as the convex hull of all vectors in $X$. It is not hard to see that the inequalities $x_{i} \geq 0(i \in I)$ define facets of $P(X)$. In fact, these inequalities are the only facet inducing inequalities with right-hand side 0 . For all $I^{\prime} \subseteq I$ we define

$$
\begin{aligned}
& L\left(I^{\prime}\right):=\left\{x \in X \mid x_{i}=0 \quad \forall i \notin I^{\prime}\right\} \\
& R\left(I^{\prime}\right):=\left\{y \in X \mid x+y \in X \quad \forall x \in L\left(I^{\prime}\right)\right\}
\end{aligned}
$$

In other words, $L\left(I^{\prime}\right)$ is the set of points in $X$ that have zero entries outside $I^{\prime}$, and $R\left(I^{\prime}\right)$ is the set of points in $X$ that can be added to any point in $L\left(I^{\prime}\right)$ without leaving $X$. Notice that both $L\left(I^{\prime}\right)$ and $R\left(I^{\prime}\right)$ are nonempty, because both sets contain the zero vector. Moreover, $L\left(I^{\prime}\right)$ contains $\left|I^{\prime}\right|$ unit vectors, and this implies that all elements of $R\left(I^{\prime}\right)$ have zero entries on $I^{\prime}$. The next definition gives the load and the residual of a subset of $I$ with respect to a valid inequality for $P(X)$,

Definition 8.1 Let $a^{T} x \leq a_{0}$ be a valid inequality for $P(X)$, and let $I^{\prime} \subseteq I$. The load and the residual of $I^{\prime}$ with respect to $a^{T} x \leq a_{0}$ are given by

$$
\begin{aligned}
\operatorname{load}\left(a, I^{\prime}\right) & :=\max \left\{a^{T} x \mid x \in L\left(I^{\prime}\right)\right\} \\
\operatorname{res}\left(a, I^{\prime}\right) & :=\max \left\{a^{T} x \mid x \in R\left(I^{\prime}\right)\right\}
\end{aligned}
$$

Notice that the load and the residual are always nonnegative, since both $L\left(I^{\prime}\right)$ and $R\left(I^{\prime}\right)$ contain the zero vector. For all $x \in L\left(I^{\prime}\right)$ and $y \in R\left(I^{\prime}\right)$ we have that $x+y \in X$, hence

$$
\max _{x \in L\left(I^{\prime}\right)} a^{T} x+\max _{y \in R\left(I^{\prime}\right)} a^{T} y \leq \max _{z \in X} a^{T} z \leq a_{0}
$$

since $a^{T} x \leq a_{0}$ is assumed to be valid for $P(X)$. The intersection patching technique uses the following definition. 
Definition 8.2 Let $a^{T} x \leq a_{0}$ be a valid inequality for $P(X)$, and let $I_{1}, \ldots, I_{k}$ be subsets of $I$. The intersection graph $G\left(I_{1}, \ldots, I_{k} ; a\right)=(U, F)$ is defined by $U=\{1, \ldots, k\}$, and $\{i, j\} \in F$ if and only if there is an $r \in I_{i} \cap I_{j}$ with $a_{r} \neq 0$.

Now we have all ingredients for describing intersection patching.

Theorem 8.3 (Intersection patching)

Let $a^{T} x \leq a_{0}$ be a valid inequality for $P(X)$, and let $I_{1}, \ldots, I_{k} \subseteq I$ be such that

(i) $\bigcup_{l=1}^{k} I_{l}=I$,

(ii) $\operatorname{load}\left(a, I_{l}\right)>0$ and $\sum_{i \in I_{l}} a_{l} x_{i} \leq \operatorname{load}\left(a, I_{l}\right)$ defines a facet of $P\left(L\left(I_{l}\right)\right)$ for each $l=1, \ldots, k$,

(iii) $\operatorname{load}\left(a, I_{l}\right)+\operatorname{res}\left(a, I_{l}\right)=a_{0}$ for all $l=1, \ldots, k$,

(iv) The intersection graph $G\left(I_{1}, \ldots, I_{k} ; a\right)$ is connected.

Then the inequality $a^{T} x \leq a_{0}$ defines a facet of $P(X)$.

Proof The face $F_{a}:=\left\{x \in P(X) \mid a^{T} x=a_{0}\right\}$ is contained in the facet $F_{d}$ of $P(X)$ defined by some valid inequality $d^{T} x \leq d_{0}$. First, we show that there exists a $\beta_{l}>0$ such that $d_{i}=\beta_{l} \cdot a_{i}$ for all $i \in I_{l}(l=1, \ldots, k)$. For each $l=1, \ldots, k$ choose a maximal set $M_{l}$ of affinely independent integral points $x \in P\left(L\left(I_{l}\right)\right)$ satisfying $a^{T} x=\operatorname{load}\left(a, I_{l}\right)$. According to condition (iii) we can find a vector $y^{l} \in R\left(I_{l}\right)$ with $a^{T} y^{l}=\operatorname{res}\left(a, I_{l}\right)$ and $x+y^{l} \in P\left(L\left(I_{l}\right)\right)$ for all $x \in M_{l}$, thus yielding

$$
\begin{aligned}
a^{T}\left(x+y^{l}\right) & =a^{T} x+a^{T} y^{l} \\
& =\operatorname{load}\left(a, I_{l}\right)+\operatorname{res}\left(a, I_{l}\right) \\
& =a_{0} \quad \forall x \in M_{l} \quad(l=1, \ldots, k)
\end{aligned}
$$

Since none of the facets described in condition (ii) contains the zero vector, each set $M_{l}$ is linearly independent, and hence $\left\{x+y^{l} \mid x \in M_{l}\right\}$ is also linearly 
independent. It follows that for each $l=1, \ldots, k$ there exists a $\beta_{l}>0$ such that $d_{i}=\beta_{l} \cdot a_{i}$ for all $i \in I_{l}$.

It remains to show that $\beta_{l}=\beta_{m}$ for all $l, m=1, \ldots, k$. Let $l, m \in\{1, \ldots, k\}$ be such that the corresponding vertices in the intersection graph $G\left(I_{1}, \ldots, I_{k} ; a\right)$ are adjacent. Then there exists $i \in I_{l} \cap I_{m}$ with $a_{i} \neq 0$ and $\beta_{l} \cdot a_{i}=\beta_{m} \cdot a_{i}$, hence $\beta_{l}=\beta_{m}$. Since the intersection graph is connected it follows that $\beta_{1}=\ldots=\beta_{k}$.

Notice that Theorem 8.3 generalizes the patching procedure for the clique partitioning polytope given in Theorem 2.13: if $X \subseteq\{0,1\}^{n}$ is the set of incidence vectors of all clique partitions of $G=(V, E)$ with $|E|=n$, then $X$ contains the zero vector and all unit vectors, and Theorem 8.3 can be applied to construct facets of the clique partitioning polytope $P(G)$. Here we show that patching is well suited for constructing facets of other polytopes as well.

Let $G=(V, E)$ be a graph, then a subset $U \subseteq V$ is called an independent set if $E(U)=\varnothing$. The independent set polytope $P_{I S}(G) \subseteq \mathbb{R}^{|V|}$ is the convex hull of all incidence vectors of independent sets of $G$. This polytope is the subject of research of e.g. Chvátal [18] and Padberg [60]. Obviously, $P_{I S}(G)$ contains the origin and all unit vectors. Moreover, if $U \subseteq V$ is an independent set, then all subsets of $U$ are also independent sets. Before we can apply Theorem 8.3 to the independent set polytope we need to know at least something of its structure. If $U \subseteq V$ and $(U, E(U))$ is a clique in $G$, then no independent set contains more than one vertex from $U$, hence the clique inequality

$$
\sum_{i \in U} x_{i} \leq 1
$$

is valid for $P_{I S}(G)$. Padberg [60] showed that these inequalities define facets if and only if $U$ induces a maximal clique in $G$. Another class of facets is associated with odd holes. A subgraph $(U, E(U))$ of $G$ is called a hole if it is a chordless cycle. Obviously, if $(U, E(U))$ is a hole in $G$, then no independent set contains more than $\left\lfloor\frac{1}{2}|U|\right\rfloor$ vertices from $U$, hence the hole inequality

$$
\sum_{i \in U} x_{i} \leq\left\lfloor\frac{1}{2}|U|\right\rfloor
$$

is valid for $P_{I S}(G)$. If $|U|$ is even (8.2) is nothing but the sum of $\frac{1}{2}|U|$ clique inequalities, and therefore it is not facet defining. However, if $|U|$ is odd 


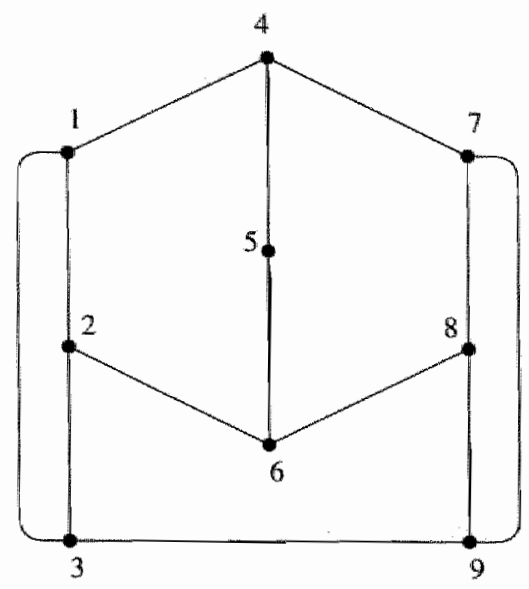

Figure 8.1: An instance of the independent set problem.

and for each vertex $v \in V \backslash U$ there exists an independent set $U^{x} \subseteq U$ with $\left|U^{\prime}\right|=\left\lfloor\frac{1}{2}|U|\right\rfloor$ such that $\{v\} \cup U^{\prime}$ is an independent set, then the corresponding odd hole inequality (8.2) defines a facet of $P_{I S}(G)$, see Padberg [60].

The facets defined by (8.1) and (8.2) form our building blocks for illustrating Theorem 8.3. Consider the graph $G=(V, E)$ of Figure 8.1. It is not hard to check that the inequality

$$
\sum_{i=1}^{9} x_{i} \leq 3
$$

is valid for $P_{I S}(G)$. Denote (8.3) by $a^{T} x \leq a_{0}$, and define $I_{1}, \ldots, I_{4} \subseteq V$ as follows.

$$
\begin{array}{ll}
I_{1}:=\{1,2,3\} & I_{3}:=\{1,2,4,5,6\} \\
I_{2}:=\{7,8,9\} & I_{4}:=\{4,5,6,7,8\}
\end{array}
$$

The subgraph induced by $I_{1}$ is a clique, hence $\operatorname{load}\left(a, I_{1}\right)=1$. Furthermore, every independent set in $I_{1}$ together with nodes 5 and 8 is an independent set of $G$, so res $\left(a, I_{1}\right)=2$. Symmetry shows that $\operatorname{load}\left(a, I_{2}\right)=1$ and $\operatorname{res}\left(a, I_{2}\right)=2$. The subgraph induced by $I_{3}$ is an odd hole of length 5 , 
hence load $\left(a, I_{3}\right)=2$. Since every independent set in $I_{3}$ together with node 9 is a independent set of $G$, we have res $\left(a, I_{3}\right)=1$. Analogously, we find that $\operatorname{load}\left(a, I_{4}\right)=2$ and $\operatorname{res}\left(a, I_{4}\right)=1$. The intersection graph $G\left(I_{1}, \ldots, I_{4} ; a\right)$ is the path $\{\{1,3\},\{3,4\},\{4,2\}\}$, which is connected. All conditions of Theorem 8,3 are fulfilled, and this shows that (8.3) defines a facet of $P_{I S}(G)$.

Two other examples of patching for the independent set polytope are found in Figure 3.2 on page 51. Let $G_{1}=\left(V_{1}, E_{1}\right)$ be the graph on the left, and let $G_{2}=\left(V_{2}, E_{2}\right)$ be the graph on the right. One easily verifies that $x\left(V_{1}\right) \leq 3$ is valid for $P_{I S}\left(G_{1}\right)$ and that $x\left(V_{2}\right) \leq 2$ is valid for $P_{I S}\left(G_{2}\right)$. Moreover, both inequalities define facets of the various polytopes. Indeed, the first inequality is obtained as a patching of two 5-cycles (both with load equal to 2 and residual equal to 1), and the second inequality is obtained as a patching of two cliques of size 3 (both with load and residual equal to 1) and a 5-cycle (with a load of 2 and a residual of 0 ). In both cases the intersection graph is a clique, so all conditions of Theorem 8.3 are met.

A second problem for which patching is a useful technique is the traveling salesman problem. Here one is given a complete graph $K_{n}=\left(V_{n}, E_{n}\right)$ together with nonnegative costs $c_{i j} \in \mathbb{R}_{+}$for all $\{i, j\} \in E_{n}$, and the problem is to find a Hamiltonian cycle in $K_{n}$ with minimal cost. The set $S \subseteq\{0,1\}^{\left|E_{n}\right|}$ of all incidence vectors of Hamiltonian cycles in $K_{n}$ contains neither the zero vector nor any of the unit vectors, so before using Theorem 8.3 we extend $S$ to

$$
X:=\left\{x \in\{0,1\}^{\left|E_{x}\right|} \mid \exists y \in S: x \leq y\right\}
$$

In other words, $X$ is the set of incidence vectors of all subsets of $E_{n}$ that can be extended to Hamiltonian cycles of $K_{n}$. We consider the polytope $\operatorname{TSP}\left(K_{n}\right):=\operatorname{conv}(X)$, the monotone symmetric traveling salesman poly tope. The (a)symmetric traveling salesman polytopes and their monotonizations have been studied intensively, see for instance Lawler et al. [49] for a general introduction, and see Grötschel and Padberg [34, 35], Grötschel and Pulleyblank [37], Naddef and Rinaldi [57], Balas and Fischetti [4] and Fischetti [28] for more detailed results. A very famous class of facets is formed by the comb inequalities introduced by Chvátal [17]. These inequalities can be described as follows. Let $k \geq 3$ be odd, and let $H, T_{1}, \ldots, T_{k} \subseteq V_{n}$ be such that the following holds.

(i) $\left|H \cap T_{i}\right|=1$ for all $i=1, \ldots, k$, 
(ii) $\left|T_{i} \backslash H\right| \geq 1$ for all $i=1, \ldots, k$,

(iii) $T_{i} \cap T_{j}=\varnothing$ for all $1 \leq i<j \leq k$.

The sets $H, T_{1}, \ldots, T_{k}$ together are called a comb; $H$ is called a handle, and $T_{1}, \ldots, T_{k}$ are called teeth. The comb inequality

$$
\sum_{e \in E_{n}(H)} x_{e}+\sum_{e \in E_{n}(T)} x_{e} \leq|H|+\sum_{i=1}^{k}\left(\left|T_{i}\right|-1\right)-\frac{k+1}{2}
$$

is valid and facet defining for $\operatorname{TSP}\left(K_{n}\right)$. Figure 8.2 gives an example of a comb with 3 teeth.

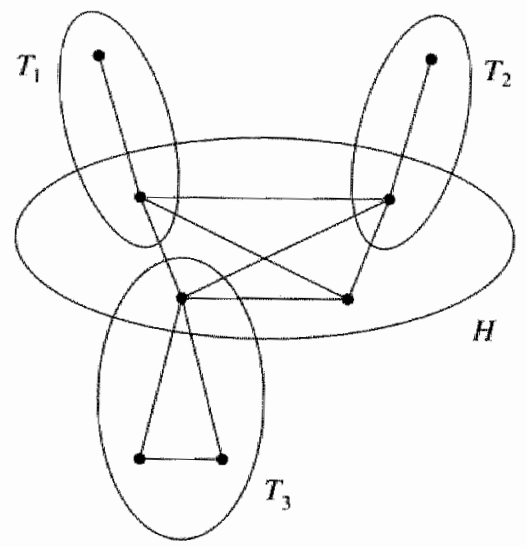

Figure 8.2; Support of a comb inequality.

We use the comb inequalities as building blocks to show that the so-called clique tree inequalities [37] define facets of the monotone symmetric traveling salesman polytope. The clique tree inequalities can be described as follows. Let $r \geq 1$ be an arbitrary integer and let $k \geq 3$ be odd. Let $H_{1}, \ldots, H_{r} \subseteq V_{n}$ and $T_{1}, \ldots, T_{k} \subseteq V_{n}$ be such that the following holds.

(i) $\left|H_{i} \cap T_{j}\right| \leq 1$ and $\sum_{j=1}^{k}\left|H_{i} \cap T_{j}\right|$ is odd for all $i=1, \ldots, r$ and $j=1, \ldots, k$,

(ii) $T_{j} \backslash\left(\bigcup_{i=1}^{r} H_{i}\right) \neq \varnothing$ for all $j=1, \ldots, k$,

(iii) $H_{i} \cap H_{j}=\varnothing$ for all $i, j=1, \ldots, r, i \neq j$,

(iv) $T_{i} \cap T_{j}=\varnothing$ for all $i, j=1, \ldots, k, i \neq j$, 
(v) The intersection graph $G\left(H_{1}, \ldots, H_{r}, T_{1}, \ldots, T_{k} ; 1\right)$ is a tree.

Then the clique tree inequality

$\sum_{i=1}^{r} \sum_{e \in E_{n}\left(H_{i}\right)} x_{e}+\sum_{i=1}^{k} \sum_{e \in E_{n}\left(T_{i}\right)} x_{e} \leq \sum_{i=1}^{r}\left|H_{i}\right|+\sum_{i=1}^{k}\left(\left|T_{i}\right|-t_{i}\right)-\frac{k+1}{2}$

(where $t_{i}$ is the number of handles met by $T_{i}$ ) is vallid for $\operatorname{TSP}\left(K_{n}\right)$. Figure 8.3 shows the smallest possible clique tree inequality with 3 handles.

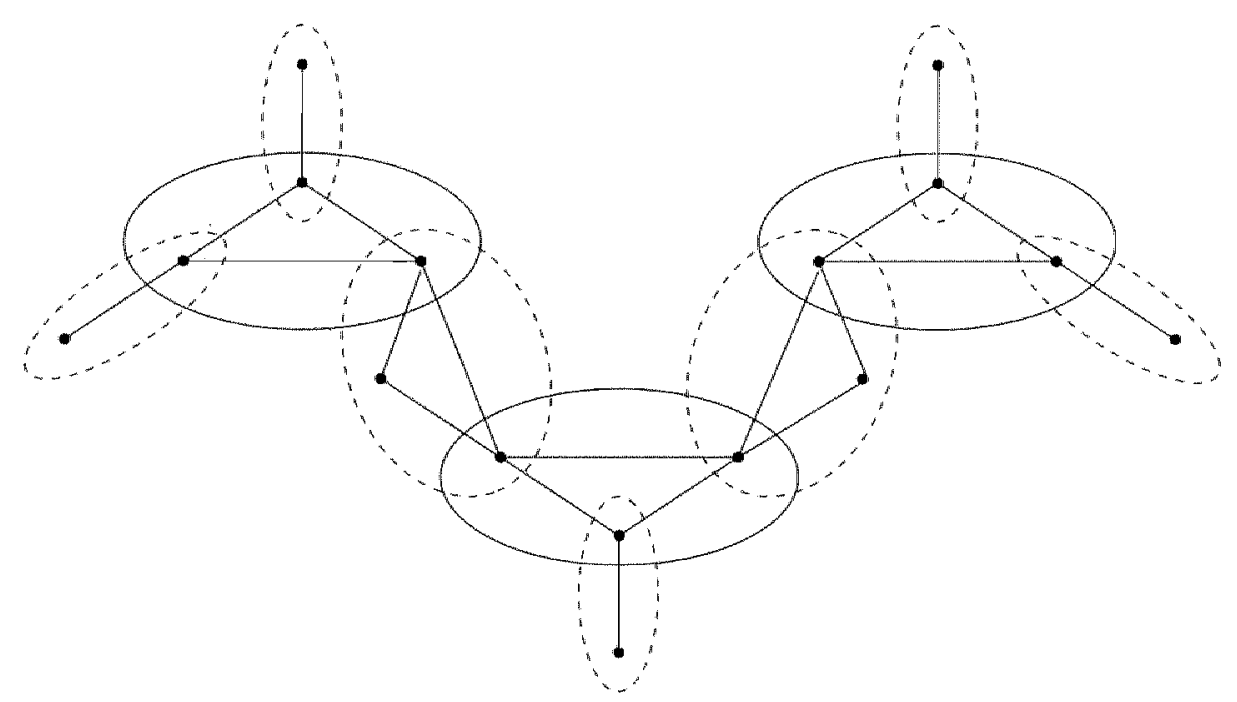

Figure 8.3: Support of a clique tree inequality. Solid ellipses indicate handles, dotted ellipses indicate teeth.

To prove that the clique tree inequalities (8.5) define facets of $T S P\left(K_{n}\right)$ we proceed as follows. Denote (8.5) by $a^{T} x \leq a_{0}$, and define $I_{j} \subseteq V_{n}$ as the union of the edge set of handle $H_{j}$ and the edge sets of all teeth that have a vertex in common with $H_{j}(j=1, \ldots, r)$, then $I_{j}$ induces a comb inequality. The load and the residual of $I_{j}(j=1, \ldots, r)$ with respect to the clique tree inequality add up to the right-hand side of (8.5). Furthermore, the intersection graph $G\left(I_{1}, \ldots, I_{r} ; a\right)$ is a tree. Conditions (ii)-(iv) of Theorem 8.3 hold, and 
this implies that the clique tree inequality defines a facet of $T S P(G)$, where $G=\left(V_{n}, E\right)$ and

$$
E:=\bigcup_{j=1}^{r} E_{n}\left(I_{j}\right)
$$

Since $\operatorname{TSP}\left(K_{n}\right)$ is a monotonization it is almost trivial to see that $(8.5)$ also defines a facet of this polytope. It boils down to identifying, for each edge $e^{*} \in E_{n} \backslash E$, a feasible set $A \subseteq E \cup\left\{e^{*}\right\}$ with $e^{*} \in A$ such that the incidence vector of $A$ satisfies (8.5) at equality. Hence, the clique tree inequalities define facets of $\operatorname{TSP}\left(K_{n}\right)$.

It should be pointed out here that the clique tree inequalities can be generalized in the following sense [37]: the restriction $\left|H_{i} \cap T_{j}\right| \leq 1$ for all $i=1, \ldots, r$ and $j=1, \ldots, k$ can be omitted. If there are a handle and a tooth that have 2 or more vertices in common, then the left-hand side of (8.5) has some coefficients equal to 2 , since edges that are contained in both the handle and the tooth are counted twice. Unfortunately, the intersection patching theorem does not provide a short proof for 'facetness' in this case.

A last example of intersection patching concerns the knapsack polytope, which is defined as follows. Given a vector $a \in \mathbb{Z}_{+}^{n}$ and an integer $b \geq \max \left\{a_{i} \mid i=\right.$ $1, \ldots, n\}$, the set $X \subseteq\{0,1\}^{n}$ of feasible points is defined by

$$
X:=\left\{x \in\{0,1\}^{n} \mid \sum_{i=1}^{n_{b}} a_{i} x_{i} \leq b\right\}
$$

and $K S(X):=\operatorname{conv}(X)$, the convex hull of all binary vectors contained in halfspace $H=\left\{x \in \mathbb{R}^{n} \mid a^{T} x \leq b\right\}$. Notice that the index set is simply given by $I=\{1, \ldots, n\}$. Let us first give some elementary properties of the knapsack polytope. A subset $J \subseteq I$ is called an independent set if its characteristic vector belongs to $X$; otherwise $J$ is a dependent set. Obviously, the zero vector belongs to $K S(X)$, and since $b \geq \max \left\{a_{i} \mid i=1, \ldots, n\right\}$ also all unit vectors are contained in this polytope. We already observed that this implies that $x_{i} \geq 0$ defines a facet of $K S(X)$ for all $i \in I$. In addition, $x_{i} \leq 1$ 
defines a facet if $\{i, j\}$ is an independent set for all $j \in I \backslash\{i\}$, as can be easily verified. Notice that if $J \subseteq I$ is a dependent set, then

$$
\sum_{i \in J} x_{i} \leq|J|-1
$$

is a valid inequality for $K S(X)$. A dependent set is minimal if all of its proper subsets are independent. If a dependent set $J \subseteq I$ is not minimal, then (8.6) is implied by

$$
\sum_{i \in J^{\prime}} x_{i} \leq\left|J^{\prime}\right|-1
$$

and $x_{i} \leq 1$ for all $i \in J \backslash J^{\prime}$, where $J^{\prime} \subseteq J$ is a minimal dependent set. However, sometimes inequalities (8.6) define facets of $K S(X)$. To illustrate patching for the knapsack polytope consider

$$
X:=\left\{x \in\{0,1\}^{5} \mid 10 x_{1}+7 x_{2}+7 x_{3}+4 x_{4}+4 x_{5} \leq 21\right\}
$$

Since $J_{1}=\{1,2,3\}$ and $J_{2}=\{2,3,4,5\}$ are dependent sets, the inequalities $x\left(J_{1}\right) \leq 2$ and $x\left(J_{2}\right) \leq 3$ are valid for $P(X)$, and one easily verifies that these inequalities are even facet defining. Next, denote the valid inequality $x(I) \leq 3$ by $\pi^{T} x \leq \pi_{0}$. Then $\operatorname{load}\left(\pi, J_{1}\right)=2$ and $\operatorname{res}\left(\pi, J_{1}\right)=1$, since $R\left(J_{1}\right)$ contains the incidence vector of $\{4\}$. Similarly, $\operatorname{load}\left(\pi, J_{2}\right)=3$, and hence the residual is 0 . The intersection graph of $J_{1}$ and $J_{2}$ with respect to $\pi^{T} x \leq \pi_{0}$ is simply $K_{2}$, and hence all conditions of Theorem 8.3 are met. So we conclude that $x(I) \leq 3$ defines a facet of the kmapsack polytope $K S(X)$.

\subsection{Disjoint patching}

The intersection patching procedure described in the previous section requires that the intersection graph of $I_{1}, \ldots, I_{k} \subseteq I$ with respect to a given valid inequality for $P(X)$ is connected. The disjoint patching procedure requires exactly the opposite: $I_{1}, \ldots, I_{k}$ must form a partition of $I$, so that the intersection graph consists of isolated vertices. Since all definitions needed are already given before we might as well formulate the disjoint patching theorem right now. 


\section{Theorem 8.4 (Disjoint patching)}

Let $a^{T} x \leq a_{0}$ be a valid inequality for $P(X)$ with $a_{0}>0$, and let $I_{1}, \ldots, I_{k} \subseteq I$ be such that

(i) $\bigcup_{j=1}^{k} I_{j}=I$

(ii) $I_{i} \cap I_{j}=\varnothing$ for all $1 \leq i<j \leq k$,

(iii) $\sum_{i \in I_{j}} a_{i} x_{i} \leq a_{0}$ defines a facet of $P\left(L\left(I_{j}\right)\right)$ for all $j=1, \ldots, k$.

Then the inequality $a^{T} x \leq a_{0}$ defines a facet of $P(X)$.

Proof First notice that, since $X$ contains the zero vector and all unit vectors, $L_{(}\left(I_{j}\right)$ contains the zero vector and $\left|I_{j}\right|$ unit vectors, and hence $\operatorname{dim}\left(P\left(L\left(I_{j}\right)\right)\right)=$ $\left|I_{j}\right|$ for all $j=1, \ldots, k$. According to condition (iii) there exists a set $M_{j} \subseteq$ $L\left(I_{j}\right)$ of $\left|I_{j}\right|$ affinely independent points satisfying

$$
\sum_{i \in I_{j}} a_{i} x_{i}=a_{0} \quad \forall x \in M_{j} \quad(j=1, \ldots, k)
$$

Since $a_{0}>0$ none of the sets $M_{j}$ contains the zero vector, so each set consists of linearly independent vectors. Moreover, since $I_{i} \cap I_{j}=\varnothing$ for all $1 \leq i<j \leq k$ the union $M:=M_{1} \cup \ldots \cup M_{k}$ is also linearly independent and $|M|=n$. Since all elements of $M$ belong to the face induced by $a^{T} x \leq a_{0}$ it follows that the latter inequality defines a facet of $P(X)$.

Again, we use the independent set poly tope to illustrate the disjoint patching theorem. Consider the graph $G=(V, E)$ shown in Figure 8.4. If we set $I_{1}:=$ $\{1, \ldots, 5\}$ and $I_{2}:=\{6,7\}$, then there is no independent set in $G$ containing: vertices from both $I_{1}$ and $I_{2}$, hence the inequality

$$
x\left(I_{1}\right)+2 x\left(I_{2}\right) \leq 2
$$

is valid for $P_{15}(G)$. Moreover, $I_{1}$ and $I_{2}$ form a partition of the vertex set $V$. and if $a^{T} x \leq a_{0}$ denotes (8.7) then the inequalities

$$
\sum_{i \in I_{1}} a_{i} x_{i} \leq a_{0}, \quad \sum_{i \in I_{2}} a_{i} x_{i} \leq a_{0}
$$




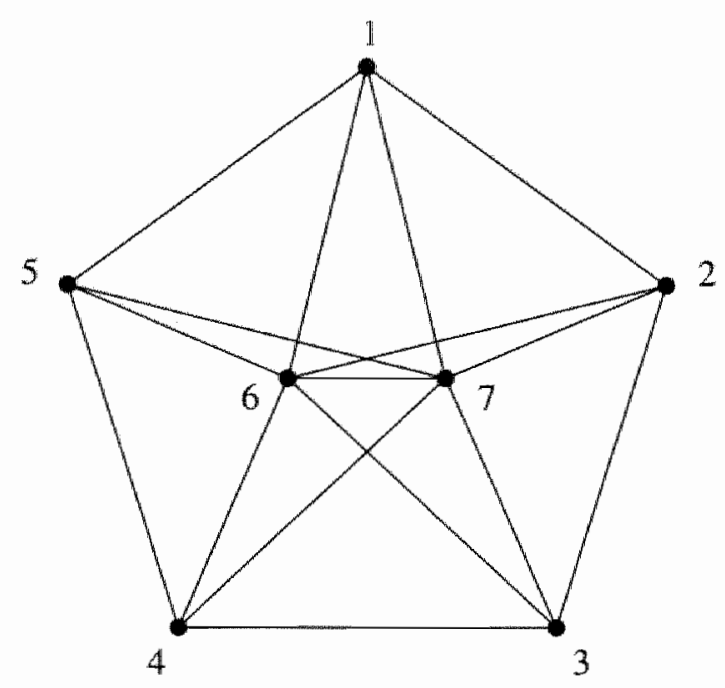

Figure 8.4: An instance of the independent set problem.

are a facet defining odd hole inequality and a clique inequality, respectively. Hence, all three conditions of Theorem 8.4 are met, and we conclude that (8.7) defines a facet of $P_{I S}(G)$. In facet, the 7 nonnegativity constraints, the 5 maximal clique constraints, and inequality (8.7) form a complete description of $P_{I S}(G)$.

An immediate consequence of Theorem 8.4 for the independent set polytope is the following corollary.

Corollary 8.5 Let $G=(V, E)$ be a graph, and let $V_{1}, \ldots, V_{k}$ be a partition of $V$, such that $\left|E\left(V_{i}, V_{j}\right)\right|=\left|V_{i}\right| \cdot\left|V_{j}\right|$ for all $1 \leq i<j \leq k$. Further, let $a_{i}^{T} x \leq b_{i}(i=1, \ldots, k)$ be a facet defining inequality for $P_{I S}\left(V_{i}, E\left(V_{i}\right)\right)$, then the inequality"

$$
\sum_{i=1}^{k} \frac{1}{b_{i}} \cdot\left(a_{i}^{T} x\right) \leq 1
$$

is valid and facet defining for $\operatorname{Pr}(G)$. 


\section{Bibliography}

[1] Adams, P., Lee, Y., Sherali, H.D., A simultaneous lifting strategy for identifying new classes of facets for the boolean quadratic polytope, Operations Research Letters 17 (1995), pp. 19-26.

[2] Ahuja, R.K., Magnanti, T.L., Orlin, J.B., Network flows, 1993, Prentice Hall, Englewood Cliffs, New Jersey.

[3] Balas, E., Zemel, E., Graph substitution and set packing polytopes, Networks 7 (1977), pp. 267-284.

[4] Balas, E., Fischetti, M., A lifting procedure for the asymmetric traveling salesman polytope and a large new class of facets, Mathematical Programming 58 (1993), pp. 325-352.

[5] Barahona, F., Grötschel, M., Mahjoub, A.R., Facets of the bipartite subgraph polytope, Mathematics of Operations Research 10 (1985), pp. 340358.

[6] Barahona, F., Mahjoub, A.R., On the cut polytope, Mathematical Programming 36 (1986), pp. 157-173.

[7] Bhasker, J., Samad, T., The clique partitioning problem, Computers and mathematics with applications 22 (1991), pp. 1-11.

[8] Bondy, J.A., Murty, U.S.R., Graph theory with applications, 1977, Macmillan Press Ltd., London.

[9] Borndörfer, R., Ferreira, C.E., Martin, A., Matrix decomposition by branch-and-cut, Preprint SC-97-14 (1997), Konrad-Zuse-Zentrum für Informationstechnik Berlin. 
[10] Borndörfer, R., Ferreira, C.E., Martin, A., Decomposing matrices into blocks, Preprint SC-97-15 (1997), Konrad-Zuse-Zentrum für Informationstechnik Berlin.

[11] Caprara, A., Fischetti, M., $\left\{0, \frac{1}{2}\right\}$-Chvátal-Gomory cuts, Mathematical Programming 74 (1996), pp. 221-235.

[12] Carraghan, R., Pardalos, P.M., An exact algorithm for the maximum clique problem, Operations Research Letters 9 (1990), pp. 375-382.

[13] Chopra, S., Rao, M.R., On the multiway cut polyhedron, Networks 21 (1991), pp. 51-89.

[14] Chopra, S., Rao, M.R., The partition problem, Mathematical Programming 59 (1993), pp. 87-115.

[15] Chopra, S., Rao, M.R., Facets of the $k$-partition polytope, Discrete Applied Mathematics 61 (1995), pp. 27-48.

[16] Chvátal, V., Edmonds polytopes and a hierarchy of combinatorial problems, Discrete Mathematics 4 (1973), pp. 305-337.

[17] Chvátal, V., Edmonds polytopes and weakly hamiltonian graphs, Mathematical Programming 5 (1973), pp. 29-40.

[18] Chvátal, V., On certain polytopes associated with graphs, Journal of Combinatorial Theory 18 (1975), pp. 138-154.

[19] Chvátal, V., Linear programming, 1983, W.H. Freeman, New York.

[20] Conforti, M., Rao, M.R., Sassano, A., The equipartition polytope I: Formulations, dimension and basic facets, Mathematical Programming 49 (1990), pp. 4970 .

[21] Conforti, M., Rao, M.R., Sassano, A., The equipartition polytope II: Valid inequalities and facets, Mathematical Programming 49 (1990), pp. 71-90.

[22] Crama, Y., Oosten, M., Models for machine-part grouping in cellular manufacturing, International Journal of Production Research 34 (1996), pp. $1693-1713$.

[23] Deza, M., Laurent, M., Facets for the cut cone I, Mathematical Programming 56 (1992), pp. 121-160. 
[24] Deza, M., Laurent, M., Facets for the cut cone II: Clique-web inequalities, Mathematical Programming 56 (1992), pp. 161-188.

[25] Deza, M., Grötschel, M., Laurent, M., Clique-web facets for multicut polytopes, Mathematics of Operations Research 17 (1992), pp. 981-1000.

[26] Dijkhuizen, G., Faigle, U., A cutting plane approach to the edge-weighted maximal clique problem, European Journal of Operational Research 69 (1993), pp. 121-130.

[27] Faigle, U., Schrader, R., Suletzki, R., A cutting plane algorithm for optimal graph partitioning, Methods of Operations Research 57 (1987), pp. 109-116.

[28] Fischetti, M., Clique tree inequalities define facets of the asymmetric traveling salesman polytope, Discrete Applied Mathematics 56 (1995), pp. $9-18$.

[29] Ferreira, C.E., Martin, A., Souza, C.C. de, Weismantel, R., Wolsey, L.A., Formulations and valid inequalities for the node capacitated graph partitioning problem, Mathematical Programming 74 (1996), pp. 247-266.

[30] Fukuda, K., CDD Reference manual, ETH Zentrum, Zürich, Switzerland. Available by anonymous ftp at:

ftp://ifor13.ethz.ch/pub/fukuda/cdd/cdd-060.tar.gz

[31] Garey, M.R., Johnson, D.S., Computers and intractability: a guide to the theory of NP-completeness, 1979, W.H. Freeman, New York.

[32] Gerards, A.M.H., Testing the odd bicycle wheel inequalities for the bipartite subgraph polytope, Mathematics of Operations Research 10 (1985), pp. $359-360$.

[33] Goemans, M.X., Worst-case comparison of valid inequalities for the TSP, Mathematical Programming 69 (1995), pp. 335-349.

[34] Grötschel, M., Padberg, M.W., On the symmetric travelling salesman problem I: Inequalities, Mathematical Programming 16 (1979), pp. 265280 .

[35] Grötschel, M., Padberg, M.W., On the symmetric travelling salesman problem II: Lifting theorems and facets, Mathematical Programming 16 (1979), pp. 281-302. 
[36] Grötschel, M., Pulleyblank, W.R., Weakly bipartite graphs and the maxcut problem, Operations Research Letters 1 (1981), pp. 23-27.

[37] Grötschel, M., Pulleyblank, W.R., Clique tree inequalities and the symmetric travelling salesman problem, Mathematics of Operations Research 11. (1986), pp. 537-569.

[38] Grötschel, M., Wakabayashi, Y., A cutting plane algorithm for a clustering problem, Mathematical Programming 45 (1989), pp. 59-96.

[39] Grötschel, M., Wakabayashi, Y., Facets of the clique partitioning polytope, Mathematical Programming 47 (1990), pp. 367-387.

[40] Grötschel, M., Wakabayashi, Y., Composition of facets of the clique partitioning polytope, (In: Topics in combinatorics and graph theory, R. Bodendiek and R. Henn, eds.), 1990, Physica-Verlag Heidelberg, pp. 271284.

[41] Gupta, A., Fast and effective algorithms for graph partitionings and sparse-matrix ordering, IBM Journal of Research and Development 41. (1997), pp. 171-184.

[42] Hadley, S.W., Finding part-machine families using graph partitioning techniques, International Journal of Production Research 34 (1996), pp. $1821-1839$.

[43] Hansen, P., Jaumard, B., Chuster analysis and mathematical programming, Mathematical Programming 79 (1997), pp. 191-215.

[44] Hartigan, J.A., Clustering algorithms, 1975, John Wiley \& Sons Ltd., New York.

[45] Hyer, N.L., Wemmerlöv, U., Group technology in the US manufacturing inclustry: a survey of current practices, International Journal of Production Research 29 (1989), pp. 287-304.

[46] Johnson, E.L., Mehrotra, A., Nemhauser, G.L., Min-cut clnstering, Mathematical Programming 62 (1993), pp. 133-151.

[47] Kumar, K.R., Kusiak, A., Vanelli, A., Grouping parts and components in flexible manufacturing systems, European Journal of Operational Research 24 (1986), pp. 387-397. 
[48] Laurent, M., Souza, C.C. de, Some new classes of facets for the equicut polytope, Discrete Applied Mathematics 62 (1995), pp. 167-191.

[49] Lawler, E.L., Lenstra, J.K., Rinnooy Kan, A.H.G., Shmoys, D.B., The traveling salesman problem: a guided tour of combinatorial optimization, 1985, John Wiley \& Sons Ltd., New York.

[50] Lee, J., Morris, W.D., Geometric comparison of combinatorial polytopes, Discrete Applied Mathematics 55 (1994), pp. 163-182.

[51] Lint, J.H. van, Wilson, R.M., A course in combinatorics, 1992, Cambridge University Press, Cambridge, United Kingdom.

[52] Macambira, E.M., Souza, C.C. de, The edge-weighted clique problem: valid inequalities, facets and polyhedral computations, Relatório Técnico IC-97-14, Universidade Estadual do Ceará, Brazil.

[53] Marcotorchino, F., Michaud, P., Heuristic approach of the similarity aggregation problem, Methods of Operations Research 43 (1981), pp. 395404.

[54] Mirkin, B., Mathematical classification and clustering, 1996, Kluwer Academic Publishers, Dordrecht, The Netherlands.

[55] Müller, R., Schulz, A.S., The interval order polytope of a digraph, Proceedings of the 4th IPCO Conference (E. Balas and J. Clausen eds.), Lecture Notes in Computer Science 920, 1995, Springer-Verlag, Berlin, pp. $50-64$.

[56] Müller, R., On the partial order polytope of a digraph, Mathematical Programming 73 (1996), pp. 31-49.

[57] Naddef, D., Rinaldi, G., The crown inequalities for the symmetric traveling salesman polytope, Mathematics of Operations Research 17 (1.992), pp. 308-326.

[58] Nemhauser, G.L., Wolsey, L.A., Integer and combinatorial optimization, 1988, John Wiley \& Sons Ltd., New York.

[59] Oosten, M., A polyhedral approach to grouping problems, 1996, Ph.D. thesis, Maastricht University, The Netherlands. 
[60] Padberg, M.W., On the facial structure of set packing polyhedra, Mathematical Programming 5 (1973), pp. 199-215.

[61] Padberg, M.W., The boolean quadratic polytope: some characteristics, facets and relatives, Mathematical Programming 45 (1989), pp. 139-172.

[62] Park, K., Lee, K., Park, S., An extended formulation for the edgeweighted maximal clique problem, European Journal of Operational Research 95 (1996), pp. 671-682.

[63] Schrijver, A., The theory of linear and integer programming, 1986, John Wiley \& Sons Ltd., New York.

[64] Wakabayashi, Y., Aggregation of binary relations: algorithmic and polyhedral investigations, 1986, Ph.D. thesis, Universität Augsburg, Germany.

[65] Wolsey, L.A., Faces for a linear inequality in 0-1 variables, Mathematical Programming 8 (1975), pp. 165-178.

[66] Wolsey, L.A., Further facet generating procedures for vertex packing polytopes, Mathematical Programming 11 (1976), pp. 158-163.

[67] Zemel, E., Lifting facets of zero-one polytopes, Mathematical Programming 15 (1978), pp. 268-277. 


\section{Summary}

The title of this thesis, Polyhedral clustering, is twofold. On the one hand it refers to the polyhedral approach to clustering problems, and on the other hand it refers to a cluster of related polyhedra. Both aspects are treated in this thesis.

The polyhedral approach is applied to two different clustering problems. First, the clique partitioning problem is considered, which can be formulated as follows. Given is a graph $G=(V, E)$ with weights, both positive and negative, for all edges of the graph. Find a partition of $V$, such that each element of the partition induces a clique in $G$ and the sum of the weights on the edges withim the elements of the partition is maximized. The clique partitioning polytope $P(G)$ is the convex hull of all edge sets of clique partitions of $G$. This gives an implicit description of this polytope. Polyhedral combinatiorics is the art of finding an explicit description. Several techniques from this branch of mathematics are used to reveal the structure of the clique partitioning polytope, such as establishing ties with other polyhedra whose structure is already (partially) known, identifying new classes of facets, generalizing individual facets to classes, and lifting facets of the clique partitioning poly tope of subgraphs to facets of the polytope corresponding to the original graph. Next to these techniques patching is used, a new technique for constructing facets from facets. Patching combines the structure of a number of facets to obtain a new facet. One could say that the known structure of a polyhedron is used to reveal part of the unknown structure. Another aspect of the clique partitioning problem that is considered is separation, that is: given a graph $G=(V, E)$ and a point $x \in[0,1]^{|E|}$, find a hyperplane in $\mathbb{R}^{|E|}$ separating $P(G)$ and $x$, or prove that no such hyperplane exists. For some classes of facets the separation problem is NP-hard, whereas for other classes the separation problem is polynomially solvable. 
The second clustering problem considered in this thesis is the graph decomposition problem, which can be formulated as follows. Given is a graph $G=(V, E)$ and an integer $c \geq 1$. Find a subset $S \subseteq V$ of minimal size, such that the graph obtained from $G$ by deleting all vertices in $S$ and all edges incident to $S$ has no component containing more than $c$ vertices. For $c=1$ this problem is equivalent to finding a maximal set of independent vertices in $G$, and therefore the graph decomposition problem is NP-hard in general. The structure of the corresponding polytope $P(G, c)$ is revealed with help of the same techniques as used for the clique partitioning polytope, in particular lifting and establishing ties with other polyhedra. Furthermore, it is examined how useful the known structure of this polytope is to solve instances of this clustering problem.

The second aspect of polyhedral clustering, namely clusters of related polyhedra, is the subject of research in the first and the last chapter of this thesis. The clique partitioning polytope belongs to a family of polyhedra, which also contains the clique polytope, the (multi)cut polytope, and the bipartite subgraph polytope. A more extended overview of this family of polyhedra is given in Figure 1.3. Another cluster is formed by the set of $\{0,1\}$-polyhedra for which patching is a useful technique to investigate the structure. Different variations of patching for these kinds of polytopes are described in the last chapter. 


\section{Samenvatting}

De titel van dit proefschrift, Polyhedrale clustering, is tweeledig. Enerzijds verwijst zij naar de polyhedrale benadering van clusteringsproblemen, anderzijds verwijst zij naar een cluster van gerelateerde polyhedra. Beide aspecten komen in dit proefschrift aan bod.

De polyhedrale benadering wordt toegepast op twee verschillende clusteringsproblemen. Als eerste wordt het kliek-partitie probleem beschouwd, dat, als volgt geformuleerd kan worden. Gegeven is een graaf $G=(V, E)$ met gewichten, zowel positief als negatief, voor alle kanten. Gevraagd wordt een partitie van $V$ zodanig dat elk element van de partitie een kliek in $G$ induceert, en de som van de gewichten op de kanten binnen de elementen van de partitie maximaal is. Het kliek-partitie polytoop $P(G)$ is de convex omhullende van alle kanten-verzamelingen van kliek-partities van $G$. Dit geeft een impliciete beschrijving van dit polytoop. Polyhedrale combinatoriek is de kunst van het vinden van een expliciete beschrijving. Er worden diverse technieken uit deze tak van de wiskunde gebruikt om de structuur van het kliek-partitie polytoop bloot te leggen, zoals het leggen van verbanden met andere polyhedra waarvan de structuur al (gedeeltelijk) bekend is, het identificeren van nieuwe klassen van facetten, het generaliseren van individuele facetten tot uitgebreide klassen, en het liften van facetten van het kliek-partitie polytoop van deelgrafen tot facetten van het polytoop dat correspondeert met de oorspronkelijke graaf. Daarnaast wordt er gebruikt gemaakt van patching, een nieuwe techniek voor het construeren van facetten. Bij patching wordt de structuur van meerdere facetten gecombineerd tot een nieuw facet. Men zou kunnen zeggen dat de bekende structuur van een polyhedron gebruikt wordt om een deel van de onbekende structuur bloot te leggen. Een ander aspect van het kliek-partitie probleem dat belicht wordt is separatie, dat wil zeggen: gegeven een graaf $G=(V, E)$ en een punt $x \in[0,1]^{|E|}$, vind een hypervlak in $\mathbb{R}^{|E|}$ die $P(G)$ en 
$x$ separeert, of bewijs dat zo'n hypervlak niet bestaat. Voor sommige klassen van facetten is het separatieprobleem NP-lastig, terwijl het voor andere klassen polynomiaal oplosbaar is.

Het tweede clusteringsprobleem dat in dit proefschrift beschouwd wordt is het graaf-decompositie probleem, dat als volgt geformuleerd kan worden. Gegeven is een graaf $G=(V, E)$ en een geheel getal $c \geq 1$, gevraagd wordt een deelverzameling $S \subseteq V$ van minimale grootte, zodanig dat de graaf die uit $G$ ontstaat door alle punten uit $S$ en alle kanten incident met $S$ te verwijderen geen componenten heeft die meer dan $c$ punten bevatten. Voor $c=1$ is dit probleem equivalent met het vinden van een maximale onafhankelijke verzameling punten in $G$, en bijgevolg is het graaf-decompositie probleem in het algemeen NP-lastig. De structuur van het bijbehorende polytoop $P(G, c)$ wordt bloot gelegd met behulp van dezelfde technieken die zijn gebruikt voor het kliekpartitie polytoop, met name lifting en het leggen van verbanden met andere polyhedra. Daarnaast wordt nagegaan hoe bruikbaar de bekende structuur van dit polytoop is om instanties van dit clusteringsprobleem op te lossen.

Het tweede aspect van polyhedrale clustering, namelijk verzamelingen van gerelateerde polyhedra, komt aan bod in het eerste en het laatste hoofdstuk van dit proefschrift. Het kliek-partitie polytoop behoort tot een familie van polyhedra, waartoe ook het kliekpolytoop, het (meervoudige) snedenpolytoop, en het bipartiete deelgraaf-polytoop behoren. Een uitgebreider overzicht van deze familie van polyhedra is gegeven in Figuur 1.3. Een ander cluster wordt gevormd door de verzameling van $\{0,1\}$-polytopen waarvoor patching een geschikte techniek is om de stuctuur te onderzoeken. Verschillende varianten van patching voor deze polytopen worden beschreven in het laatste hoofdstuk. 


\section{Curriculum vitae}

Jeroen Rutten was born on February 19, 1970 in Breda, the Netherlands. From 1982 to 1988 he attended secondary education at the Rijksscholengemeenschap Koning Willem II in Tilburg. Subsequently he studied mathematics at the Eindhoven University of Technology. During his study he worked for almost two years as a teaching assistant, first at the department of Computer Science on spline approximation, later at the department of Mathematics on linear algebra. In 1994 he graduated in combinatorial optimization and operations research under the guidance of prof.dr. J.K. Lenstra. He wrote his Master's thesis on the application of polyhedral combinatorics to clustering problems, which gave the first impuls to this thesis. In March 1994 he started his work as a research assistant at the department of Mathematics of the Maastricht University. Currently he is working for ASM Lithography in Veldhoven. 


\section{Index}

2-chorded cycle inequality, 23,40

2-chorded path inequality, 40

2-chorded wheel inequality, 40

2-partition inequality, 22, 39

affinely independent, 9

algorithm

branch-and-cut, 17, 93, 112, 114, 144,146

cutting plane, 94

exact, 149

heuristic, 8

recursive, 148

antiweb, 41

backbone, 5

basic inequality, 22

Bell number, 100

bicycle wheel inequality, 42

bordered block diagonal form, 125

center, 58

characteristic vector, 14

chord, 13

Chvátal-Gomory cut, 118

circular reference, see reference, circular

classification, 6

claw inequality, 42

clique, 13

clique cover, 8,47

clique cover number, 48 clique partitioning, 8, 14, 21

clique tree inequality, 163

clique-web inequality, 41

closure, 118

clustering, 1

column generation, 18

comb inequality, 162

composed 2-partition inequality, 76

cone, 10

$$
\text { cut, } 16
$$

connectivity

edge, 12

vertex, 12

constraint

capacity, 127

clique, 112

connectivity, 127

degree, 26

integrality, 127

nonnegativity, 22

subtour elimination, 112

upper bound, 22,58

convex, 8

combination, 8

hull, 8

cover

clique, 8

covering, 62

cut, 16

cycle, 12 
degree, 13

degree constraint, 26

dependent set, 165

description

complete, 84

partial, 84

dimension, 11

full, 11

disconnecting graphs, 125

disjoint patching, 39, 61, 70, 72, 74, 167

dissimilarity, 7

edge, 11

exception, 4

extreme point, 11

face, 11

proper, 11

facet, 11

homogeneous, 121

trivial, 22

flexible manufacturing system, 4

Gaussian elimination, 45

gnu, 2

graph

complete, 12

comnected, 12

intersection, 31, 159

triangle-free, 13

graph partitioning, 126

group technology, 126

halfspace, 9

handle, 163

hole, 160

hull

affine, 10

convex, 8 hyperplane, 9

incidence vector, 14

independent

affinely, 9

linearly, 9

independent set, 160,165

inequality

2-chorded cycle, 23,40

2-chorded path, 40

2-chorded wheel, 40

2-partition, 22, 39

4-cycle, 143

arrow, 142

basic, 22

bicycle wheel, 42

block invariant, 134

circuit, 137

claw, 42

clique, 139,160

clique tree, 163

clique-web, 41

comb, 162

composed 2-partition, 76

general 2-partition, 34,37

generalized 2-chorded cycle, 51 , 53

generalized 2-chorded path, 57

generalized 2-chorded wheel, 57

generalized cover, 138

hole, 160

$K_{1,2}, 22$

lifted claw, 60

lifted stable set, 60

stable set, 48

star, 58,139

triangle, 22,127

valid, 11

wheel, 41 
inheritance

of facets, 135

of valid inequalities, 134

intersection graph, 31, 159

intersection patching, $39,61,65,159$

lifted claw inequality, 60

lifted stable set inequality, 60

lifting

sequential, 58,157

simultaneous, 157

zero, 23,24

linearly independent, 9

load, 30,158

matching, 13

measure for strength

distance, 98

extreme point, 95

ratio, 96

Miplib, 144

multicut, 15

Netlib, 144

network.

local area, 5

wide area, 5

partition, 3

patching, $31,39,61,157$

basic principle, 63

disjoint, $39,61,70,72,167$

disjoint $(S, T), 74$

intersection, 39, 61, 65, 159

star, 69

path, 12

pattern recognition, 5

Periodic Chart, 7

point

extreme, 11 polyhedron, 10

blocking, 97

polytope, 10

bipartite subgraph, 16

capacitated clique partitioning, 17,133

clique, 17,133

clique partitioning, 14

cut, 16

equicut, 16

equipartition, 16

independent set, 160

knapsack, 165

multicut, 16

traveling salesman, 162

zero-identical, 97

production cell, 4, 126

projected vector, 24

proper face, $\mathbb{1 1}$

rank, 118

reference

circular, see circular reference

resemblance, 3

residual, 30,158

separation, 111

$\left\{0, \frac{1}{2}\right\}$-Chvátal-Gomory cut, 118

2-chorded cycle inequality, 119

2-partition inequality, 113

bicycle wheel inequality, 121

claw inequality, 117

composed 2-partition inequality, 123

wheel inequality, 119

similarity, 7

stability number, 47

stable set inequality, 48 
star inequality, 58

Stirling number, 99

strength, 96, 98

subgraph, 13

support, 14

switching, 120

tooth, 163

triangle inequality, 22

truncated vector, 24

ubiquitous, 1

valid inequality, 11

vector

characteristic, 14

incidence, 14

projected, 24

truncated, 24

vertex, 11

void, 4

volume of polyhedra, 94

walk, 12

web, 41

whee inequality, 41

wild cats, 3

zero lifting, 23,24 . 

star inequality, 58

Stirling number, 99

strength, 96, 98

subgraph, 13

support, 14

switching, 120

tooth, 163

triangle inequality, 22

truncated vector, 24

ubiquitous, 1

valid inequality, 11

vector

characteristic, 14

incidence, 14

projected, 24

truncated, 24

vertex, 11

void, 4

volume of polyhedra, 94

walk, 12

web, 41

wheel inequality, 41

wild cats, 3

zero lifting, 23,24 